\title{
Investigating human neocortical architecture in 3D
}

\author{
Citation for published version (APA):
}

Hildebrand, S. (2021). Investigating human neocortical architecture in 3D: new approaches for clearing, labelling and imaging large samples. [Doctoral Thesis, Maastricht University]. Maastricht University. https://doi.org/10.26481/dis.20211214sh

Document status and date:

Published: 01/01/2021

DOI:

10.26481/dis.20211214sh

Document Version:

Publisher's PDF, also known as Version of record

\section{Please check the document version of this publication:}

- A submitted manuscript is the version of the article upon submission and before peer-review. There can be important differences between the submitted version and the official published version of record.

People interested in the research are advised to contact the author for the final version of the publication, or visit the DOI to the publisher's website.

- The final author version and the galley proof are versions of the publication after peer review.

- The final published version features the final layout of the paper including the volume, issue and page numbers.

Link to publication

\footnotetext{
General rights rights.

- You may freely distribute the URL identifying the publication in the public portal. please follow below link for the End User Agreement:

www.umlib.nl/taverne-license

Take down policy

If you believe that this document breaches copyright please contact us at:

repository@maastrichtuniversity.nl

providing details and we will investigate your claim.
}

Copyright and moral rights for the publications made accessible in the public portal are retained by the authors and/or other copyright owners and it is a condition of accessing publications that users recognise and abide by the legal requirements associated with these

- Users may download and print one copy of any publication from the public portal for the purpose of private study or research.

- You may not further distribute the material or use it for any profit-making activity or commercial gain

If the publication is distributed under the terms of Article $25 \mathrm{fa}$ of the Dutch Copyright Act, indicated by the "Taverne" license above, 


\title{
Investigating human neocortical
} architecture in 3D:

New approaches for clearing,

labelling and imaging large samples

\author{
Sven Hildebrand
}


(c) Sven Hildebrand, Maastricht 2021

The research presented in this thesis was supported by the Netherlands Organisation for Scientific Research (NWO, VIDI to Alard Roebroeck \#14637).

The research was conducted at Maastricht University.

Cover Sven Hildebrand

Printing Ipskamp Printing - www.ipskampprinting.nl

ISBN 978-94-6423-592-0

DOI https://doi.org/10.26481/dis.20211214sh 


\title{
Investigating human neocortical architecture in 3D:
}

New approaches for clearing, labelling and imaging large samples

\section{Dissertation}

\author{
Approved, after corrections, \\ Prof.dr. Rianne M. Letschert, Rector Magnificus, \\ To be defended in public on \\ Tuesday the $14^{\text {th }}$ of December 2021, at 10:00 hours
}

By

Sven Hildebrand 


\section{Supervisors}

Dr. Alard F. Roebroeck

Prof. Dr. Rainer W. Goebel

Co-Supervisor

Prof. Dr. Ralf A. W. Galuske

Technische Universität Darmstadt

Assessment Committee

Prof. Dr. Peter H. M. de Weerd (Chair)

Prof. Dr. Jos H. H. J. Prickaerts

Dr. Sébastien Foulquier

Prof. Dr. Dr. Svenja Kaspers

Heinrich Heine Universität Düsseldorf

Prof. Dr. Antony Morland

University of York 
"The process leading to the accomplishment of functional localization in the cerebral cortex is such a complicated one, and involves so many side issues, that perfection cannot be attained or even hoped for until the fruits of investigation in a number of departments are thoroughly weighed, sifted and assorted. It is anticipated that the observations set forth in this research will help to establish the value of histological work as an auxiliary force in the final settlement of that functional subdivision of the cerebral cortex at which we aim."

\section{- Alfred Walter Campbell}

"[...] in fact the whole fabric of a man except the red of his blood and the black pigment of hair, are all made up of transparent, colourless tissue. So little suffices to make us visible one to the other. For the most part the fibres of a living creature are no more opaque

than water."

Griffin in "The Invisible Man" by Herbert George Wells

"Bears can ride bicycles, a surprising fact of elusive theoretical significance." 


\section{TABLE OF CONTENTS}

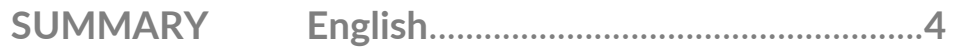

SUMMARY Dutch ........................................................

CHAPTER 1 General Introduction .......................... 15

CHAPTER 2 Scalable Labeling for

Cytoarchitectonic Characterisation

of Large Optically Cleared Human

Neocortex Samples.............................. 69

CHAPTER 3 An investigation of angioarchitecture in the human visual cortex with angioMASH tissue clearing and labelling ................................................125

CHAPTER 4 Taking MASH to the next level: A high-throughput pipeline for the investigation of cytoarchitecture in large human brain slices .................175 
CHAPTER 5 cleared tissue dual Selective Plane Illumination Microscopy (ct-dSPIM) for mesoscopic fast imaging of largescale human tissues 217

CHAPTER 6 hFRUIT: An optimized agent for optical clearing of Dil-stained adult human brain tissue 259

CHAPTER 7 General Discussion 297

IMPACT PARAGRAPH 325

ABBREVIATIONS 344

ACKNOWLEDGEMENTS 347

CURRICULUM VITAE 353

LIST OF PUBLICATIONS 355 


\section{Summary}

Light microscopy has been used extensively for well over a hundred years to study the neuroanatomy of the human brain. The aim of this thesis was to develop methodological aspects of histological tissue processing and microscopy, to overcome some shortcomings of conventional light microscopical investigations of human brain tissue. In particular, a novel histological tool called optical tissue clearing was employed, in order to render large brain samples transparent and thereby enable 3D imaging by allowing light to pass through them. Then, an imaging method called light-sheet fluorescence microscopy was used to quickly image large parts of those transparent samples. By combining these to technologies, large tissue samples of several millimetre thickness can be imaged in their entirety. This in turn reduces the need for computationally intense $3 \mathrm{D}$ reconstructions of thousands of paper-thin histological sections, which is the current standard approach to such large-scale imaging endeavours and requires substantial infrastructure only available in few research centres. Another advantage of imaging tissue slices that are orders of magnitude thicker than standard histological sections is the reduction of shearing and deformation, which is a common problem in very thin histological sections mounted onto glass slides.

In Chapter 2, this novel way to optically clear and label human brain samples for cytoarchitecture is presented. This method is called Multiscale Architectonic Staining of Human cortex (MASH). We show 
the feasibility and scalability of this method by imaging the cytoarchitecture of the occipital neocortex with commercial lightsheet as well two-photon microscopes, with labels of different wavelength. It is further shown that mesoscopic datasets (datasets extending over large field of views of many millimetres to centimetres with resolutions in the single micrometre range) enable the relatively easy location of imaged regions in MRI (Magnetic Resonance Imaging) data on whole occipital lobes. The scope of application of the MASH method is further extended in Chapter 3 by adding a labelling protocol for angioarchitecture to the existing clearing and cytoarchitecture labelling. Using this method in combination with the mesoSPIM microscope, it could be shown that the vascular architecture between human visual areas V1 and V2 is distinct and that orientation profiles change as well as vessel densities change considerably over the cortical depth in a single area. The mesoSPIM is an open-source lightsheet microscope optimized for cleared, mesoscopic samples up to a few centimetres in size. It is hoped that the introduction of this angioMASH technique and similar protocols by other groups, will lead to a revival of the study of angioarchitecture, especially given its new relevance in the context of non-invasive functional imaging modalities such as fMRI (functional MRI) or fNIRS (functional Near-Infrared Spectroscopy). The angioMASH method could also play an important role in investigating human pathologies in which vessel abnormalities are observed, such as vascular dementia or certain forms of epilepsy. To advance the scope of MASH even further, a high-throughput version of this tissue-processing pipeline is shown in Chapter 4, which 
enables the optical clearing of multiple, whole coronal occipital lobe slices. This high-throughput version utilises custom 3D printed laboratory equipment which is easily scalable and hence could facilitate the clearing a substantial volume of the human brain in a matter of days. Samples of this size would be impossible to image with conventional light-sheet microscope set-ups, however, as the excitation light enters the tissue laterally in those systems. Therefore, samples many centimetres in lateral extend need a different kind of light-sheet set-up, in which both orthogonal objectives are placed above the sample, a large sample chamber compatible with the imaging media, and a large stage travel range to cover the entire extend of the tissue slice. A prototype set-up of this kind, the ctdSPIM (cleared-tissue dual view Selective Plane Illumination Microscopy) is introduced in Chapter 5 . The versatility of this system is demonstrated by imaging very large samples of both human brain and prostate at multiple resolutions. The latter is achieved, not by changing objectives as in standard light microscopy, but rather by downsampling the acquired data. This is done both while acquiring it to reduce the data volume and further after the acquisition, to ultimately match the final in-plane resolution with the distance between the image planes - an imaging mode we termed MFS (Mesoscopic Fast Scans). These MFS scans allow for the acquisition of isotropic data at mesoscale resolutions over large volumes without the need of either multi-view deconvolutions or axial scanning of the (gaussian) light-sheet waist. Finally, to add to the repertoire of methods, a specialised clearing method termed hFRUIT is presented 
in Chapter 6. It is a version of the original FRUIT protocol (named after its ingredients fructose, urea and 1-thioglycerol) optimised for human brain tissue labelled with lipophilic tracers. This non-delipidating aqueous clearing protocol is chemically gentle enough to those preserve lipophilic dyes, which is shown in both porcine and human brain tissue, while potent enough to clear human (and porcine) grey matter. hFRUIT could therefore become a powerful tool in the near future to investigate the intrinsic connectivity of the human neocortex, a methodologically challenging field to study because of the limited techniques available to date for selective, local labelling of these intrinsic connections.

Light microscopy has been a crucial tool for the investigation of human (and non-human) brain anatomy. Despite impressive developments in non-invasive brain imaging techniques, the microscopic probing of post mortem brain tissue will continue to play an essential part in neuroanatomy, because of its superior resolution. Yet many features of brain anatomy extend over large distances, which are difficult to capture with conventional microscopy. The mesoscopic features of brain architecture, extending over many millimetres to centimetres, but requiring microscopic resolution, are ideally suited for the novel field of 3D histology which combines optical tissue clearing techniques with 3D microscopy methods such as light-sheet microscopy. This thesis advances the 3D imaging of extensive volumes, by making it scalable to ever larger samples, allowing the imaging of structures of interest such as cortical layers 
observable in e.g. angio- and cytoarchitecture over considerable distances, while at the same time keeping it affordable for standard histology labs to implement. 


\section{Samenvatting}

Lichtmicroscopie wordt al meer dan honderd jaar op grote schaal gebruikt om de anatomie van het menselijk brein te bestuderen. Het doel van het werk in dit proefschrift was om histologische weefselverwerking en -microscopie verder te ontwikkelen, en om enkele tekortkomingen van conventioneel lichtmicroscopisch onderzoek naar menselijk hersenweefsel te ondervangen. Allereerst werd een nieuwe histologische methode, optical tissue clearing (hierna: klaren), gebruikt om grote monsters van het brein transparant te maken. Dit maakte het mogelijk om er licht doorheen te laten schijnen. Vervolgens werd een 3D-beeldvormingstechniek, genaamd light-sheet fluorescence microscopy, gebruikt om met hoge snelheid grote delen van de transparante monsters in beeld te brengen. Door deze twee technieken te combineren, kunnen grote weefselmonsters van enkele millimeters dik in hun geheel in beeld worden gebracht. De huidige standaardbenadering voor dergelijke grootschalige beeldvorming is het maken van duizenden flinterdunne histologische secties, die vervolgens met rekenintensieve technieken in 3D gereconstrueerd worden. De substantiële infrastructuur die hiervoor vereist is, en die slechts in enkele onderzoekscentra beschikbaar is, kan met onze methode geheel uitgespaard worden. Een ander groot voordeel van onze methode, in tegenstelling tot het conventionele gebruik van duizenden op glasplaatjes gemonteerde secties, is de vermindering van afschuiving en vervorming van het weefsel. 
In Hoofdstuk 2 presenteer ik deze nieuwe methode om preparaten van het menselijk brein optisch te klaren en te labelen. Deze methode hebben we Multiscale Architectonic Staining of Human cortex (MASH) genoemd. Ik toon de haalbaarheid en schaalbaarheid van deze methode aan door de celstructuur van de occipitale neocortex in beeld te brengen met commerciële light-sheet- en two-photonmicroscoopen, met labels van verschillende golflengten. Verder is aangetoond dat het gebruik van mesoscopische beelden (beelden die zich uitstrekken over een groot gezichtsveld, van vele millimeters tot centimeters en met resoluties in het bereik van enkele micrometers) van de occipitaalkwab, het relatief gemakkelijk maken de beelden te vergelijken met MRI-beelden (Magnetic Resonance Imaging) van hetzelfde gebied.

Het toepassingsgebied van de MASH-methode wordt in Hoofdstuk 3 verder uitgebreid door een labelingsprotocol voor vasculaire structuur toe te voegen aan de bestaande klaring en celstructuurlabeling. Met behulp van deze methode, en in combinatie met de mesoSPIM-microscoop, werden de menselijke visuele hersengebieden V1 en V2 met elkaar vergeleken. Ik kon aantonen dat de vasculaire architectuur tussen deze twee gebieden verschillend is. Tevens kon ik aantonen dat de oriëntatie van de bloedvaten, evenals de vaatdichtheden, aanzienlijk veranderen wanneer men dieper in de cortex van een enkel gebied kijkt. De mesoSPIM is een open-source light-sheet-microscoop die is geoptimaliseerd voor geklaarde, mesoscopische preparaten die enkele centimeters dik kunnen zijn. 
Het is te hopen dat het gebruik van deze nieuwe techniek, die we angioMASH hebben genoemd, en soortgelijke protocollen door andere onderzoeksgroepen, zal leiden tot een heropleving van de studie van vasculaire architectuur, vooral vanwege de nieuwe relevantie hiervan in de context van niet-invasieve functionele beeldvormingstechnieken zoals fMRI (functionele MRI) of fNIRS (functionele Near Infra-red Spectroscopy). De angioMASH-methode zou ook een belangrijke rol kunnen spelen bij het onderzoeken van menselijke vaat-pathologie, zoals vasculaire dementie of bepaalde vormen van epilepsie.

In Hoofdstuk 4 wordt een high-throughput-versie van deze weefselverwerkingspijplijn getoond, om het toepassingsgebied van MASH verder te vergroten. Deze high-throughput-versie maakt de klaring van meerdere, occipitaalkwab preparaten mogelijk en maakt gebruik van op maat gemaakte, 3D-geprinte, laboratoriumapparatuur. Deze is gemakkelijk en goedkoop opschaalbaar naar meer volume, wat het mogelijk moet maken om een (groot deel van het) menselijk brein in enkele dagen in beeld te brengen. Preparaten van deze omvang zouden onmogelijk kunnen worden afgebeeld met conventionele opstellingen met light-sheets, aangezien het licht in die systemen zijdelings het weefsel binnendringt.

Dergelijke monsters van vele centimeters dikte hebben een andere opstelling van de light-sheet nodig, waarbij beide orthogonale objectieven boven het monster worden geplaatst. Verder is een grote 
monsterkamer nodig, die compatibel is met de beeldvormende vloeistof. Tevens nodig is een groot bereik voor het verplaatsen van het podium waarop het monster staat, om zo het gehele monster te kunnen bereiken. Een prototype van dit soort opstelling, de ct-dSPIM (cleared-tissue dual view Selective Plane Illumination Microscopy) wordt geïntroduceerd in Hoofdstuk 5. De veelzijdigheid van dit systeem wordt gedemonstreerd door beeldvorming, in verschillende resoluties, van zeer grote preparaten van zowel menselijke hersenen als menselijke prostaat. Deze verschillende resoluties worden bereikt, niet door het camera-objectief te veranderen zoals bij standaard lichtmicroscopie, maar door de verkregen gegevens te downsamplen. Dit wordt zowel gedaan tijdens de dataverzameling, om het gegevensvolume te verminderen, als ook na de acquisitie, om de uiteindelijke resolutie in het vlak af te stemmen op de afstand tussen de beeldvlakken - een beeldvormingsmodus die we MFS (Mesoscopic Fast Scans) hebben genoemd. Deze MFS-scans beslaan grote volumes, bestaande uit isotrope gegevens met resoluties op mesoschaal, zonder de noodzaak van deconvolutie of axiaal scannen.

Ten slotte wordt in Hoofdstuk 6, om nog een methode aan het repertoire toe te voegen, een gespecialiseerde methode voor het klaren, genaamd hFRUIT, gepresenteerd. Het is een versie van het originele FRUIT-protocol (genoemd naar de ingrediënten fructose, ureum en 1-thioglycerol), geoptimaliseerd voor menselijk hersenweefsel dat gelabeld is met lipofiele tracers. Dit nietdelipiderende, waterige reinigingsprotocol is chemisch zacht genoeg 
om lipofiele kleurstoffen te behouden, wat wordt aangetoond in zowel varkens- als menselijk hersenweefsel. Tegelijkertijd is het krachtig genoeg om menselijke (en varkens) grijze massa te klaren. hFRUIT zou daarom in de nabije toekomst een krachtig hulpmiddel kunnen worden om de intrinsieke connectiviteit van de menselijke neocortex te onderzoeken - een methodologisch uitdagend veld, vanwege de beperkte technieken die tot nu toe beschikbaar zijn voor selectieve, lokale labeling van deze intrinsieke verbindingen.

Lichtmicroscopie is een essentieel hulpmiddel geweest voor het onderzoek van de menselijke (en niet-menselijke) hersenanatomie. Ondanks indrukwekkende ontwikkelingen in niet-invasieve hersenbeeldvormingstechnieken, zal het microscopisch onderzoek van post-mortem hersenweefsel een essentiële rol blijven spelen in de neuroanatomie, vanwege diens inherent hogere resolutie. Toch strekken veel kenmerken van de hersenanatomie zich uit over grote afstanden, die moeilijk vast te leggen zijn met conventionele microscopie. Deze mesoscopische kenmerken van de hersenarchitectuur, die zich uitstrekken over vele millimeters tot centimeters, maar een microscopische resolutie vereisen, zijn bij uitstek geschikt voor het nieuwe veld van 3D-histologie, dat optische weefselklaringtechnieken combineert met 3D-microscopiemethoden zoals light-sheet microscopie. Dit proefschrift heeft als doel het verbeteren van de $3 \mathrm{D}$-beeldvorming van grote volumes, door deze techniek schaalbaar te maken naar steeds grotere preparaten. Hierdoor wordt het mogelijk om belangrijke structuren, zoals 
corticale lagen, waarneembaar te maken in bijvoorbeeld vaat- en celstructuur in aanzienlijke volumes, terwijl het tegelijkertijd een haalbare techniek blijft voor histologische laboratoria. 
Chapter 1 - General Introduction

ChAPTER 1

\section{General Introduction}




\section{Mapping neocortical architecture}

How can different parts of the human neocortex perform vastly different computational tasks, even though their appearance is remarkably similar? The idea that the neocortex is parcellated into structural units, which underlie and serve its multitude of functions gained traction in the early $20^{\text {th }}$ century $^{1-3}$. This was made possible after specific histochemical stainings for certain cortical components (and ever better optics to inspect them) had been developed in the later part of the $19^{\text {th }}$ century ${ }^{4}$. Two of the most commonly employed features for brain mapping were the organisation of cell bodies

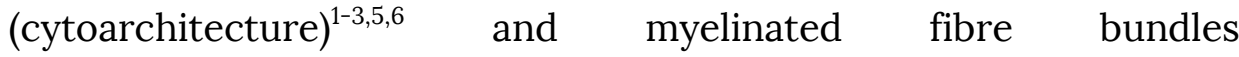
(myeloarchitecture) $)^{7,8}$. The seminal contributions from this time are still regularly used as the reference for many studies. Especially the brain parcellation by Korbinian Brodmann ${ }^{1}$ is frequently employed to this day, although there are many similar maps from different anatomists, which in some cases differ widely in the numbers of delineated areas. Figure 1 shows two classical maps still often used today: Brodmann (middle) and von Economo and Koskinas (right) ${ }^{3}$. On the left side, the map by Bailey and von Bonin ${ }^{9}$ is provided as one of the most striking examples of the variability between anatomists (see "Limitations of classical brain parcellation" below).

While Bailey's and von Bonin's minimalistic map of cortical areas is still useful in depicting the most fundamental differences between cortical regions, finer divisions have garnered more support in the more 
recent past $\mathrm{t}^{10-12}$. Today, most neuroanatomists agree about the division of the cortex in distinct areas, although the question of how many areas exist is still not conclusively answered. An important step towards more reliable brain mapping is the ongoing automatisation for user-independent identification of areal borders ${ }^{5,6,10,11,13-16}$. In addition, orthogonal methodological approaches seem to support a fine parcellation, often in good agreement with structurally identified $\operatorname{maps}^{11,12,17,18}$.
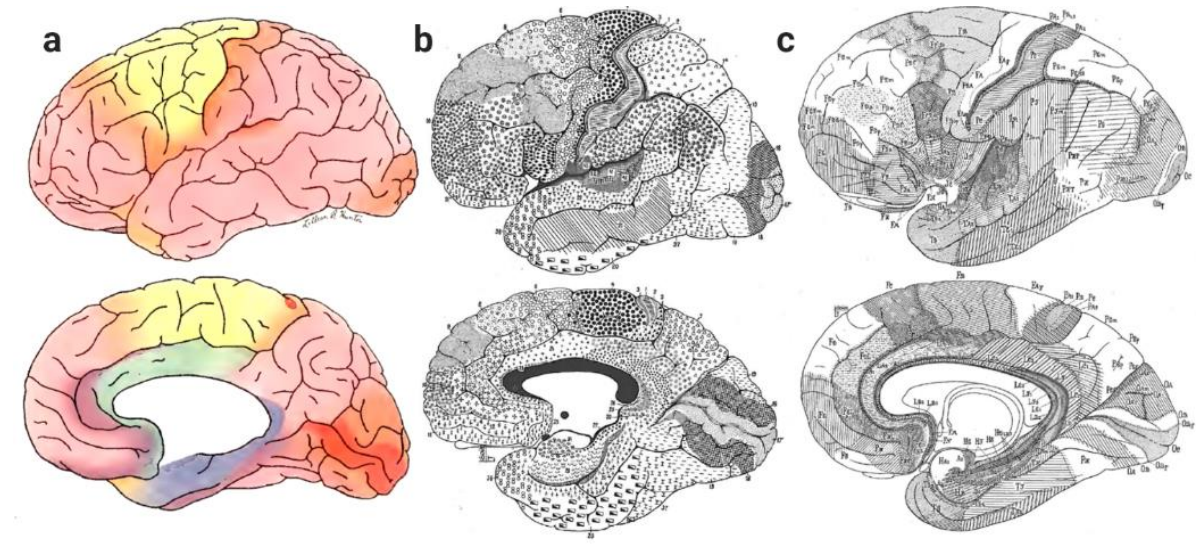

Figure 1: Three historical maps of brain areas as revealed by cytoarchitecture. Top row shows the lateral view, bottom row the medial view of the brain. Note the extreme variability in number and position of areas. a) Map by Bailey and von Bonin. These authors where most critical of the idea of distinctly defined brain areas. However, their map is very similar to the distribution of von Economo's and Koskinas map of main cortex types (see Fig. 4, adapted from Bailey and von Bonin, 1951\%). The maps of Brodmann (b) and von Economo and Koskinas (c) are much more similar, but here considerable differences are apparent as well (both maps adapted from von Economo and Koskinas, 1925³). 
In the next paragraphs, different approaches to study cortical architecture will be introduced, starting with the two most renowned ones: myelo- and cytoarchitecture. Angioarchitecture, another but less known approach for parcellation, is described as well. Lastly, the only method available today for studying cortical connectomics in the human brain at a microscale is presented.

\section{Myeloarchitecture}

One of the first studies of cortical architecture was conducted by Campbell who used both cyto- and myeloarchitecture to map the brain $^{2}$. Nevertheless, arguably the most famous proponents of myeloarchitecture were Cécile and Oskar Vogt, together with their numerous collaborators and disciples.

The Vogts were the first to introduce a general scheme for the myeloarchitectonic organisation of the cortex (Fig. 2a). This general myeloarchitectonic scheme was further divided into larger and smaller subtypes (Fig $2 \mathrm{~b})^{8}$. Though the main criteria for delineating myeloarchitectonic areas are the four different laminar and three different radial types of cortex, the Vogts were very fond of classifying variations into additional subclasses ${ }^{7,8,19}$. This led to a complex typological scheme in which the number and thickness of radial fibre bundles, the ratio of thick and thin fibres in tangential layers, and the overall abundance or scarcity of fibres in general were taken into consideration. Based on this complicated typology, the Vogts and their disciples were able to delineate much smaller parcellations of 
the cortex as compared to Brodmann's cytoarchitectonic map $p^{7,8,19-22}$. This was explicitly remarked upon by Oskar Vogt in a publication following briefly after the first published studies by Brodmann ${ }^{7}$.

Other anatomists such as Adolf Hopf distinguished even more laminar types of myeloarchitecture ${ }^{20,23}$. Not surprisingly, given such a variety between investigators in classification and employed method, the resultant myeloarchitectonic maps show a high variability in the number of areas ${ }^{7}$. These methods ranged from macroscopical investigation of unstained material to simple magnifying glasses to higher magnifications of stained material. Notably though, many areas in all lobes appear reliably in maps by different anatomists. Nevertheless, the observer-dependent nature of brain parcellations proved to be a problem in myeloarchitectonics and similarly for cytoarchitectonics. 

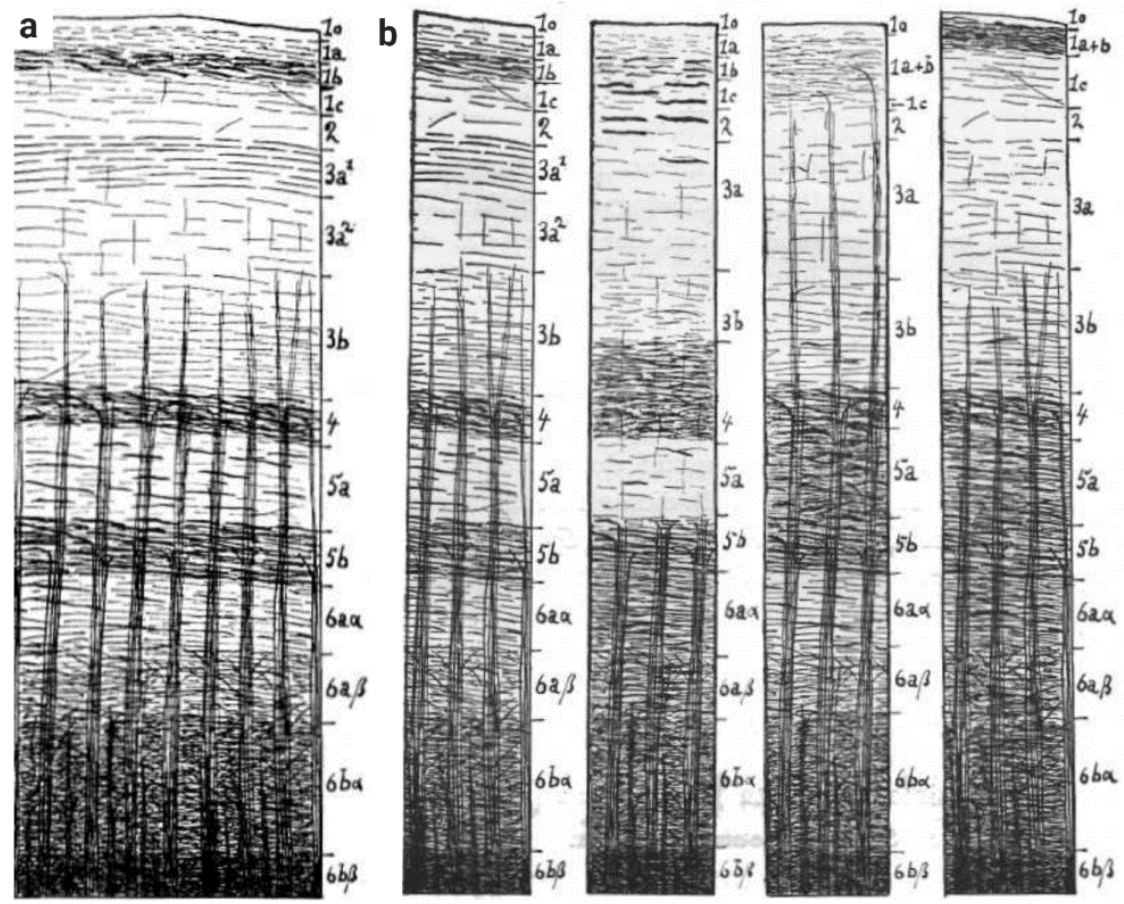

Figure 2: Myeloarchitecture according to the Vogt-Vogt school. a) Basic plan of the myelinated fibres in the six layered neocortex. Main layers are indicated in Arabic numerals (different to the cytoarchitecture layers, which are indicated in Roman numerals). Well visible are the tangential stripes: The Cajal-Retzius stripe in layer $1 \mathrm{~b}$, the Kaes-Bechterew stripe in layer $3 a$ and the two stripes of Baillarger in layer 4 (outer) and layer $5 \mathrm{~b}$ (inner). b) The tangential stripes allow for the differentiation of four main types of cortex. From left to right: The bistriate types shows both stripes of Baillarger, where as in the unistriate type only the outer stripe of Baillarger is visible and in the unitostriate type both are merged into one thick stripe. In the astriate type, a massive abundance of tangential fibres in layers 4-6 masks both stripes. Also visible in these examples are the three main radial types: Euradiate (outer left and right columns), infraradiate (inner left column), and the supraradiate type (inner right column). Adapted from Vogt $1910 b^{8}$ (a) and Nieuwenhuys, $2013^{7}$ (b) respectively. 


\section{Cytoarchitecture}

The invention of the Nissl staining in the late $19^{\text {th }}$ century, a method to selectively label cell nuclei and cytoplasm with several cationic dyes $^{4}$, was the starting point for a flurry of studies on cortical cytoarchitecture from the early $20^{\text {th }}$ century $^{1-3,13,24,25}$. This staining method revealed the basic arrangement of the isocortex (= neocortex) into several layers. Although initially there was some variation in how these layers were counted, today it is generally accepted that the isocortex consists of six layers, although sometimes layers can be (almost) missing or further divided into sublayers (Fig. 3a $)^{26-29}$. Layer I, the molecular or zonal layer, is sparsely populated with cells and contains many afferent corticocortical and thalamocortical fibres (compare Cajal-Retzius stripe in myeloarchitecture). Layers II and IV are the outer and inner granular layers respectively and are followed by the outer (III) and inner (V) pyramidal layer. Although in layer III and $\mathrm{V}$ the pyramidal cells are most obvious, they are also present in other layers, rather counterintuitive to their name. Layer VI, the multiform layer, can contain very different densities of pyramidal and fusiform cell populations (among others), which get less densely populated towards the white matter border. It is for this reason that demarcating a definite white matter-grey matter boundary in Nisslstained preparations can be challenging ${ }^{7}$.

Similar to the Vogt's classification of laminar variations (Fig. 2b), corresponding typologies were introduced for cytoarchitecture as 
well. Figure $3 \mathrm{~b}$ shows the five main types according to von Economo and Koskinas ${ }^{3}$. Type 2-4 show the six layers most clearly and are referred to as homotypical. They are named for the location on which they are predominantly found: The frontal (2), parietal (3), and polar (4) type respectively. Although as figure $3 \mathrm{c}$ shows, these types are not exclusively confined to these brain regions and von Economo stressed that although the polar (4) type can be found both at the anterior and posterior poles, both are markedly different from another. The other two main types are heterotypical, in that they are either lacking in granular cells (agranular (1) type) or that small granular neurons are so abundant that pyramidal layers become difficult to delineate (granulous or koniocortex (5) type). 


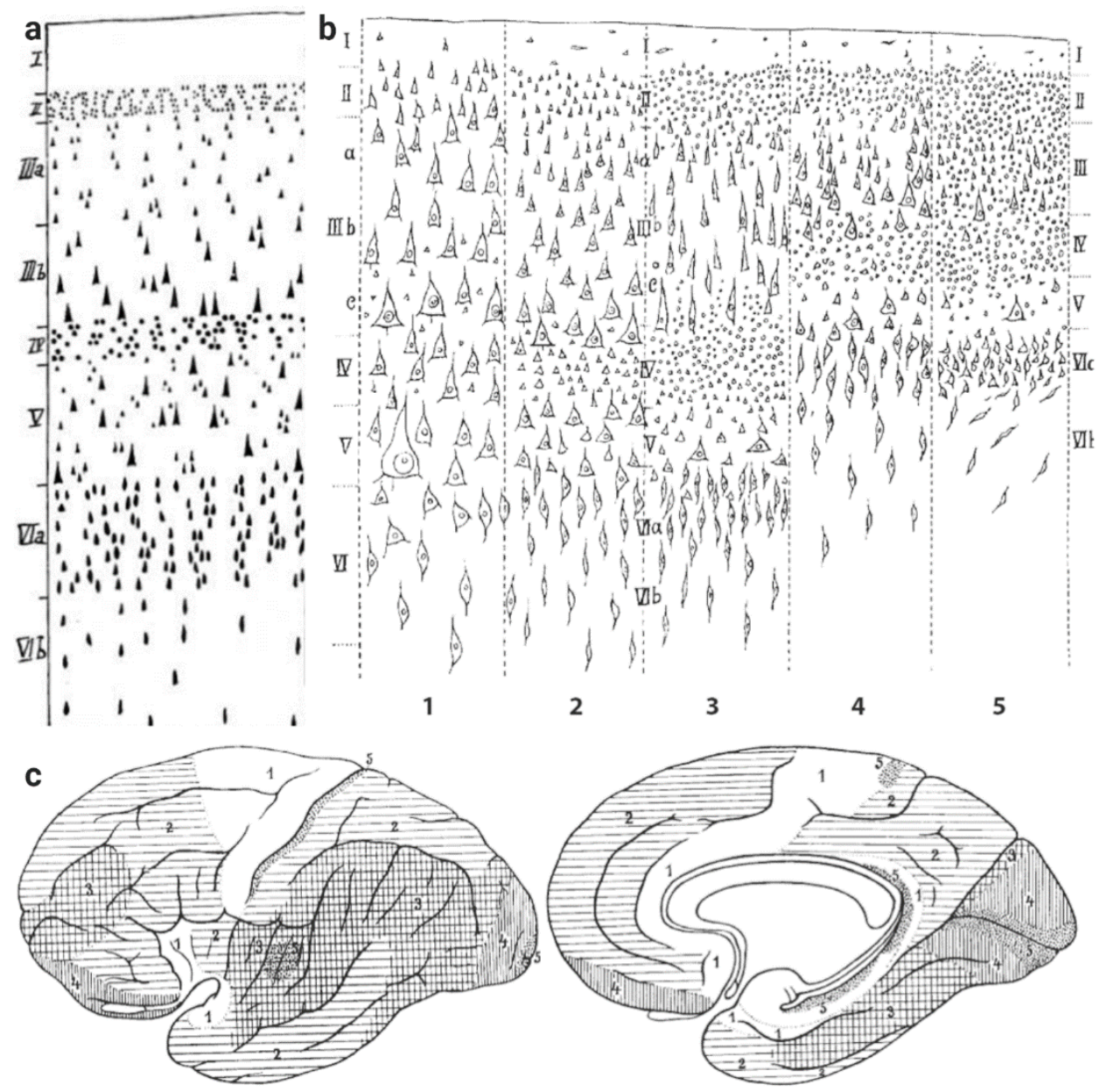

Figure 3: Basic plan of cytoarchitectural layers and their main variation. a)

Shows the idealized cytoarchitecture of the isocortex (adapted from Vogt, $\left.1910 b^{8}\right)$. b) This basic plan of the brain's cytoarchitecture shows three homotypical variations (2-4), which are predominantly found in the frontal, parietal, and polar regions of the cortex. The two heterotypical cortex types (1 and 5) are found primarily in motor areas and primary sensory areas, respectively. c) The distribution of these five main cytoarchitecture types on the brain is indicated in increasingly fine hatching ( $b$ and $\mathbf{c}$ modified from von Economo and Koskinas, 1925³). 
The second readily apparent feature in cortical cytoarchitecture is the vertical or radial cell arrangement (Fig 4) $)^{30-36}$. Although these arrangements are variably referred to in the literature as microcolumns, minicolumns or just columns, they will be referred to as minicolumns from here on. These structures are not identical with the larger and also variably named columns or macrocolumns observed in functional studies ${ }^{33,34,36}$. They arise during early corticogenesis through the radial migration of pyramidal cells. These early developmental structures of stacked pyramidal cells known as "ontogenetic columns" are considered the origin of minicolumns in the adult cortex ${ }^{37-39}$. Even with their functional importance in the adult brain still under debate ${ }^{33,35,40}$, they are a useful feature for the parcellation of cortical areas as they vary over different cortical regions (Fig. 4c). While it is not fully clear why minicolumns are more or less prominent in some areas compared to others, this could be seen as an indicator of their functional relevance. A prominent opinion is indeed that minicolumns are not merely structural artefacts from early development, but rather form the basic functional unit of the cortex $^{30-32,41,42}$. 

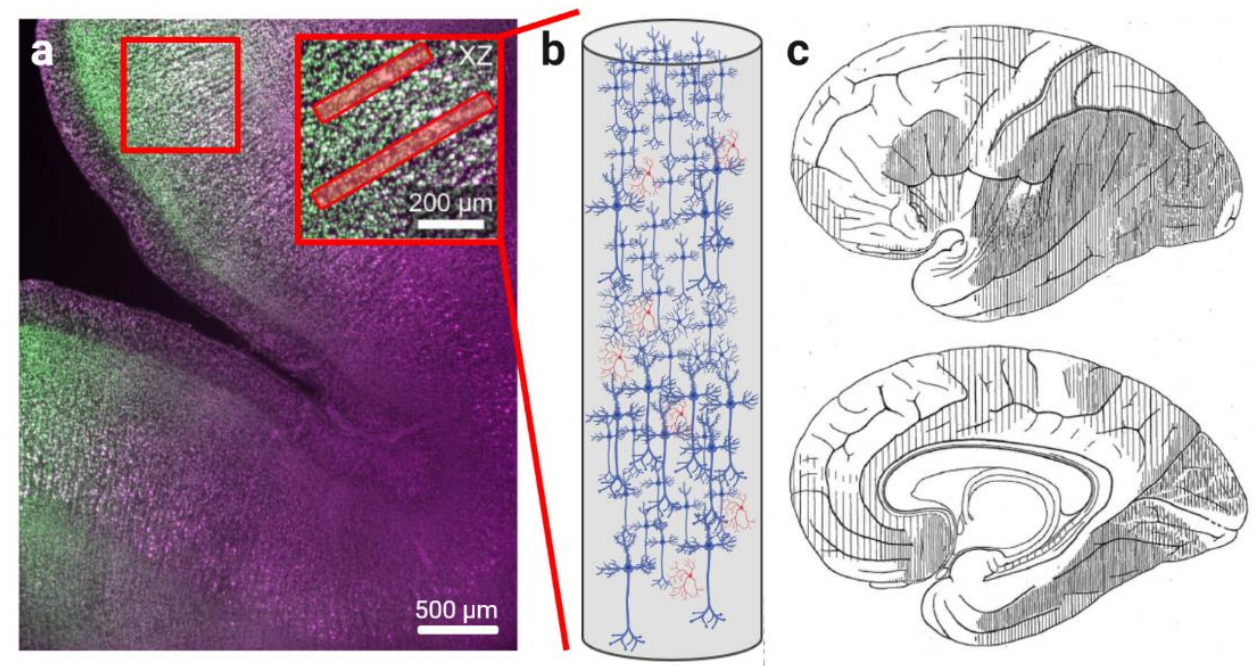

Figure 4: The organisation of the cortex into minicolumns. a) Light-sheet microscopy images of human occipital lobe samples stained for cytoarchitecture. (a MASH clearing and staining, see Chapter 2, imaging with the mesoSPIM light sheet, see Chapter 3). The enlarged insert shows minicolumns in the supragranular layers. b) Schematic drawing of one minicolumn over all layers with putative excitatory neurons incidated in blue and putative inhibitory neurons in red. c) Variations in the visibility of minicolumns across different regions. No hatching indicated no distinctly visible minicolumns, increasingly fine hatching increasing prominence of a columnar organisation (modified from von Economo and Koskinas, 1925³).

\section{Angioarchitecture}

Compared to cyto- and myeloarchitecture, the field of angioarchitecture has not received as much attention historically. Most early investigators focussed their attention on the nervous elements to gain knowledge on brain function, for obvious reasons. 
Nevertheless, there are some examples of early anatomists focussing on micro-vascular differences across the brain, realizing its crucial role in pathologies ${ }^{43-51}$. As with so many architectural aspects, the field of angioarchitecture was likewise first mentioned by the Vogts ${ }^{50,52}$. The true first pioneer of this field however was Richard Arwed Pfeiffer ${ }^{46-48,50}$. The study of human angioarchitecture could experience a renaissance, because functional imaging of the human brain using functional magnetic resonance imaging (fMRI) is pushed to ever increasing resolution ${ }^{53,54}$. With ultra-high field (UHF) MRI (at or beyond a field strength of $7 \mathrm{~T}$ ) mapping of the functional architecture becomes possible at mesoscopic scales ${ }^{55}$.

The signal measured with fMRI is intricately connected to the underlying vasculature ${ }^{56}$. In ultra-high field fMRI, the resolution is high enough to disentangle activity in laminar and columnar modules of the cortex. While not currently done, UHF MRI will make it possible to measure at scales at which intra- and inter-areal differences in the angioarchitecture may have to be taken into account during analysis. Post mortem studies of angioarchitecture on a microscopical level in 3D would provide an unprecedented scale of information that could further aid the interpretation of these signals. This is explored in Chapter 3 of this thesis. 

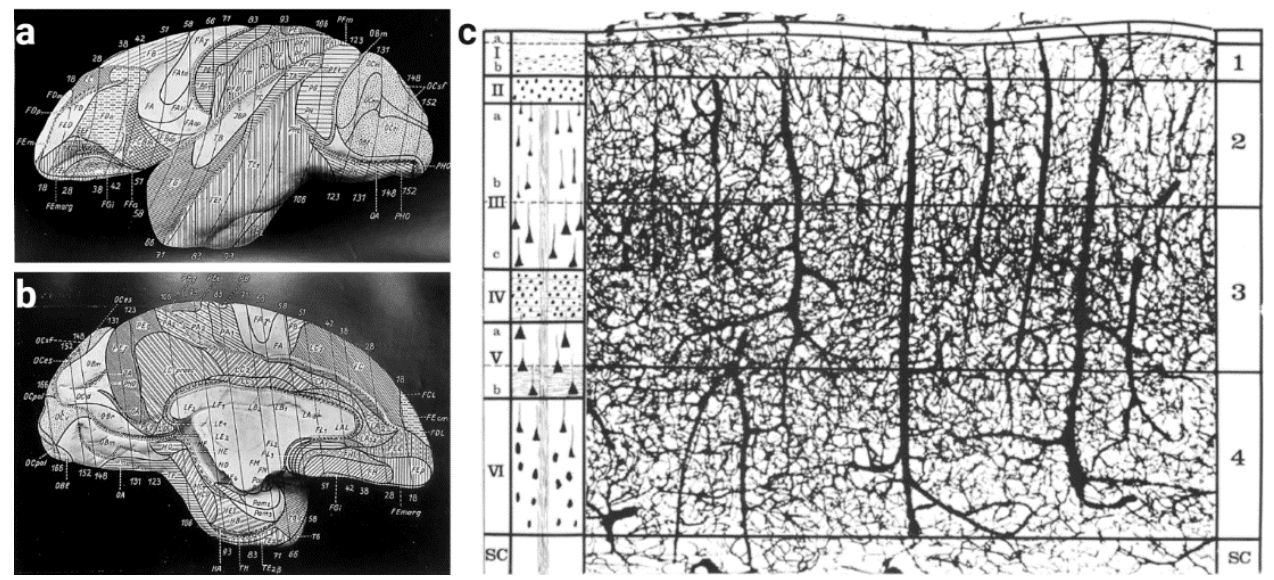

Figure 5: Brain parcellation using angioarchitecture. a) Lateral and b) medial view of the fully mapped rhesus macaque brain (modified from Pfeiffer, 194048). c) The four principle angioarchitectonic layers of Duvernoy and their relation to the cytoarchitecture (adapted from Duvernoy et al., 198156).

Although there has been monumental work on angioarchitecture by several investigators in primate and human brains ${ }^{46-48,57-62}$ (see Fig. 5), a complete map of the human angioarchitecture does not exist to this day. It seems to be clear however, that 1) different types of arteries and veins penetrate the cortex to various depths, 2) the dominant orientation changes over cortical layers and in white matter compared to grey matter, 3 ) the microvasculature is not constant over cortical depth resulting in a layered arrangement, and 4) microvasculature (the network capillaries, arterioles and venules) varies enough across the cortex to delineate different areas. 


\section{Limitations of classical brain parcellation}

A major limitation of all these brain maps, independent of the specific architecture, is the high variability between maps by different investigators. Even within the same architectural criteria, brain maps can differ substantially (see beginning of the chapter) and in some cases the whole brain has never been completely charted. The high variability between different brain maps was noted early on. Among the harshest critics where Bailey and von Bonin ${ }^{9}$, claiming that it is impossible to differentiate areas based on cytoarchitecture or myeloarchitecture. They concluded this after trying unsuccessfully to piece their own parcellation together in a puzzle of shuffled photomicrographs taken from different brain regions. Regarding the myeloarchitectonic cortex types of the Vogt school (Fig. 2), or the parcellation proposed by von Economo based on his five main types of cytoarchitecture (Fig. 3), they claimed: "The distribution of all these types, however, serves to emphasize a point to which we have repeatedly recurred, namely, the practical impossibility of distinguishing vast regions of the frontal, inferior parietal, parieto-occipital, occipitotemporal, basal temporal, and superior temporal regions. If we take once more our photographs of these regions without identifying marks and shuffle them, we are still unable to sort them by region." . They further show seemingly convincingly that the individual parcellations based on either cyto- or myeloarchitecture proposed by various neuroanatomists are inherently inconsitstent, which leads them to conclude: "For this reason we reject the excessive parcellations 
of the Vogt, Economo, and Filimonoff schools as misleading and insignificant. As one reads through their prolix descriptions, and has the misfortune to remember what he has read, one is either repeatedly shocked by contradictions or suffers from what the French psychiatrists call le phénomène du déjà-vu, description after description sounding merely like paraphrases of the preceding one." It is not without irony however, that their own map overlaps very well with the basic cytoarchitecture types defined by von Economo and Koskinas (compare Fig.1a with Fig.4c). An intriguing possibility is the connection of these most distinct cytoarchitecture zones with developmental gradients of transcription factors ${ }^{38,63-65}$ and the gradients observed in myeloarchitectonic development ${ }^{66,67}$.

Modern approaches for brain mapping avoid the subjective variability by applying observer-independent ways of drawing the boundaries and establishing probabilistic maps derived from many individuals rather than the idealized historical maps ${ }^{10,11,15,16,67,68}$. While these advances offer a huge leap forward for brain anatomy, they still work with the same thin $2 \mathrm{D}$ sections as the investigators of the early $20^{\text {th }}$ century. The $3 \mathrm{D}$ reconstruction of these thousands of sections into an unsheared, undistorted volume is extremely complex and computationally intense. Therefore, taking the imaging to thick optically cleared slices, which are much less prone to those distortions, and scanning them directly in 3D could potentially allow for faster and easier data acquisition and reconstruction. This could 
also allow smaller labs to do these kinds of studies, which are currently only feasible in large institutes with extensive infrastructure.

\section{Intrinsic cortical connectivity}

Intrinsic cortical connectivity in the human brain is very underinvestigated, because these connections are very hard to visualise. There is only one method really suitable to study intrinsic connectivity in the human and that is labelling with lipophilic carbocyanine and aminostyryl dyes ${ }^{69-75}$. This method, however, is not used very often as it requires very fresh and unfixed tissue, a special fixation with highly toxic chemicals, and very long incubation times of at least several months. For this reason, only few insights on intrinsic cortical connectivity have been directly gained for the human brain ${ }^{69,72-74}$ and most is translated from investigations in animals, which are more accessible for experimental manipulation.

Excitatory neurons form extensive collateral connections in many layers (Fig 6), which partly correspond to the horizontal layers, observed in myeloarchitecture described earlier (Fig. 2) ${ }^{76}$. Pyramidal neurons in layers II, III and $\mathrm{V}$ have dense local and sparser long (2-3 $\mathrm{mm}$ ) collaterals largely within the same layer. These collaterals do not spread out homogeneously, but form patches known as "daisies" ${ }^{\text {"76-78 }}$. Furthermore, they form vertical connections extending through layers I-V. The innervation targets of these patches seem to be diverse and not in a simple "like-to-like" relationship ${ }^{77}$. It seems plausible that the supragranular collaterals are partially responsible for the Kaes- 
Bechterew-stripe and layer $\mathrm{V}$ collaterals for the inner stripe of Baillarger. However, the majority of the axons in these stripes are likely extrinsic ${ }^{76}$. Neurons in layer IV (small pyramidal cells and, in the case of primary sensory areas, spiny stellates) also form horizontal collaterals. Spiny stellates seem to form more local collaterals within the same (vertical) functional domain, whereas pyramidal neurons spread across them. In the primary visual cortex extensive lateral connections in layer IVb (also patchy) give rise to the stripe of Gennari. Layer IV collaterals remain mainly in the parent layer, but also innervate layer III $^{79-82}$. Diverse populations on pyramidal neurons (corticothalamic, corticoclaustral, corticocortical) give rise to collaterals in layer VI as well. An exceptional example are the Meynert cells in primary visual cortex which collaterals can be $8 \mathrm{~mm}^{\text {long }}{ }^{76,83,84}$. Other layer VI collaterals form vertical branches as well in an areaspecific manner. A more recent discovery is a potential class of excitatory neurons in layer I of primates, the subpial fan cell, which forms long axonal arbores in that layer ${ }^{85}$.

The axonal arborisation of inhibitory neurons is highly complex and type dependent. Some cell types, such as the parvalbumin-positive basket cells can have extensive horizontally and vertically spreading arbores of several millimetre length and similar patchy arrangements as seen in pyramidal neurons ${ }^{86,87}$. A detailed account on axonal patterns of inhibitory neurons is beyond the scope of this introduction. 


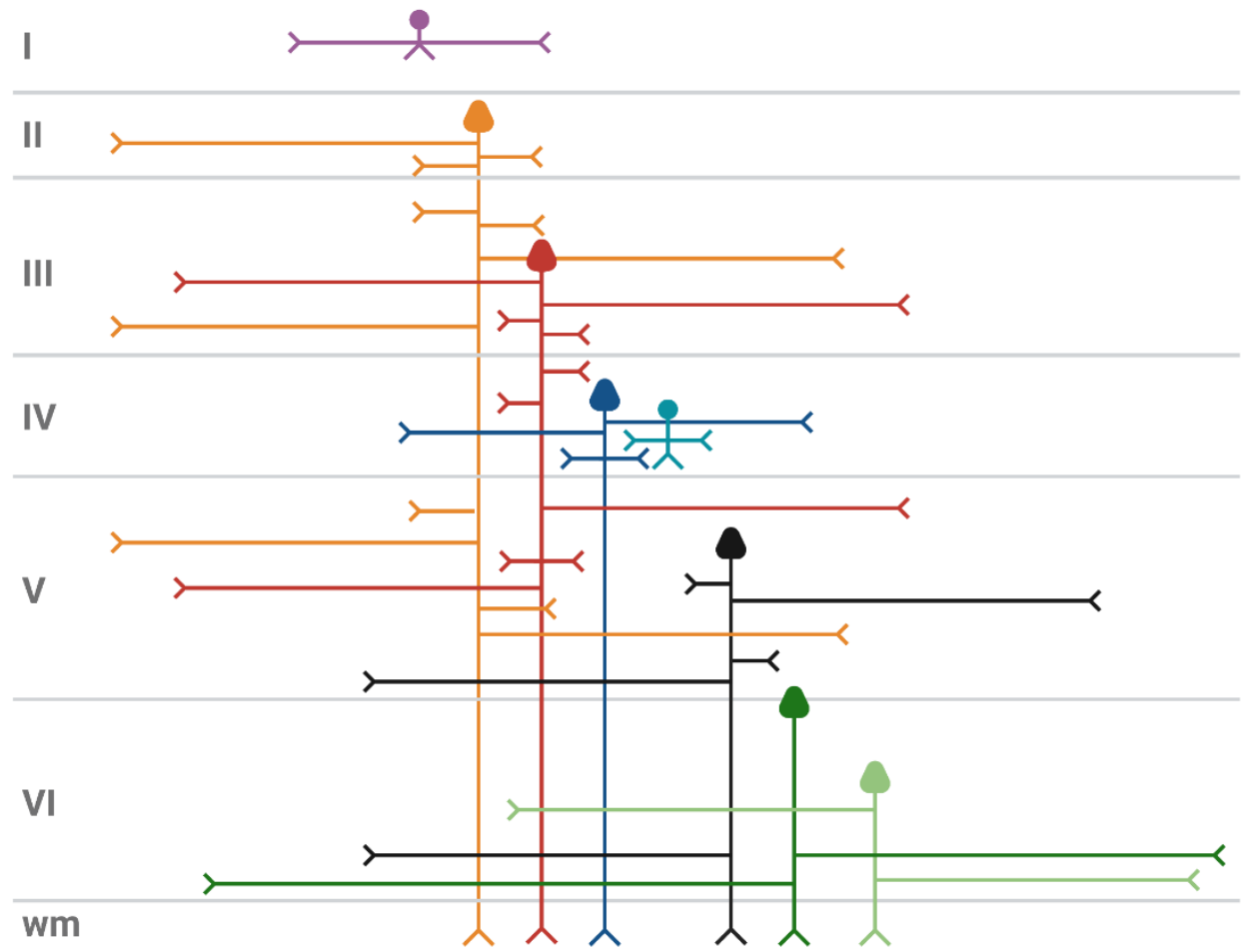

Figure 6: Origin of intrinsic horizontal collaterals by excitatory neurons. Neurons are listed from top to bottom and left to right as they appear in the figure: Subpial fan cells (purple), supragranular pyramidal neurons (red, orange), small granular pyramidal neurons (blue) and spiny stellates (turquoise), infragranular pyramidal neurons (black, green) with the special case of extremely long Meynert cell collaterals (dark green). The schematic is a simplification and does not show all the collaterals in an all-inclusive manner as this would be highly species- and area-dependent. It is also noted that merely the (main) horizontal origins of the collaterals from the main axons are shown for clarity and simplicity. Vertical branches extending over various layers can be formed by these horizontal branches as described briefly in the text. Note also that not all pyramidal neurons send main axons extra-areally through the white matter (wm). 
Despite of all that is known about intrinsic cortical connectivity, a lot is still unclear, especially in the human brain and outside the primary visual cortex. With the new technological developments described below, it is now becoming possible to study this in an unprecedented manner. These new methods could lead to a revival of lipophilic tracing studies in the human brain in the near future, once the last technological hurdles have been removed (see Chapter 5 for first steps in that direction).

\section{Tissue clearing: An overview}

One of the goals of anatomical studies is the investigation of tissues and organs without disrupting their integrity or the morphological relation to the whole body. This has led to the invention of tissue clearing, also called optical clearing, a procedure to make biological tissues optically transparent. Although this method has garnered renewed interest in recent years, it was invented more than 100 years ago. The first tissue clearing approach that gained more widespread use (and is still in use today for macroscale anatomical specimen), is the method developed by Werner Spalteholz in the beginning of the $20^{\text {th }}$ century (Fig. 7$)^{88-90}$. His protocols already contained all the major steps still present in their more modern variants and proved to be highly effective in its clearing capacity. However, this highly efficient clearing came at a price: the very harsh and often toxic chemicals tended to destroy the most superficial tissue layers. The destructive nature of the technique in combination with the lack of volumetric 
fluorescent microscopy explains why it took until the early $21^{\text {st }}$ century for the translation of these techniques to microscopic investigations of tissues.

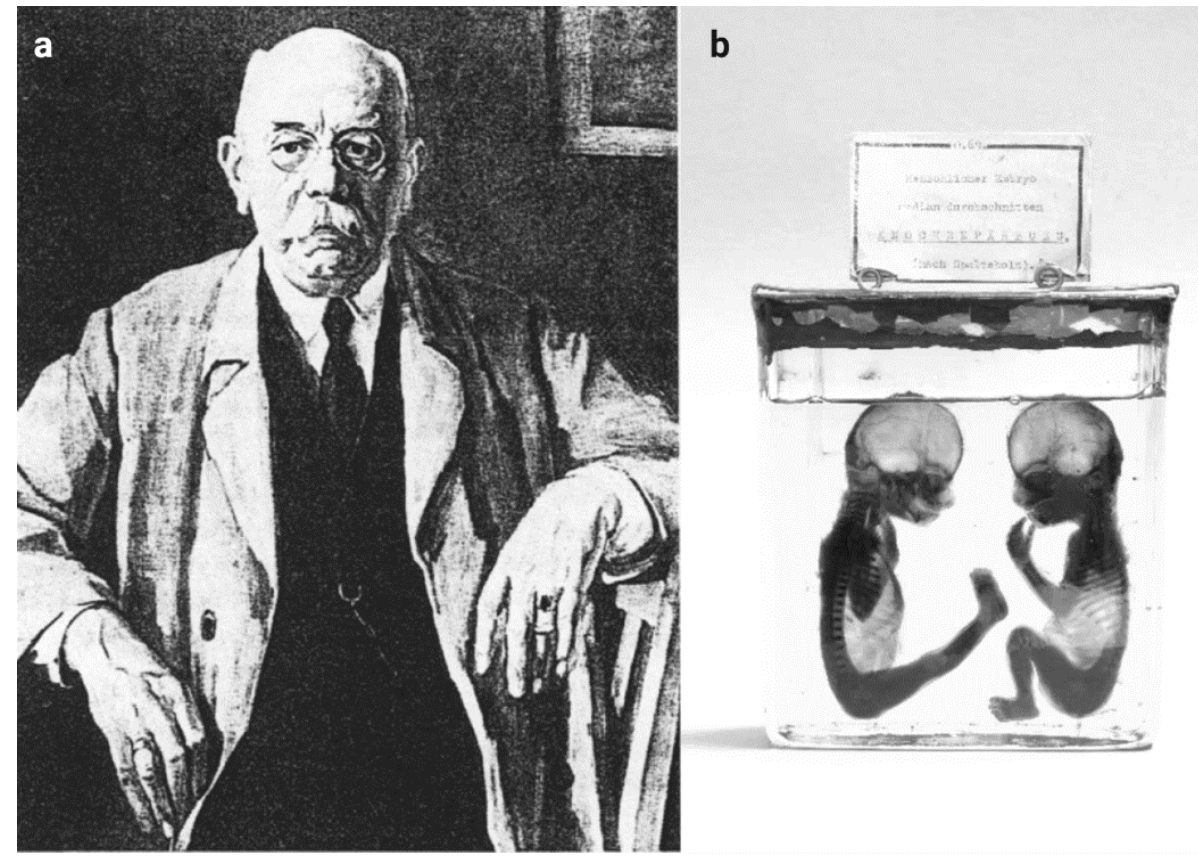

Figure 7: The inventor of tissue clearing. a) Portrait of Werner Spalteholz and b) mid-sagittal section of an approx. 22 week old human fetus treated with his original protocol (modified from Hahn, $1999^{86}$ and Ulrich, 201788, respectively).

Independent of the exact nature of the protocol, the steps to render tissues transparent are essentially the same (Fig. 8) ${ }^{91-93}$. Tissues are usually opaque because they 1) contain pigments, which absorb light to different degrees and 2) because the multitude of components in any biological tissue result in an unequal distribution of light 
scattering molecules. Conversely, this means that in order to render tissue transparent, it needs to be depigmented (bleached) and the light scattering has to be homogenised as much as possible. The latter is achieved by removing those tissue components, which differ the most from its proteins (usually the component of interest). In the particular case of the brain, this means removing the lipids (delipidation) and replacing the cytosol and extracellular fluid with a liquid of the same refractive index (RI) as the proteins. This step, called RI-matching, homogenises the distribution of scattering molecules and ultimately renders the tissue transparent.

\section{Bleaching Delipidation RI-matching}

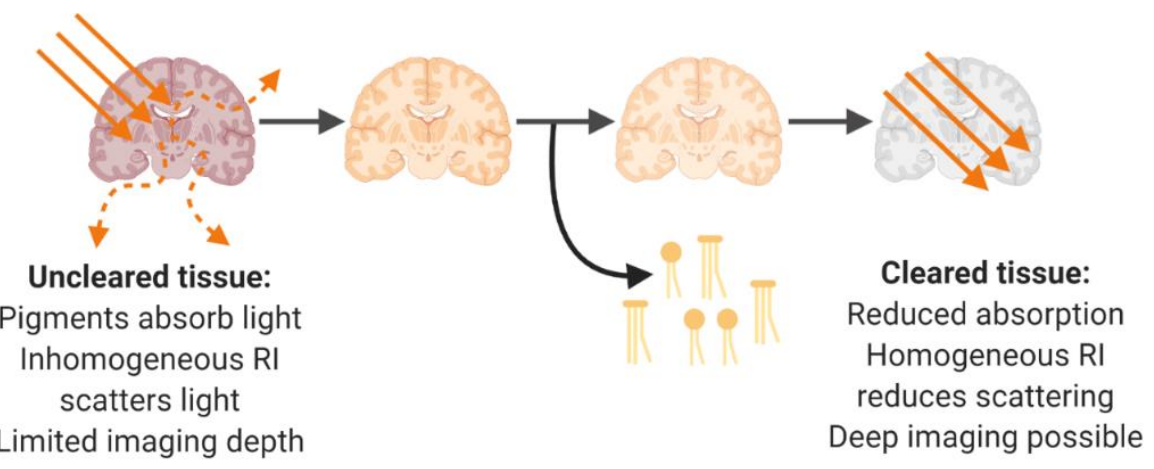

Figure 8: Basic steps of optical clearing protocols. Independent of the sample and specific clearing method, the opaque tissue is treated with the same steps. Absorbance is reduced by bleaching and $\mathrm{RI}$ inhomogeneities are reduced by delipidation and RI-matching. Depending on the tissue, certain parts of the pipeline can be more challenging than others. 
The main difference in clearing protocols is the environment in which these steps are carried out. Solvent-based protocols rely on alcohols and organic solvents or essential/ethereal oils ${ }^{94-99}$, whereas aqueous methods use water-soluble molecules and detergents if they aim at removing lipids ${ }^{100-105}$. A more detailed explanation on the different groups of clearing approaches is given below. Figure 9 depicts the development of ever more diverse and sophisticated ways of turning biological tissues transparent. Even a cursory glance at the current tissue clearing literature can be daunting, because of the multitude of protocols and the rate at which new ones are published. It is therefore beyond the scope of this general introduction to present a comprehensive account of all the various protocols. Rather, a general overview of the different groups of clearing methods and some examples that are more widely known are provided here.

\section{Solvent-based clearing protocols}

The rediscovery of solvent-based clearing for fluorescence microscopy led to a constant stream of publications on more and more fine-tuned and specialized protocols. Early uses of solventbased clearing for microscopic imaging date back at least to $1989^{106}$. However, the first wider attention in the international neuroscience community came after the 2007 publication by the Dodt group ${ }^{107}$. Since then, many adjustments have been made to the original formulation leading to a closely related family of clearing methods, often referred to as the DISCO family of clearing approaches after its 
most widely known versions ${ }^{97,98,108-116}$. Yet, the key steps and ingredients in these clearing protocols are essentially the same and differ mainly in the particular chemicals used for each step (Fig. 8).

Why is there no single best combination of chemicals for clearing? Why is there no obvious best clearing protocol? Depending on the kind of tissue under investigation, certain steps in the clearing pipeline will be more important than others. Experimenters investigating transgenic animals tagged with fluorescent proteins ideally want to preserve these proteins during clearing and therefore have to choose alcohols and solvents compatible with these proteins. Here tert-butanol and isopropanol buffered to a $\mathrm{pH}$ between 8 and 9 proved most effective (fluoroBABB ${ }^{99}$ and various DISCO protocols $\left.{ }^{110,111,116}\right)$. In other circumstances, such as in pathology, fast clearing is most essential which might necessitate harsher chemical treatments (e.g. pathoDISCO ${ }^{113}$ ). A downside, however, of fast dehydration protocols can be more pronounced tissue deformation. Depending on the underlying question of investigation, tissue shrinkage and deformation might be more or less problematic. Usually, one would expect that keeping the tissue as close to the precleared state as possible is desirable. In solvent-based approaches, this is currently achieved with the use of methanol for dehydration, which keeps tissue shrinkage at a minimum as originally introduced in iDISCO $+^{97,117}$. As noted above, this treatment will interfere with endogenous fluorescent labels and is therefore recommended for tissue without fluorescent proteins. Conversely, since 3D imaging of 
cleared samples collects large amounts of data, a strategy to mitigate this can be the intentional shrinkage of the tissue, so that less volume needs to be imaged in total. This has been used on entire mouse bodies in the $\mathrm{uDISCO}^{110}$ and $\mathrm{vDISCO}^{116}$ methods. Note that this strategy would sacrifice resolution as well, as fluorophores are moved closer together (see description of expansion microscopy below for the opposite effect). Finally, heavily pigmented tissue will need more elaborate bleaching ${ }^{118}$.

Overall, solvent-based clearing provides the most robust protocols with very high clearing capacity and good scalability and are hence a good starting point when targeting large human brain samples which are notoriously difficult to clear (see Chapter 2-4). 


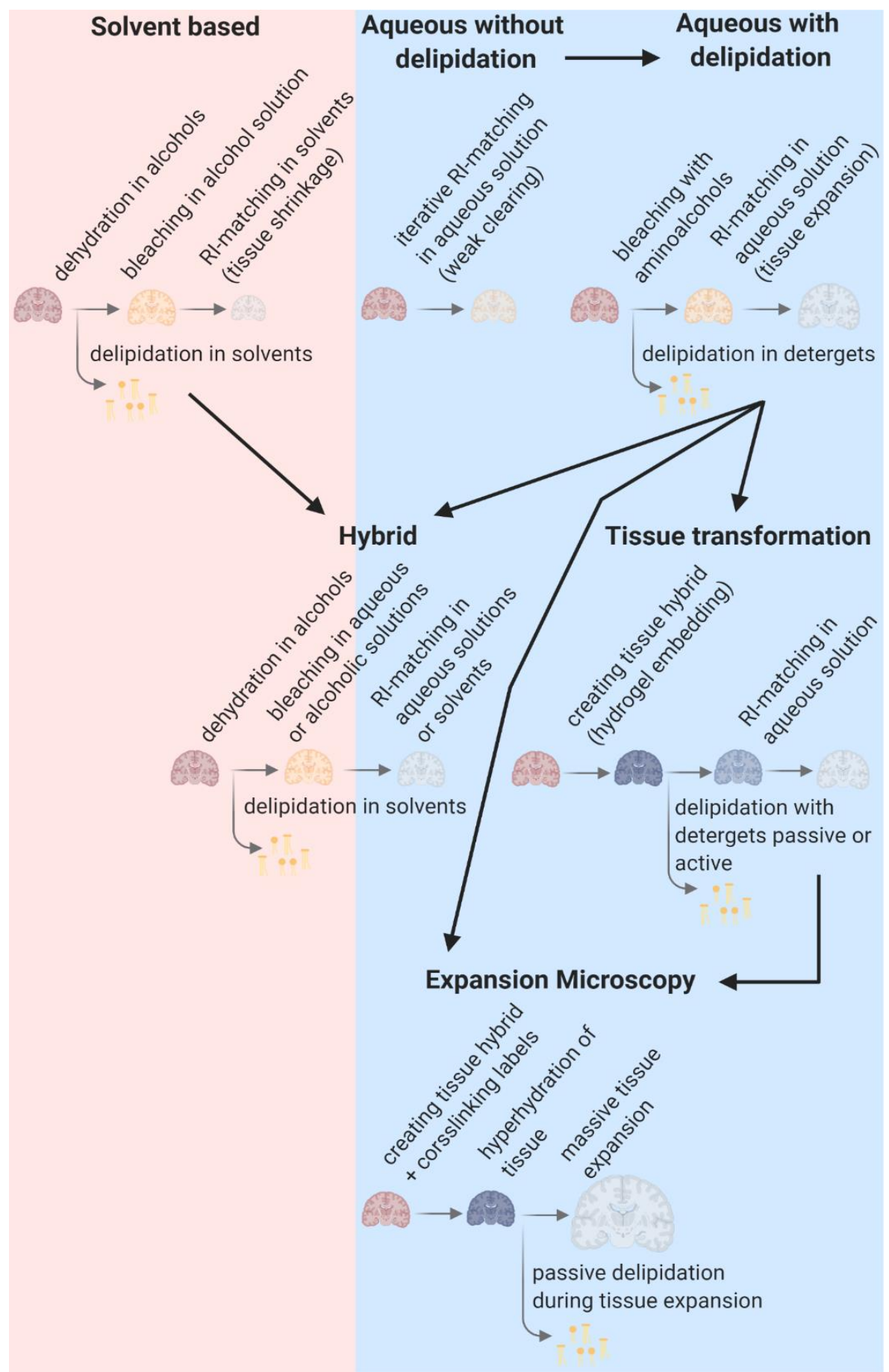


Figure 9: Main groups of optical clearing protocols and their relation to one another (prior page). Most larger developments have occurred with aqueous methods. While solvent-based approaches are continuously improved as well, the general concepts have been much more preserved.

\section{Aqueous clearing protocols}

Although aqueous clearing methods are often described in the literature as relatively recent inventions ${ }^{91}$, they actually have existed in niche applications for decades. In certain anatomical subdisciplines within e.g. embryology or ichthyology, glycerin-based clearing has been used for macroanatomical studies for at least 50 years ${ }^{19,120}$. These old techniques rely on many of the same steps and chemicals used today on modern protocols.

Within the modern aqueous clearing methods, one can roughly distinguish three different sub-groups. The first generation are simple immersion protocols without any delipidation of the tissue $\mathrm{e}^{101-104,121-124}$. While the omission of this step will always reduce the sample transparency and hence the imaging depth, it can still be relatively effective for smaller samples or tissues devoid of fat. Keeping the lipids intact can allow for special applications in combination with lipophilic dyes and tracers, not possible with any other clearing $\operatorname{approach}^{101,103,125,126}$ (see Chapter 5).

Shortly after the first appearance of these protocols, other clearing formulas were published, which use detergents for delipidation, 
thereby greatly increasing overall transparency ${ }^{105,125,127,128}$. However, compared to solvent-based clearing, the delipidation of the sample can take a very long time (up to several months) depending on the tissue type and size $\mathrm{e}^{125}$. This major disadvantage can be addressed by the addition of electrical currents ${ }^{100}$ or thermal energy ${ }^{129,130}$ to markedly decrease the delipidation time, but both approaches suffer from their own shortcomings with regards to user-friendliness, scalability or tissue integrity. Very recently, considerable progress has been made on improving passive delipidating aqueous protocols with many new chemicals identified after extensive screenings of thousands of compounds ${ }^{128,131,132}$.

In order to make the tissue stiffer and more resilient to harsh chemical treatments, a third group of protocols was invented and has quickly gained recognition in the neuroscience community. These methods are based on transforming the tissue into a hydrogel hybrid, by attaching macromolecules of interest (usually the proteins) to crosslinking chemicals, fixing them into a stable scaffold ${ }^{100,129,130,133-141}$. By carefully controlling the properties of the hydrogel and the surrounding medium, tissues can even be swollen up and expanded ${ }^{142-}$ 146. The widening of intermolecular distances not only benefits transparency (by filling the expanded tissue with water), but it also increases the optical resolution (by increasing the distance between two fluorophores). Additionally, samples can be imaged in water dipping objectives, which are more readily available than specialised clearing objectives for high RIs. Despite these advantages, the use of 
expansion microscopy is ultimately limited to relatively small samples as the physical enlargement of them leads to an equal increase in imaging time and data volume, which quickly becomes prohibitive.

\section{Hybrid clearing protocols}

With an ever growing landscape of clearing techniques, there are many chemicals available for particular tasks now, such as especially potent bleaching of certain pigments ${ }^{114,118,128,132}$ or hydrophilic compounds with high $\mathrm{RIs}^{128}$. With the main tissue processing steps being the same in most protocol groups (see Fig. 9), researchers are starting to see clearing more as a modular system, in which steps from different protocols and across groups can be assembled together. This creates hybrid clearing approaches, often tailored to very specific applications ${ }^{118,147-149}$. It is likely, that this will result in more optimized clearing compared to any general protocol. Hybrid clearing techniques are therefore considered to become more abundant over time.

\section{Staining}

An important topic connected with optical clearing, is the labelling of structures of interest. It has been shown that substantial clinically relevant information can be gained without any labelling by autofluorescence alone ${ }^{113}$. Although this might provide enough insight for some research questions, most will require the visualisation of specific targets. Here one faces a choice of either sticking with 
established labelling approaches and tailor the clearing around them or try to come up with a labelling strategy that is compatible with an established clearing method.

In human samples, the use of endogenous labels is obviously not possible and exogenous probes have to be introduced into the tissue to stain specific structures. The standard tool for labelling highly specific targets are immunohistochemistry and immunofluorescence ${ }^{150,151}$. Both rely on antibodies binding to the structure of interest and visualisation with either light absorbing reaction products (immunohistochemistry) or fluorophores (immunofluorescence). While this is a powerful strategy, its main disadvantage lies in the size of the antibody molecules. These large molecules do not penetrate well into the tissue, which is essential for deep 3D imaging in large cleared samples ${ }^{152}$. Although many different ways have been published to address this issue ${ }^{116,130,133,152-156}$, no single solution stands out so far and is getting universally adopted. The reduction of the molecular weight of antibodies by using only the functionally relevant parts for instance can solve the diffusion problem $^{116,152}$, but it also increases the costs making this approach economically unfeasible for large human samples (at least at this point; see Chapter 6 for a more detailed discussion).

An alternative strategy is to rely on small molecules for staining the tissue. These small compounds are used since the beginning of histology ${ }^{157}$. As Chapter 2 shows, their low molecular weight gives 
them advantages in tissue penetration, enabling deep labelling and many of these organic dyes, used traditionally in brightfield microscopy, are fluorescent as well. The downside of these dyes is that they are less specific, since their staining patterns are dictated by general chemical properties and their interaction with the tissue ${ }^{150,151}$. For instance, many positively charged molecules will stain negatively charged DNA and RNA molecules and negatively charged molecules will bind to positively charged proteins ${ }^{158-161}$. Hydrophobic dyes will preferentially bind to hydrophobic tissue components such as lipids and when applying multiple labels simultaneously, their relative size will also influence the staining pattern, because of the resulting different diffusion rates ${ }^{159,160}$. One advantage that many of the established histochemical stainings possess, is their compatibility with delipidating tissue clearing protocols: in classical 2D histology, tissue sections are often dehydrated, treated with organic solvents and finally mounted in resinous media such as Entellan® or Canada balsam $^{150,151}$. If a particular dye is preserved during this process on $2 \mathrm{D}$ sections, it is reasonable to assume it will be compatible with similar steps in thick samples treated with e.g., a solvent-based clearing method. This makes small molecule dyes from traditional histology a highly interesting target for the development of clearing compatible stainings. Of course, this necessitates that the research question can be answered with the more general labelling thus produced. 


\section{Light-sheet fluorescent microscopy: Fast volumetric imaging at microscopic resolution}

The concept of light-sheet fluorescence microscopy is not a new one. Indeed, a parallel can be observed to clearing techniques because the idea was first developed in the early $20^{\text {th }}$ century and later rediscovered and put to other uses ${ }^{107,162,163}$. Richard Adolf Zsigmondy and Henry Friedrich Wilhelm Siedentopf were the first to build an extreme angled version of darkfield illumination, in which the condenser is placed orthogonal to the imaging objective (Fig. 10) ${ }^{163}$. With this method, Zsigmondy could see the shadows of colloidal particles too small to be resolved by classical light microscopy. Hence, the new method was called "Ultramikroskopie", a term that has been reused by the Dodt group and some commercial versions of modern microscopes as well ${ }^{107}$. For his discoveries Zsigmondy (though not Siedentopf who was the physicist actually building the microscope) received the Nobel Prize in Chemistry in 1925. 


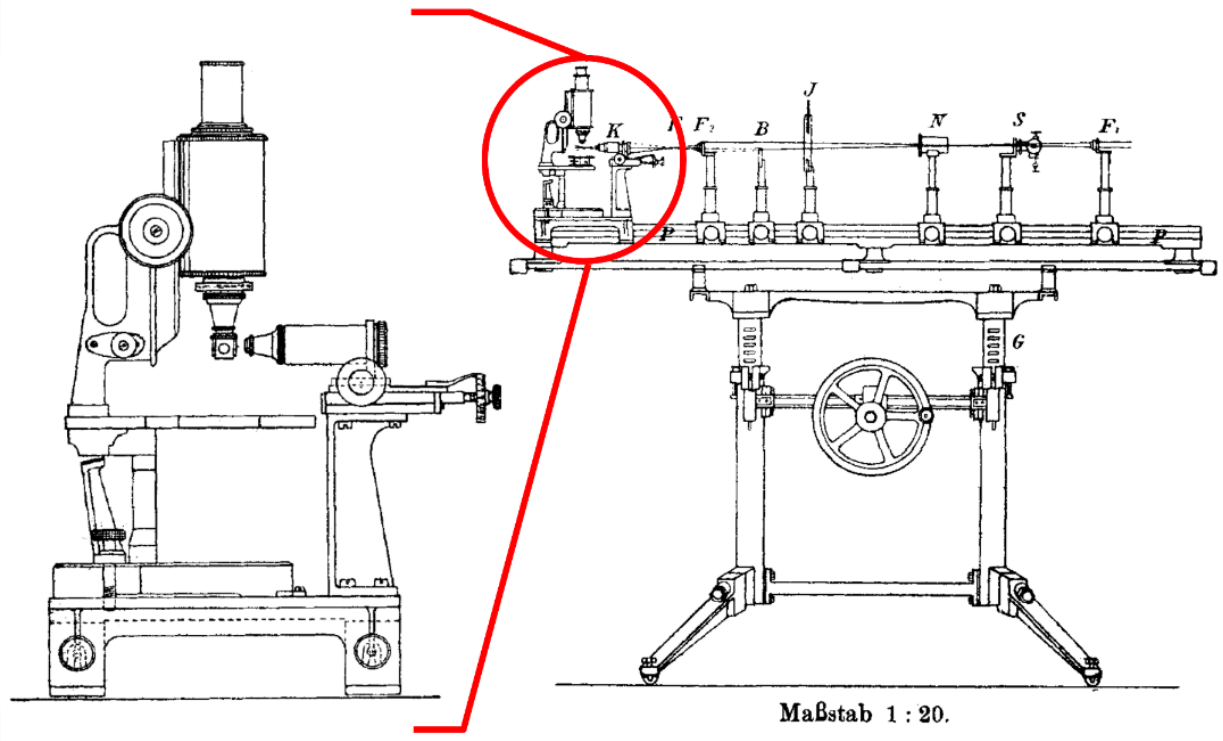

Figure 10: Design of the original "Ultramikroskop". Although the first versions used focused sun light, the geometry is essentially the same as in modern fluorescent light-sheet microscopes. Zsigmondy and Siedentopf even mention the use of their microscope on fluorescent solutions (modified from Siedentopf and Zsigmondy, 1903 $\left.{ }^{161}\right)$.

The described method was first used in a fluorescent microscope in 1993 to image guinea pig cochleae under the name "orthogonal-plane fluorescence optical sectioning" (OPFOS) ${ }^{164}$, which is the beginning of a second parallel to tissue clearing techniques: a confusing and everincreasing abundance of abbreviations. OPFOS is probably much less known than the two other synonyms "selective plane illumination microscopy" (SPIM) $)^{165}$ and "light-sheet fluorescence microscopy" $(\mathrm{LSFM})^{166,167}$. The latter term will be used for consistency in this text unless when referring to specific systems. The LSFM technique allows 
for fast volumetric imaging of transparent specimen as an entire plane of the sample is illuminated at a time $\mathrm{e}^{107,138,162,165-170}$. The simplest geometry of a light-sheet microscope is shown in figure 11a. Oblique geometries as used in the dual Selective Plane Illumination Microscopy for cleared tissue (ct-dSPIM) set-up (see Fig. 11b and Chapter 4), are more suitable for very large, flat samples. In these systems, the light does not have to penetrate the entire width of the sample to reach its centre and therefore, the lateral sample size is only restricted by the stage size and extent of movement.

The restriction of excitation light to a single plane also reduces phototoxicity in living specimen ${ }^{167,169}$. This and the fast acquisition of images makes it an ideal tool for developmental biology. Many of the model organisms in this field such as small drosophila or zebra fish embryos are inherently transparent. For other applications, the synergistic potential between optical clearing and LSFM was quickly recognized and exploited ${ }^{107}$. As this thesis will show, the limits for this synergistic potential are not yet reached by far, and it is expected that future developments in both fields will massively enhance the neuroanatomical understanding of the human brain (see Chapters 3 and 4). 


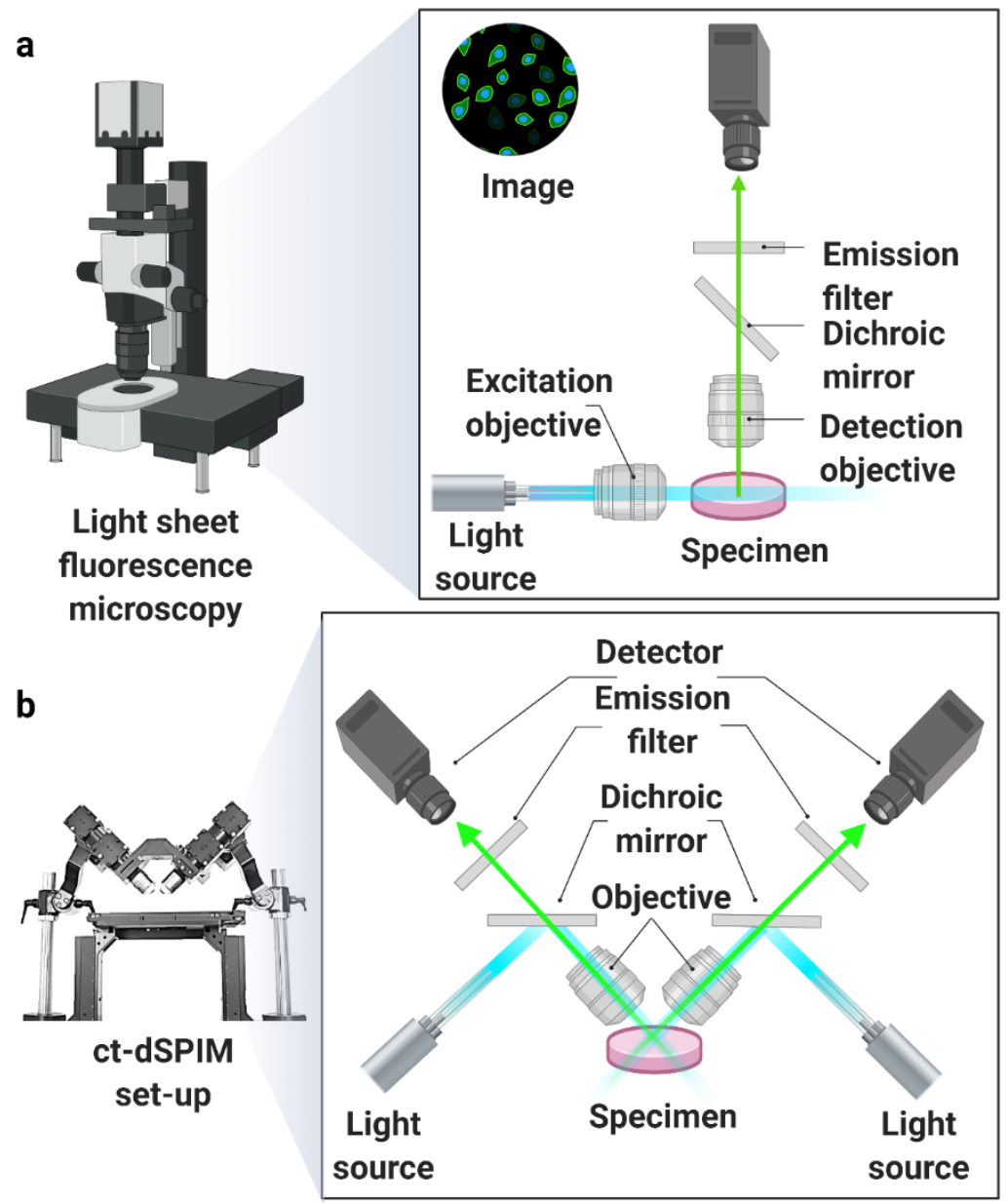

Figure 11: Design principles of fluorescent light-sheet microscopes. a) Basic design used in early versions with one excitation side and one detection side. b) Oblique geometry as used in e.g. the ct-dSPIM set-up is more suited for imaging of large cleared samples as compared to the classical set-up. 


\section{Aim of this thesis}

The aim of the work reported in this thesis lays primarily in the method development for 3D investigation of cortical architecture (Fig. 12). These developments focused on stainings for three different target structures: cytoarchitecture, angioarchitecture and intrinsic cortical connectivity. Additionally, both solvent-based and aqueous clearing protocols were optimized for their application on human brain tissue and advances in light-sheet microscopy for 3D imaging of large tissue volumes are introduced.

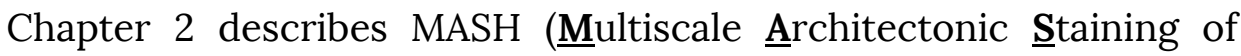
Human cortex), a novel combination of solvent based clearing with several economic dyes for labelling cytoarchitature in large human brain pieces.

In Chapter 3 the introduction of a staining method for angioarchitecture compatible with MASH allows for the visualisation and analysis of both angio- and cytoarchitecture in the same samples. Chapter 4 shows an up-scaled version of MASH to create a highthroughput pipeline, capable of processing entire human occipital lobes. The potential for this pipeline to be adjusted to accommodate even larger brain samples or sections of large organs other than the brain is discussed.

Chapter 5 extends the high-throughput MASH pipeline to formalinfixed paraffin-embedded (FFPE) prostate tissue and most importantly, 
introduces the ct-dSPIM, a new light-sheet microscope prototype optimized for imaging of very large, thick, and cleared specimen.

Finally, the development of hFRUIT, an optimized aqueous clearing protocol which can preserve lipophilic labels tracing the intrinsic cortical connectivity, while also being able to clear human grey mattter, is presented in Chapter 6 .

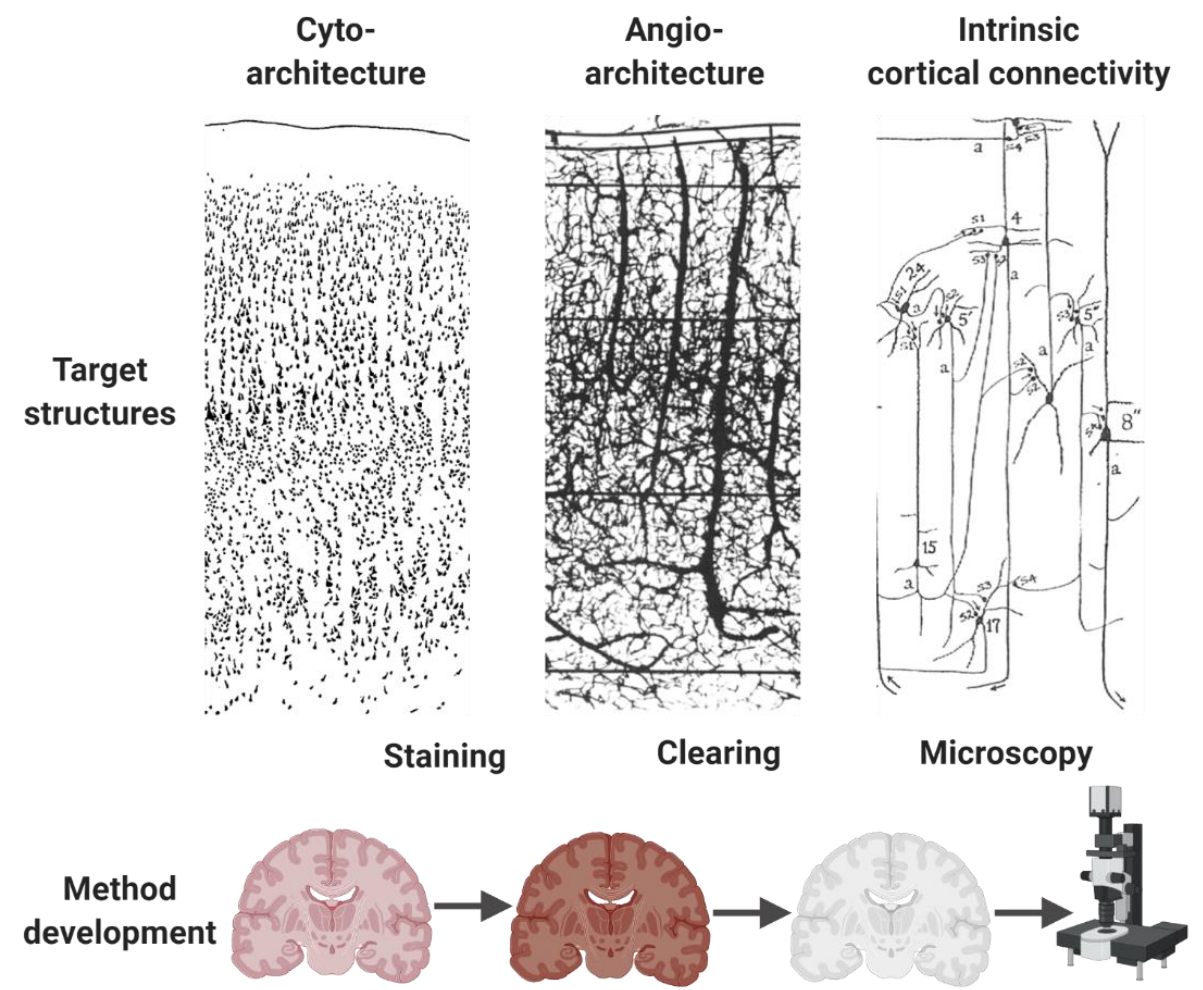

Figure 12: Main advances presented in this thesis. Methodological improvements regarding clearing \& staining (Chapters 2,3,4,6) and microscopy (Chapter 3,5$)$ have been developed to improve the investigation in three neuroanatomical fields: cytoarchitecture (Chapter 2,4,5), angioarchitecture (Chapter 3) and intrinsic cortical connectivity (Chapter 6). 


\section{References}

1 Brodmann, K. Vergleichende Lokalisationslehre der Grosshirnrinde in ihren Prinzipien dargestellt auf Grund des Zellenbaues. (Barth, 1909).

2 Campbell, A. W. Histological Studies on the Localisation of Cerebral Function. Journal of Mental Science 50, 651-662, doi:10.1192/bjp.50.211.651 (1904).

3 von Economo, C. \& Koskinas, G. N. Die Cytoarchitektonik der Hirnrinde des erwachsenen Menschen: Textband u. Atlas. (Springer, 1925).

4 Gomes, M. D. M. Franz Nissl (1860-1919), noted neuropsychiatrist and neuropathologist, staining the neuron, but not limiting it. Dementia \& neuropsychologia 13, 352-355, doi:10.1590/1980-57642018dn13030014 (2019).

5 Amunts, K., Schleicher, A. \& Zilles, K. Cytoarchitecture of the cerebral cortex--more than localization. Neuroimage 37, 1061-1065; discussion 1066-1068, doi:10.1016/j.neuroimage.2007.02.037 (2007).

6 Amunts, K. \& Zilles, K. Architectonic Mapping of the Human Brain beyond Brodmann. Neuron 88, 1086-1107, doi:10.1016/j.neuron.2015.12.001 (2015).

7 Nieuwenhuys, R. The myeloarchitectonic studies on the human cerebral cortex of the Vogt-Vogt school, and their significance for the interpretation of functional neuroimaging data. Brain structure \& function 218, 303-352, doi:10.1007/s00429-012-0460-z (2013).

8 Vogt, O. Die myeloarchitektonische Felderung des menschlichen Stirnhirns. J Psychol Neurol 15, 221-232 (1910).

9 Bailey, P. \& von Bonin, G. The Isocortex of Man. University of Illinois. Urbana (1951).

10 Amunts, K. et al. BigBrain: An Ultrahigh-Resolution 3D Human Brain Model. Science 340, 1472, doi:10.1126/science.1235381 (2013).

11 Amunts, K., Mohlberg, H., Bludau, S. \& Zilles, K. Julich-Brain: A 3D probabilistic atlas of the human brain's cytoarchitecture. Science 369,988 , doi:10.1126/science.abb4588 (2020). 
12 Glasser, M. F. et al. A multi-modal parcellation of human cerebral cortex. Nature 536, 171-178, doi:10.1038/nature18933 (2016).

13 Zilles, K. \& Amunts, K. Centenary of Brodmann's map--conception and fate. Nature reviews. Neuroscience 11, 139-145, doi:10.1038/nrn2776 (2010).

14 Zilles, K. \& Palomero-Gallagher, N. Cyto-, myelo-, and receptor architectonics of the human parietal cortex. Neuroimage 14, S8-20, doi:10.1006/nimg.2001.0823 (2001).

15 Schleicher, A., Amunts, K., Geyer, S., Morosan, P. \& Zilles, K. Observerindependent method for microstructural parcellation of cerebral cortex: A quantitative approach to cytoarchitectonics. Neuroimage 9, 165-177, doi:DOI 10.1006/nimg.1998.0385 (1999).

16 Schleicher, A. \& Zilles, K. A Quantitative Approach to Cytoarchitectonics - Analysis of Structural Inhomogeneities in Nervous-Tissue Using an Image Analyzer. J Microsc-Oxford 157, 367-381, doi:DOI 10.1111/j.13652818.1990.tb02971.x (1990).

17 Alkemade, A. et al. 7 Tesla MRI Followed by Histological 3D Reconstructions in Whole-Brain Specimens. Frontiers in neuroanatomy 14, 68 (2020).

18 Glasser, M. F., Goyal, M. S., Preuss, T. M., Raichle, M. E. \& Van Essen, D. C. Trends and properties of human cerebral cortex: correlations with cortical myelin content. Neuroimage 93 Pt 2, 165-175, doi:10.1016/j.neuroimage.2013.03.060 (2014).

19 Vogt, C. \& Vogt, O. Allgemeine ergebnisse unserer hirnforschung. Vol. 21 (JA Barth, 1919).

20 Nieuwenhuys, R. \& Broere, C. A. J. A map of the human neocortex showing the estimated overall myelin content of the individual architectonic areas based on the studies of Adolf Hopf. Brain Structure and Function 222, 465-480, doi:10.1007/s00429-016-1228-7 (2017).

21 Nieuwenhuys, R. \& Broere, C. A. J. A detailed comparison of the cytoarchitectonic and myeloarchitectonic maps of the human neocortex produced by the Vogt-Vogt school. Brain structure \& function 225, 27172733, doi:10.1007/s00429-020-02150-2 (2020). 
22 Nieuwenhuys, R., Broere, C. A. J. \& Cerliani, L. A new myeloarchitectonic map of the human neocortex based on data from the Vogt-Vogt school. Brain Structure and Function 220, 2551-2573, doi:10.1007/s00429-0140806-9 (2015).

23 Hopf, A. Über die Verteilung myeloarchitektonischer Merkmale in der Stirnhirnrinde beim Menschen. J Hirnforsch 2, 311-333 (1956).

24 Judaš, M., Cepanec, M. \& Sedmak, G. Brodmann's map of the human cerebral cortex - or Brodmann's maps? Translational Neuroscience 3, doi:10.2478/s13380-012-0009-x (2012).

25 Brodmann, K. in Handbuch der Neurologie 206-307 (Springer, 1910).

26 Insausti, R., Muñoz-López, M., Insausti, A. M. \& Artacho-Pérula, E. The Human Periallocortex: Layer Pattern in Presubiculum, Parasubiculum and Entorhinal Cortex. A Review. Frontiers in neuroanatomy 11, 84 (2017).

27 Rockland, K. S. \& DeFelipe, J. Editorial: Why Have Cortical Layers? What Is the Function of Layering? Do Neurons in Cortex Integrate Information Across Different Layers? Frontiers in neuroanatomy 12, 78 (2018).

28 Shepherd, G. M. The microcircuit concept applied to cortical evolution: from three-layer to six-layer cortex. Frontiers in neuroanatomy 5, 30, doi:10.3389/fnana.2011.00030 (2011).

29 Shepherd, G. M. \& Rowe, T. B. Neocortical Lamination: Insights from Neuron Types and Evolutionary Precursors. Frontiers in neuroanatomy 11, 100 (2017).

30 Peters, A. \& Sethares, C. Myelinated axons and the pyramidal cell modules in monkey primary visual cortex. Journal of Comparative Neurology 365, 232-255, doi:https://doi.org/10.1002/(SICI)10969861(19960205)365:2<232::AID-CNE3>3.0.CO;2-6 (1996).

31 Buxhoeveden, D. P. \& Casanova, M. F. The minicolumn and evolution of the brain. Brain, behavior and evolution 60, 125-151, doi:10.1159/000065935 (2002).

32 Buxhoeveden, D. P. \& Casanova, M. F. The minicolumn hypothesis in neuroscience. Brain : a journal of neurology 125, 935-951, doi:10.1093/brain/awf110 (2002). 
33 Molnár, Z. \& Rockland, K. S. Cortical columns. 103-126, doi:10.1016/b978-0-12-814411-4.00005-6 (2020).

34 Mountcastle, V. B. The columnar organisation of the neocortex. Brain : a journal of neurology 120, 701-722, doi:10.1093/brain/120.4.701 (1997).

35 Rockland, K. S. Five points on columns. Frontiers in neuroanatomy 4, 22, doi:10.3389/fnana.2010.00022 (2010).

36 Szentágothai, J. The 'module-concept' in cerebral cortex architecture. Brain Research 95, 475-496, doi:https://doi.org/10.1016/00068993(75)90122-5 (1975).

37 Marin, O. \& Rubenstein, J. L. Cell migration in the forebrain. Annual review of neuroscience 26, 441-483, doi:10.1146/annurev.neuro.26.041002.131058 (2003).

38 Rakic, P. Specification of cerebral cortical areas. Science 241, 170, doi:10.1126/science.3291116 (1988).

39 Rakic, P. Radial versus tangential migration of neuronal clones in the developing cerebral cortex. Proc Natl Acad Sci U S A 92, 11323-11327, doi:10.1073/pnas.92.25.11323 (1995).

40 Horton, J. C. \& Adams, D. L. The cortical column: a structure without a function. Philosophical transactions of the Royal Society of London. Series B, Biological sciences 360, 837-862, doi:10.1098/rstb.2005.1623 (2005).

41 Buxhoeveden, D. P., Switala, A. E., Roy, E., Litaker, M. \& Casanova, M. F. Morphological differences between minicolumns in human and nonhuman primate cortex. American journal of physical anthropology 115, 361-371, doi:10.1002/ajpa.1092 (2001).

42 Casanova, M. F., El-Baz, A. \& Switala, A. Laws of conservation as related to brain growth, aging, and evolution: symmetry of the minicolumn. Frontiers in neuroanatomy 5, 66, doi:10.3389/fnana.2011.00066 (2011).

43 de Crinis, M. in Anatomie der Sehrinde 33-36 (Springer, 1938).

44 Denkhaus, R. Über die Angioarchitektonik des Nucleus hypothalamicus. Archiv für Psychiatrie und Nervenkrankheiten 115, 61-81, doi:10.1007/BF01819398 (1942). 
45 Finley, K. H. ANGIO-ARCHITECTURE OF THE SUBSTANTIA NIGRA AND ITS PATHOGENIC SIGNIFICANCE. Archives of Neurology \& Psychiatry 36, 118-127, doi:10.1001/archneurpsyc.1936.02260070126010 (1936).

46 Pfeifer, R. A. Die Angioarchitektonik der Grosshirnrinde. (J. Springer, 1928).

47 Pfeifer, R. A. Grundlegende Untersuchungen für die Angioarchitektonik des menschlichen Gehirns. (J. Springer, 1930).

48 Pfeifer, R. A. Die angioarchitektonische areale gliederung der grosshirnrinde: auf grund vollkommener gefässinjektionspräparate vom gehirn des macacus rhesus. (G. Thieme, 1940).

49 Pfeifer, R. A. Myelogenetisch-Anatomische Untersuchungen über den Zentralen Abschnitt der Sehleitung. (Springer Berlin Heidelberg, 2013).

50 Engelhardt, F. Über die Angioarchitektonik der hypophysärhypothalamischen Systeme. Acta neurovegetativa 13, 129-170 (1956).

51 Schlesinger, B. The angioarchitecture of the thalamus in the rabbit. Journal of anatomy 75, 176-196 (1941).

52 Vogt, C. \& Vogt, O. in Nervensystem 448-477 (Springer, 1928).

53 Schmid, F., Barrett, M. J. P., Jenny, P. \& Weber, B. Vascular density and distribution in neocortex. Neurolmage 197, 792-805, doi:https://doi.org/10.1016/j.neuroimage.2017.06.046 (2019).

$54 \quad$ Uludağ, K. \& Blinder, P. Linking brain vascular physiology to hemodynamic response in ultra-high field MRI. Neurolmage 168, 279-295, doi:https://doi.org/10.1016/j.neuroimage.2017.02.063 (2018).

55 Dumoulin, S. O., Fracasso, A., van der Zwaag, W., Siero, J. C. W. \& Petridou, N. Ultra-high field MRI: Advancing systems neuroscience towards mesoscopic human brain function. Neurolmage 168, 345-357, doi:https://doi.org/10.1016/j.neuroimage.2017.01.028 (2018).

56 Ogawa, S., Lee, T. M., Kay, A. R. \& Tank, D. W. Brain magnetic resonance imaging with contrast dependent on blood oxygenation. Proceedings of the National Academy of Sciences 87, 9868, doi:10.1073/pnas.87.24.9868 (1990). 
57 Adams, D. L., Piserchia, V., Economides, J. R. \& Horton, J. C. Vascular Supply of the Cerebral Cortex is Specialized for Cell Layers but Not Columns. Cerebral cortex 25, 3673-3681, doi:10.1093/cercor/bhu221 (2015).

58 Duvernoy, H. M., Delon, S. \& Vannson, J. L. Cortical blood vessels of the human brain. Brain Research Bulletin 7, 519-579, doi:https://doi.org/10.1016/0361-9230(81)90007-1 (1981).

59 Weber, B., Keller, A. L., Reichold, J. \& Logothetis, N. K. The microvascular system of the striate and extrastriate visual cortex of the macaque. Cerebral cortex 18, 2318-2330, doi:10.1093/cercor/bhm259 (2008).

60 Cassot, F., Lauwers, F., Fouard, C., Prohaska, S. \& Lauwers-Cances, V. A Novel Three-Dimensional Computer-Assisted Method for a Quantitative Study of Microvascular Networks of the Human Cerebral Cortex. Microcirculation 13, 1-18, doi:https://doi.org/10.1080/10739680500383407 (2006).

61 Lauwers, F., Cassot, F., Lauwers-Cances, V., Puwanarajah, P. \& Duvernoy, $\mathrm{H}$. Morphometry of the human cerebral cortex microcirculation: General characteristics and space-related profiles. Neurolmage 39, 936-948, doi:https://doi.org/10.1016/j.neuroimage.2007.09.024 (2008).

62 Risser, L., Plouraboué, F., Cloetens, P. \& Fonta, C. A 3D-investigation shows that angiogenesis in primate cerebral cortex mainly occurs at capillary level. International Journal of Developmental Neuroscience 27, 185-196, doi:https://doi.org/10.1016/j.ijdevneu.2008.10.006 (2009).

63 Buckner, R. L. \& Krienen, F. M. The evolution of distributed association networks in the human brain. Trends in cognitive sciences 17, 648-665 (2013).

64 Rosa, M. G. P. Visual maps in the adult primate cerebral cortex: some implications for brain development and evolution. Brazilian Journal of Medical and Biological Research 35, 1485-1498 (2002).

65 Sansom, S. N. \& Livesey, F. J. Gradients in the brain: the control of the development of form and function in the cerebral cortex. Cold Spring Harbor perspectives in biology 1, a002519 (2009). 
66 Bourne, J. A. \& Rosa, M. G. P. Hierarchical development of the primate visual cortex, as revealed by neurofilament immunoreactivity: early maturation of the middle temporal area (MT). Cerebral cortex 16, 405-414 (2006).

67 Flechsig, P. E. Meine myelogenetische Hirnlehre: mit biographischer Einleitung. (Springer-Verlag, 2013).

68 Wagstyl, K. et al. Mapping Cortical Laminar Structure in the 3D BigBrain. Cerebral cortex 28, 2551-2562, doi:10.1093/cercor/bhy074 (2018).

69 Mufson, E. J., Brady, D. R. \& Kordower, J. H. Tracing neuronal connections in postmortem human hippocampal complex with the carbocyanine dye Dil. Neurobiology of aging 11, 649-653, doi:https://doi.org/10.1016/0197-4580(90)90031-T (1990).

70 Molnár, Z., Blakey, D., Bystron, I. \& Carney, R. S. E. in Neuroanatomical Tract-Tracing 3: Molecules, Neurons, and Systems (eds Laszlo Zaborszky, Floris G. Wouterlood, \& José Luis Lanciego) 366-393 (Springer US, 2006).

71 Honig, M. G. \& Hume, R. I. Dil and DiO: versatile fluorescent dyes for neuronal labelling and pathway tracing. Trends in neurosciences 12, 333341, doi:https://doi.org/10.1016/0166-2236(89)90040-4 (1989).

72 Burkhalter, A. \& Bernardo, K. L. Organisation of corticocortical connections in human visual cortex. Proceedings of the National Academy of Sciences 86, 1071, doi:10.1073/pnas.86.3.1071 (1989).

73 Burkhalter, A., Bernardo, K. L. \& Charles, V. Development of local circuits in human visual cortex. The Journal of Neuroscience 13, 1916, doi:10.1523/JNEUROSCI.13-05-01916.1993 (1993).

74 Galuske, R. A. W., Schlote, W., Bratzke, H. \& Singer, W. Interhemispheric Asymmetries of the Modular Structure in Human Temporal Cortex. Science 289, 1946, doi:10.1126/science.289.5486.1946 (2000).

75 Lanciego, J. L. \& Wouterlood, F. G. A half century of experimental neuroanatomical tracing. Journal of chemical neuroanatomy 42, 157-183, doi:10.1016/j.jchemneu.2011.07.001 (2011).

76 Rockland, K. S. What do we know about laminar connectivity? Neuroimage 197, 772-784, doi:10.1016/j.neuroimage.2017.07.032 (2019). 
77 Koestinger, G., Martin, K. A. C., Roth, S. \& Rusch, E. S. Synaptic connections formed by patchy projections of pyramidal cells in the superficial layers of cat visual cortex. Brain structure \& function 222, 30253042, doi:10.1007/s00429-017-1384-4 (2017).

78 Ojima, H., Honda, C. N. \& Jones, E. Patterns of Axon Collateralization of Identified Supragranular Pyramidal Neurons in the Cat Auditory Cortex. Cerebral cortex 1, 80-94, doi:10.1093/cercor/1.1.80 (1991).

79 Casagrande, V. A. \& Kaas, J. H. in Primary Visual Cortex in Primates (eds Alan Peters \& Kathleen S. Rockland) 201-259 (Springer US, 1994).

80 Lund, J. S. Anatomical Organisation of Macaque Monkey Striate Visual Cortex. Annual review of neuroscience 11, 253-288, doi:10.1146/annurev.ne.11.030188.001345 (1988).

81 Lund, J. S. \& Yoshioka, T. Local circuit neurons of macaque monkey striate cortex: III. Neurons of laminae 4B, 4A, and 3B. Journal of Comparative Neurology 311, 234-258, doi:https://doi.org/10.1002/cne.903110206 (1991).

82 Rockland, K. S. \& Lund, J. S. Intrinsic laminar lattice connections in primate visual cortex. Journal of Comparative Neurology 216, 303-318, doi:https://doi.org/10.1002/cne.902160307 (1983).

83 Chan-Palay, V., Palay, S. L. \& Billings-Gagliardi, S. M. Meynert cells in the primate visual cortex. Journal of neurocytology 3, 631-658, doi:10.1007/BF01097628 (1974).

84 Rockland, K. S. \& Knutson, T. Axon collaterals of meynert cells diverge over large portions of area V1 in the macaque monkey. Journal of Comparative Neurology 441, 134-147, doi:https://doi.org/10.1002/cne.1402 (2001).

85 Gabbott, P. L. "Subpial Fan Cell" - A Class of Calretinin Neuron in Layer 1 of Adult Monkey Prefrontal Cortex. Frontiers in neuroanatomy 10, 28, doi:10.3389/fnana.2016.00028 (2016).

86 Nieuwenhuys, R. The neocortex. Anatomy and embryology 190, 307-337, doi:10.1007/BF00187291 (1994). 
87 Markram, $\mathrm{H}$. et al. Interneurons of the neocortical inhibitory system. Nature Reviews Neuroscience 5, 793-807, doi:10.1038/nrn1519 (2004).

88 Hahn, S. Der Leipziger Anatom Werner Spalteholz (1861-1940) und seine Beziehungen zum Deutschen Hygiene-Museum. NTM International Journal of History \& Ethics of Natural Sciences, Technology \& Medicine 7, 105-117, doi:10.1007/BF02914144 (1999).

89 Spalteholz, W. Uber das Durchsichtigmachen von menschlichen und tierischen Präparaten und seine theoretischen Bedingungen, nebst Anhang: Uber Knochenfärbung. (S. Hirzel, 1914).

90 Ulrich, N. "Vom Durchsichtig-Machen menschlicher und tierischer Gewebe“ - zwei Aufhellungspräparate nach Spalteholz aus dem Museum Anatomicum. (2017).

91 Richardson, D. S. \& Lichtman, J. W. Clarifying Tissue Clearing. Cell 162, 246-257, doi:10.1016/j.cell.2015.06.067 (2015).

92 Silvestri, L., Costantini, I., Sacconi, L. \& Pavone, F. S. Clearing of fixed tissue: a review from a microscopist's perspective. Journal of biomedical optics 21, 081205, doi:10.1117/1.JBO.21.8.081205 (2016).

93 Ueda, H. R. et al. Tissue clearing and its applications in neuroscience. Nature reviews. Neuroscience 21, 61-79, doi:10.1038/s41583-019-02501 (2020).

94 Becker, K. et al. Reduction of photo bleaching and long term archiving of chemically cleared GFP-expressing mouse brains. PloS one 9, e114149, doi:10.1371/journal.pone.0114149 (2014).

95 Belle, M. et al. Tridimensional Visualisation and Analysis of Early Human Development. Cell 169, 161-173 e112, doi:10.1016/j.cell.2017.03.008 (2017).

96 Erturk, A. et al. Three-dimensional imaging of solvent-cleared organs using 3DISCO. Nature protocols 7, 1983-1995, doi:10.1038/nprot.2012.119 (2012).

97 Renier, N. et al. Mapping of Brain Activity by Automated Volume Analysis of Immediate Early Genes. Cell 165, 1789-1802, doi:10.1016/j.cell.2016.05.007 (2016). 
98 Renier, N. et al. iDISCO: a simple, rapid method to immunolabel large tissue samples for volume imaging. Cell 159, 896-910, doi:10.1016/j.cell.2014.10.010 (2014).

99 Schwarz, M. K. et al. Fluorescent-protein stabilization and high-resolution imaging of cleared, intact mouse brains. PloS one 10, e0124650, doi:10.1371/journal.pone.0124650 (2015).

100 Chung, K. et al. Structural and molecular interrogation of intact biological systems. Nature 497, 332-337, doi:10.1038/nature12107 (2013).

101 Hama, H. et al. ScaleS: an optical clearing palette for biological imaging. Nature neuroscience 18, 1518-1529, doi:10.1038/nn.4107 (2015).

102 Hama, H. et al. Scale: a chemical approach for fluorescence imaging and reconstruction of transparent mouse brain. Nature neuroscience 14, 14811488, doi:10.1038/nn.2928 (2011).

103 Hou, B. et al. Scalable and Dil-compatible optical clearance of the mammalian brain. Frontiers in neuroanatomy 9, 19, doi:10.3389/fnana.2015.00019 (2015).

104 Ke, M. T., Fujimoto, S. \& Imai, T. SeeDB: a simple and morphologypreserving optical clearing agent for neuronal circuit reconstruction. Nature neuroscience 16, 1154-1161, doi:10.1038/nn.3447 (2013).

105 Susaki, E. A. et al. Whole-brain imaging with single-cell resolution using chemical cocktails and computational analysis. Cell 157, 726-739, doi:10.1016/j.cell.2014.03.042 (2014).

106 Dent, J. A., Polson, A. G. \& Klymkowsky, M. W. A whole-mount immunocytochemical analysis of the expression of the intermediate filament protein vimentin in Xenopus. Development 105, 61-74, doi:10.1242/dev.105.1.61 (1989).

107 Dodt, H. U. et al. Ultramicroscopy: three-dimensional visualisation of neuronal networks in the whole mouse brain. Nature methods 4, 331-336, doi:10.1038/nmeth1036 (2007).

108 Erturk, A. \& Bradke, F. High-resolution imaging of entire organs by 3dimensional imaging of solvent cleared organs (3DISCO). Experimental neurology 242, 57-64, doi:10.1016/j.expneurol.2012.10.018 (2013). 
109 Hahn, C. et al. High-resolution imaging of fluorescent whole mouse brains using stabilised organic media (sDISCO). Journal of biophotonics 12, e201800368, doi:https://doi.org/10.1002/jbio.201800368 (2019).

110 Pan, C. et al. Shrinkage-mediated imaging of entire organs and organisms using uDISCO. Nature methods 13, 859-867, doi:10.1038/nmeth.3964 (2016).

111 Pan, C. et al. Deep Learning Reveals Cancer Metastasis and Therapeutic Antibody Targeting in the Entire Body. Cell 179, 1661-1676.e1619, doi:https://doi.org/10.1016/j.cell.2019.11.013 (2019).

112 Qi, Y. et al. FDISCO: Advanced solvent-based clearing method for imaging whole organs. Science advances 5, eaau8355, doi:10.1126/sciadv.aau8355 (2019).

113 Sabdyusheva Litschauer, I. et al. 3D histopathology of human tumours by fast clearing and ultramicroscopy. Scientific reports 10, 17619, doi:10.1038/s41598-020-71737-w (2020).

114 Zhao, S. et al. Cellular and Molecular Probing of Intact Human Organs. Cell 180, 796-812 e719, doi:10.1016/j.cell.2020.01.030 (2020).

115 Žygelytè, E. et al. RetroDISCO: Clearing technique to improve quantification of retrograde labeled motor neurons of intact mouse spinal cords. Journal of neuroscience methods 271, 34-42, doi:https://doi.org/10.1016/j.jneumeth.2016.05.017 (2016).

116 Cai, R. et al. Panoptic imaging of transparent mice reveals whole-body neuronal projections and skull-meninges connections. Nature neuroscience 22, 317-327, doi:10.1038/s41593-018-0301-3 (2019).

117 Liebmann, T. et al. Three-Dimensional Study of Alzheimer's Disease Hallmarks Using the iDISCO Clearing Method. Cell reports 16, 1138-1152, doi:10.1016/j.celrep.2016.06.060 (2016).

118 Pende, M. et al. A versatile depigmentation, clearing, and labeling method for exploring nervous system diversity. Science advances 6, eaba0365, doi:10.1126/sciadv.aba0365 (2020).

119 Taylor, W. R. An enzyme method of clearing and staining small vertebrates. Proceedings of the United States National Museum (1967). 
120 Dingerkus, G. \& Uhler, L. D. Enzyme clearing of alcian blue stained whole small vertebrates for demonstration of cartilage. Stain technology 52, 229232, doi:10.3109/10520297709116780 (1977).

121 Ke, M. T. \& Imai, T. Optical clearing of fixed brain samples using SeeDB. Current protocols in neuroscience 66, Unit 222 , doi:10.1002/0471142301.ns0222s66 (2014).

122 Ke, M. T. et al. Super-Resolution Mapping of Neuronal Circuitry With an Index-Optimized Clearing Agent. Cell reports 14, 2718-2732, doi:10.1016/j.celrep.2016.02.057 (2016).

123 Tsai, P. S. et al. Correlations of Neuronal and Microvascular Densities in Murine Cortex Revealed by Direct Counting and Colocalization of Nuclei and Vessels. The Journal of Neuroscience 29, 14553, doi:10.1523/JNEUROSCI.3287-09.2009 (2009).

124 Kuwajima, T. et al. ClearT: a detergent- and solvent-free clearing method for neuronal and non-neuronal tissue. Development 140, 1364-1368, doi:10.1242/dev.091844 (2013).

125 Lai, H. M. et al. Next generation histology methods for three-dimensional imaging of fresh and archival human brain tissues. Nature communications 9, 1066, doi:10.1038/s41467-018-03359-w (2018).

126 Chen, L. et al. UbasM: An effective balanced optical clearing method for intact biomedical imaging. Scientific reports 7, 12218, doi:10.1038/s41598-017-12484-3 (2017).

127 Susaki, E. A. et al. Advanced CUBIC protocols for whole-brain and wholebody clearing and imaging. Nature protocols 10, 1709-1727, doi:10.1038/nprot.2015.085 (2015).

128 Tainaka, K. et al. Chemical Landscape for Tissue Clearing Based on Hydrophilic Reagents. Cell reports 24, 2196-2210 e2199, doi:10.1016/j.celrep.2018.07.056 (2018).

$129 \mathrm{Yu}$, T. et al. Elevated-temperature-induced acceleration of PACT clearing process of mouse brain tissue. Scientific reports 7, 38848, doi:10.1038/srep38848 (2017). 
130 Murray, E. et al. Simple, Scalable Proteomic Imaging for High-Dimensional Profiling of Intact Systems. Cell 163, 1500-1514, doi:10.1016/j.cell.2015.11.025 (2015).

131 Nojima, S. et al. CUBIC pathology: three-dimensional imaging for pathological diagnosis. Scientific reports 7, 9269, doi:10.1038/s41598017-09117-0 (2017).

132 Tainaka, K. et al. Whole-body imaging with single-cell resolution by tissue decolourization. Cell 159, 911-924, doi:10.1016/j.cell.2014.10.034 (2014).

133 Lee, E. et al. ACT-PRESTO: Rapid and consistent tissue clearing and labeling method for 3-dimensional (3D) imaging. Scientific reports 6, 18631, doi:10.1038/srep18631 (2016).

134 Liu, A. K. et al. Bringing CLARITY to the human brain: visualisation of Lewy pathology in three dimensions. Neuropathology and applied neurobiology 42, 573-587, doi:10.1111/nan.12293 (2016).

135 Morawski, M. et al. Developing 3D microscopy with CLARITY on human brain tissue: Towards a tool for informing and validating MRI-based histology. Neuroimage 182, 417-428, doi:10.1016/j.neuroimage.2017.11.060 (2018).

136 Park, Y. G. et al. Protection of tissue physicochemical properties using polyfunctional crosslinkers. Nature biotechnology, doi:10.1038/nbt.4281 (2018).

137 Phillips, J. et al. Development of passive CLARITY and immunofluorescent labelling of multiple proteins in human cerebellum: understanding mechanisms of neurodegeneration in mitochondrial disease. Scientific reports 6, 26013, doi:10.1038/srep26013 (2016).

138 Tomer, R. \& Deisseroth, K. Rapid High-resolution Brain Mapping with CLARITY Optimized Light-sheet Microscopy (COLM). Microscopy and Microanalysis 21, 717-718, doi:10.1017/s1431927615004389 (2015).

139 Tomer, R., Ye, L., Hsueh, B. \& Deisseroth, K. Advanced CLARITY for rapid and high-resolution imaging of intact tissues. Nature protocols 9, 16821697, doi:10.1038/nprot.2014.123 (2014). 
140 Treweek, J. B. et al. Whole-body tissue stabilization and selective extractions via tissue-hydrogel hybrids for high-resolution intact circuit mapping and phenotyping. Nature protocols 10, 1860-1896, doi:10.1038/nprot.2015.122 (2015).

141 Costantini, I. et al. A versatile clearing agent for multi-modal brain imaging. Scientific reports 5, 9808, doi:10.1038/srep09808 (2015).

142 Chen, F., Tillberg, P. W. \& Boyden, E. S. Expansion microscopy. Science 347, 543, doi:10.1126/science.1260088 (2015).

143 Gao, R. et al. Cortical column and whole-brain imaging with molecular contrast and nanoscale resolution. Science 363 , doi:10.1126/science.aau8302 (2019).

$144 \mathrm{Ku}$, T. et al. Multiplexed and scalable super-resolution imaging of threedimensional protein localization in size-adjustable tissues. Nature biotechnology 34, 973-981, doi:10.1038/nbt.3641 (2016).

145 Murakami, T. C. et al. A three-dimensional single-cell-resolution wholebrain atlas using CUBIC-X expansion microscopy and tissue clearing. Nature neuroscience 21, 625-637, doi:10.1038/s41593-018-0109-1 (2018).

146 Tillberg, P. W. et al. Protein-retention expansion microscopy of cells and tissues labeled using standard fluorescent proteins and antibodies. Nature biotechnology 34, 987-992, doi:10.1038/nbt.3625 (2016).

147 Lesage, M. et al. C-ECi: a CUBIC-ECi combined clearing method for threedimensional follicular content analysis in the fish ovaryt. Biology of Reproduction 103, 1099-1109, doi:10.1093/biolre/ioaa142 (2020).

148 Liu, A. K. L., Lai, H. M., Chang, R. C. C. \& Gentleman, S. M. Free of acrylamide sodium dodecyl sulphate (SDS)-based tissue clearing (FASTClear): a novel protocol of tissue clearing for three-dimensional visualisation of human brain tissues. Neuropathology and applied neurobiology 43, 346-351, doi:https://doi.org/10.1111/nan.12361 (2017).

149 Inoue, M., Saito, R., Kakita, A. \& Tainaka, K. Rapid chemical clearing of white matter in the post-mortem human brain by 1,2-hexanediol 
delipidation. Bioorganic \& medicinal chemistry letters 29, 1886-1890, doi:10.1016/j.bmcl.2019.05.049 (2019).

150 Kiernan, J. A. Histological and Histochemical Methods: Theory and Practice. (Scion, 2015).

151 Mulisch, M. \& Welsch, U. Romeis - Mikroskopische Technik. (Springer Berlin Heidelberg, 2015).

152 Li, J., Czajkowsky, D. M., Li, X. \& Shao, Z. Fast immuno-labeling by electrophoretically driven infiltration for intact tissue imaging. Scientific reports 5, 10640, doi:10.1038/srep10640 (2015).

153 Kim, S.-Y. et al. Stochastic electrotransport selectively enhances the transport of highly electromobile molecules. Proceedings of the National Academy of Sciences 112, E6274, doi:10.1073/pnas.1510133112 (2015).

$154 \mathrm{Ku}$, T. et al. Elasticizing tissues for reversible shape transformation and accelerated molecular labeling. Nature methods 17, 609-613, doi:10.1038/s41592-020-0823-y (2020).

155 Susaki, E. A. et al. Versatile whole-organ/body staining and imaging based on electrolyte-gel properties of biological tissues. Nature communications 11, 1982, doi:10.1038/s41467-020-15906-5 (2020).

156 Yun, D. H. et al. Ultrafast immunostaining of organ-scale tissues for scalable proteomic phenotyping. bioRxiv, 660373, doi:10.1101/660373 (2019).

157 Riva, M. A., Manzoni, M., Isimbaldi, G., Cesana, G. \& Pagni, F. Histochemistry: historical development and current use in pathology. Biotechnic \& Histochemistry 89, 81-90, doi:10.3109/10520295.2013.822559 (2014).

158 Kim, S. K. \& Nordén, B. Methyl green A DNA major-groove binding drug. FEBS Letters 315, 61-64, doi:https://doi.org/10.1016/00145793(93)81133-K (1993).

159 Prentø, P. Van Gieson's picrofuchsin. The staining mechanisms for collagen and cytoplasm, and an examination of the dye diffusion rate model of differential staining. Histochemistry 99, 163-174 (1993). 
160 Prentø, P. A contribution to the theory of biological staining based on the principles for structural organisation of biological macromolecules. Biotechnic \& Histochemistry 76, 137-161, doi:10.1080/bih.76.3.137.161 (2009).

161 Prentø, P. \& Lyon, H. Methyl green-pyronin Y staining of nucleic acids: studies on the effects of staining time, dye composition and diffusion rates. Biotechnic \& Histochemistry 78, 27-33, doi:10.1080/10520290312120006 (2003).

162 Santi, P. A. Light-sheet fluorescence microscopy: a review. The journal of histochemistry and cytochemistry : official journal of the Histochemistry Society 59, 129-138, doi:10.1369/0022155410394857 (2011).

163 Siedentopf, H. \& Zsigmondy, R. Ueber Sichtbarmachung ultramikroskopischer Teilchen, mit besonderer Anwendung auf Goldrubingläser. Drude's Annalen der Physik 10, 1-39 (1903).

164 Voie, A. H., Burns, D. H. \& Spelman, F. A. Orthogonal-plane fluorescence optical sectioning: Three-dimensional imaging of macroscopic biological specimens. Journal of Microscopy 170, 229-236, doi:https://doi.org/10.1111/j.1365-2818.1993.tb03346.x (1993).

165 Huisken, J., Swoger, J., Del Bene, F., Wittbrodt, J. \& Stelzer, E. H. K. Optical Sectioning Deep Inside Live Embryos by Selective Plane Illumination Microscopy. Science 305, 1007, doi:10.1126/science.1100035 (2004).

166 Keller, P. J., Pampaloni, F. \& Stelzer, E. H. K. Three-dimensional preparation and imaging reveal intrinsic microtubule properties. Nature methods 4, 843-846, doi:10.1038/nmeth1087 (2007).

167 Keller, P. J. \& Stelzer, E. H. K. Quantitative in vivo imaging of entire embryos with Digital Scanned Laser Light-sheet Fluorescence Microscopy. Current opinion in neurobiology 18, 624-632, doi:https://doi.org/10.1016/j.conb.2009.03.008 (2008).

168 Reynaud, E. G., Peychl, J., Huisken, J. \& Tomancak, P. Guide to light-sheet microscopy for adventurous biologists. Nature methods 12, 30-34, doi:10.1038/nmeth.3222 (2015). 
169 Reynaud, E. G., Krzic, U., Greger, K. \& Stelzer, E. H. Light-sheet-based fluorescence microscopy: more dimensions, more photons, and less photodamage. HFSP journal 2, 266-275, doi:10.2976/1.2974980 (2008).

170 Tomer, R. et al. SPED Light-sheet Microscopy: Fast Mapping of Biological System Structure and Function. Cell 163, 1796-1806, doi:10.1016/j.cell.2015.11.061 (2015). 
Chapter 1 - General Introduction 
CHAPTER 2

\section{Scalable Labelling for Cytoarchitectonic}

\section{Characterisation of Large Optically \\ Cleared Human Neocortex Samples}

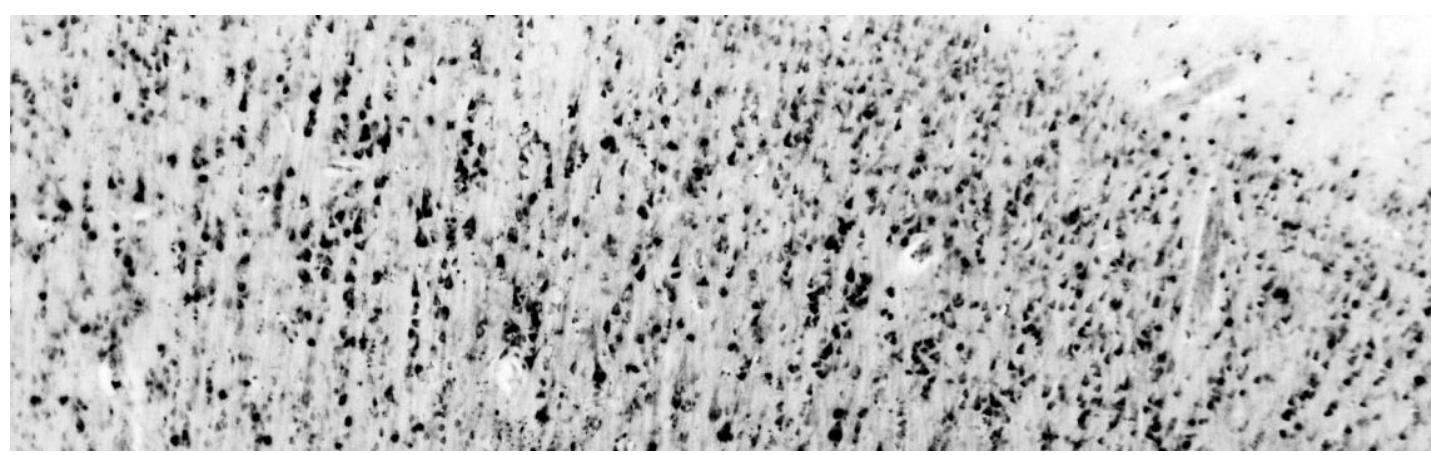

Supragranular layers of human $\mathrm{V} 2$ revealed by MASH.

Sci Rep 9, 10880 (2019).

https://doi.org/10.1038/s41598-019-47336-9

PMID: 31350519

Sven Hildebrand, Anna Schueth, Andreas Herrler, Ralf Galuske \& Alard Roebroeck 


\section{Abstract}

Optical clearing techniques and light-sheet microscopy have transformed fluorescent imaging of rodent brains and have provided a crucial alternative to traditional confocal or bright field techniques for thin sections. However, clearing and labelling human brain tissue through all cortical layers and significant portions of a cortical area, has so far remained extremely challenging, especially for formalin fixed adult cortical tissue. Here, we present MASH (Multiscale Architectonic Staining of Human cortex): a simple, fast and low-cost cytoarchitectonic labelling approach for optically cleared human cortex samples, which can be applied to large (up to $5 \mathrm{~mm}$ thick) formalin-fixed adult brain samples. A suite of small-molecule fluorescent nuclear and cytoplasmic dye protocols in combination with new refractive index matching solutions allows deep volume imaging. This greatly reduces time and cost of imaging cytoarchitecture in thick samples and enables classification of cytoarchitectonic layers over the full cortical depth. We demonstrate application of MASH to large archival samples of human visual areas, characterizing cortical architecture in 3D from the scale of cortical areas to that of single cells. In combination with scalable light-sheet imaging and data analysis, MASH could open the door to investigation of large human cortical systems at cellular resolution and in the context of their complex 3-dimensional geometry. 


\section{Introduction}

Cellular resolution 3D volume microscopy of human cerebral cortex is challenging because of the large size of the human brain and the 3dimensional geometry of the cortex. The 2-4 mm thick cortical sheet is highly curved and packed with billions of neurons, organized in layers each hundreds of micrometres thick. Traditionally, studies on human cortical cytoarchitecture have been performed on sections with a thickness of less than $100 \mu \mathrm{m}$. However, thin sections have no clear geometric relation to the curved cortical sheet, mostly slicing it non-orthogonal to the layer organisation ${ }^{1}$. Moreover, due to shape distortions and tearing inherent to the sectioning process, serial sections are extremely difficult to align post-hoc to provide valuable 3D maps of cytoarchitecture. Although a recent surge of optical clearing techniques has transformed microscopic 3D imaging of small transgenic or antibody-stained rodent brains ${ }^{2-7}$, translation of these techniques to the much larger adult human brain has remained a challenge. More specifically, labelling for cytoarchitectonic characterisation of adult formalin fixed brain samples has so far remained out of reach, particularly when it needs to be scalable in terms of time and cost to the scale of human neocortical areas.

Here, we report MASH (Multiscale Architectonic Staining of Human cortex): a novel scalable nuclear and cytoplasmic labelling approach for optically cleared samples. MASH is suitable for 4-5 mm thick archival (i.e. formalin fixed and long-term stored) adult human cortex 
samples and enables deep 3D optical imaging. MASH consists of two parts: 1) a set of small-molecule fluorescent dyes and cleared tissue cytoarchitecture labelling protocols (MASH dye protocols) and 2) a set of adjustable refractive index-matching solutions (MASH RIMS) which provide a less corrosive alternative to commonly used RIMS in solvent-based clearing protocols. For the MASH dye protocols, we identified four small organic compounds: acridine orange (AO), methylene blue (MB), methyl green (MG) and neutral red (NR), previously used as cytoplasmic and nuclear labels in traditional light microscopy studies ${ }^{8,9}$. We developed adapted protocols for their use as fluorescent labels in large cleared human brain specimen: MASHAO (green spectrum cell body label), MASH-NR (red spectrum cell body label), MASH-MB (far-red spectrum cell body label) and MASHMG (far-red spectrum cell nucleus label).

In order to apply the MASH dye protocols in a wide range of human cortex samples, the clearing process must be: 1) potent enough to clear highly myelinated adult human brain tissue up to 4-5 $\mathrm{mm}$ thickness within reasonably short time, 2) compatible with MASH dye protocols and, 3) applicable to archival samples available from brain banks and other academic and clinical tissue storing facilities. The DISCO family of solvent-based clearing protocols ${ }^{4,6,10}$ have short clearing times and they have been applied to freshly frozen ${ }^{11}$ and formalin fixed ${ }^{10}$ human brain tissue. A minor challenge in these protocols is posed by the refractive index-matching solutions (RIMS): dibenzyl ether (DBE) or mixtures of benzyl alcohol (BA), benzyl 
benzoate (BB) and diphenyl ether (DPE). Their corrosiveness limits the microscope setups, which can be used due to the detrimental effects on many microscope objectives. Therefore, we developed an adapted DISCO approach replacing the RIMS with two new MASH RIMS alternatives. We identified the essential oil trans-cinnamaldehyde (CA) as a high refractive index (RI) medium, mixable with 2,2'Thiodiethanol (TDE) or wintergreen oil (WGO) to create TDE/CA and WGO/CA RIMS. TDE has been used in the past in aqueous, buffered solution for RI-matching of CLARITY processed tissue (RI=1.46), showing compatibility with immunostainings and transgenic labels ${ }^{12}$. The MASH RIMS have the desirable properties of adjustable RI (from 1.52 to 1.62 , therefore compatible to the DISCO RI of 1.56), low melting point and low corrosiveness, which make them a suitable alternative RIMS choice. Applied together, the MASH dye protocols and MASH RIMS allow clearing and labelling of thick adult human brain samples and deep volume imaging of cytoarchitecture.

We first describe the MASH protocol for clearing and labelling thick human formalin fixed brain samples. Subsequently, we show that the four MASH dyes are suitable for both point scanned and light-sheet fluorescent 3D microscopy in different colour bands, and that signal is well maintained even when imaging very deep into the cleared tissue. To validate MASH as a cytoarchitecture technique, we compare fluorescent MASH labels against more traditional bright-field Nissl stains on thin sections, and show that known cytoarchitectonic layer classifications can be performed in 3D MASH datasets. Finally, we 
demonstrate on large archival samples of human visual areas that MASH has the potential to characterise cortical architecture in 3D from the scale of cortical areas to that of single cells when combined with light-sheet fluorescence microscopy. 


\section{Materials \& Methods}

\section{Human brain tissue}

Brain tissue samples were taken from 3 different human body donors (no known neuropathological diseases) of the body donation program of the Department of Anatomy and Embryology, Maastricht University. The tissue donors gave their informed and written consent to the donation of their body for teaching and research purposes as regulated by the Dutch law for the use of human remains for scientific research and education ("Wet op de Lijkbezorging"). Accordingly, a handwritten and signed codicil from the donor posed when still alive and well, is kept at the Department of Anatomy and Embryology Faculty of Health, Medicine and Life Sciences, Maastricht University, Maastricht, The Netherlands.

Brains were first fixed in situ by full body perfusion via the femoral artery. Under a pressure of 0.2 bar the body was perfused by 101 fixation fluid (1.8 vol \% formaldehyde, $20 \%$ ethanol, $8.4 \%$ glycerine in water) within 1.5-2 hours. Thereafter, the body was preserved at least 4 weeks for post-fixation submersed in the same fluid. Subsequently, brains were recovered by calvarial dissection and stored in $4 \%$ paraformaldehyde in $0.1 \mathrm{M}$ phosphate buffered saline (PBS) for 14-30 months.

For MASH clearing and whole-mount labelling procedures, tissue of an occipital lobe (subject 1, fixation time 14 months), occipital and 
parietal samples (subject 2, fixation time 30 months) and a temporal lobe sample (subject 3 , fixation time 3 months) were used (Supplementary Table 1). All tissue was manually blocked with anatomical trimming blades, then cut into 2 to $5 \mathrm{~mm}$ thick slices in coronal orientation and immediately processed.

All methods were carried out in accordance with the relevant guidelines and regulations and all experimental protocols were approved by the Ethics Review Committee Psychology and Neuroscience (ERCPN).

\section{MASH protocol for clearing and labelling of human brain samples}

For clearing of formalin-fixed adult brain tissue an adaptation of the iDISCO + method $^{13}$ was used. The incubation times were adjusted for better clearing of formalin-fixed adult human brain tissue and dibenzyl ether (DBE) was replaced by two new MASH refractive index matching solutions (RIMS) adjusted to an RI of 1.56, as described below. The MASH protocol consists of the following steps: 1) sample pre-treatment with methanol and bleaching, 2) labelling with MASH dye protocols and 3) clearing and refractive index matching with MASH RIMS.

\section{Sample pre-treatment with methanol and bleaching}

For the pre-treatment, samples were dehydrated in ascending concentrations of methanol (VWR International, LLC, 20846.326) in distilled water: $1 \mathrm{~h}$ each in $20 \%, 40 \%, 60 \%, 80 \%$, and twice in $100 \%$. 
Samples were cooled down to $4{ }^{\circ} \mathrm{C}$ during the second incubation in $100 \%$ methanol. This was followed by bleaching with freshly prepared $5 \% \mathrm{H}_{2} \mathrm{O}_{2}$ (1 volume of $30 \% \mathrm{H}_{2} \mathrm{O}_{2}$ for 5 volumes of methanol, ice cold) at $4{ }^{\circ} \mathrm{C}$ overnight under shaking. After bleaching and re-equilibration to room temperature (RT) the tissue was rehydrated as follows: incubation for $1 \mathrm{~h}$ in $80 \%, 60 \%, 40 \%$ and $20 \%$ methanol in distilled water and twice in in $0.1 \mathrm{M}$ PBS/0.2 \% Triton X-100 (VWR International, LLC, 28817.295) respectively. After this, labelling was performed as described below. For all steps, incubation was done in 6 well cell culture plates (Corning Inc., 3516) in a volume of $5 \mathrm{ml} /$ well at RT, unless mentioned otherwise.

\section{Labelling procedure with MASH dye protocols}

Four dyes were identified for cell body (cytoplasm and nucleus) staining or nuclear staining. These dyes, used variously before in animal studies as bright-field or fluorescent stain on histological sections, were investigated as to their suitability as labels of large, cleared adult human specimen for deep fluorescent light microscopy imaging. The four dyes acridine orange (AO), methylene blue (MB), methyl green (MG) and neutral red (NR), see Supplementary Table 4, are referred to as MASH-AO, MASH-MB, MASH-MG and MASH-NR respectively in the context of human cleared tissue labelling protocols, to distinguish them clearly from other prior uses. All MASH labels are small organic compounds with low molecular weight and fluorescent properties which have hitherto not been applied for 
labelling in thick, cleared human tissue. $\mathrm{AO}$ and $\mathrm{NR}$ have been variously described before as bright-field or fluorescent Nissl stains on standard histological sections ${ }^{9}$. MG was recently described as an effective, low-cost DNA stain, and applied to chick embryo cryosections and whole-mount zebrafish embryos ${ }^{8}$. MB has been used since the early $20^{\text {th }}$ century as a bright-field Nissl stain on thin sections. Here, we optimized the staining protocols for use with thick optically cleared human tissue samples requiring orders of magnitude lower concentrations than bright-field application.

The applied optimized labelling protocol was as follows: all samples were first incubated in freshly filtered solution of $50 \%$ potassium disulfite (Sigma-Aldrich, 55777) in distilled water for $1 \mathrm{~h}$ at RT. The samples were then washed for $1 \mathrm{~h}$ at RT in distilled water. MG stock solution was prepared according to the method of Prieto et al. ${ }^{8}$. A $4 \%$ aqueous MG (Sigma-Aldrich, 67060) solution was prepared, and crystal violet impurities were removed by extractions with chloroform (Sigma-Aldrich, 372978), discarding the lower (violet) phase until no violet tinge could be observed in the lower phase. Stock solution was diluted 1:5000 in 0.1 M PBS with $1 \%$ sodium azide (Sigma-Aldrich, S2002) at $\mathrm{pH}$ 7.4. Staining procedure for $\mathrm{AO}$ and NR was based on the protocols described in Schmued et al. ${ }^{9}$. For AO (CarlRoth, 7632.2) and NR (CarlRoth, T122.1) staining, a $1 \%$ stock solution in $0.1 \mathrm{M}$ PBS with $1 \%$ sodium azide at $\mathrm{pH} 4$ was prepared for each dye. $1 \% \mathrm{MB}$ (CarlRoth, A514.1) stock solution was prepared in PBS with $1 \%$ sodium azide at $\mathrm{pH}$ 7.4. Samples for AO, NR and MB staining were incubated in a final 
concentration of $0.001 \%$ in $0.1 \mathrm{M} \mathrm{PBS}$ with $1 \%$ sodium azide at either $\mathrm{pH} 4(\mathrm{AO}, \mathrm{NR})$ or $\mathrm{pH} 7.4$ (MB) for 1 day/mm tissue thickness (2-5 days total) at $4{ }^{\circ} \mathrm{C}$. All staining steps were carried out again in 6 well-plates in a volume of $6 \mathrm{ml} /$ well on a shaker.

\section{Clearing and refractive index matching with MASH-RIMS}

After labelling, samples were washed twice for $1 \mathrm{~h}$ in $0.1 \mathrm{M}$ PBS of the respective $\mathrm{pH}$ and dehydrated in ascending concentration of methanol in water: $1 \mathrm{~h}$ each in $20 \%, 40 \%, 60 \%, 80 \%$, and twice in $100 \%$. A volume of $5 \mathrm{ml}$ per sample was used for each solution and incubation was performed at RT. Given the corrosiveness of the involved solutions, samples were then transferred into $50 \mathrm{ml}$ incubation tubes made of high-density polyethylene (HDPE). Subsequently, samples were incubated for $3 \mathrm{~h}$ ( $2 \mathrm{~mm}$ thick samples) or overnight (5 mm thick samples; see Supplementary Table 1) in a mixture of $33 \%$ methanol / 66 \% dichloromethane (DCM, CarlRoth, 8424.2). Remaining methanol was washed out by incubation in $100 \%$ DCM twice for $15 \mathrm{~min}$ ( $2 \mathrm{~mm}$ thickness) or twice for $1 \mathrm{~h}(5 \mathrm{~mm}) .50 \mathrm{ml}$ tubes were filled completely with each solution.

For refractive index matching, cleared (dehydrated and delipidated) samples were incubated overnight at RT (transparency can already be achieved after several hours of incubation depending on sample thickness and the RIMS used) in $25 \mathrm{ml}$ of one of the newly proposed MASH RIMS: 1) WGO/CA: 72 \% methyl salicylate also known as wintergreen oil (WGO, Sigma-Aldrich, 84332) and $28 \%$ trans- 
Cinnamaldehyde (CA, Sigma-Aldrich, C80687), 2) TDE/CA: 62 \% 2,2Thiodiethanol (TDE, Sigma-Aldrich, 166782) and $38 \%$ CA. The RIMS was changed once right before imaging and tubes were turned upside-down several times until no streaks were visible anymore in the fluid before mounting for microscopic imaging.

\section{Counterstaining cleared and MASH labelled samples with DAPI.}

Counterstains were performed with 4',6-diamidino-2-phenylindole (DAPI) to label cell nuclei in cleared samples labelled with MASH-AO and MASH-NR. For DAPI labelling, 100 mg DAPI (CarlRoth, 6843.3) was dissolved in distilled water to prepare a $0.8 \mathrm{mg} / \mathrm{ml}$ stock solution. This stock solution was diluted 1:800 into the freshly prepared working solutions at the respective $\mathrm{pH}$, resulting in a final concentration of 1 $\mu \mathrm{g} / \mathrm{ml}$. For labelling, each brain sample was incubated in a volume of $6 \mathrm{ml}$ for 2 or $5 \mathrm{~d}$ at $4{ }^{\circ} \mathrm{C}$ on a shaker for 2 or $5 \mathrm{~mm}$ thick samples respectively. For incubation 6 well-plates were used. For DAPI colabelling of sections, an incubation time of $15 \mathrm{~min}$ at RT was used.

\section{MASH-RIMS property comparison}

For refractive index matching of dehydrated and delipidated human tissue, the properties of several substances and solutions of various RI's were evaluated for their use as RIMS (Suppl. Table 2). As a previously unexplored substance for RIMS, trans-Cinnamaldehyde (CA, Sigma-Aldrich, C80687) was identified, an essential oil with a very 
high RI of 1.62 and low melting point which is mixable with TDE and WGO.

The following mixtures were evaluated for their clearing capacity of the dehydrated and delipidated adult human brain samples: $80 \%$ glycerol in 0.1 M PBS (pH 7.4, RI = 1.44) and mineral oil (Sigma-Aldrich, M5904) with an RI of 1.47, pure TDE (Sigma-Aldrich, 166782) with an RI of 1.52, pure WGO (RI = 1.54, Sigma-Aldrich, 84332), and pure ethyl cinnamate (ECi, RI=1.56, Sigma-Aldrich, 112372). Furthermore, mixtures of either $72 \% \mathrm{WGO}$ and $28 \% \mathrm{CA}$ or $62 \% \mathrm{TDE}$ and $38 \% \mathrm{CA}$ with an RI of 1.56 respectively and a solution of $38 \%$ TDE and $62 \% \mathrm{CA}$ with an RI of approximately 1.58 were tested. As a control, $0.1 \mathrm{M}$ PBS was used. To evaluate corrosiveness, the compatibility with selected plastic materials was checked. Several containers made from polystyrene, polypropylene, high-density polyethylene and tetrafluoroethylene were incubated for up to one week with the solutions described above.

The two MASH RIMS TDE/CA (62 \% TDE and $38 \%$ CA, RI=1.56) and WGO/CA (72\% WGO and $28 \% \mathrm{CA}, \mathrm{RI}=1.56$ ) were identified as having the combined desirable properties of an ideal (and adaptable) RI, low photobleaching, low corrosiveness and convenient storage at low temperature (low melting point). These solutions render archival human brain samples highly transparent with a slight remaining amber colour (Fig. 2 b, g; Suppl. Fig. 6) typical for many solvent-based clearing approaches. The transparency achieved with the recently 
described $\mathrm{ECi}^{14}$ is similar to the WGO/CA and TDE/CA RIMS (Supplementary Figure 6). However, ECi has a melting point of $6-8{ }^{\circ} \mathrm{C}$ and samples cannot be stored in the fridge once immersed in the liquid. Overall, the TDE/CA RIMS was preferred, because it is even less corrosive for plastic equipment than WGO/CA and had better properties for long-term cold storage (low melting point) than ECi.

\section{Standard histological sectioning}

For the optimisation of the final staining protocol (described above) as well as for the validation experiments, standard histological sections of the same brain tissue were used. Therefore, manually cut blocks of human neocortical tissue were sectioned on a vibratome (VT1200 S, Leica Mikrosysteme Vertrieb GmbH, Wetzlar, Germany) into $50 \mu \mathrm{m}$ thick sections.

\section{Optimisation of staining conditions for MASH dye protocols}

Staining conditions for MASH-AO and MASH-NR, in terms of $\mathrm{pH}$ and pre-treatment were optimized for maximum contrast (Suppl. Fig. 4 and 5). Before staining, sections were incubated in either $50 \%$ freshly filtered potassium disulfite solution for $15 \mathrm{~min}$, dehydrated and delipidated in $70 \%, 100 \%$ and $70 \%$ methanol for 5 min each or treated first with potassium disulfite and then methanol. Control sections were incubated in $0.1 \mathrm{M}$ PBS for 30 min (Supplementary Figure 4 and $5)$. Sections were then washed for $5 \mathrm{~min}$ in distilled water and stained with $\mathrm{AO}$ or NR in the respective dye solutions described above for 15 
min and washed in $0.1 \mathrm{M}$ PBS of the respective $\mathrm{pH}$ for 5 min twice. Afterwards, the sections were mounted in Kaiser's glycerol gelatine (CarlRoth, 6474.1).

\section{Validation of specificity of MASH dye protocols}

Three validation experiments were performed on standard sections. 1) MASH dye protocols MASH-AO, MASH-NR and MASH-MB were compared in the same sections with standard bright-field (BF) stain Cresyl Violet. 2) MASH dye protocol MASH-MG was compared in the same sections with standard fluorescent stain DAPI. 3) MASH dye protocols MASH-NR, MASH-MB and MASH-MG were compared in the same sections with higher concentrations of the same dye imaged as a bright-field stain.

For experiment 1, standard histological sections were pretreated with potassium disulfite solution and stained with MASH dye protocols MASH-AO, MASH-NR and MASH-MB as described. Sections were then dehydrated in $50 \%, 70 \%$ and $100 \%$ methanol for 5 min each, delipidated in $66 \% \mathrm{DCM} / 33 \%$ methanol for $15 \mathrm{~min}$, and washed twice in $100 \%$ DCM for 5 min. This was followed by rehydration in $100 \%$, $70 \%, 50 \%$ methanol and twice in $0.1 \mathrm{M} \mathrm{PBS}$ of the respective $\mathrm{pH}$ for 5 min. Sections were mounted in Kaiser's glycerol gelatine. For comparison of the MASH dye protocols with bright-field stains, coverslips of the MASH-labelled sections were removed by immersion in warm water after fluorescent imaging. Subsequently, sections were washed in warm distilled water for $5 \mathrm{~min}$ and stained with cresyl violet 
acetate (AlfaAesar, J64318) by first dehydration in $70 \%$ and $100 \%$ ethanol for $5 \mathrm{~min}$ and rehydration in $70 \%$ ethanol and distilled water for $2 \mathrm{~min}$. This was followed by incubation in freshly filtered $50 \%$ aqueous potassium disulfite solution for $15 \mathrm{~min}$ and two washes in distilled water for $5 \mathrm{~min}$ each. For staining a filtered cresyl violet solution of $1.5 \%$ in water, $1 \%$ acetic acid and $1 \% 1 \mathrm{M}$ sodium acetate was used. Samples were stained for $5 \mathrm{~min}$ and subsequently washed in acetate buffer for $2 \mathrm{~min}$. Differentiation and dehydration was performed in $70 \%, 96 \%$ and $100 \%$ ethanol. Finally, samples were immersed in xylol for 5 min twice and mounted in Entellan ${ }^{\circledR}$ (Suppl. Fig. $1 \mathrm{a}-\mathrm{i})$. For experiment 2 on MASH-MG, the same procedures were followed, but sections were co-labelled with DAPI as described above for DAPI counterstaining (Suppl. Fig. $1 \mathrm{j}-1$ ).

For experiment 3, sections fluorescently labelled with MASH-MB, MASH-MG and MASH-NR were stained with higher concentrations of the same dyes as a BF control. To this end, coverslips were removed, and the sections were washed as described above. Sections were then stained in aqueous stock solutions of $\mathrm{MB}$ or $\mathrm{MG}$, or in $0.1 \%$ of $\mathrm{NR}$ in $0.1 \mathrm{M} \mathrm{PBS}$ at pH 4 for 5 min respectively. Hereafter, sections were washed 5 min in 0.1 M PBS with a pH of 7.4 (MB and MG) or pH 4 (NR) and differentiated in $70 \%$ and $100 \%$ ethanol. After two $3 \mathrm{~min}$ incubations in xylol, sections were mounted in Entellan ${ }^{\circledR}$ and imaged (Suppl. Fig. 3). 


\section{Magnetic resonance imaging}

Data acquisitions on the occipital lobe host sample were performed on a research 9.4 $\mathrm{T}$ Siemens MAGNETOM scanner (Siemens Healthcare, Erlangen, Germany) according to the methods described in $^{15}$. A 3D multi-echo Gradient echo (GRE) sequence was used to acquire $200 \mu \mathrm{m}$ isotropic data (Repetition time /Echo times: $45 \mathrm{~ms}$ / 7.86, 14, 24 and $34 \mathrm{~ms}$, flip angle $(\mathrm{FA})=28 \mathrm{deg}$, bandwidth $(\mathrm{BW})=120$ $\mathrm{Hz} / \mathrm{px}$, matrix dimensions $=400 \times 400 \times 416$ ). Quantitative $\mathrm{T}_{2}$ * estimation was performed by fitting a mono exponential decay model and a 3D surface reconstruction was created using Brain Voyager QX v2.8.

\section{Light-sheet and two-photon microscopic imaging}

Before two-photon microscopic imaging (TPM), stained and cleared human brain samples were transferred to a glass petri dish, and a cover slip with water drop was placed on top of each sample. For TPM imaging experiments, a two-photon laser scanning microscope (Leica TCS SP5 MP, Leica Mikrosysteme Vertrieb GmbH, Wetzlar, Germany), equipped with a HCX APO L 20x/1.00W water immersion objective was used. Working distance of the objective was $2 \mathrm{~mm}$ and the excitation source was a $140 \mathrm{fs}$-pulsed Ti:sapphire laser (Chameleon Ultra II, Coherent Inc., Santa Clara, CA, USA), mode-locked at $800 \mathrm{~nm}$. To avoid photobleaching and tissue damage, laser power was kept at $11 \%$ resulting in approx. $25-50 \mathrm{~mW}$, at the sample surface. Images and image stacks were acquired with Leica Application Suite Advanced 
Fluorescence (Leica Microsystems). TPM Image acquisition settings are detailed in Supplementary Table 3.

Light-sheet fluorescence microscopic (LSFM) imaging was performed with the Ultramicroscope II (La Vision Biotech, Bielefeld, Germany), equipped with a SuperK Extreme Supercontinuum white light laser (EXW-12, NKT Photonics, Birkerød, Denmark). The used objective was a MVPLAPO 2X C/0,5 NA objective with dipping cap (Olympus, Japan), a working distance of $5.7 \mathrm{~mm}$ and a RI range of 1,33-1,56. The brain samples were fully immersed during the imaging process in $160 \mathrm{ml}$ of imaging medium. TPM and LSFM acquisition settings for all experiments are detailed in Supplementary Table 3.

\section{Bright-field and fluorescence microscopy of thin tissue sections}

For all imaging experiments on (non-cleared) vibratome sections an Olympus BX51WI DSU confocal microscope (Olympus, Center Valley, PA, USA) coupled to a Hamamatsu EM-CCD C9100 camera (Hamamatsu Photonics K. K., Hamamatsu, Japan) was used. The system was equipped with a motorized stage and a LEP MAC 5000 Controller System (Ludl electronic products, Hawthorne, NY, USA). Images were taken with either an Olympus PlanApo 2x/0.08 NA, 4x/0.16 NA or UPlanSApo 10x/0.40 NA objective (Olympus, Center Valley, PA, USA). Excitation and emission characteristics for all dyes are given in Supplementary Table 2. For acquisition, the Stereo Investigator software (MBF Bioscience, Williston, Vermont, USA) was used. 


\section{Microscopy data processing}

For image post-processing such as brightness and contrast adjustments, subtraction of background, and thresholding, as well as running depth-stack volume visualisation, the open source software FIJI was used ${ }^{16}$. 3D Volume rendering was performed using Bitplane IMARIS. Quantitative signal analysis was performed in MATLAB using the Open Microscopy Environment (OME) MATLAB toolbox. For the cell body and tissue background signal analysis variation over imaging depth (Figure 2b), at each depth of $500 \mu \mathrm{m}, 1500 \mu \mathrm{m}, 2500 \mu \mathrm{m}$, $3500 \mu \mathrm{m}$ and $4500 \mu \mathrm{m}$ from the surface of the tissue, three consecutive data planes were used from the imaging stack for analysis (15 planes total). The middle $50 \%$ (in the light propagation direction) of each image plane was used to discard data away from the lightsheet waist. At each of the five depths, a total of fifteen regions-ofinterest (five per plane, three planes) of $3 \times 3$ pixels were selected for brightness assessment in both cell bodies and tissue background. The signal for each region-of-interest was taken as the average over the 3 x 3 pixel area. For both cell bodies and tissue background, boxplots were created depicting mean ('+' sign), median (box center line), 25th and 75th percentiles (box edges) and 9th and 91st percentiles (whiskers) of the $\mathrm{n}=15$ region-of-interest signals at each depth. Pairwise testing for difference in mean cell body signal at different depths $(1500 \mu \mathrm{m}$ vs. $2500 \mu \mathrm{m}, 1500 \mu \mathrm{m}$ vs. $3500 \mu \mathrm{m}$, and $1500 \mu \mathrm{m}$ vs. $4500 \mu \mathrm{m})$ and mean background signal (at the same depths) was performed by unpaired (two-sample) t-tests with un-equal variance. 
The $500 \mu \mathrm{m}$ depth plane was excluded from testing as the sample geometry caused tissue at that depth to be imaged at the furthest distance from the light-sheet excitation, experiencing greater signal decay due to scattering. Significance level was set at $\alpha=0.05$, Bonferroni-corrected over the 6 tests, i.e. $\alpha=0.0083$ for each test.

For the pixel-by-pixel overlap of CV label and MASH-NR label in a thin section (Fig. 3), as well as for $\mathrm{CV} / \mathrm{MASH}-\mathrm{AO}, \mathrm{CV} / \mathrm{MASH}-\mathrm{MB}$ and DAPI/MASH-MG overlap (Suppl. Fig. 2) the (inverted) CV and MASH dye images were smoothed (Gaussian filter with sigma $=1$ pixel) to remove noise, thresholded, and binarized, and the Phi coefficient (Pearson correlation between binary variables) and Jaccard index (size of the intersecting pixel set divided by the size of the union pixel set) were computed. To obtain pixel-by-pixel overlap scores that are relatively independent of threshold and different signal levels in the data, each of the Phi coefficients and Jaccard indices were computed for a range of thresholds and the maximum value achieved with a single fixed threshold $t_{1}$ for gold standard image (CV or DAPI) and $t_{2}$ for MASH dye (NR, $\mathrm{AO}, \mathrm{MB}$ or $\mathrm{MG}$ ) is reported. 


\section{Results}

We demonstrated that MASH can clear and label archival adult cortex samples and that cytoarchitecture can be imaged at a variety of wavelengths and magnifications (Fig. 1). Volume imaging can be performed in the green spectrum (MASH-AO, Fig. 1 a, b, d; Suppl. Video 1), red spectrum (MASH-NR, Fig. 1 c, Fig. 2) and far-red spectrum (MASH-MB, MASH-MG, Fig. 1 e, f; Suppl. Video 5). Green spectrum MASH-AO and red-spectrum MASH-NR are best suited for imaging at sub-micron resolutions using two-photon microscopy (TPM), because their wavelength allows for two-photon excitation by Ti:Sapphire lasers and for relatively high diffraction-limited resolution. This allows delineating single neuron cell body morphology (Fig. 1 a), high resolution reconstruction in 3d (Fig. 1 b) and delineation of cortical layer borders (Fig. 1 c, d). Two-photon excitation of far-red spectrum MASH-MB and MASH-MG lies outside the range of Ti:Sapphire lasers and their longer wavelengths permit lower diffraction limited resolutions. Instead, MASH-MB and MASHMG, as well as red spectrum MASH-NR, are well suited for very deep and large field of view imaging, because of the lower scattering at red and far-red wavelengths. Therefore, imaging with light-sheet fluorescence microscopy (LSFM; Fig. 1 e, f) can be performed over larger fields of view up to the entire cortical sheet. 


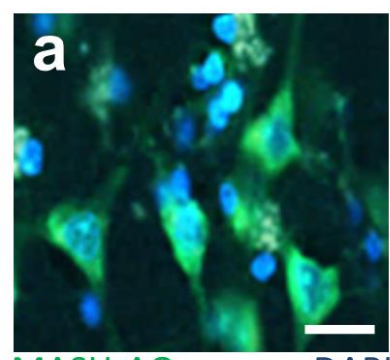

MASH-AO

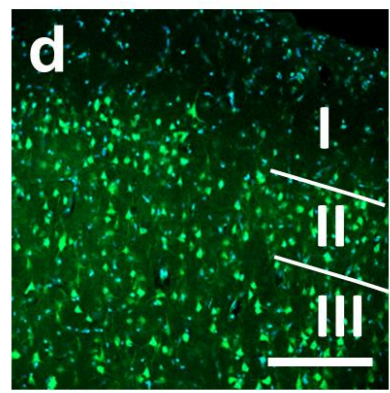

MASH-AO

DAPI

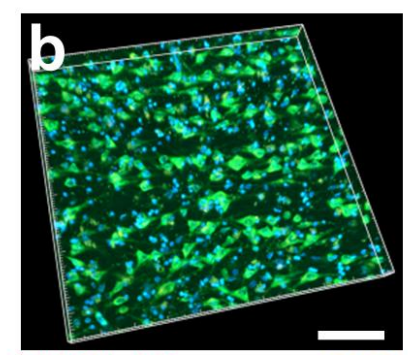

MASH-AO

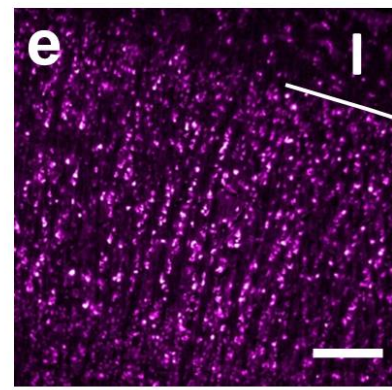

MASH-MB
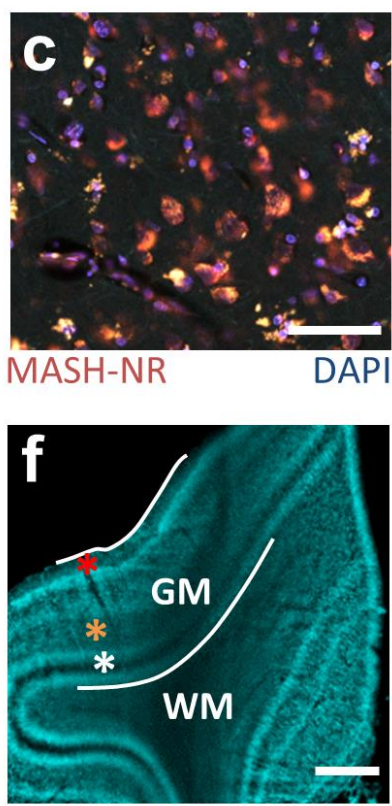

MASH-MG

Figure 1: MASH labels human cortical cytoarchitecture in cleared formalin-fixed tissue imaged at high resolution and depth. (a) Two-photon microscopy (TPM) image of a cleared human neocortex sample stained with MASH-AO for neuronal cell bodies (green), counterstained with DAPI (blue) for cell-nuclei. (b) 3D rendering of the imaging stack in a. (c) TPM image of a cleared sample stained with MASHNR for neuronal somata (red) and DAPI (blue). (d) TPM image of MASH-AO and DAPI stain showing the layer I/II and II/III borders. (e) Light-sheet fluorescence microscopy (LSFM) of MASH-MB showing the layer I/II border. (f) Lowmagnification LSFM imaging of MASH-MG stain showing the entire gray matter (GM), the white matter (WM) transition and cortical layer contrast (red asterisk: layer I; orange asterisk: stripe of Gennari, layer IVb; white asterisk: inner stripe of Baillarger, layer V). Scale bars a: $20 \mu \mathrm{m}$; b, c: $50 \mu \mathrm{m}$; d: $100 \mu \mathrm{m}$; e: $200 \mu \mathrm{m}$; f: 1 mm. 


\section{Depth of imaging}

Clearing and staining can be performed on $5 \mathrm{~mm}$ thick samples in a matter of 10 days. The tissue clearing steps, similar to iDISCO+, led to a slight tissue shrinkage of $\sim 10 \%$ bringing a $5 \mathrm{~mm}$ pre-clearing tissue sample to about $4.5 \mathrm{~mm}$ post-clearing. Clearing and staining result in imaging of cell bodies with low background and high signal over the full imaging depth (Fig. 2). With the red spectrum MASH-NR dye, neural cell body signal shows only a modest decrease in going from imaging at the first $500-1500 \mu \mathrm{m}$ of depth to $4500 \mu \mathrm{m}$ of imaging depth from the surface (Fig. 2 b, c-g), whereas tissue background signal stays roughly the same. Unpaired t-tests for difference of mean signal (p-values $1500 \mu \mathrm{m}-2500 \mu \mathrm{m}$ : p=0.453; $1500 \mu \mathrm{m}-3500 \mu \mathrm{m}$ : $\mathrm{p}=0.453 ; 1500 \mu \mathrm{m}-4500 \mu \mathrm{m}: \mathrm{p}=0.0102)$ and difference of mean background (p-values $1500 \mu \mathrm{m}-2500 \mu \mathrm{m}$ : p=0.932; 1500 $\mu \mathrm{m}-3500 \mu \mathrm{m}$ : $\mathrm{p}=0.447 ; 1500 \mu \mathrm{m}-4500 \mu \mathrm{m}: \mathrm{p}=0.565)$ did not reach significance at a Bonferroni corrected $\alpha=0.05$. Note that the colour scale in figure $2 \mathrm{c}-$ $\mathrm{g}$ was chosen to illustrate signal and tissue background levels, rather than suppressing background and emphasizing cell body signal. 


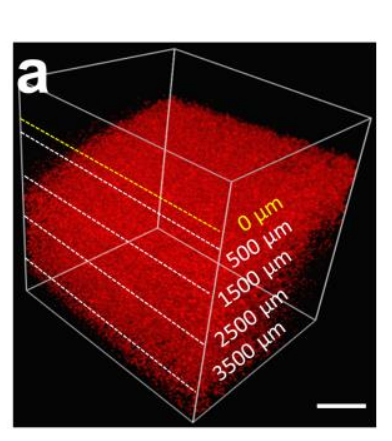

MASH-NR

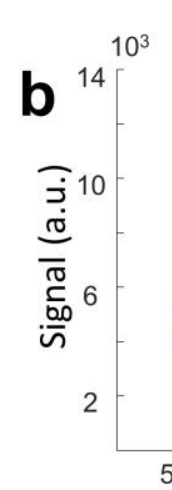

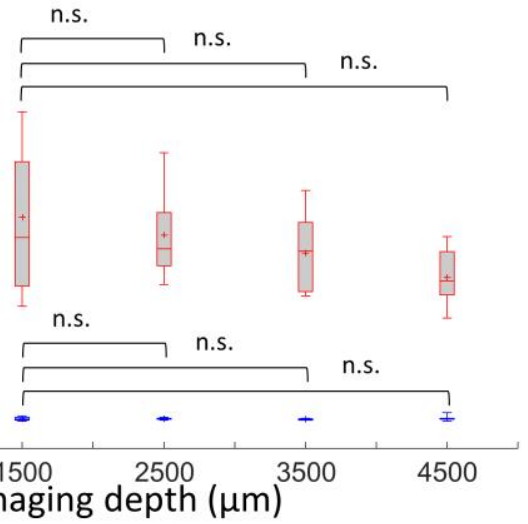
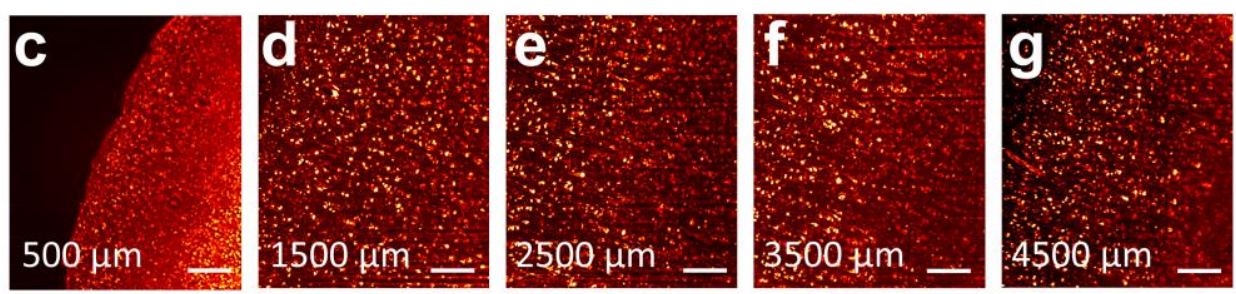

Figure 2: Cell body signal and background tissue signal over depth. (a) 3D rendering of $4.5 \mathrm{~mm}$ deep (referenced to tissue surface) LSFM imaging stack of MASH-NR stained tissue. Note that for analysis, the start of the tissue was considered as $0 \mu \mathrm{m}$ depth, not the start of the volume as indicated by the yellow line. (b) Mean, median and spread $(n=15)$ for neuronal cell body signal (red boxplots) and non-neuronal tissue background signal (blue boxplots) at several depths in a. (c-g) Images indicated at $500 \mu \mathrm{m}, 1500 \mu \mathrm{m}, 2500 \mu \mathrm{m}, 3500 \mu \mathrm{m}$ and $4500 \mu \mathrm{m}$ imaging depth. Scale bars a: $1 \mathrm{~mm}$; c-g: $200 \mu \mathrm{m}$.

Running image depth-stacks for green spectrum MASH-AO (Suppl. Video 2) and far-red spectrum MASH-MB cell body labels, also show (Suppl. Video 3-5) that signal-to-background contrast is maintained to 3-4 mm of imaging depth. Some signal decrease and slight blurring is visible deeper into the samples and further away from the light- 
sheet illumination side due to an increase in light scattering, a wellknown effect in light-sheet imaging. Note that the MASH labels could penetrate the complete sample thickness, as a lack of penetration would show as a decrease in staining intensity in the centre of the tissue, rather than at the highest depths.

\section{Validation of MASH dyes with classical stains}

MASH cell body labelling is well co-localized with standard Nissl stain cresyl violet (CV) in thin sections showing its labelling specificity and suitability for cytoarchitecture characterisation (Fig. 3). To quantify pixel-by-pixel overlap of the MASH-NR and CV labels Phi coefficient and Jaccard-index were computed for globally optimal thresholds, which gave Phi=0.832 (at thresholds $t_{1}=53$ for $C V$ and $t_{2}=21$ for NR, arbitrary units) and Jaccard $=0.811$ (at thresholds $\mathrm{t}_{1}=41$ for $\mathrm{CV}$ and $\mathrm{t}_{2}=16$ for NR, arbitrary units). All non-corresponding pixels at these thresholds were located at the edges of cells labelled by both $\mathrm{CV}$ and MASH-NR or in image structures smaller than $4 \times 4$ pixels $(3.2 \times 3.2 \mu \mathrm{m})$. Performing the same labelling specificity validation against CV for MASH-AO (Suppl. Fig. $1 \mathrm{~d}-\mathrm{f}$ ), and MASH-MB (Suppl. Fig. $1 \mathrm{~h}-\mathrm{i}$ ), as well as a validation of the MASH-MG nuclear label against DAPI (Suppl. Fig. $1 \mathrm{j}-1$ ) showed the same high co-localisation, with Phi coefficient and Jaccard index in the range 0.6-0.8 (Suppl. Fig. 2). Since the NR, MG and MB molecules can also be used as bright-field stains in thin sections at much (100x-5000x) higher concentrations, we also validated their low concentration fluorescent labelling against the 
high concentration bright-field labelling in the same thin sections (Suppl. Fig. 3), which again show very high co-localisation.

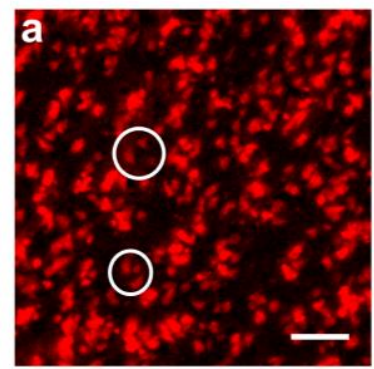

MASH-NR

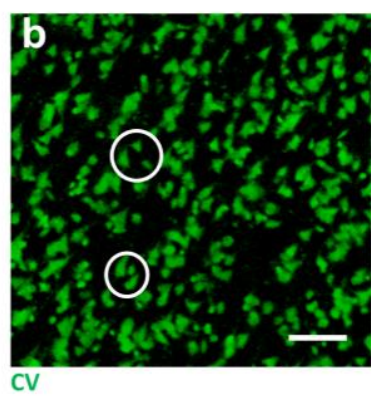

CV

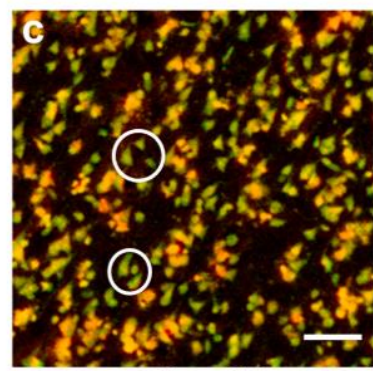

MASH-NR Phi-coefficient: 0.832 CV Jaccard-index: 0.811

Figure 3: Comparison of MASH-NR and CV on a $50 \mu \mathrm{m}$ thin section imaged with epifluorescence and bright-field microscopy respectively. (a) ROI from the section stained with MASH-NR (pseudocoloured in red) and (b) with CV (pseudocoloured in green). (c) Overlay of $a$ and $b$ with corresponding Phicoefficient and Jaccard-index; circles: corresponding locations. Scale bars: $100 \mu \mathrm{m}$.

\section{Multiscale LSFM imaging on large samples}

We demonstrated the efficiency of MASH in high-throughput 3D LSFM characterisation of human cortical cytoarchitecture by applying it to large $(\sim 40 \times 30 \times 5 \mathrm{~mm})$ archival samples surrounding the calcarine sulcus (Fig. 4). The samples were large enough to be easily localized in a magnetic resonance imaging (MRI) reconstruction of the entire host occipital lobe and to contain parts of both primary (V1) and secondary (V2) visual cortical areas (Fig. 4 a). Clearing was highly effective, rendering the entire $5 \mathrm{~mm}$ thick samples transparent with a 
slight amber-like tint (Fig. 4 b, g). Labelling could be achieved over the entire depth of the samples with the far-red MASH-MB cell body stain for the anterior sample (Fig. 4 b-f) and a two-colour labelling with the red MASH-NR cell body stain and the far-red MASH-MG nuclear stain for the posterior sample (Fig. $4 \mathrm{~g}-\mathrm{i}$ ).

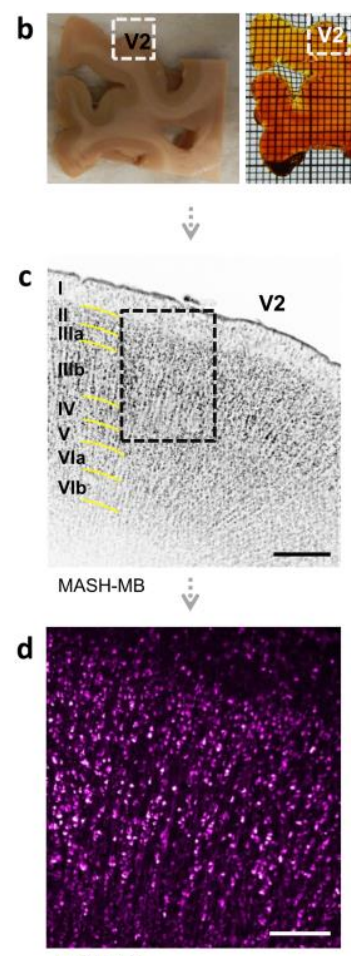

MASH-MB

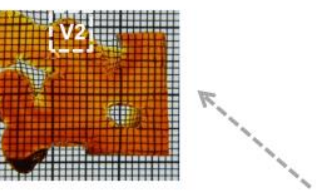

a

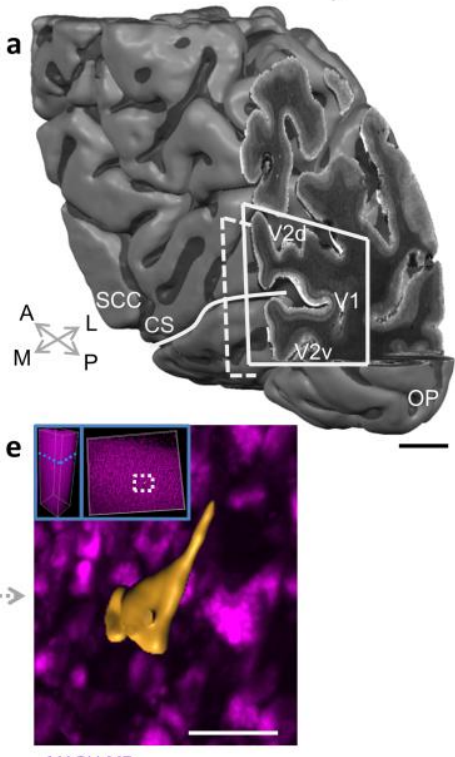

MASH-MB
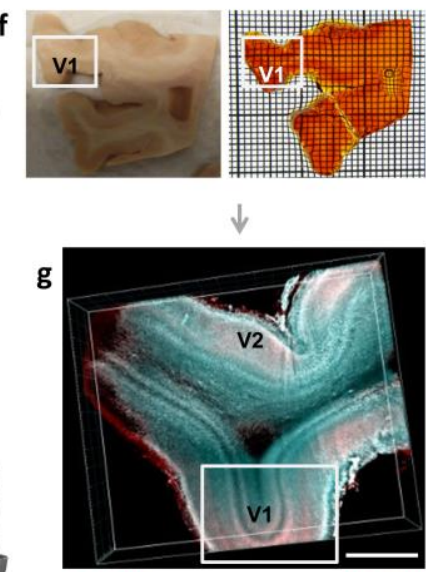

MASH-NR

MASH-MG

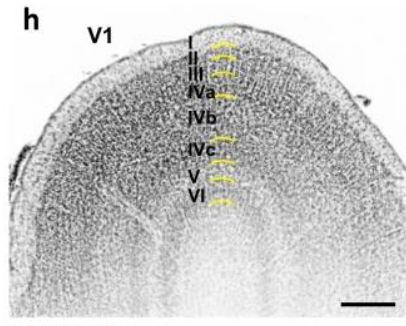

MASH-MG

Figure 4: MASH allows characterisation of human cortical cytoarchitecture in large formalin fixed samples over a large range of scales. (a) 3D MRI reconstruction of the human occipital lobe sample with primary and secondary visual cortex around the calcarine sulcus. Legend continued on the next page. 
Figure 4: Continued. V1: primary visual cortex; V2: secondary visual cortex; V2v: ventral V2; V2d: dorsal V2; CS: Calcarine Sulcus (white line); SCC: Splenium of the Corpus Callosum; OP: Occipital Pole; A: anterior; P: posterior; M: medial; L: lateral. (b) The anterior $5 \mathrm{~mm}$ thick sample (dashed line in a), before (left) and after staining and clearing (right). (c) LSFM imaging of the MASH-MB stain in V2 (inverted greyscale map) in the dashed box in $b$, with cytoarchitectonic layering characterisation (left). (d) Higher magnification LSFM of the dashed box in c. (e) a 3D surface reconstruction of a pyramidal neuron cell body from LSFM data. Inset left: depth position of the cell in the image stack, imaging depth $1066 \mu \mathrm{m}$. Inset right: horizontal position in the image stack at the white dashed box. (f) The posterior $5 \mathrm{~mm}$ thick sample (solid line in a), before (left) and after staining and clearing (right). (g) LSFM imaging of dual MASH-NR soma staining and MASHMG nucleus staining in V1 and V2 in the solid box in $\mathrm{f}$. (h) higher magnification LSFM imaging of the MASH-MG channel in the solid box in $\mathrm{g}$ (inverted orientation), with cytoarchitectonic characterisation of V1 cortical layering (middle). Thin grid (b,f): $1 \mathrm{~mm}$. Scale bars: a: $10 \mathrm{~mm}$; g: $2 \mathrm{~mm}$; c,h: $500 \mu \mathrm{m}$; d: $200 \mu \mathrm{m}$; e: $50 \mu \mathrm{m}$.

Multi-spectral LSFM imaging of layered cytoarchitecture could be performed at low magnification over 10-12 mm long stretches of V1 and V2 cortical sheet (Fig. 4 h). Higher magnification LSFM produced mesoscale imaging volumes with both the resolution to resolve single neurons and the field-of-view to contain all layers of the cortical sheet (Fig 2 c, i; Suppl. Video 4). Planes from these volumes allowed for classification of cortical layering and sub-layering, corresponding to a histological reference atlas ${ }^{17}$. They displayed distinctive cytoarchitectonic features such as the cell-poor layers IVb and V in V1 
(Fig. 4 i) and the large pyramidal cells in layer IIIb of V2 (Fig. 4 c). High magnification light-sheet imaging (Fig. 4 d; Supplementary Video 2, 3) showed soma morphology features of individual neurons in the context of a deep 3D imaging stack and allowed a coarse surface reconstruction of a pyramidal neuron cell body (Figure 4e). 


\section{Discussion}

The MASH dye protocols allow labelling of thick adult human brain samples and deep volume imaging of cytoarchitecture. The entire MASH protocol for clearing and labelling of $5 \mathrm{~mm}$ thick samples takes approximately 10 days. Moreover, MASH dye solution costs are low, less than 1 per sample. This provides the crucial scalability for the investigation of large human cortical systems and allows application in a wide range of lab environments. MASH is capable of clearing and labelling adult human archival brain samples, even after prolonged storage in formalin (current samples had been fixed for 14 to 30 months), making it applicable to tissue stored in brain banks rather than being limited to fresh or freshly frozen tissue. Additionally, they could be combined with deeply penetrating small-molecule pathology labels to investigate human diseases in their full cytoarchitectonic context as recently demonstrated e.g. by Liebmann et al. ${ }^{11}$. Earlier studies using whole-mount cytoplasmic labelling with antibodies or cresyl violet on cleared human brain samples ${ }^{18,19}$ either report over a year of processing time for $\sim 5 \times 5 \times 5 \mathrm{~mm}$ of tissue or report reliable labelling to much lower depth $(<=1 \mathrm{~mm})$.

MASH was applied here to human occipital (e.g., Fig. 4) and temporal (Suppl. Video 5) neocortical tissue. Human primary visual area is among the most myelinated cortex in the human brain. In addition, even white matter regions showed good transparency after clearing (see Fig. 4 b, g; Suppl. Fig. g, h). Therefore, it is likely that application 
of MASH to other brain areas, such as strongly myelinated cerebellum, thalamus or brainstem, is likely to yield results of similar quality.

The two low corrosive MASH RIMS can be adapted to various RIs which potentially allows their application in a wider range of optical clearing protocols. Furthermore, samples can be stored long-term in MASH RIMS before imaging because they maintain transparency and fluorescence and do not solidify at low temperatures $\left(2^{\circ} \mathrm{C}\right.$ to $\left.7^{\circ} \mathrm{C}\right)$.

Immunohistochemistry has been combined successfully with iDISCO ${ }^{11,13}$ upon which the clearing approach in MASH is based, showing that it is in principle feasible to combine MASH with antibody labelling. In addition, $\mathrm{AO}$ and $\mathrm{NR}$ have been used in the past as counterstains on standard histological sections for fluorescent tracers ${ }^{9}$, indicating that they could also act as counterstains in thick cleared specimen. The usefulness of AO and NR as counterstains for immunohistochemistry or fluorescent proteins might be limited due to the relatively low $\mathrm{pH}$ used here. It should be noted, however, that first, even though the staining gave the best contrast around a $\mathrm{pH}$ of 4 , it is possible to use especially $\mathrm{AO}$ at $\mathrm{pH} 7.4$ as well, which is a more commonly used $\mathrm{pH}$ for antibody labelling. Second and more importantly, MG has been demonstrated to work at $\mathrm{pH} 7.4^{8}$ and $\mathrm{MB}$ has been used at $\mathrm{pH} 7.4$ throughout this work. This renders the application of $\mathrm{MG}$ and $\mathrm{MB}$ as a counterstaining compatible with immunohistochemistry or transgenic labels likely. The full 
combination of specific antibodies and MASH labels needs to be investigated in depth in future work.

While antibody labelling would provide highly specific information, their combination with the MASH protocol would likely limit the achievable sample dimensions. Antibodies have limited penetration in thick specimen, mainly caused by the large size of these molecules. Furthermore, their penetration depth seems to be highly variable, depending on the antigen and the antibody. For instance, in a CLARITY based study ${ }^{19}$ penetration over 1-2 weeks varied from $1.2 \mathrm{~mm}$ to $5 \mathrm{~mm}$ depth, depending on the particular antibody for approximately cubeshaped samples of human brain tissue. In contrast, MASH small molecule dyes can penetrate several millimetres into large samples within days.

A crucial frontier in modern neuroscience is the creation of 3dimensional cytoarchitectonic reference maps and atlases to provide anatomical context for the interpretation neuroimaging results at cellular resolution ${ }^{20-22}$. We demonstrated LSFM volume microscopy imaging over a variety of scales with a commercially available lightsheet system, both at high resolution and at large field-of-view. At the resolution limit $(\sim 0.5 \times 0.5 \mu \mathrm{m}$ lateral, $4 \mu \mathrm{m}$ axial $)$ of the employed commercial light-sheet microscope the imaging approached cellular resolution, as exemplified by the surface reconstruction of a large pyramidal cell body. However, for reliable single cell reconstructions in the future an improvement in the axial resolution would be needed. 
Moreover, light-sheet microscope architectures with horizontal sideillumination of the sample can only image the extent of the sample into which the light-sheet illumination will penetrate before scattering (several millimetres). The effect of this can be observed e.g. in Supplementary Video 3-5 in the tissue parts furthest away from the illumination direction of the light-sheet. In principle, some effects of scattering can be corrected in post-processing by e.g. flat-field correction or deconvolution. However, in the future, these problems could be fundamentally alleviated by advances of LSFM technology, which would improve both resolution and imaging extent in large samples. Promising in this regard are LSFM architectures such as the dual inverted selective plane illumination microscopy (diSPIM) system geometry $^{23}$ or theta light-sheet system ${ }^{24}$, which illuminate and image the sample from above, allowing imaging in thick and arbitrarily wide samples with an automated stage. Moreover, they can achieve high near isotropic resolution, either through multi-view deconvolution or by intersecting two light-sheets in a line illumination profile. In order to open new possibilities of cellular level volume imaging of entire human cortical subsystems, such light-sheet imaging technology would have to be combined with advances in highly scalable data processing including large-volume image stitching ${ }^{25}$ and automated cell counting ${ }^{26,27}$. If successful, combining the resulting cell-level maps with other imaging modalities, such as MRI (c.f. Figure 4), could provide correlative multi-modal data on the architecture in the human cortex. 


\section{References}

1 Wagstyl, K. et al. Mapping Cortical Laminar Structure in the 3D BigBrain. Cerebral cortex 28, 2551-2562, doi:10.1093/cercor/bhy074 (2018).

2 Chung, K. et al. Structural and molecular interrogation of intact biological systems. Nature 497, 332-337, doi:10.1038/nature12107 (2013).

3 Dodt, H. U. et al. Ultramicroscopy: three-dimensional visualisation of neuronal networks in the whole mouse brain. Nature methods 4, 331-336, doi:10.1038/nmeth1036 (2007).

$4 \quad$ Erturk, A. et al. Three-dimensional imaging of solvent-cleared organs using 3DISCO. Nature protocols 7, 1983-1995, doi:10.1038/nprot.2012.119 (2012).

$5 \quad$ Kubota, S. I. et al. Whole-Body Profiling of Cancer Metastasis with SingleCell Resolution. Cell reports 20, 236-250, doi:10.1016/j.celrep.2017.06.010 (2017).

6 Renier, N. et al. iDISCO: a simple, rapid method to immunolabel large tissue samples for volume imaging. Cell 159, 896-910, doi:10.1016/j.cell.2014.10.010 (2014).

7 Susaki, E. A. et al. Whole-brain imaging with single-cell resolution using chemical cocktails and computational analysis. Cell 157, 726-739, doi:10.1016/j.cell.2014.03.042 (2014).

8 Prieto, D., Aparicio, G., Morande, P. E. \& Zolessi, F. R. A fast, low cost, and highly efficient fluorescent DNA labeling method using methyl green. Histochemistry and cell biology 142, 335-345, doi:10.1007/s00418-0141215-0 (2014).

9 Schmued, L. C., Swanson, L. W. \& Sawchenko, P. E. Some fluorescent counterstains for neuroanatomical studies. J Histochem Cytochem 30, 123-128, doi:10.1177/30.2.6174560 (1982).

10 Pan, C. et al. Shrinkage-mediated imaging of entire organs and organisms using uDISCO. Nature methods 13, 859-867, doi:10.1038/nmeth.3964 (2016). 
11 Liebmann, T. et al. Three-Dimensional Study of Alzheimer's Disease Hallmarks Using the iDISCO Clearing Method. Cell reports 16, 1138-1152, doi:10.1016/j.celrep.2016.06.060 (2016).

12 Costantini, I. et al. A versatile clearing agent for multi-modal brain imaging. Scientific reports 5, 9808, doi:10.1038/srep09808 (2015).

13 Renier, N. et al. Mapping of Brain Activity by Automated Volume Analysis of Immediate Early Genes. Cell 165, 1789-1802, doi:10.1016/j.cell.2016.05.007 (2016).

14 Klingberg, A. et al. Fully Automated Evaluation of Total Glomerular Number and Capillary Tuft Size in Nephritic Kidneys Using Lightsheet Microscopy. Journal of the American Society of Nephrology : JASN 28, 452459, doi:10.1681/ASN.2016020232 (2017).

15 Sengupta, S. et al. High resolution anatomical and quantitative MRI of the entire human occipital lobe ex vivo at 9.4T. Neurolmage 168, 162-171, doi:https://doi.org/10.1016/j.neuroimage.2017.03.039 (2018).

16 Schindelin, J. et al. Fiji: an open-source platform for biological-image analysis. Nature methods 9, 676-682, doi:10.1038/nmeth.2019 (2012).

17 von Economo, C. \& Koskinas, G. Die Cytoarchitektonik der Hirnrinde des Erwachsenen Menschen: Textband und Atlas mit 112 Mikrophotographischen Tafeln. (Springer, 1925).

18 Lai, H. M. et al. Next generation histology methods for three-dimensional imaging of fresh and archival human brain tissues. Nature communications 9, 1066, doi:10.1038/s41467-018-03359-w (2018).

19 Morawski, M. et al. Developing 3D microscopy with CLARITY on human brain tissue: Towards a tool for informing and validating MRI-based histology. Neuroimage 182, 417-428, doi:10.1016/j.neuroimage.2017.11.060 (2018).

20 Amunts, K. et al. Interoperable atlases of the human brain. Neurolmage 99, 525-532, doi:10.1016/j.neuroimage.2014.06.010 (2014).

21 Amunts, K. et al. BigBrain: an ultrahigh-resolution 3D human brain model. Science 340, 1472-1475, doi:10.1126/science.1235381 (2013). 
22 Glasser, M. F. et al. A multi-modal parcellation of human cerebral cortex. Nature 536, 171-178, doi:10.1038/nature18933 (2016).

23 Kumar, A. et al. Dual-view plane illumination microscopy for rapid and spatially isotropic imaging. Nature protocols 9, 2555-2573, doi:10.1038/nprot.2014.172 (2014).

24 Migliori, B. et al. Light-sheet theta microscopy for rapid high-resolution imaging of large biological samples. BMC biology 16, 57, doi:10.1186/s12915-018-0521-8 (2018).

25 Preibisch, S., Saalfeld, S., Schindelin, J. \& Tomancak, P. Software for beadbased registration of selective plane illumination microscopy data. Nature methods 7, 418-419, doi:10.1038/nmeth0610-418 (2010).

26 Silvestri, L. et al. Quantitative neuroanatomy of all Purkinje cells with lightsheet microscopy and high-throughput image analysis. Frontiers in neuroanatomy 9, 68, doi:10.3389/fnana.2015.00068 (2015).

27 Murakami, T. C. et al. A three-dimensional single-cell-resolution wholebrain atlas using CUBIC-X expansion microscopy and tissue clearing. Nature neuroscience 21, 625-637, doi:10.1038/s41593-018-0109-1 (2018). 


\section{Appendix Chapter 2}

Supplementary Video 1. 3D rendering of $500 \mu \mathrm{m}$ deep LSFM image stack of MASH-AO stain in TDE/CA of the superficial cortical layers

Supplementary Video 2. $3.4 \mathrm{~mm}$ deep LSFM image stack of MASH-AO stain in TDE/CA of the superficial cortical layers. Bottom left: imaging depth in $\mu \mathrm{m}$. Scale bar (bottom right): $200 \mu \mathrm{m}$.

Supplementary Video 3. $3.7 \mathrm{~mm}$ deep LSFM image stack of MASH-MB stain in TDE/CA of layer I (top right), layer II/III (middle) and layer IV (bottom left) in V2 in inverted grayscale colourmap. Bottom left: imaging depth in $\mu \mathrm{m}$. Scale bar (bottom right): $200 \mu \mathrm{m}$.

Supplementary Video 4. $3.9 \mathrm{~mm}$ deep LSFM image stack of MASH-MB stain in TDE/CA of the entire cortical depth in V2 in inverted grayscale colourmap. Bottom left: imaging depth in $\mu \mathrm{m}$. Scale bar (bottom right): $300 \mu \mathrm{m}$.

Supplementary Video 5. $3.0 \mathrm{~mm}$ deep LSFM image stack of MASH-MB stain in TDE/CA of the entire cortical depth in temporal cortex in inverted grayscale colourmap. Bottom left: imaging depth in $\mu \mathrm{m}$. Scale bar (bottom right): $500 \mu \mathrm{m}$. 

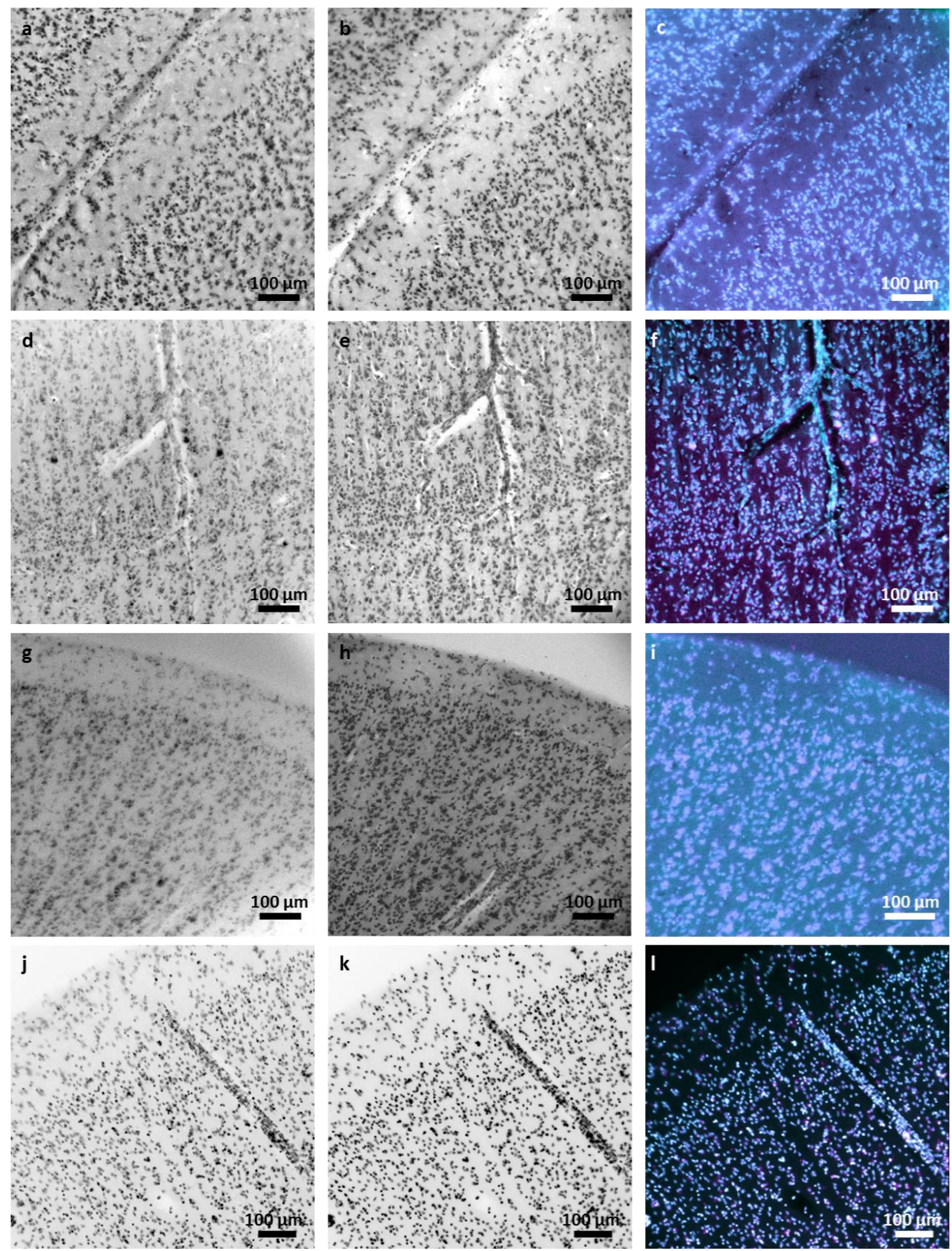
Supplementary Figure 1: Validation of MASH cytoarchitectonic dyes with established labels (prior page). Fluorescent images were inverted for better comparison with bright-field images on conventional Nissl staining. (a) MASHNR stain, (d) MASH-AO stain and (g) MASH-MB stain. (b, e and h) standard bright-field Nissl stain with cresyl violet on the same sections as shown in a, d, and $\mathbf{g}$, respectively. (j) Section stained for nuclei with MASH-MG and counterstained with DAPI (k). (c, $\mathbf{f}, \mathbf{i}$ and $\mathbf{~})$. Overlay of the MASH labels in the first column (a, d, $\mathbf{g}$ and $\mathbf{j}$ ) in magenta and the control stains in the second column ( $\mathbf{b}, \mathbf{e}, \mathbf{h}$ and $\mathbf{k}$ ) in cyan. Note the high congruency of the labelled structures between the MASH dyes and their respective control staining. 


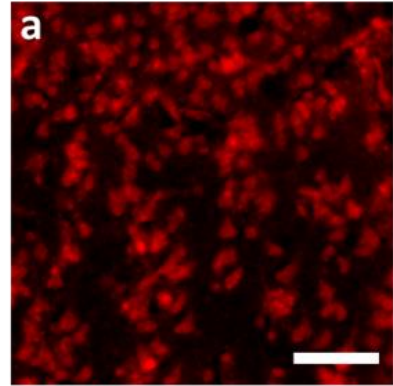

MASH-AO

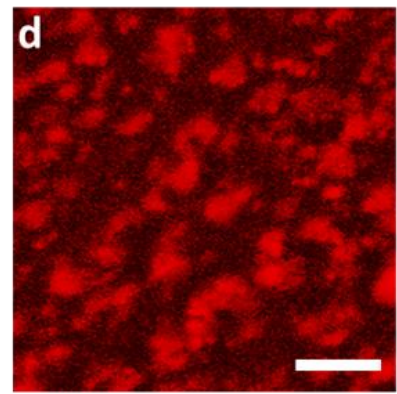

MASH-MB

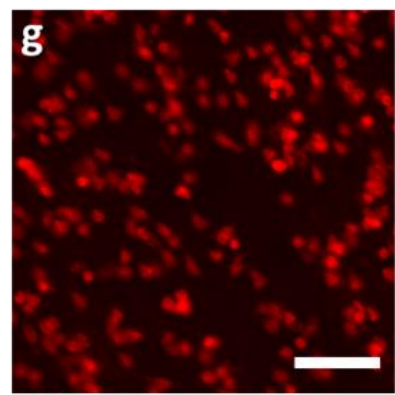

MASH-MG

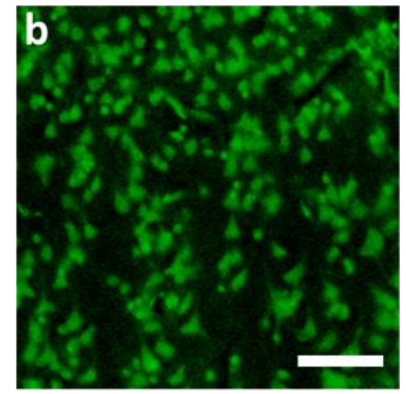

CV

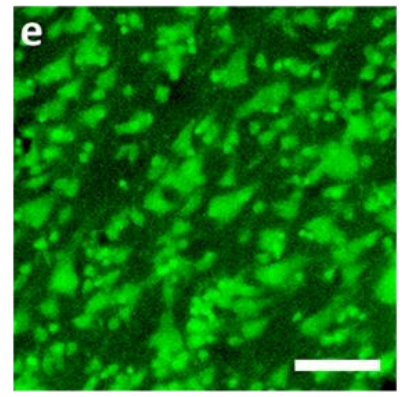

CV

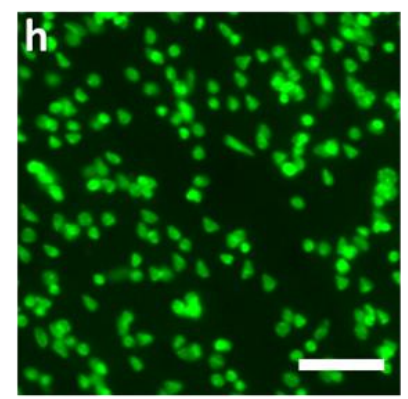

DAPI
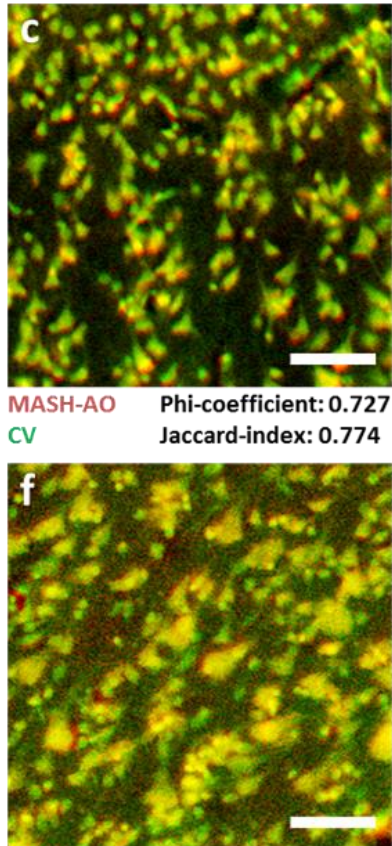

MASH-MB Phi-coefficient: $\mathbf{0 . 7 3 0}$ CV Jaccard-index: 0.734

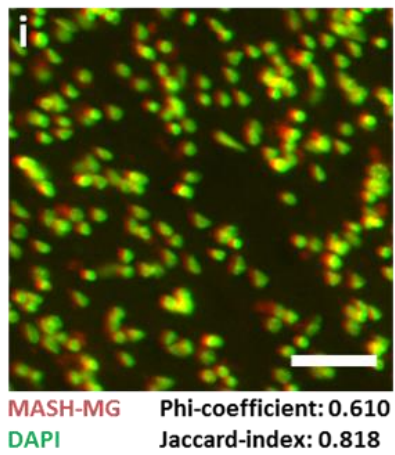

Supplementary Figure 2: Quantitative assessment of co-localization between MASH stainings and conventional staining protocols for Nissl and nuclei. All panels show magnified regions from the images in supplementary figure 1. MASH$\mathrm{AO}$ stain (a) and cresyl violet nissl stain (CV, b) show a high degree of co-localization as seen in the overlay (c) and in the pixel-by-pixel overlap as assessed with the Jaccard-index and the Phi coefficient. Legend continued on the next page. 
Supplementary Figure 2: Continued. The same comparison for MASH-MB and CV is shown in ( $\mathbf{d}-\mathbf{f})$ and for the nuclear labels MASH-MG and DAPI in ( $\mathbf{g}-\mathbf{i})$. For every panel MASH stainings have been pseudocoloured red and the respective control green. In chase of CV, which was imaged in bright field mode, the pixel values have been inverted. Scale bar: $50 \mu \mathrm{m}$ in all panels.
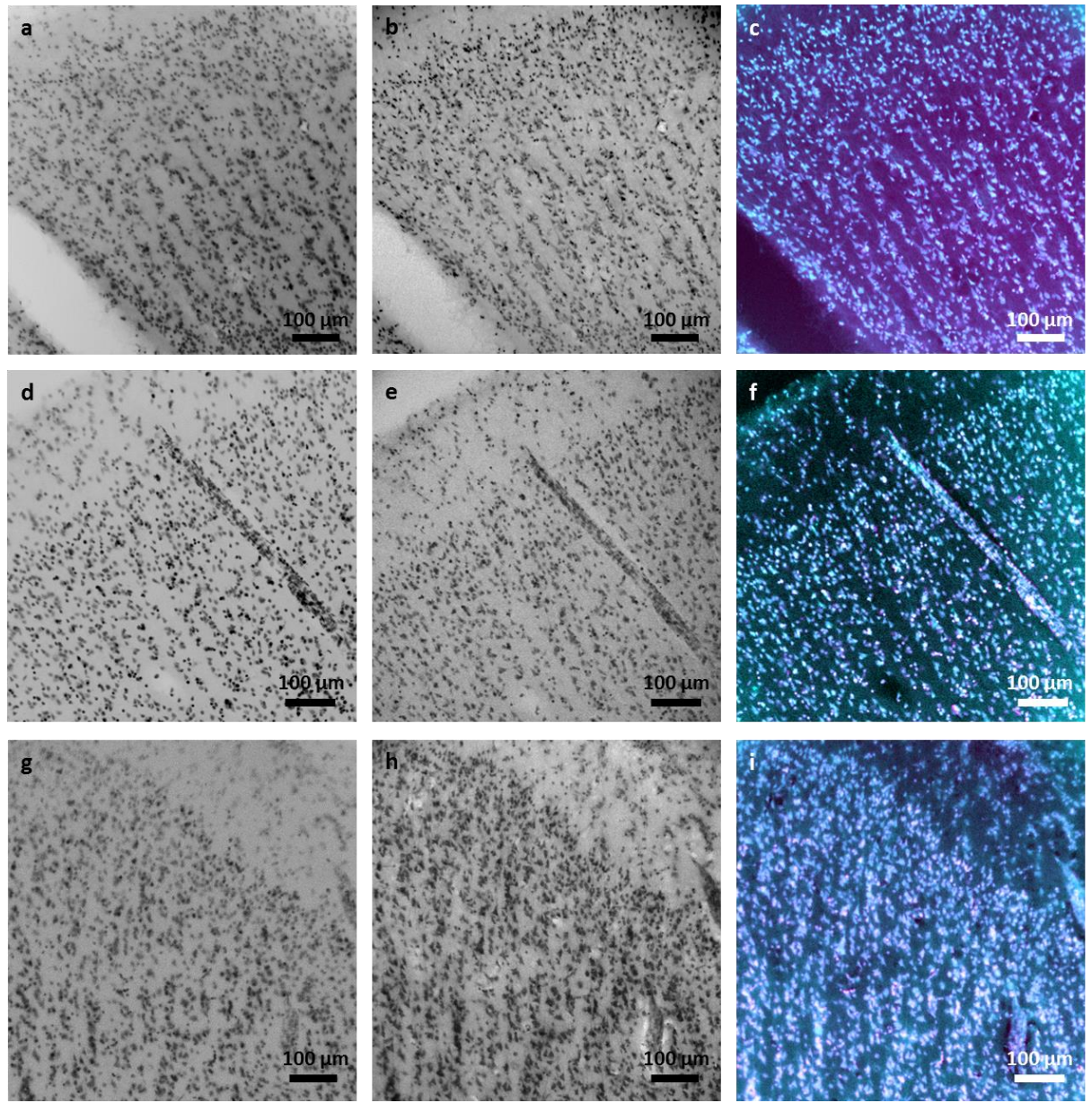
Supplementary Figure 3: Validation of MASH stains with traditional brightfield microscopy using the same dye in much (100x-5000x) higher concentration (prior page). Fluorescent MASH label images were inverted for better comparison with bright-field images. (a) MASH-NR stain (b) Bright-field stain with 100x higher concentration of neutral red on the same section. (c) The overlay of MASH-MG in magenta and the bright-field image in cyan shows that the low concentration of the dye used for MASH (magenta) is sufficient to label all structures that are visible in the traditional bright-field stain (cyan). (d) MASH-MG stain (e) Bright-field stain with 5000x higher concentration of methyl green on the same section. (f) The overlay of MASH-MG in magenta and the bright-field image in cyan shows labelling of the same structures. $(\mathrm{g})$ MASH-MB stain (h) Bright-field stain with 1000x higher concentration of methylene blue on the same section. (i) The overlay of MASH-MB in magenta and the bright-field image in cyan again shows a high congruency of labelled structures. 


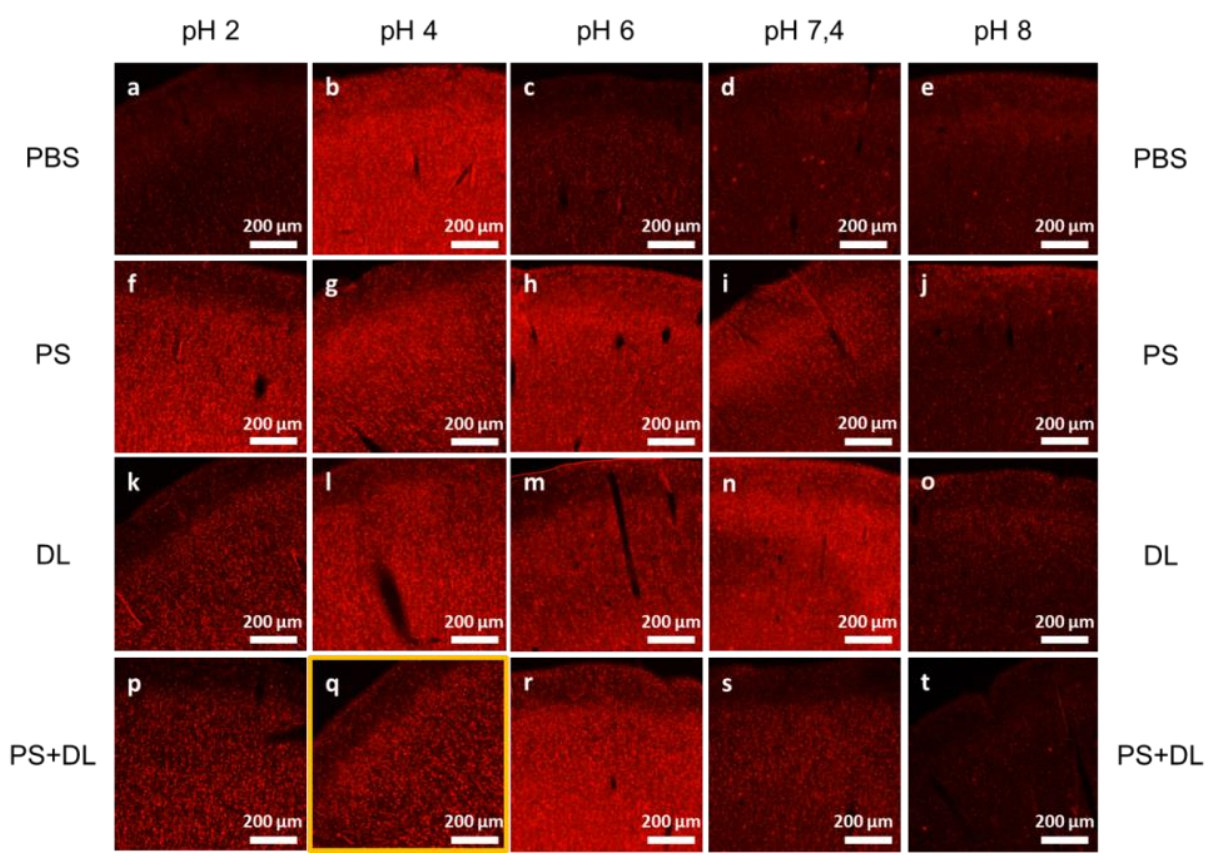

Supplementary Figure 4: Screening for optimal staining conditions for MASHNR. $50 \mu \mathrm{m}$ sections of human brain tissue were stained with $0.001 \%$ neutral red and pretreated with several procedures prior to staining. $(a-e)$ Control sections incubated in PBS at respective $\mathrm{pH}$ for $30 \mathrm{~min}$. ( $\mathbf{f}-\mathbf{j}$ ) Sections were incubated for $15 \mathrm{~min}$ in $50 \%$ filtered, aqueous potassium pyrosulfite solution before staining. ( $(\mathbf{k}-\mathbf{0})$ Sections were delipidated with $70 \%, 100 \%$ and $70 \%$ methanol in $\mathrm{H} 2 \mathrm{O}$ for 5 min each. (p - t) Samples were first delipidated with $70 \%, 100 \%$ and $70 \%$ methanol in $\mathrm{H} 2 \mathrm{O}$ for 5 min each, followed by 15 min incubation in $50 \%$ filtered, aqueous potassium pyrosulfite solution prior to staining. Legend is continued on the next page. 
Supplementary Figure 4: Continued. After pretreatment, samples were washed for 5 min in $\mathrm{H} 2 \mathrm{O}$. All samples were stained in PBS buffered staining solution respectively for $1 \mathrm{~h}$ each. $\mathrm{pH}$ of the buffered solution was as follows: (a, f, k, and p) pH 2; (b, g, l, and q) pH 4; (c, h, m, and r) pH 6; (d, i, n, and s) pH 7.4; $(\mathrm{e}, \mathrm{j}, \mathrm{o}$, and $\mathrm{t}) \mathrm{pH}$ 8. Best results were obtained with the combined delipidation and preatreatment of the tissue with potassium pyrosulfite at $\mathrm{pH}$ 4 (q, in orange box).

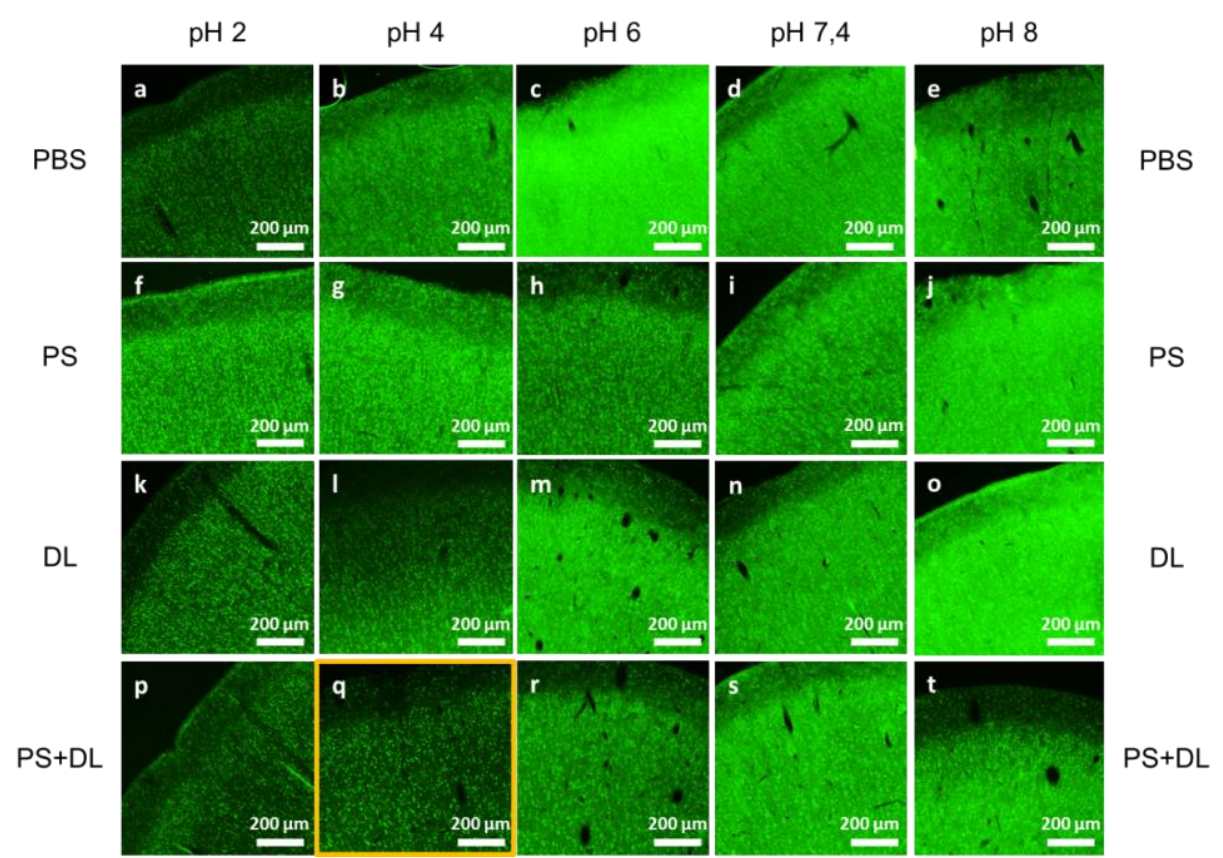

Supplementary Figure 5: Screening for optimal staining conditions for MASHAO. $50 \mu \mathrm{m}$ sections of human brain tissue were stained with $0.001 \%$ acridine orange and pretreated with several procedures prior to staining. (a - e) Control sections incubated in PBS at respective $\mathrm{pH}$ for $30 \mathrm{~min}$. (f $-\mathbf{j}$ ) Sections were incubated for $15 \mathrm{~min}$ in $50 \%$ filtered, aqueous potassium pyrosulfite solution before staining. Legend is continued on the next page. 
Supplementary Figure 5: Continued. $(\mathbf{k}-\mathbf{0})$ Sections were delipidated with $70 \%, 100 \%$ and $70 \%$ methanol in $\mathrm{H} 2 \mathrm{O}$ for 5 min each. ( $\mathbf{p}-\mathbf{t})$ Samples were first delipidated with $70 \%, 100 \%$ and $70 \%$ methanol in $\mathrm{H} 2 \mathrm{O}$ for 5 min each, followed by 15 min incubation in $50 \%$ filtered, aqueous potassium pyrosulfite solution prior to staining. After pretreatment, samples were washed for $5 \mathrm{~min}$ in $\mathrm{H} 2 \mathrm{O}$. All samples were stained in PBS buffered staining solution respectively for $1 \mathrm{~h}$ each. $\mathrm{pH}$ of the buffered solution was as follows: (a, $\mathbf{f}, \mathbf{k}$, and p) pH 2; (b, g, I, and q) pH 4; (c, h, m, and r) pH 6; (d, i, n, and s) pH 7.4; (e, j, o, and t) $\mathrm{pH}$ 8. Staining with $\mathrm{pH} 2$ and 4 resulted in strong labelling independent of pretreatment. Best conditions with very little background were obtained with combined delipidation and potassium pyrosulfite pretreatment at $\mathrm{pH} 4$ (q, in orange box). 

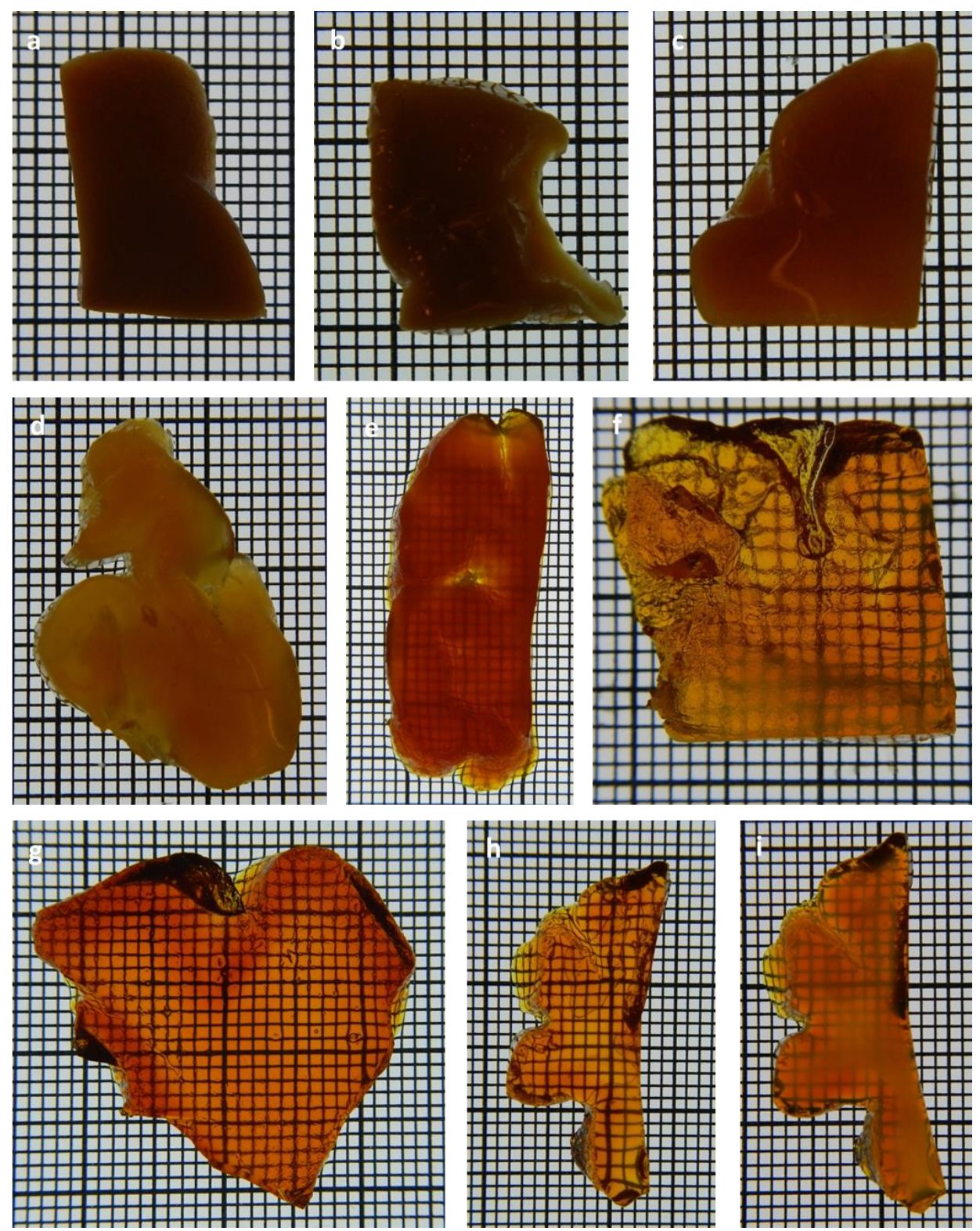
Supplementary Figure 6: Effect of different refractive indices (Rl's) on transparency of adult human archival brain tissue cleared with iDISCO+ (prior page). (a) Sample incubated in PBS after clearing $(R I=1.33)$. (b) $R I=1.44$ with an aqueous solution (80\% glycerol in PBS) (c) RI $=1.47$ with a non-aqueous solution (mineral oil). (d) Immersion in 100\% 2,2'-Thiodiethanol (TDE; RI = 1.52). (e) Incubation in pure wintergreen oil (WGO; $R I=1.54)$. ( $f-h)$ Brain tissue incubated in different RI matching media with an RI of 1.56, all of which provide good transparency with a slight amber colour. (f) Ethyl cinnamate (ECi). (g) Incubated in a mixture of $72 \% \mathrm{WGO}$ and $28 \%$ trans-cinnamaldehyde (WGO/CA) (h) 62\% TDE and 38\% CA (TDE/CA). (i) Incubation in 38\% TDE/ $62 \%$ CA solution with a RI of 1.58. Even slight mismatches in RI (e.g. 0.02 lower, in e, or higher, in i) are seen to lead to drastic reduction in transparency. Thin grid: $1 \mathrm{~mm}$ in all figures. 
Supplementary Table 1. Samples, tissue processing and imaging parameters corresponding to figures and videos.

\begin{tabular}{|c|c|c|c|c|c|c|c|c|c|c|c|c|}
\hline Fig. & $\begin{array}{l}\text { Sample } \\
\text { Number }\end{array}$ & Subject & $\begin{array}{l}\text { Fixation time } \\
\text { [months] }\end{array}$ & $\begin{array}{l}\text { sample } \\
\text { thickness [mm] }\end{array}$ & Dye & $\begin{array}{l}\text { Incubation } \\
\text { time [days] }\end{array}$ & $\mathrm{pH}$ & Medium & Modality & Ex. & Em. & Magnification \\
\hline $\begin{array}{r}\text { 1e, } 4 b-e, \\
\text { Suppl. } \\
\text { Video } 3,4\end{array}$ & 89 & Subject 1 & 14 & 5 & $\mathrm{MB}$ & 5 & 7.4 & TDE/CA & LSFM & 630 & 680 & $12.6 x, 5 x$ \\
\hline $1 \mathrm{f}, 4 \mathrm{f}-\mathrm{h}$ & 91 & Subject 1 & 14 & 5 & $\mathrm{NR}, \mathrm{MG}$ & 5 & 4 & TDE/CA & LSFM & $\begin{array}{r}560 \\
630\end{array}$ & $\begin{array}{r}620 \\
680\end{array}$ & $2 x, 4 x$ \\
\hline $2 a-g$ & 101 & Subject 2 & 30 & $\mathrm{~N}$ & NR, DAPI & 5 & 4 & TDE/CA & LSFM & 560 & 620 & $12.6 x$ \\
\hline $1 a$ & 107 & Subject 2 & 30 & 2 & $\begin{array}{r}\text { AO, } \\
\text { DAPI }\end{array}$ & 2 & 4 & WGO/CA & TPM & 800 & 550 & $20 x$ \\
\hline $1 b$ & 108 & Subject 2 & 30 & 2 & $\begin{array}{r}\text { AO, } \\
\text { DAPI }\end{array}$ & 2 & 4 & WGO/CA & TPM & 800 & 550 & $20 x$ \\
\hline $1 d$ & 109 & Subject 2 & 30 & 2 & $\begin{array}{r}\text { AO, } \\
\text { DAPI }\end{array}$ & 2 & 4 & TDE/CA & TPM & 800 & 550 & $20 x$ \\
\hline $1 c$ & 110 & Subject 2 & 30 & $\mathrm{~N}$ & NR, DAPI & 2 & 4 & TDE/CA & TPM & 800 & 600 & $20 x$ \\
\hline
\end{tabular}




\begin{tabular}{|c|c|c|c|c|c|c|c|c|c|c|c|}
\hline $\begin{array}{r}3 a-c, \\
\text { Suppl. 1a- } \\
c\end{array}$ & 111 & $\begin{array}{r}\text { Subject } \\
2,1 \\
\text { section }\end{array}$ & 30 & 0.05 & NR, CV & n.a. & 4 & $\begin{array}{r}\text { Slide: } \\
\text { Kaiser's } \\
\text { glycerol } \\
\text { gelatine, } \\
\text { Entellan }\end{array}$ & $\begin{array}{r}\text { epifluore } \\
\text { scence, } \\
\text { bright- } \\
\text { field }\end{array}$ & 560 & 600 \\
\hline $\begin{array}{r}\text { Suppl. 1d- } \\
\text { f, Suppl. } \\
2 a-c\end{array}$ & 112 & $\begin{array}{r}\text { Subject } \\
2,1 \\
\text { section }\end{array}$ & 30 & 0.05 & $\mathrm{AO}, \mathrm{CV}$ & n.a. & 4 & $\begin{array}{r}\text { Slide: } \\
\text { Kaiser's } \\
\text { glycerol } \\
\text { gelatine, } \\
\text { Entellan }\end{array}$ & $\begin{array}{r}\text { epifluore } \\
\text { scence, } \\
\text { bright- } \\
\text { field }\end{array}$ & 488 & 550 \\
\hline $\begin{array}{r}\text { Suppl. 1g- } \\
\text { i, Suppl. } \\
2 d-f\end{array}$ & 113 & $\begin{array}{r}\text { Subject } \\
2,1 \\
\text { section }\end{array}$ & 30 & 0.05 & $\mathrm{MB}, \mathrm{CV}$ & n.a. & 7.4 & $\begin{array}{r}\text { Slide: } \\
\text { Kaiser's } \\
\text { glycerol } \\
\text { gelatine, } \\
\text { Entellan }\end{array}$ & $\begin{array}{r}\text { epifluore } \\
\text { scence, } \\
\text { bright- } \\
\text { field }\end{array}$ & 630 & 680 \\
\hline $\begin{array}{l}\text { Suppl. 1j-l, } \\
\text { Suppl. 2g-i }\end{array}$ & 114 & $\begin{array}{r}\text { Subject } \\
2,1 \\
\text { section }\end{array}$ & 30 & 0.05 & $\begin{array}{l}\text { MG, } \\
\text { DAPI }\end{array}$ & n.a. & 4 & $\begin{array}{r}\text { Slide: } \\
\text { Kaiser's } \\
\text { glycerol } \\
\text { gelatine, } \\
\text { Entellan }\end{array}$ & $\begin{array}{r}\text { epifluore } \\
\text { scence, } \\
\text { bright- } \\
\text { field }\end{array}$ & $\begin{array}{r}630 \\
405\end{array}$ & $\begin{array}{r}680 \\
460\end{array}$ \\
\hline
\end{tabular}




\begin{tabular}{|c|c|c|c|c|c|c|c|c|c|c|c|}
\hline $\begin{array}{r}\text { Suppl. 3a- } \\
\text { c }\end{array}$ & 115 & $\begin{array}{r}\text { Subject } \\
2,1 \\
\text { section }\end{array}$ & 30 & 0.05 & NR & n.a. & 4 & $\begin{array}{r}\text { Slide: } \\
\text { Kaiser's } \\
\text { glycerol } \\
\text { gelatine, } \\
\text { Entellan }\end{array}$ & $\begin{array}{r}\text { epifluore } \\
\text { scence, } \\
\text { bright- } \\
\text { field }\end{array}$ & 560 & 600 \\
\hline Suppl. 3d-f & 116 & $\begin{array}{r}\text { Subject } \\
2,1 \\
\text { section }\end{array}$ & 30 & 0.05 & MG & n.a. & 4 & $\begin{array}{r}\text { Slide: } \\
\text { Kaiser's } \\
\text { glycerol } \\
\text { gelatine, } \\
\text { Entellan }\end{array}$ & $\begin{array}{r}\text { epifluore } \\
\text { scence, } \\
\text { bright- } \\
\text { field }\end{array}$ & 630 & 680 \\
\hline Suppl. 3g-i & 117 & $\begin{array}{r}\text { Subject } \\
2,1 \\
\text { section }\end{array}$ & 30 & 0.05 & MB & n.a. & 7.4 & $\begin{array}{r}\text { Slide: } \\
\text { Kaiser's } \\
\text { glycerol } \\
\text { gelatine, } \\
\text { Entellan }\end{array}$ & $\begin{array}{r}\text { epifluore } \\
\text { scence, } \\
\text { bright- } \\
\text { field }\end{array}$ & 630 & 680 \\
\hline Suppl. 4 & 118 & $\begin{array}{r}\text { Subject } \\
2,20 \\
\text { sections }\end{array}$ & 30 & 0.05 & NR & n.a. & $\begin{array}{c}8- \\
\mathrm{Fe} \\
\mathrm{b}\end{array}$ & $\begin{array}{r}\text { Slide: } \\
\text { Kaiser's } \\
\text { glycerol } \\
\text { gelatine }\end{array}$ & $\begin{array}{r}\text { epifluore } \\
\text { scence }\end{array}$ & 560 & 600 \\
\hline
\end{tabular}




\begin{tabular}{|c|c|c|c|c|c|c|c|c|c|c|c|c|}
\hline Suppl. 5 & 119 & $\begin{array}{r}\text { Subject } \\
2,20 \\
\text { sections }\end{array}$ & 30 & 0.05 & $\mathrm{AO}$ & n.a. & $\begin{array}{c}8- \\
\mathrm{Fe} \\
\mathrm{b}\end{array}$ & $\begin{array}{r}\text { Slide: } \\
\text { Kaiser's } \\
\text { glycerol } \\
\text { gelatine }\end{array}$ & $\begin{array}{r}\text { epifluore } \\
\text { scence }\end{array}$ & 488 & 550 & $10 x$ \\
\hline $\begin{array}{r}\text { Suppl. } \\
\text { Video } 1,2\end{array}$ & 120 & Subject 2 & 30 & 5 & $\mathrm{AO}$ & 5 & 4 & TDE/CA & LSFM & 630 & 680 & $12.6 \mathrm{x}$ \\
\hline $\begin{array}{r}\text { Suppl. } \\
\text { Video } 5\end{array}$ & 121 & Subject 3 & 3 & 5 & MB & 5 & 7.4 & TDE/CA & LSFM & 630 & 680 & \\
\hline
\end{tabular}


Supplementary Table 2. Chemical properties and hazard statements of the different substances tested as RIMS. Corrosiveness refers to the ability of the compound to dissolve plastic materials. Abbreviations: $\mathrm{CA}=$ trans-

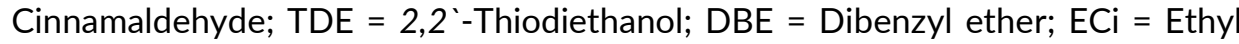
cinnamate; $\mathrm{WGO}=$ wintergreen oil/ Methyl salicylate; $\mathrm{PBS}=$ phosphate buffered saline. Corrosiveness: $0=$ no effect on any plastic tested after 7 days; $+=$ mild to moderate damage on polystyrene after 7 days, no to mild damage on polypropylene after 7 days, no effects on high-density polyethylene and tetrafluoroethylene after 7 days; ++ = moderate to heavy damage on polystyrene after several hours/days, no to mild damage on polypropylene after 7 days, no effects on high-density polyethylene and tetrafluoroethylene after 7 days; +++ = immediate damage on polystyrene, heavy to moderate damage on polypropylene after 7 days, little to no effects on high-density polyethylene and tetrafluoroethylene after 7 days.

\begin{tabular}{lrrr} 
Medium & \multicolumn{2}{c}{ MI $\left[{ }^{\circ} \mathrm{C}\right.$ ] } & Corrosiveness \\
\hline $100 \%$ CA & 1.622 & -9 to -4 & ++ \\
$38 \%$ TDE/62 \% CA & 1.58 & $-16 /$ & ++ \\
$100 \%$ DBE & 1.562 & 1.5 to 3.5 & +++ \\
$100 \%$ ECi & 1.558 & 6 to 8 & + \\
$62 \%$ TDE/38 \% CA & 1.56 & $-16 /$ & + \\
& & -9 to -4 & \\
$72 \%$ WGO/28 \% CA & 1.56 & -8 to $-7 /$ & ++ \\
$100 \%$ WGO & & -9 to -4 & ++ \\
$100 \%$ TDE & 1.536 & -8 to -7 & 0 \\
$100 \%$ MO & 1.5215 & -16 & + \\
$80 \%$ Glycerol in PBS & 1.467 & n.a. & 0 \\
PBS & 1.442 & n.a. & 0
\end{tabular}


Supplementary Table 3. Image acquisition settings for TPM and LSFM.

\begin{tabular}{|c|c|c|c|c|c|c|c|c|c|c|}
\hline Fig. & $\begin{array}{l}\text { Sample } \\
\text { Number }\end{array}$ & Dye & Modality & Excitation & Emission & Magnification & $\begin{array}{l}\text { Scan field } \\
\text { resolution [Pixels] }\end{array}$ & $\begin{array}{l}\text { Scan field } \\
\text { size }[\mu \mathrm{m}]\end{array}$ & $\begin{array}{l}\text { Pixel size } \\
{[\mu \mathrm{m}]}\end{array}$ & $\begin{array}{l}\text { Imaging } \\
\text { Depth }[\mu \mathrm{m}]\end{array}$ \\
\hline $1 \mathrm{e}$ & 89 & MB & LSFM & $630 / 30$ & $680 / 30$ & $12.6 x$ & $2560 \times 2160$ & $1318 \times 1112$ & 0.51 & 3694 \\
\hline $4 c$ & 89 & MB & LSFM & $630 / 30$ & $680 / 30$ & $5 x$ & $2560 \times 2160$ & $3328 \times 2808$ & 1.3 & 3694 \\
\hline $4 d$ & 89 & MB & LSFM & $630 / 30$ & $680 / 30$ & $12.6 \mathrm{x}$ & $2560 \times 2160$ & $1318 \times 1112$ & 0.51 & 3694 \\
\hline $4 \mathrm{e}$ & 89 & MB & LSFM & $630 / 30$ & $680 / 30$ & $12.6 \mathrm{x}$ & $2560 \times 2160$ & $1318 \times 1112$ & 0.51 & 3694 \\
\hline
\end{tabular}




\begin{tabular}{|c|c|c|c|c|c|c|c|c|c|c|}
\hline $1 \mathrm{f}$ & 91 & $\begin{array}{l}\text { NR, } \\
\text { MG }\end{array}$ & LSFM & $\begin{array}{l}560 / 40 \\
630 / 30\end{array}$ & $\begin{array}{r}620 / 60 \\
680 / 30\end{array}$ & $2 x$ & $2560 \times 2160$ & $8320 \times 7020$ & 3.25 & 3800 \\
\hline $4 g$ & 91 & $\begin{array}{l}\text { NR, } \\
\text { MG }\end{array}$ & LSFM & $\begin{array}{l}560 / 40 \\
630 / 30\end{array}$ & $\begin{array}{r}620 / 60 \\
680 / 30\end{array}$ & $4 x$ & $2560 \times 2160$ & $4160 \times 3510$ & 1.62 & 3800 \\
\hline $4 \mathrm{~h}$ & 91 & $\begin{array}{l}\text { NR, } \\
\text { MG }\end{array}$ & LSFM & $\begin{array}{l}560 / 40 \\
630 / 30\end{array}$ & $\begin{array}{r}620 / 60 \\
680 / 30\end{array}$ & $2 x$ & $2560 \times 2160$ & $8320 \times 7020$ & 3.25 & 3800 \\
\hline $2 a-e$ & 101 & $\begin{array}{r}\text { NR, } \\
\text { DAPI }\end{array}$ & LSFM & $560 / 40$ & $620 / 60$ & $12.6 x$ & $2560 \times 2160$ & $1318 \times 1112$ & 0.51 & 4700 \\
\hline $1 a$ & 107 & $\begin{array}{r}\text { AO, } \\
\text { DAPI }\end{array}$ & TPM & 800 & 550 & $20 x$ & $1024 \times 1024$ & $395 \times 395$ & 2.6 & $\begin{array}{r}\text { Single 2D } \\
\text { plane from } \\
33 \mu \mathrm{m} \\
\text { stack }\end{array}$ \\
\hline $1 \mathrm{~b}$ & 108 & $\begin{array}{r}\text { AO, } \\
\text { DAPI }\end{array}$ & TPM & 800 & 550 & $20 x$ & $1024 \times 1024$ & $395 \times 395$ & 2.6 & 33 \\
\hline
\end{tabular}




\begin{tabular}{|c|c|c|c|c|c|c|c|c|c|c|}
\hline $1 d$ & 109 & $\begin{array}{r}\text { AO, } \\
\text { DAPI }\end{array}$ & TPM & 800 & 550 & $20 x$ & $1024 \times 1024$ & $738 \times 738$ & 1.4 & $\begin{array}{r}\text { Single } 2 \mathrm{D} \\
\text { plane }\end{array}$ \\
\hline $1 \mathrm{c}$ & 110 & $\begin{array}{r}\text { NR, } \\
\text { DAPI }\end{array}$ & TPM & 800 & 600 & $20 x$ & $1024 \times 1024$ & $434 \times 434$ & 2.4 & $\begin{array}{r}\text { Single } 2 D \\
\text { plane from } \\
1860 \mu \mathrm{m} \\
\text { stack }\end{array}$ \\
\hline
\end{tabular}


Supplementary Table 4. MASH dye molecule properties. Ex.= single photon peak excitation wavelength, Em.= peak emission wavelength

\begin{tabular}{|c|c|c|c|c|c|c|c|}
\hline Dye & Labelled Structures & Molecule & PubChem CID & Molecular Formula & $\begin{array}{l}\text { Molecular } \\
\text { Weight }[\mathrm{g} / \mathrm{mol}]\end{array}$ & Ex. [nm] & Em. [nm] \\
\hline MASH-AO & $\begin{array}{r}\text { Cell bodies: Cytoplasm (RNA) } \\
\& \text { nucleus (DNA) }\end{array}$ & Acridine orange & 62344 & $\mathrm{C}_{17} \mathrm{H}_{19} \mathrm{~N}_{3}$ & 265.36 & 488 & 550 \\
\hline MASH-NR & $\begin{array}{r}\text { Cell bodies: Cytoplasm (RNA) } \\
\& \text { nucleus (DNA) }\end{array}$ & Neutral red & 11105 & $\mathrm{C}_{15} \mathrm{H}_{17} \mathrm{ClN}_{4}$ & 288.779 & 550 & 600 \\
\hline MASH-MB & $\begin{array}{r}\text { Cell bodies: Cytoplasm (RNA) } \\
\& \text { nucleus (DNA) }\end{array}$ & Methylene blue & 6099 & $\mathrm{C}_{16} \mathrm{H}_{18} \mathrm{ClN}_{3} \mathrm{~S}$ & 319.851 & 630 & 700 \\
\hline MASH-MG & Cell Nuclei (DNA) & Methyl green & 44134822 & $\begin{array}{r}\mathrm{C}_{27} \mathrm{H}_{35} \mathrm{BrClN}_{3} \cdot \\
\mathrm{ZnCl}_{2}\end{array}$ & 653.24 & 630 & 700 \\
\hline
\end{tabular}




\title{
An investigation of angioarchitecture in
}

\author{
the human visual cortex with \\ angioMASH tissue clearing and
}

\section{labelling}

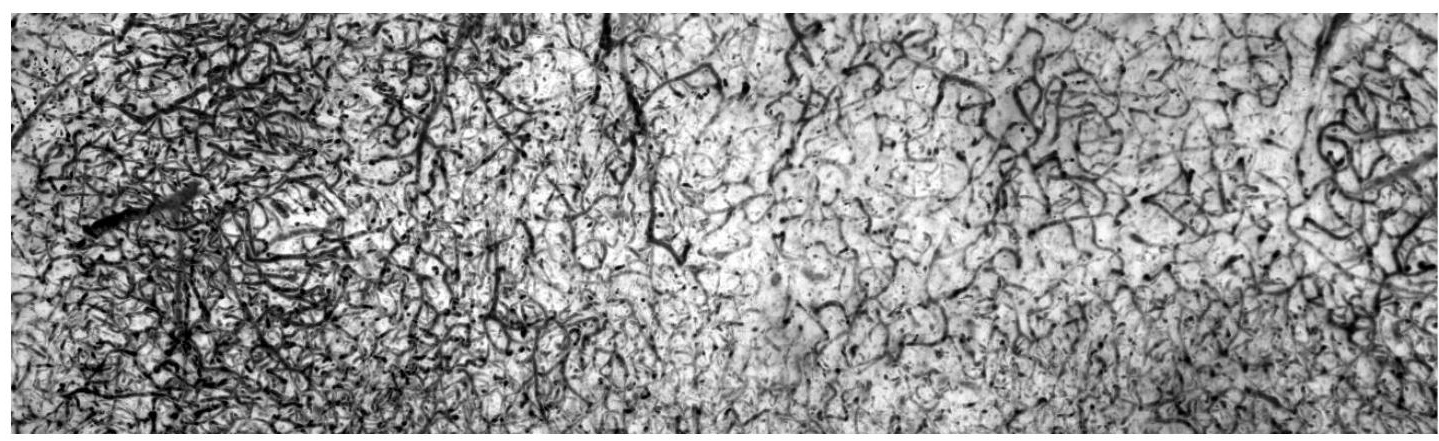

Vasculature of human occipital cortex stained with angio-MASH.

(in preparation)

Sven Hildebrand, Philipp Bethghe, Michael Capalbo, Andreas Herrler, Fritjof Helmchen, Alard Roebroeck 


\section{Abstract}

Non-invasive imaging techniques, such as ultra-high field fMRI, are intricately connected to the underlying vasculature and are approaching ever higher resolutions, towards a scale at which cortical microvascular differences will have to be taken into account. Therefore, a better understanding of the laminar distribution and interareal differences in the cortical vasculature is becoming more important. However, in comparison to cyto- and myeloarchitecture, the study of angioarchitecture has received far less attention and relatively few methods have been described to visualise the vascular network in the human brain. Here we present angioMASH, a method for double labelling angioarchitecture and cytoarchitecture in archival human brain tissue based on the cytoarchitecture labelling and optical clearing of our MASH protocol. The double labelling and optical clearing of thick human brain slices can be accomplished in a matter of $\sim 2$ weeks. We use this method to acquire multi-resolution 3D datasets of combined cyto- and angioarchitecture in large human samples covering areas V1 and V2. We demonstrate for the first time, that classical angioarchitectonic features can be visualised in 3D using tissue clearing and show differences in the vessel density and orientation over cortical depth within and between the two areas. 


\section{Introduction}

The human brain has huge metabolic demands for its functioning. In order to supply the nervous tissue with nutrients and oxygen, the brain contains an intricate vascular network of immense density and complexity. Since the commencement of Pfeifer's work over 90 years ago $^{2-4}$ and later Duvernoy ${ }^{5}$, it has become clear that the human cortex is divided into horizontal vascular layers each characterised by a distinct density and distribution of arterioles, venules and capillaries. This allows for the parcellation of the brain into different areas with different angioarchitecture, homologous to the famous brain maps based on either cyto- or myeloarchitecture, such as the Brodmann ${ }^{6}$ or von Economo and Koskinas ${ }^{1}$ maps (cytoarchitecture) and the myeloarchitectonic maps by the Vogt $\mathrm{school}^{7-13}$. However, historically the angioarchitecture of the human cortex has received far less attention as compared to its cyto- and myeloarchitecture, which are much closer to the neuronal physiology and functioning of the cortex. Yet, the vasculature contributes indirectly but crucially to the brain's function, supporting its metabolic needs and, via the blood brain barrier, protecting the nervous tissue from harmful substances. Understanding the variations of cortical vasculature in the human brain is becoming increasingly important. Not only are changes to the vasculature implicated in several neurodegenerative diseases, such as vascular dementia, the vascular network is also essential for noninvasive functional imaging modalities. Functional Near Infrared Spectroscopy (fNIRS) ${ }^{14,15}$ and functional Magnetic Resonance Imaging 
$(\mathrm{fMRI})^{16,17}$ for instance, rely on the optical and magnetic properties of blood in cortical vasculature serving as the signal source. In recent years, technological advances, particularly at ultra-high magnetic field strengths, have enabled fMRI at resolutions high enough to distinguish both laminar and columnar mesoscopic structures ${ }^{18-22}$. Since the signal of both fNIRS and fMRI is only indirectly related to neurons and at the same time intricately linked to the brain's blood flow, it is important to understand the relations between cyto- and angioarchitecture. At the same time, the degree to which different layers and areas as identified by cytoarchitecture correspond to changes in the organisation of angioarchitecture is not sufficiently understood. Although the layer-to-layer correlation of the two features within a given area seems to be $w^{2} \mathrm{k}^{23-26}$, it has been shown that the overall densities of cells and vasculature are correlated when comparing interareal averages ${ }^{25}$. Furthermore, the vasculature itself seems to be organized in a laminar rather than columnar manner, which varies visibly between cortical areas ${ }^{2-5,27}$. Individual reports differ, but generally the highest capillary density can be observed around the lower part of (cytoarchitectural) layer III to IV, with the marked exception of primary visual cortex ${ }^{5,23,24,26}$. Aside from the extensive, albeit only descriptive work of the early pioneers ${ }^{2,3,28}$, few studies have been conducted on human brain tissue so $\mathrm{far}^{23,29}$. A better understanding of the underlying anatomy could provide useful information to an imaging community aiming for ever higher field strength and resolution with fMRI techniques. 
Recent developments in histological tissue processing and microscopical imaging, now allow for fast volumetric imaging over large samples in order to address these questions in an unprecedented manner. Tissue clearing has become a widely used technique in many neuroscience labs, particularly for studies in rodents, as well as in other biomedical fields. Although initially its application to human brain tissue proved to be difficult, considerable progress has been made in recent years ${ }^{30-35}$, with for instance solventbased approaches showing especially high clearing capacity ${ }^{30,33,35}$. Therefore, rendering the tissue transparent is no longer the main challenge in the utilisation of optical clearing in human brain tissue and the focus has shifted towards more sophisticated labelling strategies $^{36,37}$ and data analysis ${ }^{38-41}$. Recently several publications have used lectins as labels for vasculature in combination with optical clearing. So far however, this has been applied mainly in the mouse brain or on relatively small and thin human brain pieces ${ }^{32,36,38,41,42}$. Crucially, to relate 3D microscopy imaging data to non-invasive in vivo functional imaging methods with lower resolutions, a scalable microscopy method is needed that allows for large fields of view (FOVs) approaching those of the functional methods.

Here we present angioMASH: a pipeline for clearing and double staining angioarchitecture and cytoarchitecture in large cleared human samples. Our new protocol combines the scalable, economic cytoarchitecture staining as well as the effective clearing of $\mathrm{MASH}^{30}$ with protocols for labelling vasculature. The entire pipeline takes 
approx. three weeks to complete from slicing the sample to microscopy. We use a combination of MASH, the SWITCH labelling approach $^{36}$ and the protocol published by Harrison et al. ${ }^{42}$ to stain cortical angioarchitecture down to the capillary level in large human brain samples. We set out to investigate, whether angioMASH can visualise known vascular features such as the proposed layers of Duvernoy ${ }^{5}$ and then go beyond those descriptive results by quantitatively investigating vascular orientation and distributions. We then compared the vasculature between area V1 and V2 and show marked differences in their density and distribution over the cortical depth. 


\section{Materials and Methods}

\section{Human brain tissue}

Brain tissue samples were taken from 3 different healthy human body donors (see supplementary table 1 for donor data) of the body donation program of the Department of Anatomy and Embryology, Maastricht University. The tissue donors gave their informed and written consent to the donation of their body for teaching and research purposes as regulated by the Dutch law for the use of human remains for scientific research and education ("Wet op de Lijkbezorging"). Accordingly, a handwritten and signed codicil from the donor posed when still alive and well, is kept at the Department of Anatomy and Embryology Faculty of Health, Medicine and Life Sciences, Maastricht University, Maastricht, The Netherlands.

Brains were first fixed in situ by full body perfusion via the femoral artery. Under a pressure of 0.2 bar, the body was perfused by 101 fixation fluid (1.8 vol \% formaldehyde, $20 \%$ ethanol, $8.4 \%$ glycerine in water) within 1.5-2 hours. Thereafter the body was preserved at least 4 weeks submersed in the same fluid for post-fixation. Subsequently, brains were recovered by calvarial dissection and stored in $4 \%$ paraformaldehyde in $0.1 \mathrm{M}$ phosphate buffered saline (PBS) for 14-30 months. 


\section{Clearing and labelling of human brain samples}

Each of the occipital lobes was cut into approx. $3 \mathrm{~mm}$ thick coronal slices. Of each of the three occipital lobes, three consecutive slices were further blocked into pieces of about 3x2 cm (see Suppl. Fig. 1-3).

Tissue blocks were then processed with angioMASH, an adapted version of the MASH protocol ${ }^{30}$. We combined the lectin labelling from Harrison et al. ${ }^{42}$ with the SWITCH labelling strategy ${ }^{36}$ and MASH. The combination with SWITCH and performing sample delipidation before staining, rather than at the end, were used to improve label penetration in very large, thick human brain samples. The samples were kept on a shaker during incubation at all times. They were placed in 6 well cell culture plates and dehydrated $1 \mathrm{~h}$ each in $5 \mathrm{ml} 20,40,60$, $80,100 \%$ methanol $(\mathrm{MeOH}) / \mathrm{H}_{2} \mathrm{O}(\mathrm{v} / \mathrm{v})$ at room temperature $(\mathrm{RT})$ and 1h in $100 \% \mathrm{MeOH}$ at $4{ }^{\circ} \mathrm{C}$. Samples were then bleached overnight in freshly prepared, chilled $5 \% \mathrm{H}_{2} \mathrm{O}_{2}$ in $\mathrm{MeOH}(\mathrm{v} / \mathrm{v})$ at $4{ }^{\circ} \mathrm{C}$. This was followed by a delipidation step. For this, samples were placed in $50 \mathrm{ml}$ tubes made of polypropylene as cell culture plates are incompatible with dichloromethane (DCM). Samples were incubated for $8 \mathrm{~h}$ in $50 \mathrm{ml}$ of $66 \%$ DCM/33\% MeOH and 2x 1h in 100\% DCM at RT. Samples were then stored overnight in $80 \% \mathrm{MeOH}$ and rehydrated the next day in 5 $\mathrm{ml}$ each of $60,40,20 \% \mathrm{MeOH} / \mathrm{H}_{2} \mathrm{O}(\mathrm{v} / \mathrm{v})$ at RT in 6 well cell culture plates for $1 \mathrm{~h}$ respectively. This was followed by a second bleaching step in $50 \%(\mathrm{w} / \mathrm{v})$ aqueous potassium disulfite solution for $1 \mathrm{~h}$ at RT. The solution was stirred at approx. $70^{\circ} \mathrm{C}$ until all precipitated crystals 
were dissolved and filtered right before use. The solution can be reused several times. Samples were thoroughly rinsed $5 \mathrm{x}$ in distilled water and washed for another hour in distilled water at RT. Finally, samples were permeabilized $2 \times 1 \mathrm{~h}$ in $0.2 \%$ PBST (phosphate buffered saline $+0.2 \%(\mathrm{v} / \mathrm{v})$ Triton $\mathrm{x}-100$ adjusted to $\mathrm{pH} 7.4)$ and equilibrated overnight in SWITCH-OFF buffer (10 mM SDS in PBS at pH 7.4).

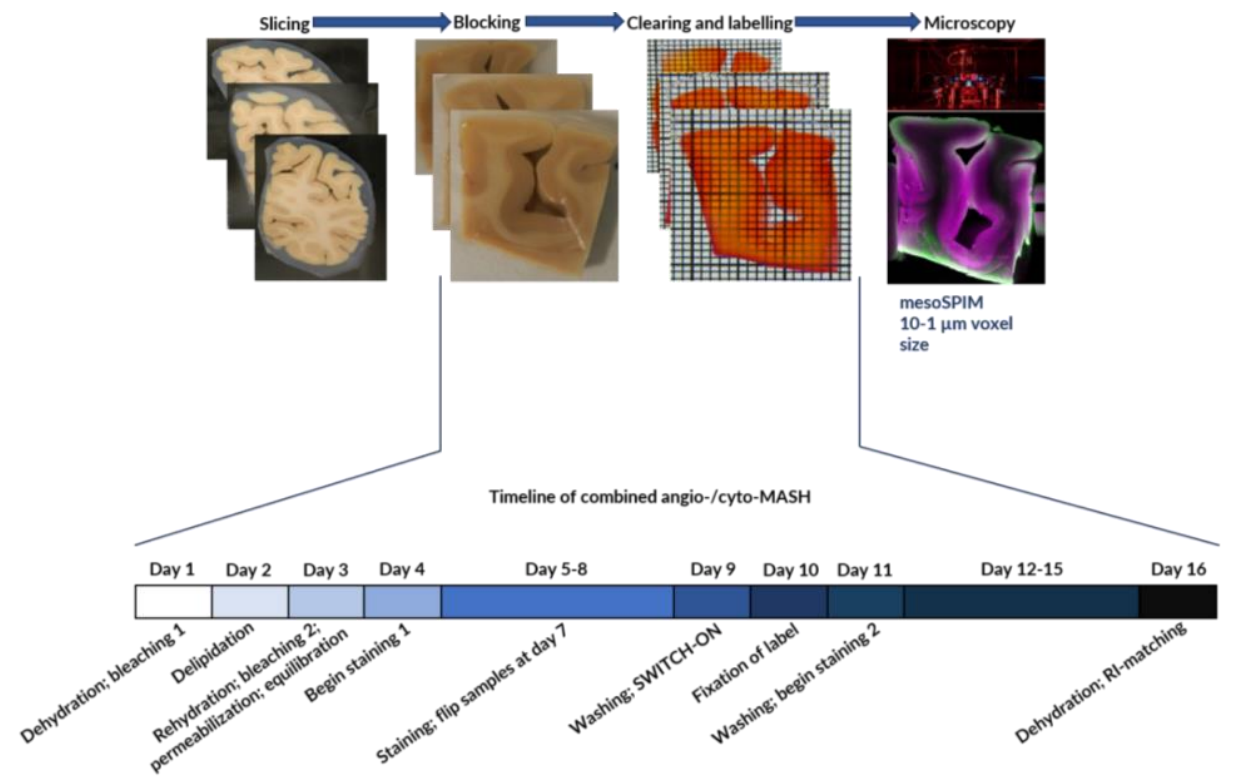

Figure 1: Workflow of the combined angio-/cytoMASH pipeline. Top shows representative examples of the tissue at all major steps of the pipeline, from cutting coronal sections of the agar-embedded occipital lobes and blocking ROls around the calcarine sulcus, to the clearing and imaging. The detailed timeline for the clearing and labelling of the tissue is given below.

Samples were stained for angioarchitecture in SWITCH-OFF buffer containing Lycopersicon esculentum lectin conjugated to 
DyLight@649 (Vector Laboratories, Inc.) diluted 1:100. Samples were incubated for 5 days and flipped after half the incubation time. Samples were then washed $3 \mathrm{x} 1 \mathrm{~h}$ in $5 \mathrm{ml} 0.2 \%$ PBST, placed again in $50 \mathrm{ml}$ tubes and incubated for $24 \mathrm{~h}$ in the same solution (referred to as the SWITCH-ON step). Subsequently, samples were incubated $24 \mathrm{~h}$ in $4 \%$ PFA. This step has been empirically determined to improve the labelling quality. It is assumed that the lectins are fixed by the PFA. This was followed by $3 \mathrm{x} 1 \mathrm{~h}$ washed in Mcllvain buffer at $\mathrm{pH} 4$ (see McIlvain, 1921 for details) ${ }^{43}$. For MASH-NR counterstaining ${ }^{30}$, samples were placed again in 6 well cell culture plates and incubated in $5 \mathrm{ml}$ $0.001 \%$ neutral red solution in Mcllvain buffer at $\mathrm{pH} 4$ for 5 days at RT. Samples were flipped after half the incubation time. Finally, samples were washed again $2 \mathrm{x} 1 \mathrm{~h}$ in $5 \mathrm{ml}$ of Mcllvain at $\mathrm{pH} 4$ and dehydrated 1 h each in $5 \mathrm{ml} 20,40,60,80,2 \times 100 \% \mathrm{MeOH} / \mathrm{H}_{2} \mathrm{O}$ (v/v). To ensure that all $\mathrm{MeOH}$ is removed before refractive index matching, samples were placed once more in $50 \mathrm{ml}$ tubes and incubated $1 \mathrm{~h}$ in 66 $\% \mathrm{DCM} / 33 \% \mathrm{MeOH}$ and 100\% DCM respectively. Then samples were immersed in ethyl cinnamate (ECi). The entire workflow is depicted schematically in figure 1 . Note that small samples containing mainly grey matter can be immersed in ECi directly after the last incubation in $100 \% \mathrm{MeOH}$. However, in larger samples the white matter, especially the deep white matter, can remain slightly opaque. 


\section{Light-sheet microscopy}

The samples were imaged on mesoSPIM light-sheet microscopy systems $^{44}$. The microscope consists of a dual-sided excitation path using a fiber-coupled multiline laser combiner (405, 488, 515, 561, 594, $647 \mathrm{~nm}$, Omicron SOLE-6) and a detection path comprising an Olympus MVX-10 zoom macroscope with a $1 \times$ objective (Olympus MVPLAPO 1×), a filter wheel (Ludl 96A350), and a scientific CMOS (sCMOS) camera (Hamamatsu Orca Flash 4.0 V3). This set-up allows for nearly isotropic resolution by applying axial scanning in which the beam waist is scanned with electrically tunable lenses (ETL, Optotune EL-16-40-5D-TC-L) synchronized with the rolling shutter of the sCMOS camera. The cytoarchitecture label (neutral red) was excited with $561 \mathrm{~nm}$ and DyLight $囚 649$-conjugated lectin with $647 \mathrm{~nm}$. For both channels, a multiband emission filter (QuadLine Rejectionband ZET405/488/561/640, AHF) was used. We first acquired large FOV overview scans of all brain samples with dual sided illumination at a pixel size of $10.52 \mu \mathrm{m}$ and a step size of $10 \mu \mathrm{m}$. The cytoarchitecture staining had lower wavelength excitation and emission spectra and hence could not be imaged as deeply as the far-red vessel label. We therefore acquired slightly higher resolution $(6.55 \mu \mathrm{m}$ in plane and $6 \mu \mathrm{m} z$-step size) overviews with single sided excitation by tilting the sample $20^{\circ}$ with respect to the excitation light. In this orientation, the excitation light has to travel through less tissue when imaging the more medial parts of the sample and therefore, the illumination of the cytoarchitecture channel (shorter wavelength) is better. We then 
acquired tiled high-resolution (2.03 $\mu \mathrm{m}$ pixel size and $2 \mu \mathrm{m}$ z-step) data around the V1/V2 border, identified beforehand based on the macroanatomical landmark of the stripe of Gennari. Finally, for one sample, we cut off a small gyrus containing parts of V1 and V2 on each side of it and acquired separate data sets at the highest resolution the mesoSPIM allowed (1.03 $\mu \mathrm{m}$ pixel size with $1 \mu \mathrm{m}$ step size). We opted to cut off this part as the cortical wall was relatively straight and it allowed for an illumination perpendicular to the cortical layers, so that an illumination gradient over the cortical depth could be avoided.

\section{Data analysis and visualisation}

Image pre-processing was done in FIJI ${ }^{45}$. Overview images acquired with a $20^{\circ}$ tilt were resliced and rotated for better visualisation. Tiled high-resolution acquisitions were stitched using the BigStitcher plugin $^{46}$. The vessel segmentation and skeletonisation was carried out in Vision4D (v3.4, arivis AG, Munich, Germany). To this end, highresolution raw datasets were first filtered with a 3D Laplacian of Gaussian filter using a FIJI plugin ${ }^{47}$. Data was separately filtered with a sigma of 2 and 4 pixels in each direction, to better preserve small vessels in the channel with the smaller filter setting and larger blood vessels in the channel with the larger filter setting. Binary masks of these two channels were created in FIJI and imported into Vision4D, where the two binary datasets were added (AND) into a single channel. The resulting combined binary channel was used as the image source for the skeletonisation into a new channel containing single pixel 
representations of the vessel centrelines. Skeletonisation was performed with a custom-made python script executed within the Vision4D environment (see Suppl. Fig. 4 for a description of the skeletonisation workflow), kindly provided by Maurizio Abbate (senior application support engineer \& image analysis specialist arivis AG, Munich, Germany).

For orientation analysis of the skeleton, 10 maximum intensity projections (MIPs) over 100 planes $(100 \mu \mathrm{m})$ were created for every successive $100 \mu \mathrm{m}$ of the $1 \mathrm{~mm}$ thick stack. The analysis was performed in cortical sub-compartments, by selecting 200x200 pixel regions of interest (ROIs) along the vertical centre line of each MIP. The ROIs were placed into upper layer III (V1 and V2 dataset, referred to as supragranular), layer IVc (V1) and layer IV (V2, referred to as granular respectively), and layers V/VI (V1 and V2, referred to as infragranular). Orientations of the skeleton channel were generated using the OrientationJ plugin for $\mathrm{FIJI}^{48,49}$.

For density analysis, the combined binary channel was cropped into a 100 pixel wide column covering the entire vertical length of the image. The column was manually placed into a region in which the layers were oriented as perpendicular towards it as possible in the slightly curved datasets. Of this cropped stack, 10 consecutive MIPs were created, each over 100 planes $(100 \mu \mathrm{m})$ and vertical profiles of average pixel intensities were generated with FIJI. 
Both the orientation distribution for each MIP and each of the ROIs, as well as the average pixel intensities for all columns created for the density analysis, were exported as spreadsheets into Microsoft Excel 2016 (Microsoft Corporation, Redmond, US) for further analysis. 


\section{Results}

The angioMASH approach (Fig. 1) allows for a deep penetration of the lectin label over several millimetres of tissue (Fig. 2 and Suppl. Video 1). Even at relatively low resolutions, marked changes in the angioarchitecture between V1 and V2 are visible, in particular the vessel dense stripe at layer IVb-c in V1 (see arrows Fig. 2). This matches the description by earlier authors ${ }^{5,27}$.

Multi-scale data sets were acquired from an isotropic resolution of $\sim 10 \mu \mathrm{m}$ up to $2 \mu \mathrm{m}$ for all the samples. A representative example of the different resolution levels is shown in figure 3 . The light scattering in large FOV overview scans is very strong towards the medial sample regions, especially in the lower wavelength cytoarchitecture staining (Fig. 3 a: excitation light coming from the top and bottom of the panel). Therefore, slightly higher resolution overviews where performed, in which the cytoarchitecture staining could be better appreciated (Fig. $3 \mathrm{~b}$ ). In these acquisitions, the sample was rotated $20^{\circ}$ oblique to the direction of the exciting light. For each sample, multiple tiles were acquired at high-resolution covering an extensive area around the V1/V2 border (Fig. $3 \mathrm{c}$ and Suppl. Video 2). Figure $3 \mathrm{~d}$ shows a zoomin of the highest acquired resolution of $2 \mu \mathrm{m}$, illustrating qualitatively the capacity to distinguish microscopic elements of both cyto- and angio-architecture. 


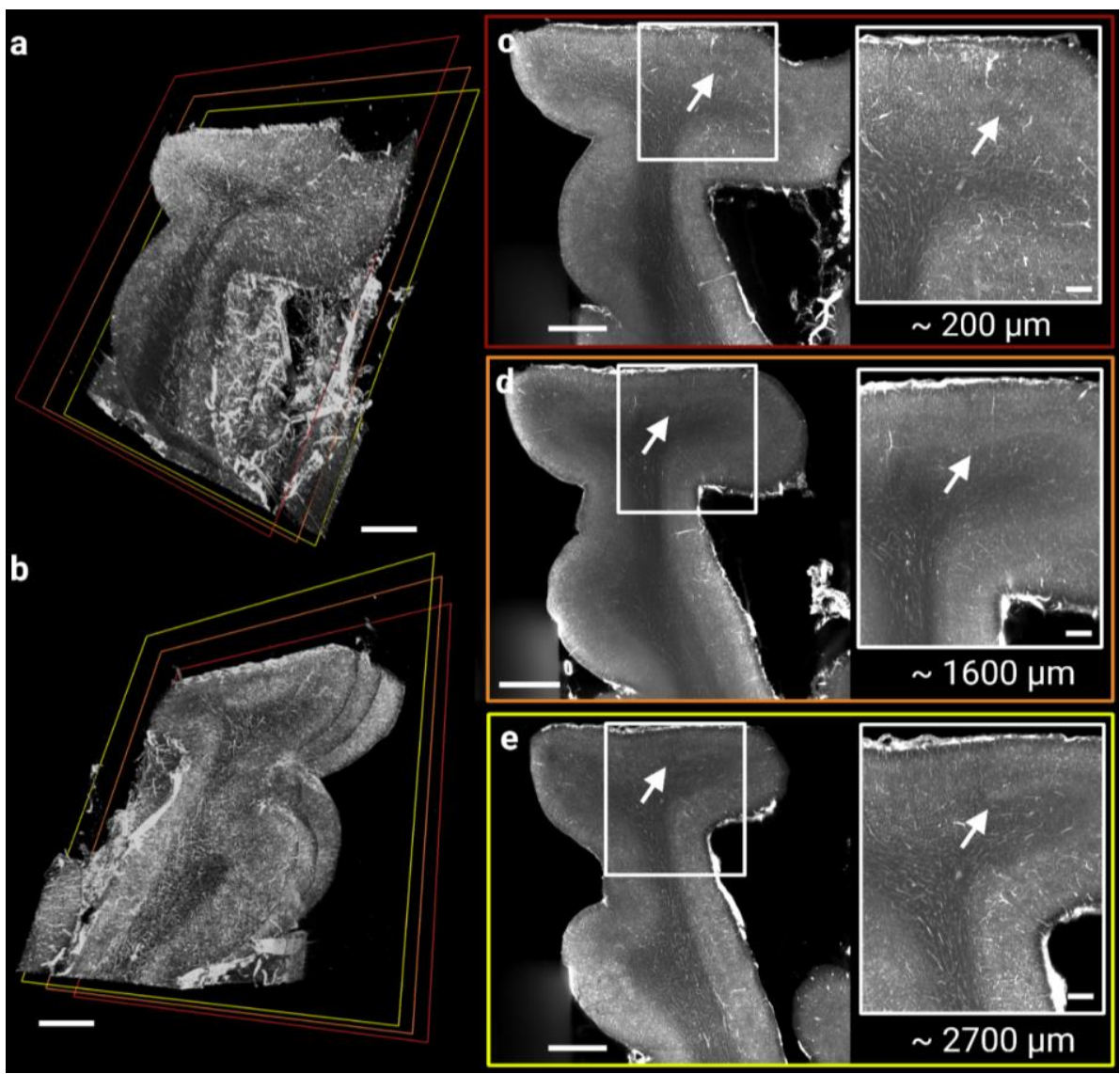

Figure 2: Overview acquisitions of the samples illustrate the quality and depth penetration of the vasculature staining. 3D rendering of a sample taken from Occipital lobe 3 showing the posterior (a) and anterior (b) side. The frames indicate the approximate positions of the MIPs shown in c-e. Panels c-e were projected over $150 \mu \mathrm{m}$ located at the posterior side (c; 200 $\mu \mathrm{m}$ deep into the tissue), middle (d; $1600 \mu \mathrm{m}$ deep into the tissue), and anterior side (e; $2700 \mu \mathrm{m}$ deep into the tissue) of the sample respectively. The vascular label is clearly visible throughout the whole sample thickness with a particularly dense stripe demarcating V1 (white arrows). Scale bars: $2 \mathrm{~mm}$ for each full view and $500 \mu \mathrm{m}$ for each magnified panel. 


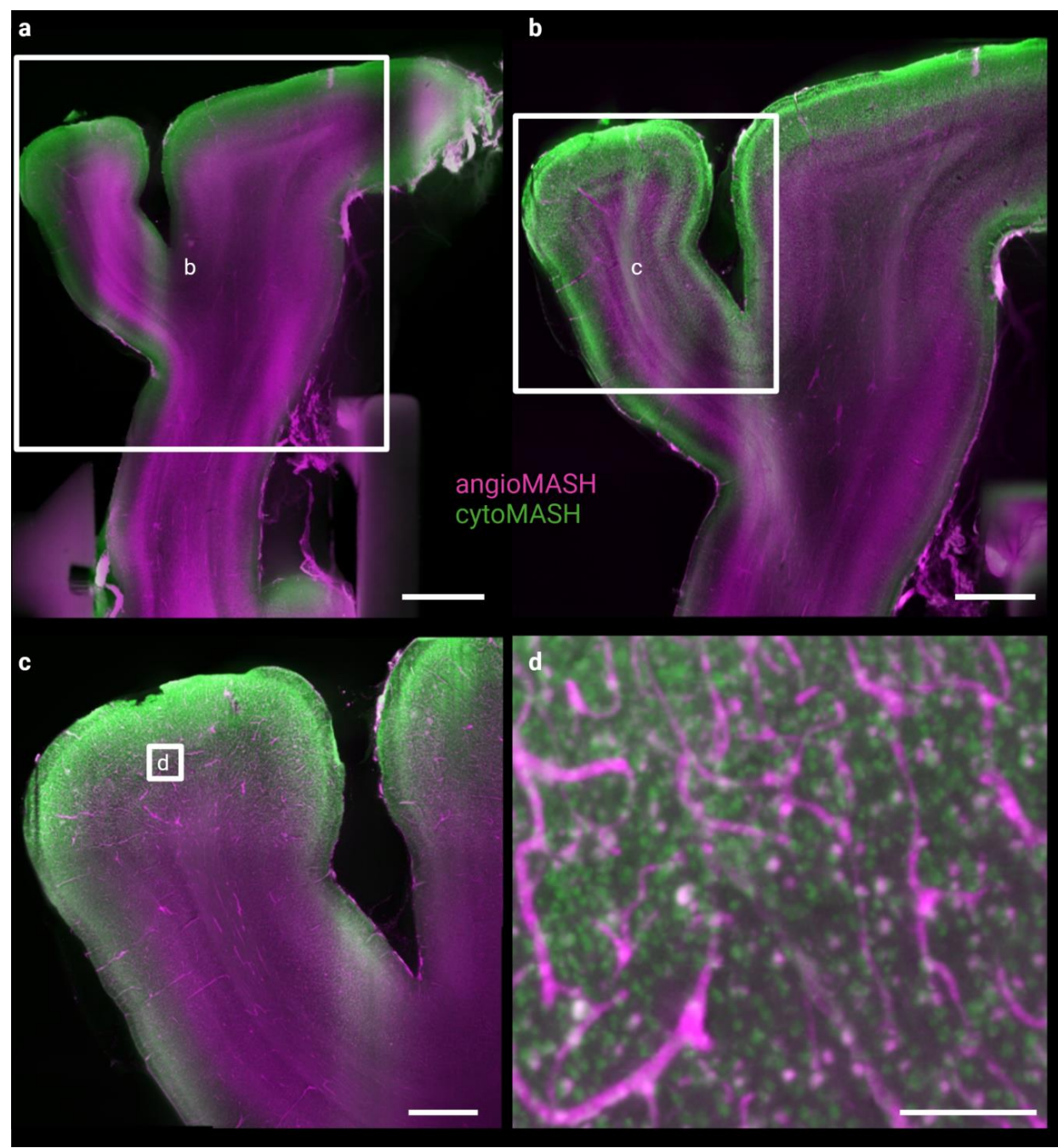

Figure 3: Representative example of the multi-scale data acquired for all specimen showing the cytoarchitecture stain in green and the vessel label in magenta. a) We first acquired fast overview scans with the highest FOV possible and a voxel size of about $10 \mu \mathrm{m}$ (MIP over $50 \mu \mathrm{m}$; scale bar: $4 \mathrm{~mm}$ ). Legend continued on next page. 
Figure 3: Continued. b) Light scattering is especially prominent in very large tissue pieces combined with a classical light-sheet geometry, in which the excitation light as to travel through the entire lateral length of the sample. We therefore performed overview acquisitions at slightly higher resolution $(6 \mu \mathrm{m})$ as well, in which the sample was rotated $20^{\circ}$ (MIP over $60 \mu \mathrm{m}$; scale bar: 2 $\mathrm{mm})$. c) Tiled and stitched high-resolution dataset of the ROI around the V1/V2 border (MIP over $50 \mu \mathrm{m}$; scale bar: $1 \mathrm{~mm}$ ). d) Magnified region indicated in panel $\mathbf{c}$, demonstrating the resolution down to the capillary and cell level (scale bar: $100 \mu \mathrm{m}$ )

It can already be appreciated that the choice of colour channels, i.e. longer more deeply penetrating wavelength for the angio-channel and shorter less deeply penetrating (and therefore more scattering) wavelength for the cyto-channel, was chosen to maximize imaging quality for the angio-architecture. In these datasets, cytoarchitectural features and capillaries can be clearly distinguished.

A qualitative comparison of the angioarchitecture between V1 and V2 in relation to the cytoarchitectural layers reveals striking differences, both in the overall density as well as the distribution of the vasculature (Fig. 4). For best visualisation of both features, the MIPs for the angioand cyto-channels were projected over a different depth. Angioarchitecture was judged to be best visible at projections over at least $100 \mu \mathrm{m}$, whereas cytoarchitecture was not ideally visible in such thick projections. We opted therefore for $150 \mu \mathrm{m}$ MIPs for angioarchitecture and $30 \mu \mathrm{m}$ MIPs, taken from the middle, for 
cytoarchitecture. For this comparison, the highest resolution data (at $1 \mu \mathrm{m}$ isotropic) was used as it illuminated the cortical wall perpendicular to the layers, which allowed for better inspection of the architecture. As seen in the higher FOV datasets, the shorter wavelength of the excitation light in the cyto-channel leads to more light-scattering and hence a gradient in the image from left to right. In V1, the vascular layers appear more pronounced in general. Blood vessels are mainly radially organized in supragranular layers there and many larger vessels terminate in layer III. The orientation of the blood vessels becomes more diverse in layers IVa-b, and a particularly dense mid-cortical vascular layer is visible in the lower part of layer IVb and within layer IVc. The main vessel orientation changes abruptly in the infragranular layers, where they run mainly tangentially. The border between grey and white matter is clearly visibly by a sharp decline in vascular density, while retaining their mainly tangential orientation. In both V1 and V2, two distinct vascular sub-layers are visible within cytoarchitectural layer I: The first sub-layer is almost devoid of small capillaries while the second one shows a marked increase in vessel density, caused mainly by smaller vessels. In V2, we were not able to determine a visible difference between Duvernoy's layer Ib and II. In general, the layers were less striking in this area. The border between layers II and III is marked by the termination of many smaller radial vessels, although this border is much less well visible as compared to $\mathrm{V} 1$ and the delineation much more tentative. 


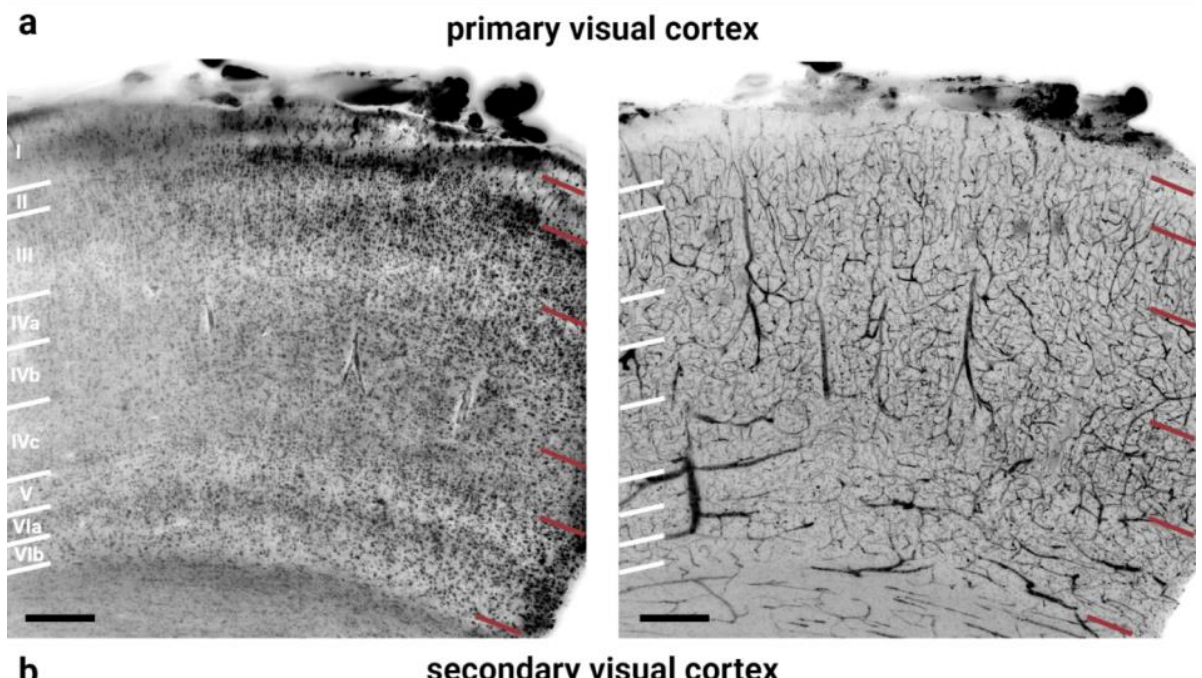

b

secondary visual cortex
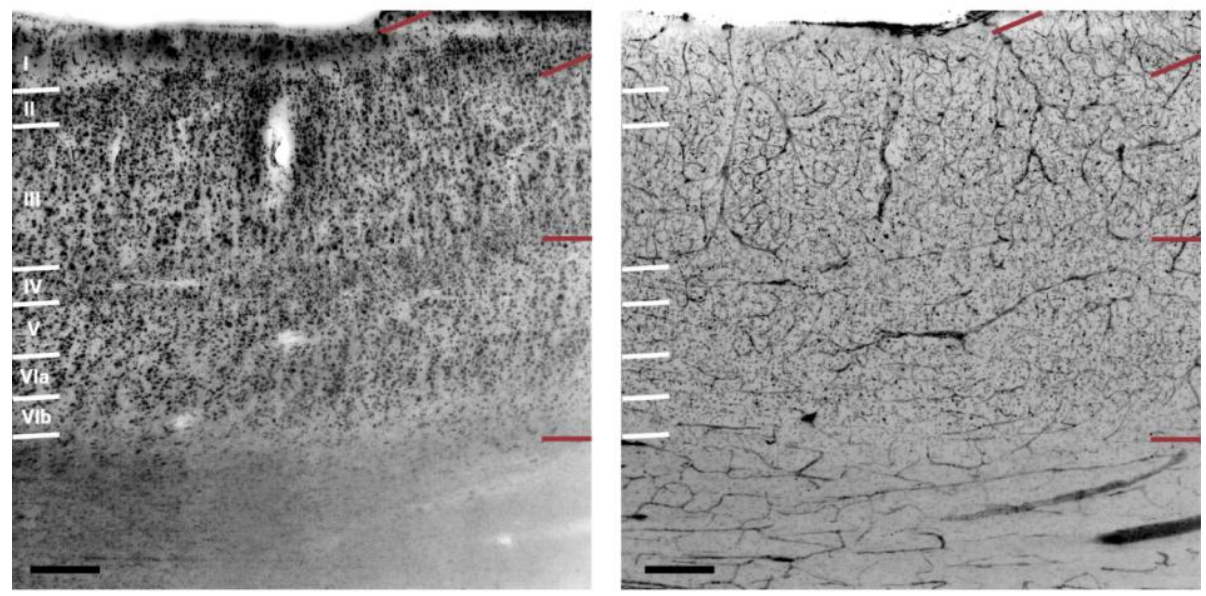

Figure 4: Joint visualisation of angio- and cytoarchitectural layers of V1 and V2. a) Cytoarchitecture (left) and angioarchitecture (right) of primary visual cortex (V1). b) Cytoarchitecture (left) and angioarchitecture (right) of secondary visual cortex (V2). Layers for angioarchitecture were based on the description of the 4 principle layers according to Duvernoy ${ }^{4}$ (red labels). Legend continued on next page. 
Figure 4: Continued. The layers as identified by cytoarchitecture (white labels, based on the description by von Economo and Koskinas ${ }^{1}$ ) are indicated in the angioarchitecture panels as well and vice versa. The MIPs were projected over $150 \mu \mathrm{m}$ MIPs for angioarchitecture and $30 \mu \mathrm{m}$ MIPs, taken from the middle, for cytoarchitecture. Scale bars: $250 \mu \mathrm{m}$ respectively.

The seemingly densest layer, layer III extends roughly to the borders of the cytoarchitecturally defined layer IV. After that, vessel density decreases and the general orientation of the vasculature seems again to be predominantly tangential. 
a
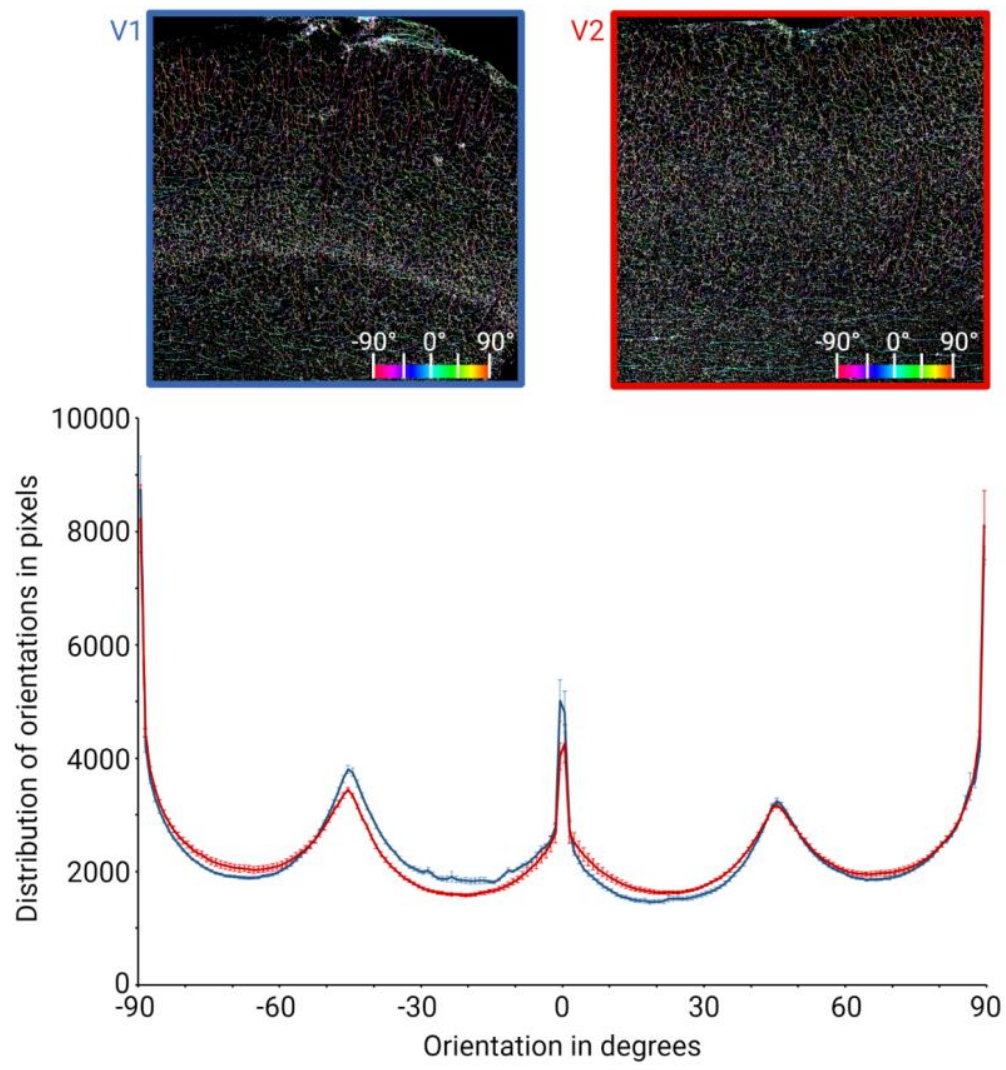

b
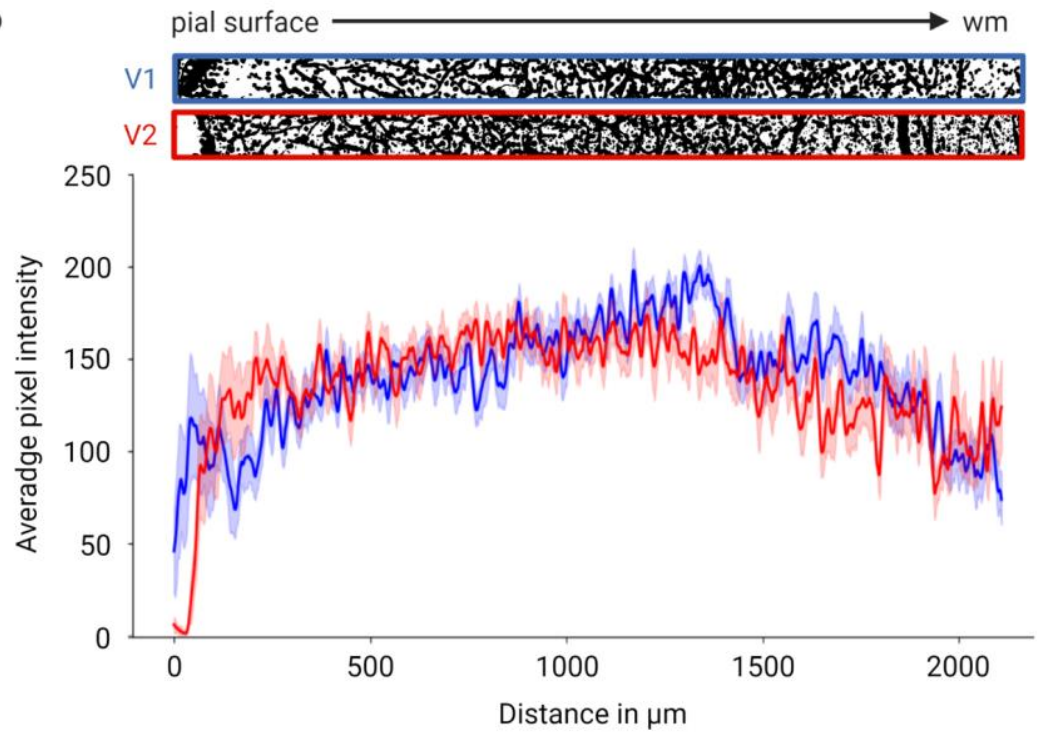
Figure 5: Comparison of vascular features between V1 and V2 (prior page). a) Averaged distribution of orientations generated from the vascular skeleton of V1 (blue) and V2 (red). Distribution of orientations as measured with OrientationJ (add ref), were generated over the entire FOV and averaged over the 10 consecutive MIPs. Colour coded maps of representative examples of MIPs taken from the middle of the $1 \mathrm{~mm}$ thick stack are shown below the graph. The vertical orientation is represented in red $\left(90^{\circ}\right.$ and $-90^{\circ}$ respectively), and the horizontal orientation in cyan $\left(0^{\circ}\right)$. (error bars: SEM). b) Comparison of vascular density over cortical depth between V1 (blue) and V2 (red). Pixel intensities averaged over the 100 pixel width of a column extending through the entire vertical length of the image with $0 \mu \mathrm{m}$ corresponding with the top side of the image and the pial surface direction, and $2100 \mu \mathrm{m}$ corresponding with the bottom of the image directed towards the white matter. Dark red and blue curves represent the smoothed mean values, lighter shaded bands the SEM. Representative examples of comlumns in the same orientation are given above the graph.

Regarding vessel orientation, the overall distribution of orientations in V1 and V2 appears very similar (Fig. 5 a). Both show dominant orientation in the radial and tangential direction of the cortex, as expected, although there are clear peaks in the distribution of orientations at $-45^{\circ}$ (lower left to upper right image diagonal) and $45^{\circ}$ (upper left to lower right diagonal of the image). These peaks can only partially be accounted for by the respective cortical curvature of both datasets. Density analysis of the average vasculature in V1 and V2 confirmed and quantified several features observed in the qualitative 
description (Fig. 5a). Both areas show an increase in vascular density towards the granular layers. As shown above in figure $4 \mathrm{a}$, V1 has a marked peak around $1300 \mu \mathrm{m}$ into the cortex corresponding roughly to layer IVc. There is no distinct difference in the density of (lower) layer III and IV in V2. In both areas the vascular density decreases in the infragranular layers and towards the white matter, although this decrease appears more continuous and less abrupt compared to the qualitative comparison. Surprisingly, the more superficial layers in V2 seem to have a higher density than $\mathrm{V} 1$, which is not readily apparent in figure 4.

To investigate the distributions of vessel orientations more closely, an orientation analysis was performed for each area to disentangle orientation in supragranular, granular, and infragranular layers. As expected from the earlier descriptions of Duvernoy ${ }^{5}$, the radial vessel orientation is especially pronounced in the supragranular layers of V1 (Fig. 6). As the vessel density increases (compare Fig. 5 b), the vasculature becomes more evenly distributed. In infragranular layers more blood vessesl maintain a tangential orientation as expected, although this is less apparent in the distribution of orientations as compared to the visual inspection (compare Fig. 4). Overall, the vasculature in area V2 is distributed more evenly (Fig. 7). In the supragranular layers, there still is a noticeable tendency towards a more radial orientation, however not as pronounced as in V1. The distribution of vessel orientations in granular and infragranular layers of V2 is very similar and again the switch towards a more tangential 
orientation in the infragranular layers, though perceptible, is less pronounced than in V1. This means that while the general distribution of the vasculature over the entire cortex is very similar between both V1 and V2 (Fig. 5a), clear inter-and intraareal differences become apparent with a more fine-grained quantitative analysis. 

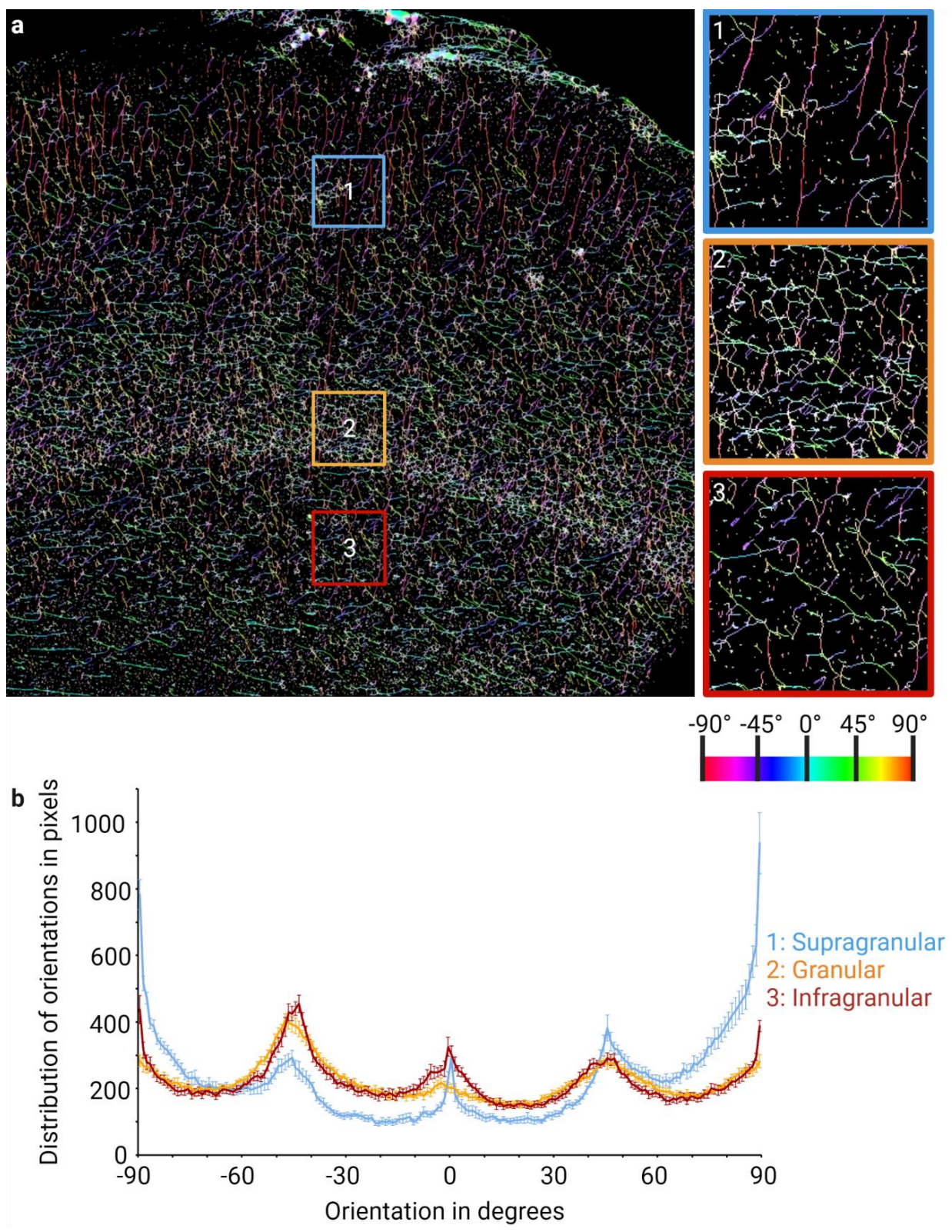

Figure 6: Main orientation of the vascular skeleton of V1. Legend continued on the next page. 
Figure 6: Continued. a) Colour coded orientation maps of a representative MIP taken from the middle of the $1 \mathrm{~mm}$ thick stack of the skeletonized angio channel, and indicated ROIs. The vertical (radial) orientation is represented in red $\left(90^{\circ}\right.$ and $-90^{\circ}$ respectively), and the horizontal (tangential) orientation in cyan $\left(0^{\circ}\right)$. b) Averaged distribution of the orientations for supragranular (blue), granular (orange), and infragranular (red) ROls (error bars: SEM). 

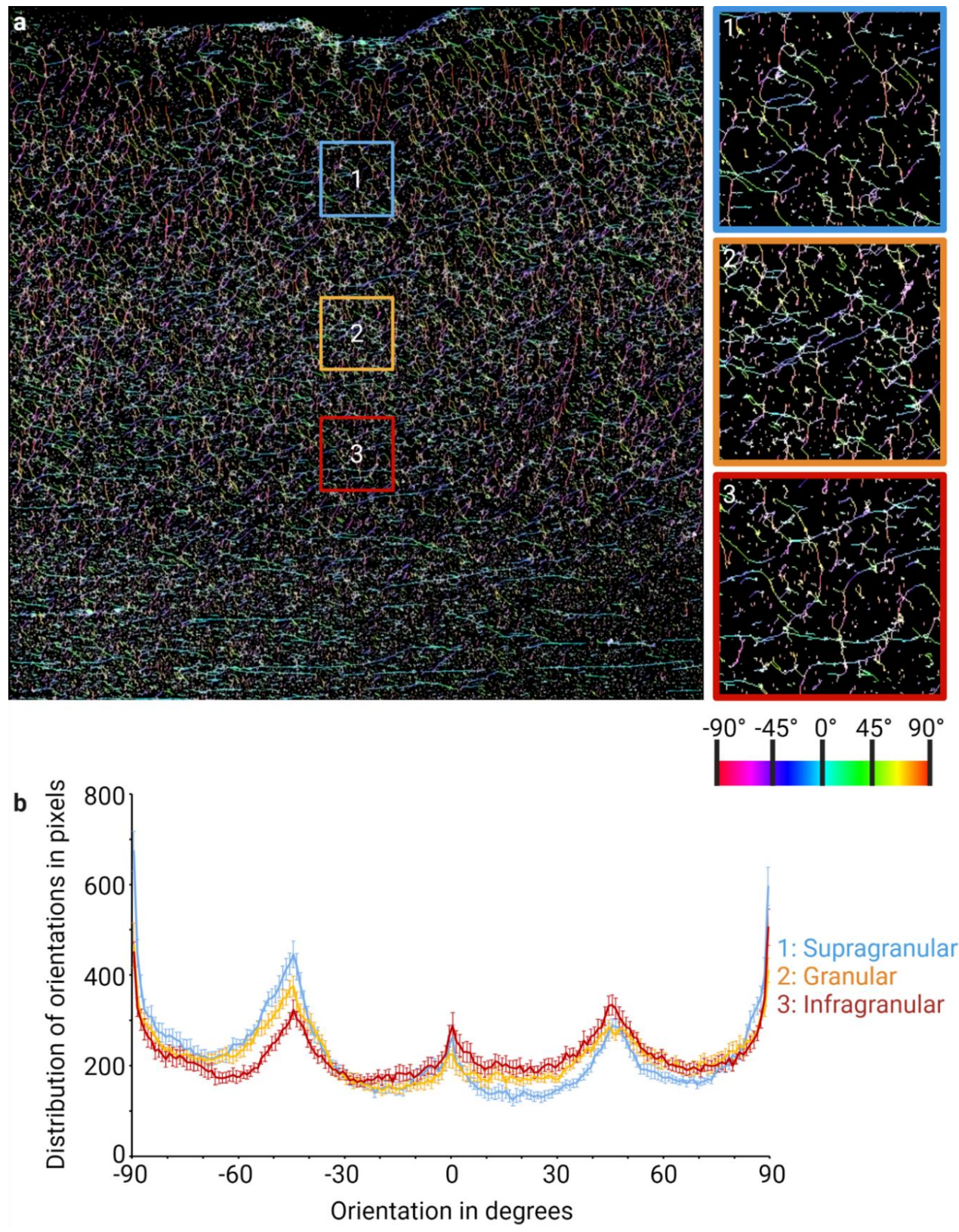

Figure 7: Main orientation of the vascular skeleton of V2. Legend continued on the next page. 
Figure 7: Continued. a) Colour coded orientation maps of a representative MIP taken from the middle of the $1 \mathrm{~mm}$ thick stack of the skeletonized angio channel, and indicated ROIs. The vertical (radial) orientation is represented in red ( $90^{\circ}$ and $-90^{\circ}$ respectively), and the horizontal (tangential) orientation in cyan $\left(0^{\circ}\right)$. b) Averaged distribution of the orientations for supragranular (blue), granular (orange), and infragranular (red) ROIs (error bars: SEM). 


\section{Discussion}

The angioMASH method presented here allows for the comprehensive study and mapping of the human angioarchitecture in 3D. We could show a noticeable difference in the vessel architecture between human areas V1 and V2. Although our work builds upon and extends the labelling protocol proposed by Harrison et al. ${ }^{42}$, in their work they only processed and imaged at capillary level in relatively small samples ( $2 \mathrm{~mm} \times 3 \mathrm{~mm} \times 3 \mathrm{~mm})$. Furthermore, their segmentation results show mainly larger vessels and a noticeable absence of small microvasculature and no analysis of any vascular features is shown. Similarly, a recent publication by the Ertürk group ${ }^{35}$ used lectin labels on much larger human brain samples, but shows no analysis of the angioarchitecture. In this study, we focussed on samples of an intermediate size ( $\sim 30 \mathrm{~mm} \times 10 \mathrm{~mm} \times 3 \mathrm{~mm})$, as the imaging of much thicker samples with light-sheet microscopy quickly becomes unrealistic. Yet, as no special equipment is needed for the clearing and labelling process, in principle much larger samples (laterally) could be processed with angioMASH. However, it is assumed unfeasible at this point, to extend the sample thickness beyond a few millimetres with the current imaging technology while keeping data quality good enough for the type of analyses presented here. As our tilted overview acquisitions showed, an oblique geometry of the light-sheet set-up ${ }^{50,51}$ would have the advantage of enabling a more homogenous imaging of very large, flat samples such as whole brain slices (see also Chapter 5). Light-sheet microscopy systems, which illuminate and image large 
flat samples from the same side would therefore be essential to upscale this pipeline as shown for the cytoMASH pipeline in other parts of this thesis (see Chapter 4).

In general, our findings match well with the descriptions of human and non-human primate visual cortex by other authors derived from 2D sections ${ }^{5,24,26}$, lending support to the high labelling quality achievable by the angioMASH technique. The density distribution found in V1 of the current samples appears to be highly similar as that in other primate species ${ }^{4,26}$, with a noticeable high density in layers IVb-c. In V2 our observed distribution matches the general description of Duvernoy ${ }^{5}$, who remarked that in contrast to other authors, his observations indicate that in most areas, lower cyto layer III shows at least as high a vascular density as cyto layer IV. In our data, the density of cyto layers III and IV were similarly identical. This contradicts Weber's observation in the macaque cortex of the highest density being located in layer $\mathrm{IV}^{26}$, and future investigation and analysis could determine if this finding is consistent and represents an inter-species difference. Likewise, it remains to be determined if other areas in the human brain are similarly different in their layered angio-organisation or whether the difference is mainly found in primary sensory versus other $\operatorname{areas}^{24}$.

Angioarchitecture is currently understudied in the human brain and of increasing importance for modern non-invasive imaging techniques whose signal is strongly coupled to the underlying 
vasculature, such as fNRIS ${ }^{14,15}$ and $\mathrm{fMRI}^{16,17}$. The data and results presented here could open the door towards a comprehensive ground truth investigation to validate and support the interpretation of these non-invasive imaging methods at a meso- and microscopic level. The inter- and intra-areal variability of cortical angioarchitecture and its relationship to cyto- and myeloarchitecture would be of particular interest. Already the investigation of both angio- and cytoarchitecture has been shown here to be possible and it is expected that the labelling and imaging routines will be scaled up in the near future to allow for the processing of much larger samples with angioMASH, as shown for (cyto)MASH in Chapter 4. Based on the preliminary results presented here, as well as data by other groups ${ }^{23,24,26}$, a direct one to one relationship between e.g. cyto- and angioarchitecture does not seem to exist. However, as the density analysis shows, there does seems to be a general trend of higher vascularisation in more metabolically demanding cortical regions (both within and between different areas). It seems therefore logical, that primary sensory cortices demonstrate their highest vasculature in the input layers, whereas other areas would show highest vascular density more in lower supragranular layers, assuming some association areas might have a dominance for internal computations to be more strongly represented here ${ }^{52}$. It would be intriguing to compare more areas and include higher areas as well as primary motor cortex to see if this simple model of vascular density following the metabolic demands of the respective cortical module holds true. 


\section{Technical considerations}

The tomato lectin applied here as a vessel label, binds to specific sugar-groups present in the epithelial cells of the vessel wall. Therefore, all the blood vessels, including the capillaries are labelled. However, a distinction between the different components of the vascular network into arteries, arterioles, veins, venules, and capillaries is not possible with this one marker alone. Particularly, vein and artery components cannot be separated, although arteries and veins, versus arterioles and venules, versus capillaries might be distinguished based on analysis of relative vessel diameters. Recently, the Renier laboratory published an outstanding atlas of whole mouse brain vasculature, based on several clearing compatible antibody labels to distinguish artery and vein components ${ }^{39}$. A different approach was taken by the Ertürk group, to use dye injections and machine learning to differentiate between the components in the cleared mouse brain ${ }^{41}$ and similar approaches have been used by other groups as well ${ }^{53,54}$. For future studies, the adaptation of one of the two approaches might allow a more detailed description of the vasculature in the human brain. If antibodies against vein- or artery-specific epitopes could be introduced deep enough into the much denser human brain tissue, the components could be separated into different channels.

A disadvantage of both lectin and antibody labels is that they only stain the vessel wall. Injection techniques have the advantage of filling the 
entire lumen, which should prevent larger vessels from collapsing during the clearing. This method would have the additional advantage of increasing the signal-to-noise ratio, making deep imaging easier ${ }^{53}$. A traditional approach to label angioarchitecture in the human brain, is the injection with Indian ink gel ${ }^{5,23,29}$. Adapting the classic Indian ink protocol towards a fluorescent, clearing compatible label, could allow for much better imaging deep into human brain samples and would allow for a more reliable determination of the diameters of larger vessels. One potential limitation of these injection based techniques, specifically in the human brain, are blood coagulations in the smallest vessels due to post mortem delays, which might lead to them being insufficiently labelled. Here the combination with angio-MASH could prove particularly useful.

Another limitation of the current study is that both density and orientation analysis, was carried out on multiple 2D MIPs rather than on the 3D volumes. In the future, a more sophisticated 3D analysis of the vascular network in the datasets acquired in this study could reveal more extensive spatial relationships. For instance, some orientations might be better represented in a 3D vascular skeleton, instead of the current collapsed 2D projection. A confounding factor in the direct comparison between $\mathrm{V} 1$ and $\mathrm{V} 2$ in the two datasets shown here is the different cortical curvature, which would influence the main vessel orientations when the radial orientation is not exactly parallel to the vertical axis of the images. However, it is assumed, that the dominant $45^{\circ}$ and $-45^{\circ}$ orientations arise at least in part from 
branches of larger penetrating vessels. One way to account for this bias could be the artificial straightening of the cortical ribbon. Another way could be to analyse 3D vasculature in a local cortical (tangentialradial) coordinate system. Crucially, future progress beyond the preliminary quantitative results presented here will include quantitative statistical tests for intra- and inter-areal differences in angio-architecture.

\section{Outlook}

The current datasets were acquired from the occipital lobes of healthy body donors. Two obvious next goals would be 1) the extension towards more brain areas, to get a more comprehensive overview of the healthy human angioarchitecture and 2) the comparison with pathological samples. Multiple brain disorders, such as vascular dementia $^{55}$ or epilepsy ${ }^{56,57}$ are known to result in abnormal vascular organisation or changes in the vessel anatomy itself. angioMASH could enable the study of these disorders in the human brain in it full 3dimensional aspects. Tissue clearing and light-sheet microscopy are already starting to be used for 3D investigation of healthy and pathological human brain anatomy ${ }^{32,33,35,58,59}$. Taking histological investigations into 3D can help highlight features, which would be difficult to detect on conventional 2D sections, as has been shown for the spatial distributions of plaques in Alzheimer's disease ${ }^{33}$. Although large scale brain mapping endeavours using $3 \mathrm{D}$ reconstructions of $2 \mathrm{D}$ sections have yielded astounding results ${ }^{60,61}$, the combination of tissue 
clearing and light-sheet microscopy has the potential to engage in similar brain mapping endeavours, with a view on how to achieve reference atlases with such quality with less person-years expended. Once scalable labelling techniques for other features of the human brain and the neocortex in particular are developed, this might enable the study of brain anatomy in an unprecedented manner. Currently, there are no reliable methods to label e.g. myelo-, glia-, or fibrilloarchitecture in thick, cleared human brain samples. However, we consider it only a matter of time until these methods are established. 


\section{References}

1 von Economo, C. \& Koskinas, G. N. Die Cytoarchitektonik der Hirnrinde des erwachsenen Menschen: Textband u. Atlas. (Springer, 1925).

2 Pfeifer, R. A. Die Angioarchitektonik der Grosshirnrinde. (J. Springer, 1928).

3 Pfeifer, R. A. Grundlegende Untersuchungen für die Angioarchitektonik des menschlichen Gehirns. (J. Springer, 1930).

4 Pfeifer, R. A. Die angioarchitektonische areale gliederung der grosshirnrinde: auf grund vollkommener gefässinjektionspräparate vom gehirn des macacus rhesus. (G. Thieme, 1940).

5 Duvernoy, H. M., Delon, S. \& Vannson, J. L. Cortical blood vessels of the human brain. Brain Research Bulletin 7, 519-579, doi:https://doi.org/10.1016/0361-9230(81)90007-1 (1981).

6 Brodmann, K. Vergleichende Lokalisationslehre der Grosshirnrinde in ihren Prinzipien dargestellt auf Grund des Zellenbaues. (Barth, 1909).

$7 \quad$ Hopf, A. Über die Verteilung myeloarchitektonischer Merkmale in der Stirnhirnrinde beim Menschen. J Hirnforsch 2, 311-333 (1956).

8 Nieuwenhuys, R. The myeloarchitectonic studies on the human cerebral cortex of the Vogt-Vogt school, and their significance for the interpretation of functional neuroimaging data. Brain structure \& function 218, 303-352, doi:10.1007/s00429-012-0460-z (2013).

9 Nieuwenhuys, R. \& Broere, C. A. J. A map of the human neocortex showing the estimated overall myelin content of the individual architectonic areas based on the studies of Adolf Hopf. Brain Structure and Function 222, 465-480, doi:10.1007/s00429-016-1228-7 (2017).

10 Nieuwenhuys, R. \& Broere, C. A. J. A detailed comparison of the cytoarchitectonic and myeloarchitectonic maps of the human neocortex produced by the Vogt-Vogt school. Brain structure \& function 225, 27172733, doi:10.1007/s00429-020-02150-2 (2020).

11 Vogt, C. \& Vogt, O. Allgemeine ergebnisse unserer hirnforschung. Vol. 21 (JA Barth, 1919). 
12 Vogt, C. \& Vogt, O. in Nervensystem 448-477 (Springer, 1928).

13 Vogt, O. Die myeloarchitektonische Felderung des menschlichen Stirnhirns. J Psychol Neurol 15, 221-232 (1910).

14 Kandice, T. et al. Spectrally resolved neurophotonics: a case report of hemodynamics and vascular components in the mammalian brain. Journal of biomedical optics 10, 1-9, doi:10.1117/1.2137291 (2005).

15 Jobsis, F. F. Noninvasive, infrared monitoring of cerebral and myocardial oxygen sufficiency and circulatory parameters. Science 198, 1264, doi:10.1126/science.929199 (1977).

16 Ogawa, S., Lee, T. M., Kay, A. R. \& Tank, D. W. Brain magnetic resonance imaging with contrast dependent on blood oxygenation. Proceedings of the National Academy of Sciences 87, 9868, doi:10.1073/pnas.87.24.9868 (1990).

17 Uludağ, K. \& Blinder, P. Linking brain vascular physiology to hemodynamic response in ultra-high field MRI. Neurolmage 168, 279-295, doi:https://doi.org/10.1016/j.neuroimage.2017.02.063 (2018).

18 Huber, L. et al. Layer-dependent functional connectivity methods. Progress in neurobiology, 101835, doi:https://doi.org/10.1016/j.pneurobio.2020.101835 (2020).

19 Huber, L. et al. High-Resolution CBV-fMRI Allows Mapping of Laminar Activity and Connectivity of Cortical Input and Output in Human M1. Neuron 96, 1253-1263 e1257, doi:10.1016/j.neuron.2017.11.005 (2017).

20 Huber, L., Uludağ, K. \& Möller, H. E. Non-BOLD contrast for laminar fMRI in humans: CBF, CBV, and CMRO2. Neurolmage 197, 742-760, doi:https://doi.org/10.1016/j.neuroimage.2017.07.041 (2019).

21 Kashyap, S. et al. Sub-millimetre resolution laminar fMRI using Arterial Spin Labelling in humans at $7 \mathrm{~T}$. PloS one 16, e0250504, doi:10.1371/journal.pone.0250504 (2021).

22 Schneider, M., Kemper, V. G., Emmerling, T. C., De Martino, F. \& Goebel, R. Columnar clusters in the human motion complex reflect consciously 
perceived motion axis. Proceedings of the National Academy of Sciences 116, 5096, doi:10.1073/pnas.1814504116 (2019).

23 Lauwers, F., Cassot, F., Lauwers-Cances, V., Puwanarajah, P. \& Duvernoy, $\mathrm{H}$. Morphometry of the human cerebral cortex microcirculation: General characteristics and space-related profiles. Neurolmage 39, 936-948, doi:https://doi.org/10.1016/j.neuroimage.2007.09.024 (2008).

24 Schmid, F., Barrett, M. J. P., Jenny, P. \& Weber, B. Vascular density and distribution in neocortex. Neurolmage 197, 792-805, doi:https://doi.org/10.1016/j.neuroimage.2017.06.046 (2019).

25 Tsai, P. S. et al. Correlations of Neuronal and Microvascular Densities in Murine Cortex Revealed by Direct Counting and Colocalization of Nuclei and Vessels. The Journal of Neuroscience 29, 14553, doi:10.1523/JNEUROSCI.3287-09.2009 (2009).

26 Weber, B., Keller, A. L., Reichold, J. \& Logothetis, N. K. The microvascular system of the striate and extrastriate visual cortex of the macaque. Cerebral cortex 18, 2318-2330, doi:10.1093/cercor/bhm259 (2008).

27 Adams, D. L., Piserchia, V., Economides, J. R. \& Horton, J. C. Vascular Supply of the Cerebral Cortex is Specialized for Cell Layers but Not Columns. Cerebral cortex 25, 3673-3681, doi:10.1093/cercor/bhu221 (2015).

28 de Crinis, M. in Anatomie der Sehrinde 33-36 (Springer, 1938).

29 Cassot, F., Lauwers, F., Fouard, C., Prohaska, S. \& Lauwers-Cances, V. A Novel Three-Dimensional Computer-Assisted Method for a Quantitative Study of Microvascular Networks of the Human Cerebral Cortex. Microcirculation 13 , 1-18, doi:https://doi.org/10.1080/10739680500383407 (2006).

30 Hildebrand, S., Schueth, A., Herrler, A., Galuske, R. \& Roebroeck, A. Scalable Labeling for Cytoarchitectonic Characterisation of Large Optically Cleared Human Neocortex Samples. Scientific reports 9, 10880, doi:10.1038/s41598-019-47336-9 (2019).

31 Hildebrand, S. et al. hFRUIT: An optimized agent for optical clearing of Dil-stained adult human brain tissue. Scientific reports 10, 9950, doi:10.1038/s41598-020-66999-3 (2020). 
32 Lai, H. M. et al. Next generation histology methods for three-dimensional imaging of fresh and archival human brain tissues. Nature communications 9, 1066, doi:10.1038/s41467-018-03359-w (2018).

33 Liebmann, T. et al. Three-Dimensional Study of Alzheimer's Disease Hallmarks Using the iDISCO Clearing Method. Cell reports 16, 1138-1152, doi:10.1016/j.celrep.2016.06.060 (2016).

34 Tainaka, K. et al. Chemical Landscape for Tissue Clearing Based on Hydrophilic Reagents. Cell reports 24, 2196-2210 e2199, doi:10.1016/j.celrep.2018.07.056 (2018).

35 Zhao, S. et al. Cellular and Molecular Probing of Intact Human Organs. Cell 180, 796-812 e719, doi:10.1016/j.cell.2020.01.030 (2020).

36 Murray, E. et al. Simple, Scalable Proteomic Imaging for High-Dimensional Profiling of Intact Systems. Cell 163, 1500-1514, doi:10.1016/j.cell.2015.11.025 (2015).

37 Yun, D. H. et al. Ultrafast immunostaining of organ-scale tissues for scalable proteomic phenotyping. bioRxiv, 660373, doi:10.1101/660373 (2019).

38 Di Giovanna, A. P. et al. Whole-Brain Vasculature Reconstruction at the Single Capillary Level. Scientific reports 8, 12573, doi:10.1038/s41598018-30533-3 (2018).

39 Kirst, C. et al. Mapping the Fine-Scale Organisation and Plasticity of the Brain Vasculature. Cell 180, 780-795 e725, doi:10.1016/j.cell.2020.01.028 (2020).

40 Lugo-Hernandez, E. et al. 3D visualisation and quantification of microvessels in the whole ischemic mouse brain using solvent-based clearing and light-sheet microscopy. Journal of cerebral blood flow and metabolism : official journal of the International Society of Cerebral Blood Flow and Metabolism 37, 3355-3367, doi:10.1177/0271678X17698970 (2017).

41 Todorov, M. I. et al. Machine learning analysis of whole mouse brain vasculature. Nature methods 17, 442-449, doi:10.1038/s41592-0200792-1 (2020). 
42 Harrison, C. H. et al. A novel method to visualise the three-dimensional organisation of the human cerebral cortical vasculature. Journal of anatomy 232, 1025-1030, doi:10.1111/joa.12805 (2018).

43 Mcllvaine, T. C. A buffer solution for colourimetric comparison. Journal of Biological Chemistry 49, 183-186 (1921).

44 Voigt, F. F. et al. The mesoSPIM initiative: open-source light-sheet microscopes for imaging cleared tissue. Nature methods 16, 1105-1108, doi:10.1038/s41592-019-0554-0 (2019).

45 Schindelin, J. et al. Fiji: an open-source platform for biological-image analysis. Nature methods 9, 676-682, doi:10.1038/nmeth.2019 (2012).

46 Horl, D. et al. BigStitcher: reconstructing high-resolution image datasets of cleared and expanded samples. Nature methods 16, 870-874, doi:10.1038/s41592-019-0501-0 (2019).

47 Sage, D., Neumann, F. R., Hediger, F., Gasser, S. M. \& Unser, M. Automatic tracking of individual fluorescence particles: application to the study of chromosome dynamics. IEEE Transactions on Image Processing 14, 13721383, doi:10.1109/TIP.2005.852787 (2005).

48 Rezakhaniha, R. et al. Experimental investigation of collagen waviness and orientation in the arterial adventitia using confocal laser scanning microscopy. Biomechanics and Modeling in Mechanobiology 11, 461-473, doi:10.1007/s10237-011-0325-z (2012).

49 Püspöki, Z., Storath, M., Sage, D. \& Unser, M. in Focus on Bio-Image Informatics (eds Winnok H. De Vos, Sebastian Munck, \& Jean-Pierre Timmermans) 69-93 (Springer International Publishing, 2016).

50 Glaser, A. K. et al. Light-sheet microscopy for slide-free non-destructive pathology of large clinical specimens. Nature biomedical engineering 1, doi:10.1038/s41551-017-0084 (2017).

51 Glaser, A. K. et al. Multi-immersion open-top light-sheet microscope for high-throughput imaging of cleared tissues. Nature communications 10 , 2781, doi:10.1038/s41467-019-10534-0 (2019). 
52 Harris, K. D. \& Shepherd, G. M. G. The neocortical circuit: themes and variations. Nature neuroscience 18, 170-181, doi:10.1038/nn.3917 (2015).

53 Di Giovanna, A. P. et al. Whole-Brain Vasculature Reconstruction at the Single Capillary Level. Scientific reports 8, 12573, doi:10.1038/s41598018-30533-3 (2018).

54 Henriksen, B. L. E., Jensen, K. H. R. \& Berg, R. W. Vasculature-staining with lipophilic dyes in tissue-cleared brains assessed by deep learning. bioRxiv, 2020.2005.2016.099705, doi:10.1101/2020.05.16.099705 (2020).

55 Nyenhuis, D. L. \& Gorelick, P. B. Vascular Dementia: A Contemporary Review of Epidemiology, Diagnosis, Prevention, and Treatment. Journal of the American Geriatrics Society 46, 1437-1448, doi:https://doi.org/10.1111/j.1532-5415.1998.tb06015.x (1998).

56 Löscher, W. \& Friedman, A. Structural, Molecular, and Functional Alterations of the Blood-Brain Barrier during Epileptogenesis and Epilepsy: A Cause, Consequence, or Both? International journal of molecular sciences 21, doi:10.3390/ijms21020591 (2020).

57 Gorter, J. A., van Vliet, E. A. \& Aronica, E. Status epilepticus, blood-brain barrier disruption, inflammation, and epileptogenesis. Epilepsy \& Behavior 49, 13-16, doi:https://doi.org/10.1016/j.yebeh.2015.04.047 (2015).

58 Liu, A. K. et al. Bringing CLARITY to the human brain: visualisation of Lewy pathology in three dimensions. Neuropathology and applied neurobiology 42, 573-587, doi:10.1111/nan.12293 (2016).

59 Liu, A. K. L., Lai, H. M., Chang, R. C. C. \& Gentleman, S. M. Free of acrylamide sodium dodecyl sulphate (SDS)-based tissue clearing (FASTClear): a novel protocol of tissue clearing for three-dimensional visualisation of human brain tissues. Neuropathology and applied neurobiology 43, 346-351, doi:https://doi.org/10.1111/nan.12361 (2017).

60 Amunts, K. et al. BigBrain: An Ultrahigh-Resolution 3D Human Brain Model. Science 340, 1472, doi:10.1126/science.1235381 (2013). 
61 Amunts, K., Mohlberg, H., Bludau, S. \& Zilles, K. Julich-Brain: A 3D probabilistic atlas of the human brain's cytoarchitecture. Science 369,988 , doi:10.1126/science.abb4588 (2020). 


\section{Appendix Chapter 3}

Supplementary Table 1: Patient information of the occipital lobe specimen.

Sample

Occipital lobe 1

\section{Gender Age}

male

82

Prior neuropathological disease
Occipital lobe 2 female 101

Occipital lobe 3 male 90 no

no

no

Supplementary Video 1. 3D volume rendering of an overview acquisition of occipital lobe 3 , sample 2 . The rendering shows the penetration of the vessel label throughout the sample (resolution $\sim 6 \mu \mathrm{m}$ ).

Supplementary Video 2. 3D volume rendering of the stitched high-resolution $(\sim 2 \mu \mathrm{m})$ data of occipital lobe 3 , sample 2 , showing the extent of the acquired tiled ROI. 

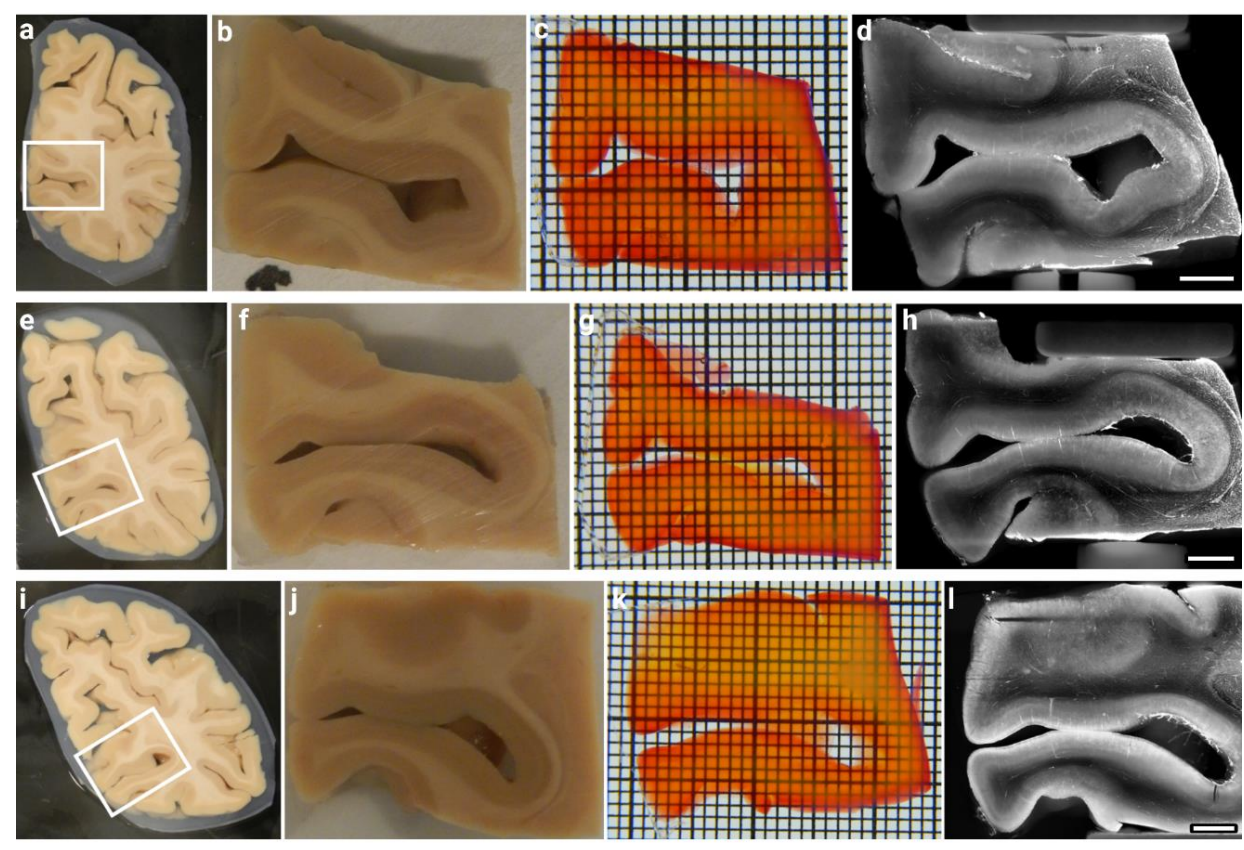

Supplementary Figure 1: Overview of Occipital lobe 1 samples. Three consecutive coronal slices (a, e, and I; direction posterior to anterior) where cut at a thickness of approx. $3 \mathrm{~mm}$ from the occipital lobe and the two gyri around the calcarine sulcus were blocked ( $\mathbf{b}, \mathbf{f}$, and $\mathbf{j})$. Blocked samples contained the V1/V2 border and parts of V2 in all cases. Samples show high transparency after labelling and clearing, especially in the ROls of the gyri crowns (c, g, and k; grid size: $1 \times 1 \mathrm{~mm}$ ). Overview acquisitions with the highest possible FOV and a resolution of approx. $10 \mu \mathrm{m}$ isotropic of the vessel label (d, $\mathbf{h}$, and I; scale bars: 3 $\mathrm{mm}$ respectively). Already at this resolution, the V1/V2 border is visible (see especially panel I). 

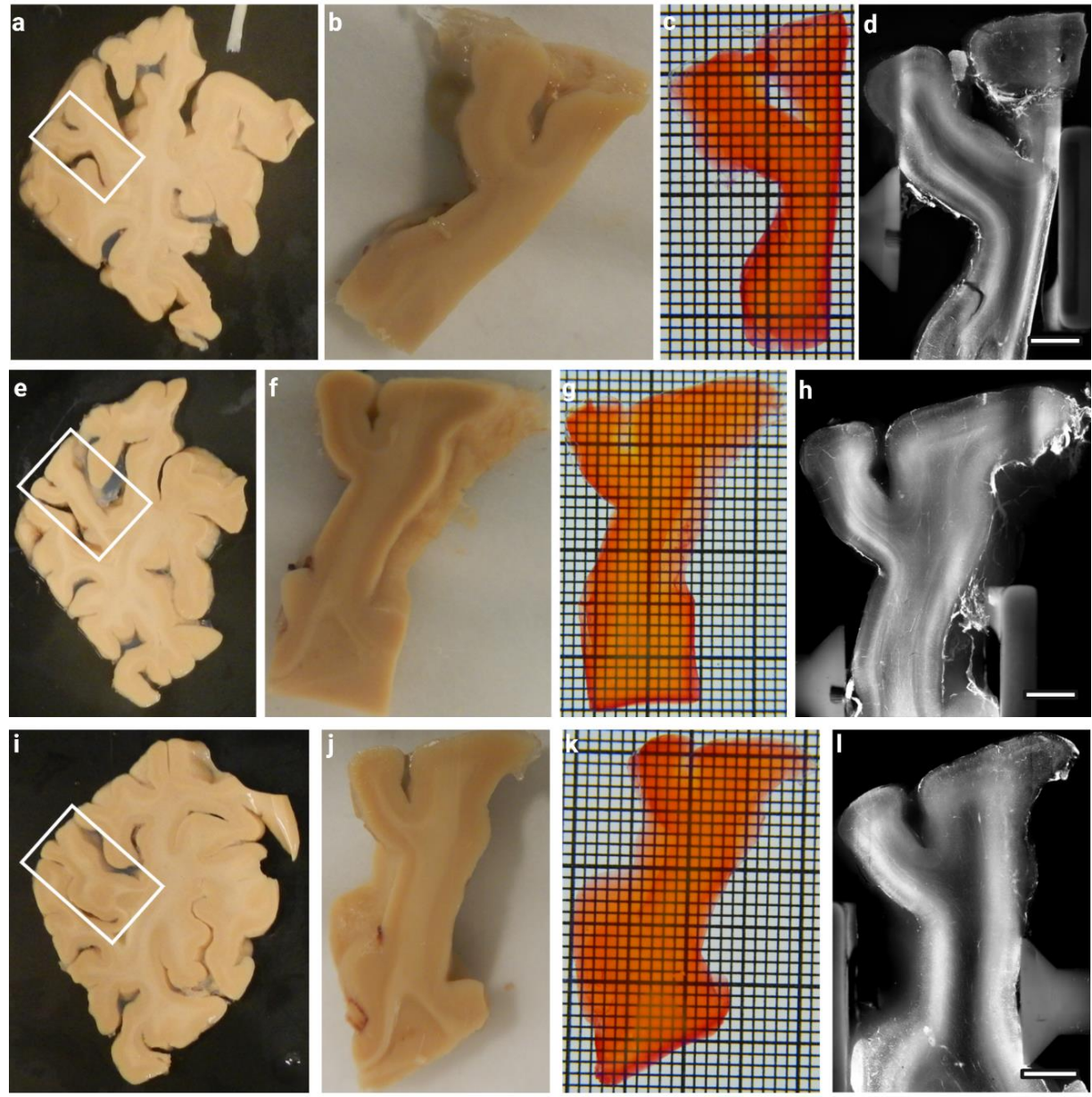

Supplementary Figure 2: Overview of Occipital lobe 2 samples. Three consecutive coronal slices (a, e, and I; direction posterior to anterior) where cut at a thickness of approx. $3 \mathrm{~mm}$ from the occipital lobe and samples containing a gyrus next to the calcarine sulcus were blocked (b, $\mathbf{f}$, and $\mathbf{j}$ ). Blocked samples contained the V1/V2 border and parts of V2 in all cases. Samples show high transparency after labelling and clearing, especially in the ROls of the gyri crowns (c, g, and k; grid size: $1 \times 1 \mathrm{~mm}$ ). Legend continued on the next page. 
Supplementary Figure 2: Continued. Overview acquisitions with the highest possible FOV and a resolution of approx. $10 \mu \mathrm{m}$ isotropic of the vessel label (d, h, and l; scale bars: $3 \mathrm{~mm}$ respectively). Already at this resolution, the V1/V2 border is visible (see especially panel $\mathbf{h}$ ).

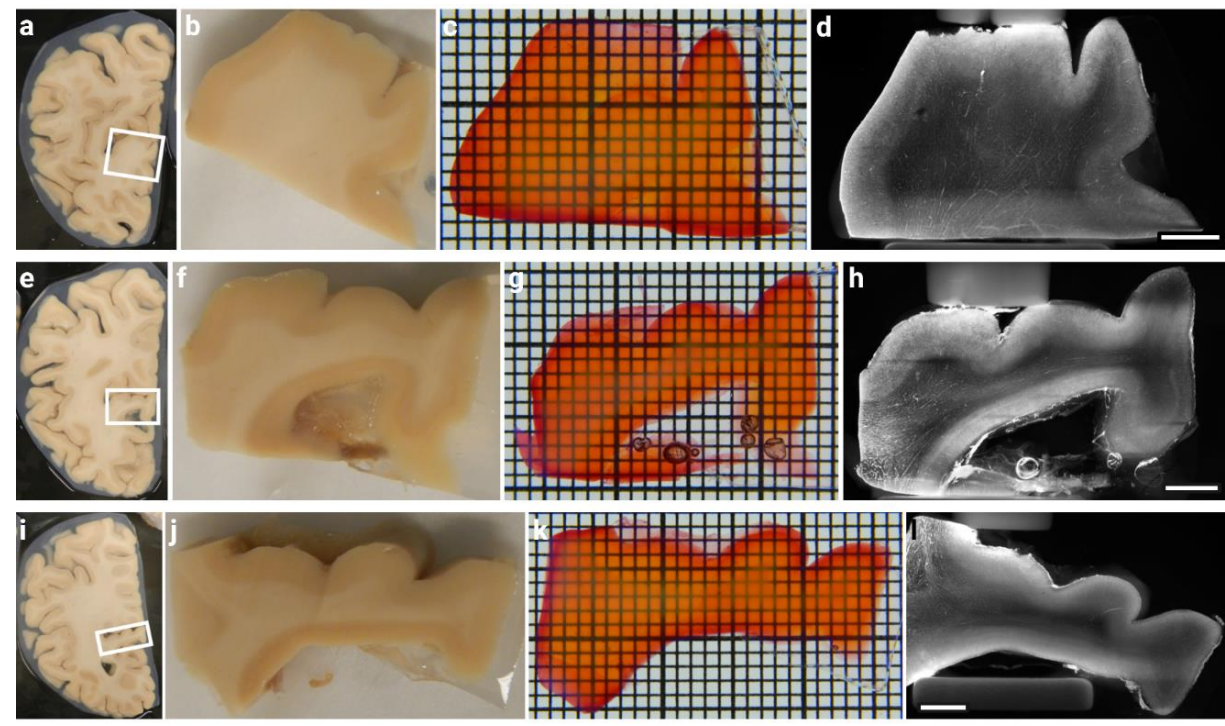

Supplementary Figure 3: Overview of Occipital lobe 3 samples. Three consecutive coronal slices (a, e, and I; direction posterior to anterior) where cut at a thickness of approx. $3 \mathrm{~mm}$ from the occipital lobe and samples containing a gyrus next to the calcarine sulcus were blocked (b, $\mathbf{f}$, and $\mathbf{j})$. Blocked samples contained the V1/V2 border and parts of V2 in all cases. Samples show high transparency after labelling and clearing, especially in the ROIs of the gyri corwns (c, g, and k; grid size: 1x1 mm). Overview acquisitions with the highest possible FOV and a resolution of approx. $10 \mu \mathrm{m}$ isotropic of the vessel label $(\mathbf{d}, \mathbf{h}$, and $\mathbf{l}$; scale bars: $3 \mathrm{~mm}$ respectively). Already at this resolution, the V1/V2 border is visible (see especially panel $h$ ). 


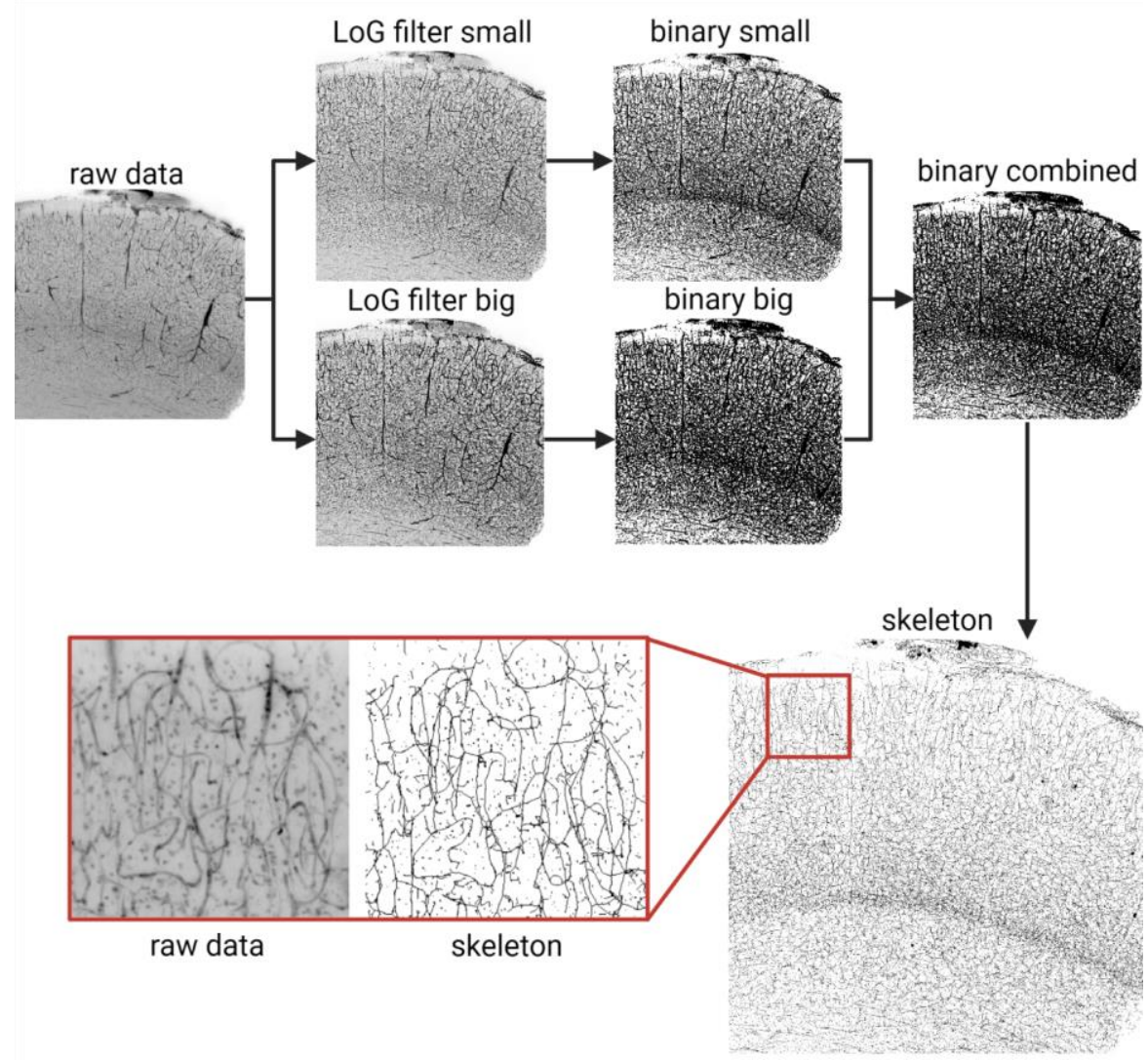

Supplementary Figure 4: Workflow for creating the vessel skeleton. Raw data was first filtered with a 3D Laplacian of Gaussian filter with a FIJI plugin. Two different filter settings were applied: Raw data was filtered with a small kernel size to enhance the small blood vessels. At this setting, the larger blood vessels appeared fragmented however, both in the filtered channel and the resulting skeleton. To represent the bigger blood vessels more accurately, a bigger kernel size was chosen, which resulted in large vessels appearing as continuous, filled structures, while maintaining the same width as compared to the raw data Legend continued on the next page. 
Supplementary Figure 4: Continued. Of these two filtered data sets, binary masks were created with FIJI and imported into Vision4D (arivis AG, Munich, Germany), where both masks were added into a combined binary channel. This channel was used as the source for a custom-made python script executed as a Vision4D plugin to generate the skeleton of the vessel channel. 
Chapter 3 - angioMASH 


\section{Taking MASH to the next level: A cost-}

\section{effective high-throughput pipeline for the investigation of cytoarchitecture in} large human brain slices
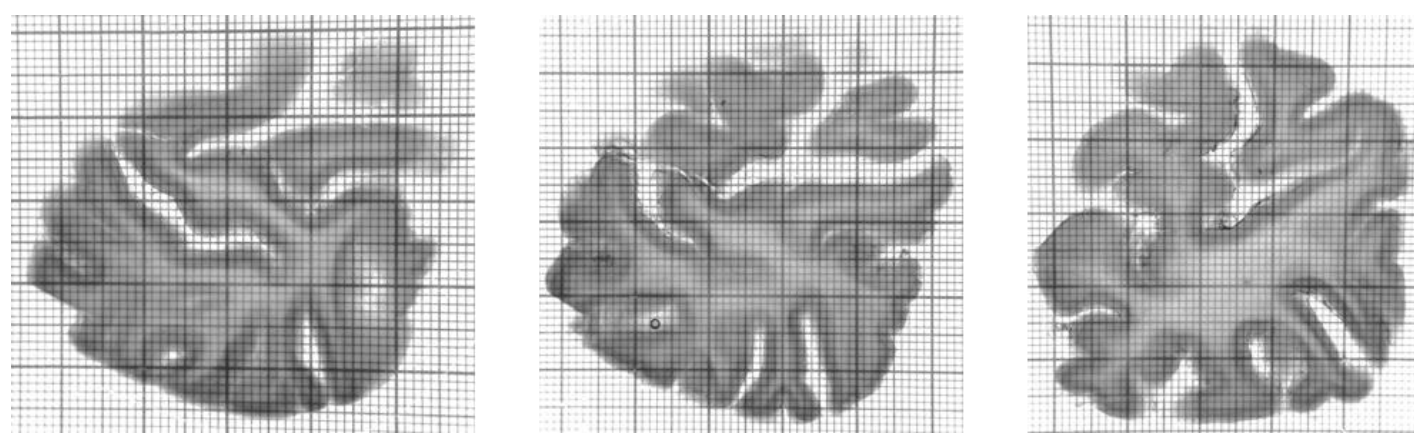

Consecutive coronal human occipital lobe slices cleared with MASH.

(in preparation)

Sven Hildebrand, Shubharthi Sengupta, Anna Schueth, Andreas Herrler, Alard Roebroeck 


\section{Abstract}

The performance of many optical clearing protocols has considerably improved in the last few years, such that now even notoriously difficult specimens such as highly myelinated human brain tissue can be rendered highly transparent. However, tissue clearing is still routinely performed on relatively small samples, especially in the case of the human brain, and compared to its size. Recent advances in histological tissue processing now allow scaling up the clearing process considerably towards much larger samples. Yet so far, these methods have considerable drawbacks in their feasibility towards routine implementation, especially in standard laboratories. Here, we present an updated version of our recently published MASH protocol, which allows for the clearing of very large human brain tissue samples (entire $3 \mathrm{~mm}$ thick coronal occipital lobe slices) and the labelling of cytoarchitecture therein. This new pipeline is cost-efficient and easy to implement, so that even standard labs can apply it realistically to routinely process very large samples. At the same time, the use of rapid prototyping using $3 \mathrm{D}$ printing to create custom clearing equipment is scalable enough to be adjusted to different clearing methods, sample sizes or tissue types. Our new pipeline therefore has the potential to advance optical clearing and labelling of large human tissue samples towards a more robust and routine implementation in the nascent field of 3D histology. 


\section{Introduction}

Despite the recent advances in histological and imaging methods, the investigation of micro- and mesoscopic anatomical features over large parts of the human brain is still a challenge. Imaging techniques need to have both the resolution to properly visualise these features at the microscopic level, but also have to cover large extents of tissue to view these features in their spatial context. Currently, the method of choice for investigating mesoscopic features, structures tens to hundreds of micrometre in size such as the layered cortical cytoarchitecture, is the 3D reconstruction of thousands of 2D brain sections. While this has yielded exceptional results ${ }^{1,2}$ and is expected to continue to do so in the future, optical clearing as a 3D histological method is providing a promising alternative for this type of investigation. Because optical clearing and 3D histology ultimately have the potential to yield faster and less labour-intensive processing pipelines, they are expected to become standard techniques in many histological laboratories.

Optical clearing is already applied with increasing frequency in the structural investigation of brain (and other) tissue. Until recently, cleared human brain samples were still relatively small in their lateral extent $(\sim 5-20 \mathrm{~mm})$ as emphasis was primarily on improving the clearing and labelling for thicker pieces of tissue ${ }^{3-9}$. The lateral size however, was so far limited mainly by the microscope systems as well. As new systems have been recently introduced to tackle this particular shortcoming ${ }^{10,11}$ (see also Chapter 5 of this thesis), efforts 
are being made by some groups to scale up the histological pipeline towards very large slices of human brain tissue, and even entire organs ${ }^{12,13}$. Even with the new imaging platforms however, the lightmicroscopy investigation of complete slices of more than a centimetre thickness, let alone entire cleared brains, is currently unrealistic and the processing of centimetre thick samples takes over 2 months ${ }^{13}$. The elasticising of human brain slices to allow for deep and fast label penetration seems to be a promising approach but so far, only the clearing has been demonstrated on $2 \mathrm{~mm}$ thick whole brain slices, not the labelling. This approach also relies on elaborate machinery to introduce the labels ${ }^{12}$.

A fast, cheap and easy-to-implement histological pipeline for 3D investigation of very large brain slices (several millimetres thick and many centimetres in lateral extend) is currently not available. This would be highly desirable however, as this would also enable standard histological laboratories to harness the full potential of tissue clearing for neuroanatomical studies. Here we present an updated version of our recently published MASH approach ${ }^{3}$, which is based upon an adjusted iDISCO $+{ }^{14}$ protocol, optimised for human brain tissue, and paired with several economic stainings to label cytoarchitecture. This new high-throughput version has been created with the aim of clearing and labelling entire coronal occipital lobe sections of several millimetres thickness. We use custom-made 3D printed clearing containers, compatible with the corrosive organic solvents used in the clearing technique, to accommodate multiple coronal slices in 
parallel. This pipeline can clear and stain large portions of human occipital lobes within 10 days. As shown in the original MASH protocol, the small molecule labels penetrate deep into the tissue using only passive diffusion, even in the very large samples processed in this study. In this first proof of concept, we demonstrate the high transparency and staining quality of these large samples, by acquiring mesoscopic overview scans with oblique light-sheet fluorescence microscopy. 


\section{Materials and Methods}

\section{Human brain tissue}

Brain tissue samples were taken from two different human body donors (Occipital lobe 1: 82 year old male; Occipital lobe 2: 101 year old female; no known neuropathological diseases respectively) of the body donation program of the Department of Anatomy and Embryology, Maastricht University. The tissue donors gave their informed and written consent to the donation of their body for teaching and research purposes as regulated by the Dutch law for the use of human remains for scientific research and education ("Wet op de Lijkbezorging"). Accordingly, a handwritten and signed codicil from the donor posed when still alive and well, is kept at the Department of Anatomy and Embryology Faculty of Health, Medicine and Life Sciences, Maastricht University, Maastricht, The Netherlands.

Brains were first fixed in situ by full body perfusion via the femoral artery. Under a pressure of 0.2 bar the body was perfused by 101 fixation fluid (1.8 vol \% formaldehyde, $20 \%$ ethanol, $8.4 \%$ glycerine in water) within 1.5-2 hours. Thereafter the body was preserved at least 4 weeks for post-fixation submersed in the same fluid. Subsequently, brains were recovered by calvarial dissection and stored in $4 \%$ paraformaldehyde in $0.1 \mathrm{M}$ phosphate buffered saline (PBS) for 14-30 months. 


\section{Preparation of human brain samples}

Each of the occipital lobes was cut into $3 \mathrm{~mm}$ thick coronal slices on a rotation slicer (ritterwerk $\mathrm{GmbH}$, Gröbenzell, Germany). To keep the brain tissue stabilized during slicing, the occipital lobes were embedded in a cylinder of $4 \%$ agar in $\mathrm{H}_{2} \mathrm{O}(\mathrm{w} / \mathrm{v})$. To achieve this, the tissue was placed in a plastic pipe with $12 \mathrm{~cm}$ diameter and the pipe was fixed with putty onto a plastic plate. Once filled with agar, the pipe was surrounded with crushed ice to accelerate solidification of the agar. The agar ring around the coronal slices was trimmed, but not completely removed unless it detached by itself as it serves to keep unattached gyri in place. Of each occipital lobe, the first 6 slices starting at the posterior pole ( 1.8 $\mathrm{cm}$ of the occipital lobe) were processed with the high-throughput version of MASH ${ }^{3}$.

\section{High-throughput clearing and labelling of human brain samples}

The complete procedure was carried out in custom-made 3D printed sample holder discs and tower (see next section). The tower was placed into a $110.6 \mathrm{~mm}$ inner diameter cylindrical polytetrafluorethylen (PTFE) container (Thermo Fisher Scientific, Waltham, Massachusetts, U.S.). For all steps, 11 of every solution described below was used, which was sufficient to cover all 6 samples of each lobe. For a fully loaded clearing tower, about twice that volume would be needed and twice the amount of tissue can be processed. The container was kept on a magnet stirrer at $350 \mathrm{rpm}$ throughout the incubations. Samples were dehydrated 1 h each in 20, 40, 60, 80, 
$100 \%$ methanol $(\mathrm{MeOH})$ at room temperature (RT) and $1 \mathrm{~h}$ in $100 \%$ $\mathrm{MeOH}$ at $4{ }^{\circ} \mathrm{C}$. Samples were then bleached overnight in freshly prepared, chilled $5 \% \mathrm{H}_{2} \mathrm{O}_{2}$ in $\mathrm{MeOH}(\mathrm{v} / \mathrm{v})$ at $4{ }^{\circ} \mathrm{C}$. The next day samples were rehydrated in $80,60,40,20 \% \mathrm{MeOH}$ at RT for $1 \mathrm{~h}$ respectively. This was followed by permeabilisation for $2 \mathrm{x} 1 \mathrm{~h}$ in $0.2 \%$ PBST (phosphate buffered saline $+0.2 \%(\mathrm{v} / \mathrm{v})$ Triton $\mathrm{x}-100$ adjusted to $\mathrm{pH}$ 7.4) a second bleaching step in $50 \%(\mathrm{w} / \mathrm{v})$ aqueous potassium disulfite solution for $1 \mathrm{~h}$ at RT. The solution was stirred at approx. $70^{\circ} \mathrm{C}$ until all precipitated crystals were dissolved and filtered right before use. This solution can be reused several times. Samples were thoroughly rinsed $5 \mathrm{x}$ in distilled water and washed for another hour in distilled water at RT. Then, samples were MASH-NR stained in $0.001 \%$ neutral red in McIlvain buffer $^{15}(\mathrm{w} / \mathrm{v})$ at $\mathrm{pH} 4$ for 5 days. Samples were flipped after half the incubation time. Samples were then washed again $2 \mathrm{x} 1 \mathrm{~h}$ in McIlvain at $\mathrm{pH}$ 4, dehydrated $1 \mathrm{~h}$ each in 20, 40, 60, 80, 2x $100 \%$ $\mathrm{MeOH} / \mathrm{H}_{2} \mathrm{O}(\mathrm{v} / \mathrm{v})$ and delipidated for $1 \mathrm{~h}$ each in $66 \% \mathrm{DCM} / 33 \%$ $\mathrm{MeOH}$ and 2x $100 \%$ DCM. Finally, samples were immersed in ethyl cinnamate (ECi) overnight for refractive index (RI) matching and ECi was exchanged the next morning. 

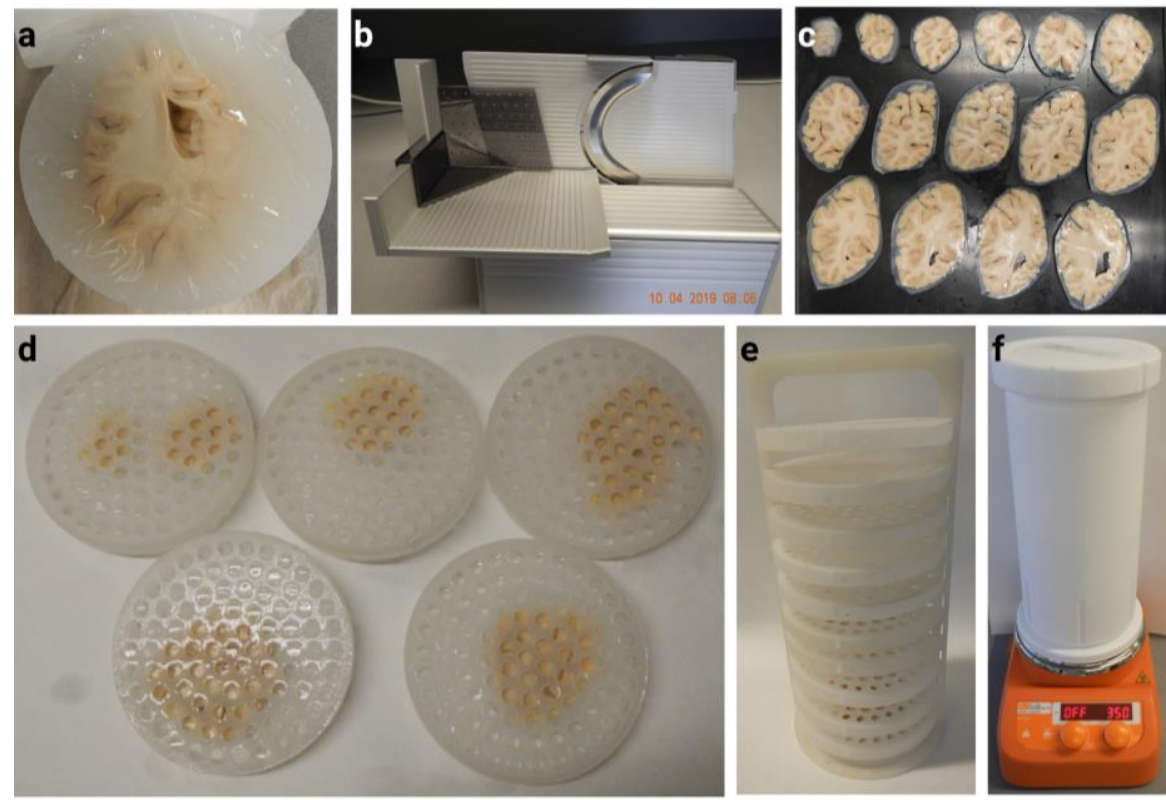

Figure 1: Overview of the high-throughput tissue-processing pipeline. Whole occipital lobes were embedded in $4 \%$ agar (a) and cut into $3 \mathrm{~mm}$ thick coronal slices on a commercial rotation slicer (b). The surrounding agar ring was trimmed with a scalpel (c) to better accommodate the samples in the sample holder discs (d). The SLS printed tower (e) can fit up to 10 sample holder discs with a $10 \mathrm{~cm}$ inner diameter and inner height of $5 \mathrm{~mm}$. This amounts to a theoretical maximal tissue volume of over $390 \mathrm{ml}$. The PTFE container (f) can accommodate a volume of approx. 2 I of staining and clearing chemicals with a fully loaded tower, guaranteeing a sufficient amount of staining and clearing solutions for the large tissue volume, while fitting on a compact magnet stirrer. 


\section{$3 D$ printed sample holders}

Designs for 3D printable sample holders were created in SOLIDWORKS (Dassault Systèmes SolidWorks Corporation, Waltham, US) and FreeCAD (version: 0.18; https://www.freecadweb.org).

SLS printed sample holders: A custom made sample holder optimized for coronal lobe slices of up to $5 \mathrm{~mm}$ thickness was produced from polypropylene (PP), after initial tests confirmed that PP is chemically resistant to DCM (although the material undergoes slight expansion during the DCM incubations and reverts to its original size after DCM has completely evaporated). The sample holder consists of a tower and perforated sample holder discs with lids that hold the individual samples. A first prototype (Fig. $1 \mathrm{~d}-\mathrm{f}$ and Suppl. Fig. 1) was produced via Selective Laser Sintering (SLS) by Materialise NV (Leuven, Belgium).

FDM/FFF printed sample holders: Following initial tests, an adjusted design was developed to provide more stability for the sample holder discs within tower, while adjusting the design to be printable with more affordable Fused Deposition Molding (FDM)/ Fused Filament Fabrication (FFF) printers at the same time. These modified versions were printed on an Ender 3 Pro printer (Creality, Shenzhen, China). Fiberology PP filament (Fiberlab S.A., Brzezie, Poland) was printed onto a PP plate coated with Magigoo PP adhesive (Thought3D Ltd, Malta). The filament was printed at $230{ }^{\circ} \mathrm{C}$ nozzle temperature and $100{ }^{\circ} \mathrm{C}$ bed temperature with a 0.4 brass nozzle at $0.1 \mathrm{~mm}$ layer height 
and $0.35 \mathrm{~mm} / \mathrm{s}$. Filling density of the structure was set to $50 \%$ and support structures where printed as a grid with $25 \%$ filling to ensure good quality of the print. Additionally, to further lend support during the printing process and avoid the tower poles from swinging, a support connection was introduced at about half the tower height (see red arrow Fig. 2b), which was removed using wire cutters and scalpel, together with the support structure, after printing.

\section{Light-sheet fluorescence microscopy}

The samples were imaged on the ct-dSPIM (cleared tissue dual-view Selective Plane Illumination Microscopy; Applied Scientific Instrumentation, Eugene, US) prototype set-up (see Chapter 5). The set-up was equipped with multi-immersion detection objectives (Applied Scientific Instrumentation, Eugene, US/Special Optics, Denville, US), suitable for a RI range from 1.33 to 1.56 and with a working distance (WD) of $12 \mathrm{~mm}$. Both, the numerical aperture (NA) and effective focal length (EFL) vary with RI, but for ECi the NA is $\sim 0.43$ and EFL $\sim 11.2 \mathrm{~mm}$, with a magnification factor at $552 \mathrm{~nm}$ of 17.9x. For excitation, an OBIS LS $552 \mathrm{~nm}$ 40mW laser line (Coherent Inc., Santa Clara, US) was used. Samples were imaged with $10 \mathrm{~ms}$ exposure time at the lowest laser intensity setting with 4x4 binning (512x512 pixels, amounting to $1.45 \mu \mathrm{m}$ binned pixel size) and a step size of $23.2 \mu \mathrm{m}$, in order to acquire a fast, mesoscopic overview of the large samples (see Chapter 5). 


\section{Data processing}

The datasets were further downsampled 16x in plane (32x32 pixels), to match the step size of the microscope and produce an isotropic dataset of $16.4 \mu \mathrm{m}$ isotropic after deskewing. Downsampling was done in FIJI $^{16}$ and both deskewing and stitching of the datasets were performed in the BigStitcher plugin ${ }^{17}$ for FIJI. After export of the fused datasets, the volume was resliced in FIJI to show the coronal plane of the tissue slice (YZ plane of the image volume). For the creation of all figures, BioRender was used (https://www.biorender.com). 


\section{Results}

Our new high-throughput version of the MASH protocol allows for rapid optical clearing and cytoarchitecture labelling in very large tissue volumes (Fig. 1). Large parts of human occipital lobes can be histologically processed and readied for microscopic imaging within 10 days. In the SLS printed prototype (Fig $1 \mathrm{~d}$ and e; Suppl. Fig.1), a maximum tissue volume of approx. $390 \mathrm{ml}$ (in slices of a diameter of $10 \mathrm{~cm}$ ) can be processed in a single round of clearing. This pipeline massively extends the current clearing and labelling capacities beyond the original MAH protocol, which could already process $\sim 5 \times 20 \times 30 \mathrm{~mm}$ large samples. At the same time, the high-throughput pipeline requires mostly standard lab equipment and little additional or costly hardware.

To further reduce the cost of the pipeline (see Suppl. Table 1), we replaced the most expensive hardware item, the SLS printed tower and sample holder discs. The new design can be reliably printed with inexpensive PP filament on standard FDM/FFF desktop printers (Fig. 2), making the whole set-up for the histological pipeline even more economical. The FDM/FFF prints have a worse resolution, but the printing quality is sufficient for all the parts at this large scale. The slightly smaller filament printed tower fits 6 sample holder discs corresponding to a maximum tissue volume of $235 \mathrm{ml}$. 

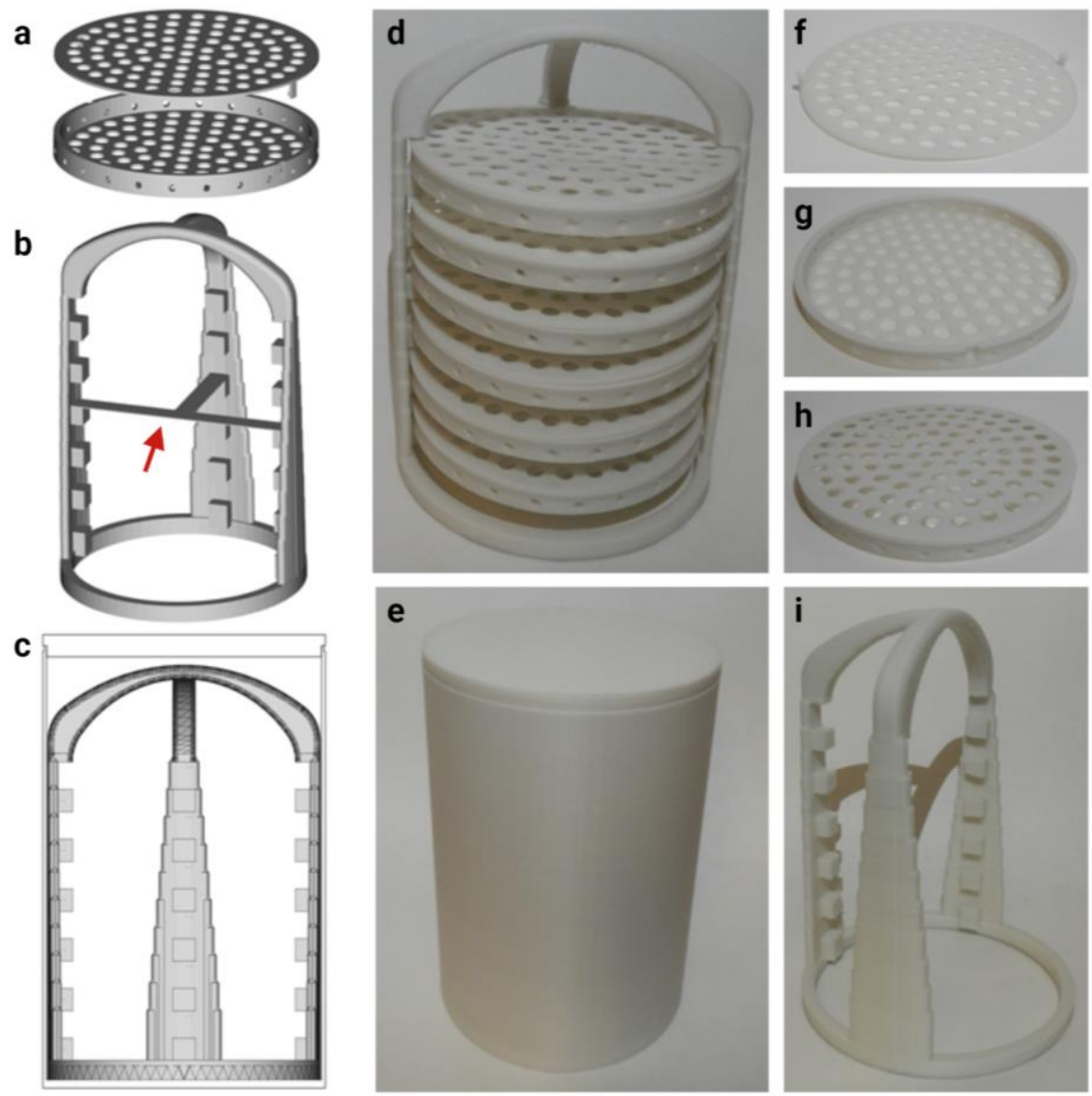

Figure 2: Modified clearing tower version for FDM/FFF printing. (a) The dovetails on the lids and corresponding groves on the sample holder discs were modified to remove $90^{\circ}$ angles for better printability. (b) To prevent the tower poles from swinging through the movement of the nozzle during printing, additional support elements (red arrow) were introduced and the tower pole width was increased as well. This together with the third pole added to the design also gives more support to the sample holder discs. (c) Tower inside the 3D printed clearing container. (d - i) Final prototype printed with PP filament. 
To provide a proof of principle, we processed the posterior portions of two human occipital lobes (Fig. 3 and Suppl. Fig. 2-13). Six consecutive $3 \mathrm{~mm}$ thick slices starting at the occipital pole were cleared and labelled in the high-throughput MASH pipeline. Even the dense, central white matter in these large samples became highly transparent (Fig. 3). Within each batch, the labelling appeared homogeneous and was independent of the sample size. The differences in colouration between both occipital lobe batches likely arose through a slightly higher concentration of the staining solution, as even a very small amount of additional dye powder can visibly increase the colour of the neutral red solution.

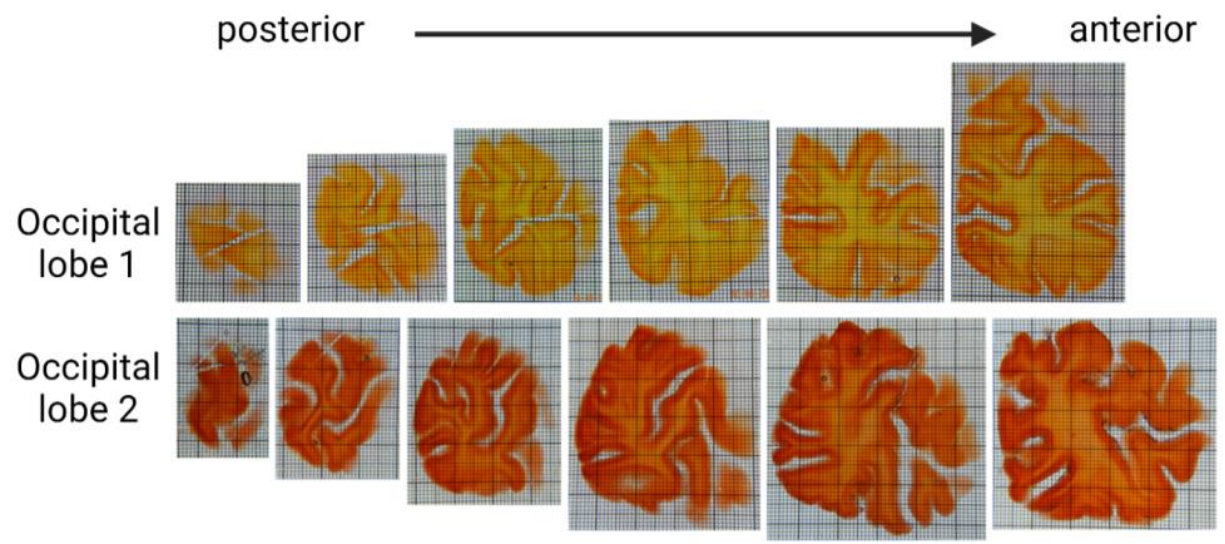

Figure 3: High-throughput clearing of two human occipital lobes. All processed coronal slices of occipital lobe 1 (top) and occipital lobe 2 (bottom) arranged with the posterior pole towards the left side of the image and the most anterior sample towards the right. All panels are adjusted to the same scale (grid size: 1x1 mm smallest squares; $10 \times 10 \mathrm{~mm}$ boldly lined squares).

In order to assess the labelling quality of the samples, mesoscopic 
light-sheet overview scans of the largest slice of occipital lobe 1 and 2 were acquired (Fig. 4). At mesoscopic resolution of $16.4 \mu \mathrm{m}$ isotropic, anatomical landmarks such as the tentative V1/V2 border are already visible (Fig. 4 red arrows), as are several cytoarchitectonic layers. Particularly layer I and the grey matter/white matter border are well visible. Within the cortical sheet, the infragranular layers $\mathrm{V}$ and VI can be distinguished and in V2, a particularly densely labelled layer in the middle of the cortex is presumed to represent layer IV. In V1, layer IVb can be faintly distinguished as a slightly paler stripe between the more densely labelled layers IVa and IVc, although this is challenging at this mesoscopic resolution. As expected, the small molecule MASH-NR label penetrates the entire depth of the large samples after 5 days of passive incubation (see Suppl. Fig. 14 and 15; Suppl. Video 1 and 2). 
a

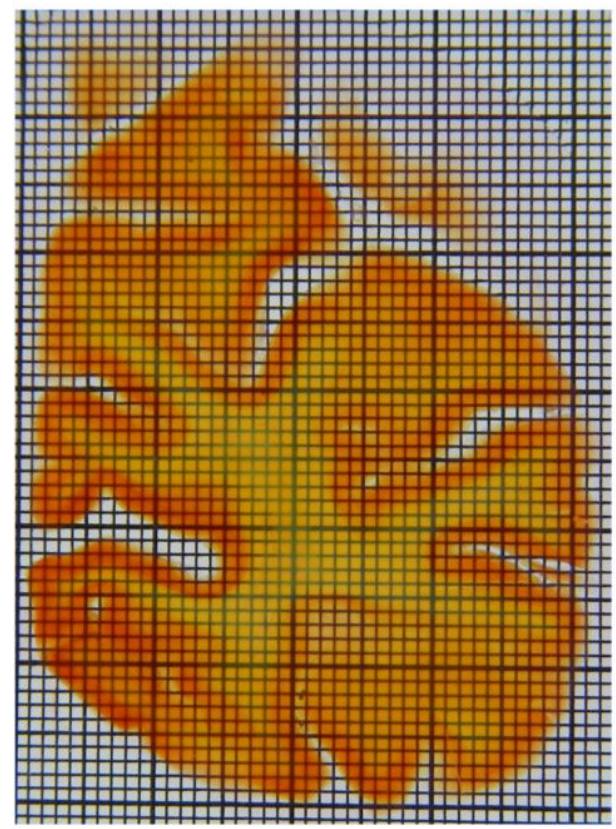

Occipital lobe 1

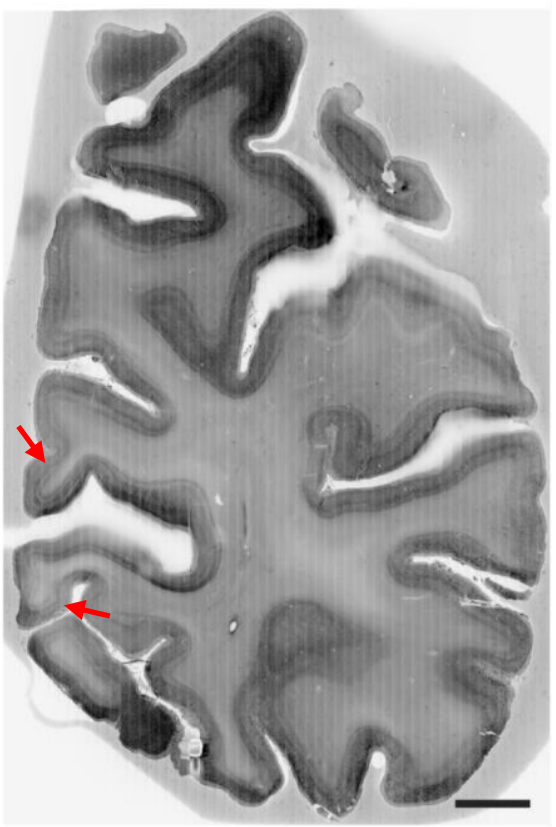

b

Occipital lobe 2
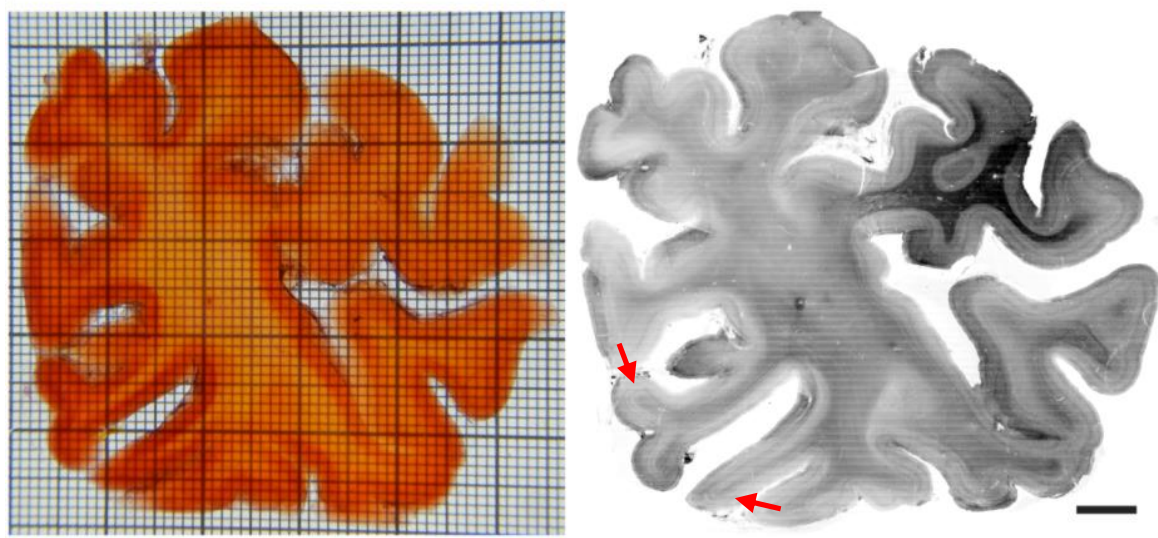

Figure 4: Mesoscopic overview acquisitions of anterior most occipital lobe

slices. Comparison between the macroscopic images of the cleared samples (right side) and the stitched, resliced image volumes in inverted greyscale (left side) of the two largest slices of occipital lobe 1 (a) and occipital lobe 2 (b). Legend continued on the next page. 
Figure 4: Continued. Both panels of the microscopy volumes show MIPs of approx. $50 \mu \mathrm{m}$ showing the largest extent of the samples. Anatomical landmarks such as the V1/V2 border can be distinguished even at mesoscopic resolution (arrows). Scale bars: $5 \mathrm{~mm}$ respectively; grid size: $1 \times 1 \mathrm{~mm}$ smallest squares; $10 \times 10 \mathrm{~mm}$ boldly lined squares. 


\section{Discussion}

We demonstrate an updated high-throughput version of MASH for the cost-efficient (see Suppl. Table 1), robust, and routine application of tissue clearing in multiple very large human brain samples in parallel for cytoarchitectonic investigations. The current set-up can process considerable parts of the human brain within a 10 day time span. As the tower and sample holder discs are 3D printed and therefore scalable, it would be feasible in the future to adjust the set-up, in order to accommodate entire sections of human or other large mammalian brains.

\section{Production of 3D printed sample holders}

Although PP is normally not biodegradable ${ }^{18}$, it is a recyclable polymer and its outstanding chemical properties make it ideal for the purpose of custom made tissue clearing hardware ${ }^{19}$. PP was therefore an obvious choice for the first prototype produced with SLS. This process allows for printing without support structures and therefore gives more freedom with regard to the shape of the print. The prints produced by this method were generally more rigid and the printing resolution was higher, so that the fitting of the components was generally more precise. This higher printing quality is offset however by a much higher cost of both the printer itself, and the higher production cost per item (especially when obtained from external companies rather than produced on site). For large-scale prints such as these, the quality provided by FDM/FFF printing is sufficient for its 
intended use and comes at much lower cost. FDM printing on site massively reduces the cost by two orders of magnitude as compared to the commercial SLS prints. Given that the printers themselves are cheaper to the same degree, this mode of production seems to be the most obvious choice, especially for smaller laboratories. Once the price for SLS printers is further reduced, this manufacturing process could replace filament printing, given its other advantages. One problem with the FDM/FFF prints is the waste generated by the support structures. Future design improvements could reduce this by e.g. printing the tower in parts without supports and assembling it later with an organic-solvent resistant adhesive. In general, the great flexibility of 3D printing enables rapid prototyping and adjustments of the size of the containers. Fields such as comparative neuroanatomy, which have to deal with unusual sample sizes ${ }^{20,21}$, might profit from this technology.

\section{High-throughput pipeline for human brain clearing}

One problem when slicing brain tissue is the preservation of its shape, including gyri that are not attached to the main part of the section. Although cutting much thicker slices as compared to traditional histology of several millimetres thickness helps alleviate this problem, it cannot be fully avoided by this measure alone. In order to give support to the tissue during the sectioning, we opted for embedding the samples in $4 \%$ agar. Even though the agar becomes very transparent after clearing, it is still visible in the microscopic images 
(see Fig. 4a) and can produce light scattering. Depending on the lightsheet microscope geometry, it can therefore be desirable to remove as much agar as possible. Manual removal of the agar is difficult and prone to damaging the cortex. A possible solution for that in the future could be the use of low-melting point alternatives to agar, such as, low-melting agarose $\mathrm{e}^{22}$ or gelatine as a cheaper alternative. In these cases, the embedding material could be removed by heating up the sample with warm water. Of course, this would necessitate fixing the tissue in place by some manner at this point. Tissue samples of the size introduced in this chapter necessitate an oblique light-sheet microscope geometry ${ }^{10,11}$ (see Chapter 5 of this thesis), as in conventional set-ups the light cannot penetrate the full lateral extent of the sample. Since the agar is located around the tissue slices, its impact on imaging quality is less severe. Whether the embedding material needs to be removed will depend on many factors such as sample thickness, transparency, wavelength of the fluorophore, and tissue type and should therefore be considered on a case-by-case basis.

\section{Outlook}

Although the data presented here is only intended to serve as a proof of concept, it becomes obvious that the tissue clearing, and in some cases even the labelling, are no longer the limiting factors in the rapidly evolving field of 3D histology. As shown here, hundreds of $\mathrm{cm} 3$ of brain tissue can be processed simultaneously and in short time. The 
next steps needed are the development of imaging systems and quantitative data analysis routines, which can accommodate such large samples and process them in a reasonable amount of time to avoid simply relocating the bottleneck of the entire pipeline elsewhere. Already the first light-sheet microscope systems have been introduced, capable of handling these large samples ${ }^{10,11,23}$ and further developments in this area are expected. One light-sheet microscope set-up and imaging method to address this problem is presented in Chapter 5 of this thesis. Simultaneously, many image visualisation and analysis companies have turned their attention to this particular issue and several freely available tools have been developed to address the analysis and visualisation of data of this large scope. Recently solutions have been introduced for multi-view fusion $^{24,25}$, stitching ${ }^{17,26}$, visualisation ${ }^{25,27}$, and compression ${ }^{24,28}$ for large light-sheet datasets. Although the ImageJ environment ${ }^{16}$ combines some of these tools, there is no open-source tool available for every processing step. Therefore, user-friendly tools for data handling seem to have the most pressing need for future improvements.

Despite these current shortcomings, the cost-effective highthroughput MASH pipeline presented here improves upon the first steps, which any 3D histology pipeline for very large human brain (and potentially other) tissue would need to address. This pipeline therefore opens the door towards the routine investigation of post mortem human brain tissue in health and disease at a truly unprecedented scale. 


\section{References}

1 Amunts, K. et al. BigBrain: An Ultrahigh-Resolution 3D Human Brain Model. Science 340, 1472, doi:10.1126/science.1235381 (2013).

2 Amunts, K., Mohlberg, H., Bludau, S. \& Zilles, K. Julich-Brain: A 3D probabilistic atlas of the human brain's cytoarchitecture. Science 369,988 , doi:10.1126/science.abb4588 (2020).

3 Hildebrand, S., Schueth, A., Herrler, A., Galuske, R. \& Roebroeck, A. Scalable Labeling for Cytoarchitectonic Characterisation of Large Optically Cleared Human Neocortex Samples. Scientific reports 9, 10880, doi:10.1038/s41598-019-47336-9 (2019).

$4 \quad$ Hildebrand, S. et al. hFRUIT: An optimized agent for optical clearing of Dil-stained adult human brain tissue. Scientific reports 10, 9950, doi:10.1038/s41598-020-66999-3 (2020).

5 Lai, H. M. et al. Next generation histology methods for three-dimensional imaging of fresh and archival human brain tissues. Nature communications 9, 1066, doi:10.1038/s41467-018-03359-w (2018).

6 Liebmann, T. et al. Three-Dimensional Study of Alzheimer's Disease Hallmarks Using the iDISCO Clearing Method. Cell reports 16, 1138-1152, doi:10.1016/j.celrep.2016.06.060 (2016).

7 Liu, A. K. et al. Bringing CLARITY to the human brain: visualisation of Lewy pathology in three dimensions. Neuropathology and applied neurobiology 42, 573-587, doi:10.1111/nan.12293 (2016).

8 Liu, A. K. L., Lai, H. M., Chang, R. C. C. \& Gentleman, S. M. Free of acrylamide sodium dodecyl sulphate (SDS)-based tissue clearing (FASTClear): a novel protocol of tissue clearing for three-dimensional visualisation of human brain tissues. Neuropathology and applied neurobiology 43, 346-351, doi:https://doi.org/10.1111/nan.12361 (2017).

9 Morawski, M. et al. Developing 3D microscopy with CLARITY on human brain tissue: Towards a tool for informing and validating MRI-based histology. Neuroimage

182 , 417-428, doi:10.1016/j.neuroimage.2017.11.060 (2018). 
10 Glaser, A. K. et al. Light-sheet microscopy for slide-free non-destructive pathology of large clinical specimens. Nature biomedical engineering 1, doi:10.1038/s41551-017-0084 (2017).

11 Glaser, A. K. et al. Multi-immersion open-top light-sheet microscope for high-throughput imaging of cleared tissues. Nature communications 10 , 2781, doi:10.1038/s41467-019-10534-0 (2019).

$12 \mathrm{Ku}, \mathrm{T}$. et al. Elasticizing tissues for reversible shape transformation and accelerated molecular labeling. Nature methods 17, 609-613, doi:10.1038/s41592-020-0823-y (2020).

13 Zhao, S. et al. Cellular and Molecular Probing of Intact Human Organs. Cell 180, 796-812 e719, doi:10.1016/j.cell.2020.01.030 (2020).

14 Renier, N. et al. Mapping of Brain Activity by Automated Volume Analysis of Immediate Early Genes. Cell 165, 1789-1802, doi:10.1016/j.cell.2016.05.007 (2016).

15 Mcllvaine, T. C. A buffer solution for colourimetric comparison. Journal of Biological Chemistry 49, 183-186 (1921).

16 Schindelin, J. et al. Fiji: an open-source platform for biological-image analysis. Nature methods 9, 676-682, doi:10.1038/nmeth.2019 (2012).

17 Hörl, D. et al. BigStitcher: reconstructing high-resolution image datasets of cleared and expanded samples. Nature methods 16, 870-874, doi:10.1038/s41592-019-0501-0 (2019).

18 Arutchelvi, J. et al. Biodegradation of polyethylene and polypropylene. (2008).

19 Maddah, H. A. Polypropylene as a promising plastic: A review. Am. J. Polym. Sci 6, 1-11 (2016).

20 Herculano-Houzel, S. et al. The elephant brain in numbers. Frontiers in neuroanatomy 8, 46 (2014).

21 Marino, L. et al. Neuroanatomy of the common dolphin (Delphinus delphis) as revealed by magnetic resonance imaging (MRI). The Anatomical Record 268, 411-429, doi:https://doi.org/10.1002/ar.10181 (2002). 
22 Iulianella, A. Cutting thick sections using a vibratome. Cold Spring Harbor Protocols 2017, pdb-prot094011 (2017).

23 Sabdyusheva Litschauer, I. et al. 3D histopathology of human tumours by fast clearing and ultramicroscopy. Scientific reports 10, 17619, doi:10.1038/s41598-020-71737-w (2020).

24 Amat, F. et al. Efficient processing and analysis of large-scale light-sheet microscopy data. Nature protocols 10, 1679-1696, doi:10.1038/nprot.2015.111 (2015).

25 Pietzsch, T., Saalfeld, S., Preibisch, S. \& Tomancak, P. BigDataViewer: visualisation and processing for large image data sets. Nature methods 12 , 481-483, doi:10.1038/nmeth.3392 (2015).

26 Bria, A. \& lannello, G. TeraStitcher - A tool for fast automatic 3D-stitching of teravoxel-sized microscopy images. BMC Bioinformatics 13, 316, doi:10.1186/1471-2105-13-316 (2012).

27 Royer, L. A. et al. ClearVolume: open-source live 3D visualisation for lightsheet microscopy. Nature methods 12, 480-481, doi:10.1038/nmeth.3372 (2015).

28 Balázs, B., Deschamps, J., Albert, M., Ries, J. \& Hufnagel, L. A real-time compression library for microscopy images. bioRxiv, 164624, doi:10.1101/164624 (2017). 


\section{Appendix Chapter 4}

Supplementary Table 1. Estimates of the costs for the high-throughput MASH set-up and chemicals. Chemical costs are given as the sum for the processing of one entire batch of 6 occipital lobe slices with 1 I solution for each step as mentioned in the method section.

\begin{tabular}{rrr} 
Name & Function & Price [€] \\
\hline Rotation slicer & Hardware & 169.99 \\
FDM/FFF printed clearing container, tower, & & \\
sample holder set & Hardware & 20 \\
Methanol & Dehydration & 82.23 \\
Dichloromethane & Delipidation & 40.48 \\
Potassium disulphite & Bleaching & 14.25 \\
Hydrogen peroxide & Bleaching & 3.39 \\
Ethyl cinnamate & RI-matching & 78 \\
Neutral red solution & Staining & 0.06 \\
& & 408.4
\end{tabular}

Supplementary Video 1. Plane-by-plane view of the resliced MFS 16 scan of occipital lobe 1 slice 6. The sample is viewed along the YZ plane, to visualise its full extend. The volume is represented in inverted greyscale.

Supplementary Video 2. Plane-by-plane view of the resliced MFS 16 scan of occipital lobe 2 slice 6 . The sample is viewed along the $Y Z$ plane, to visualise its full extend. The volume is represented in inverted greyscale. 

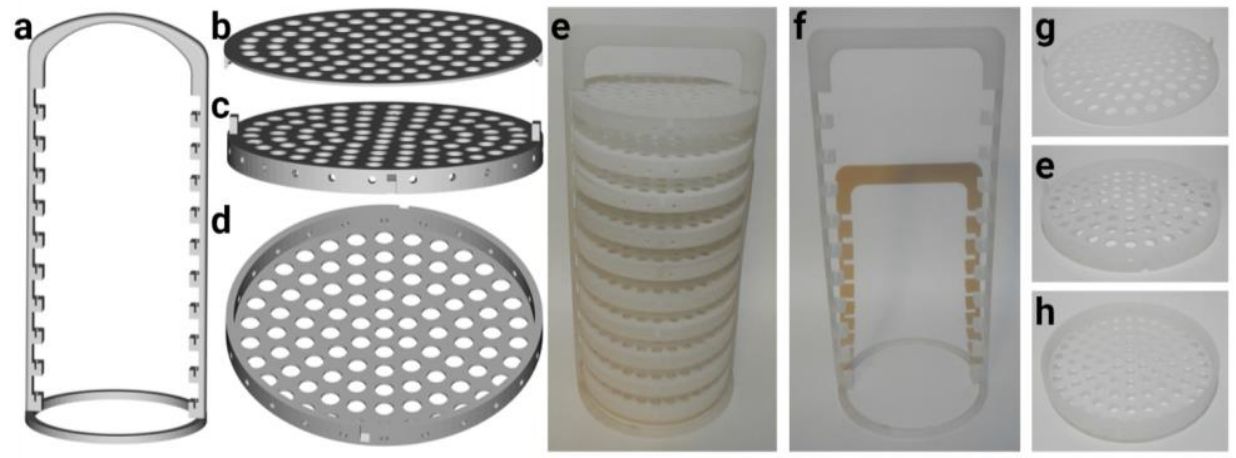

Supplementary Figure 1: Clearing tower and sample holder prototypes for SLS printing. (a) The tower can fit up to 10 sample holder discs at once. In order to provide support to the sample holders $(\mathbf{b}-\mathbf{d})$, grooves have been introduced into which the feet of the sample holders can be placed (see topside in $\mathbf{c})$. (e $-\mathbf{h}$ ) Final prototype of commercial SLS printing. 

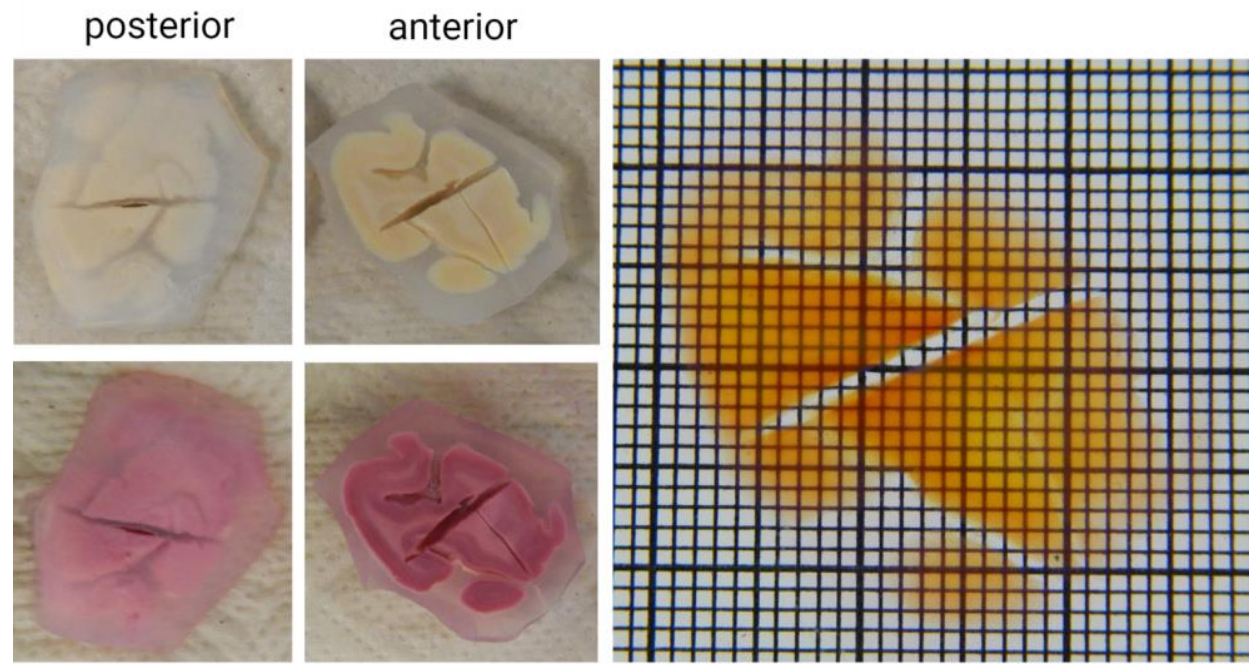

Supplementary Figure 2: Occipital lobe 1, slice 1 (posterior to anterior). Posterior views of the sample after bleaching (top) and staining (bottom) are shown in the left, anterior views on in the middle. The cleared and RI-matched sample is shown on the right (Grid: 1×1 mm smallest squares; $10 \times 10 \mathrm{~mm}$ bold squares). 

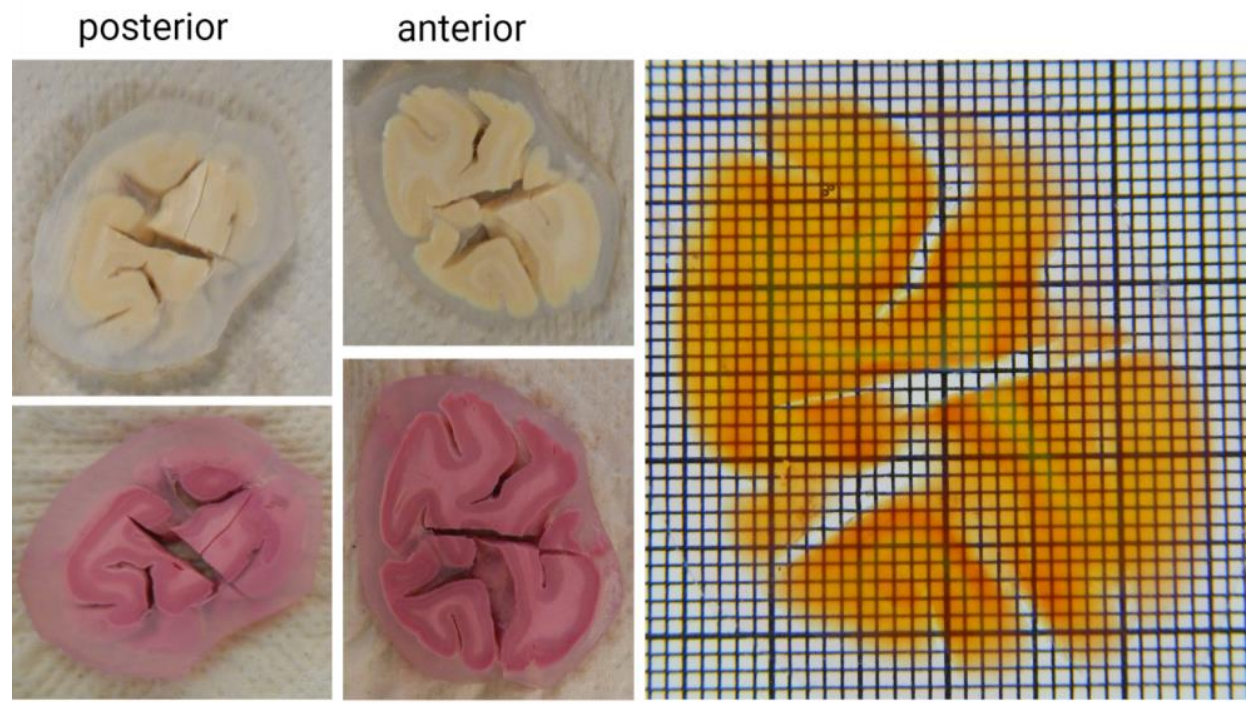

Supplementary Figure 3: Occipital lobe 1, slice 2 (posterior to anterior). Posterior views of the sample after bleaching (top) and staining (bottom) are shown in the left, anterior views on in the middle. The cleared and RI-matched sample is shown on the right (Grid: 1×1 mm smallest squares; $10 \times 10 \mathrm{~mm}$ bold squares). 


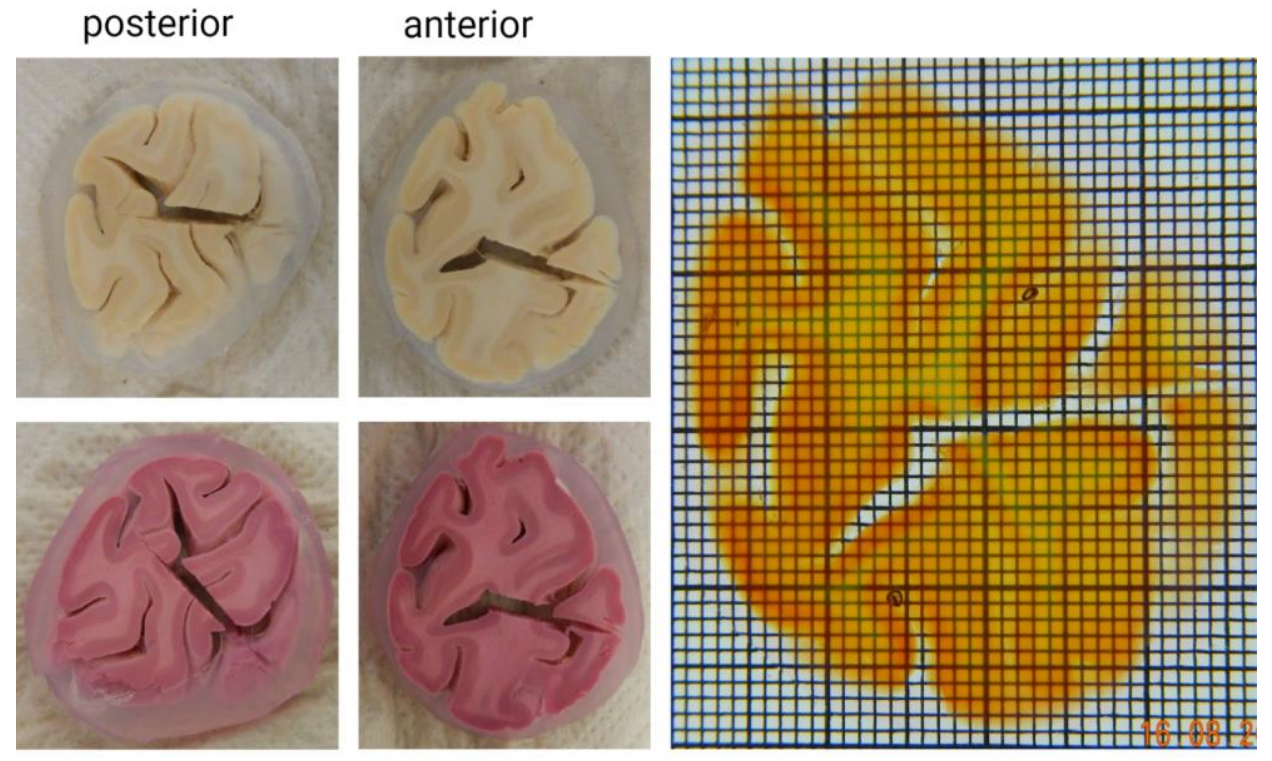

Supplementary Figure 4: Occipital lobe 1, slice 3 (posterior to anterior). Posterior views of the sample after bleaching (top) and staining (bottom) are shown in the left, anterior views on in the middle. The cleared and RI-matched sample is shown on the right (Grid: 1x1 mm smallest squares; $10 \times 10 \mathrm{~mm}$ bold squares). 


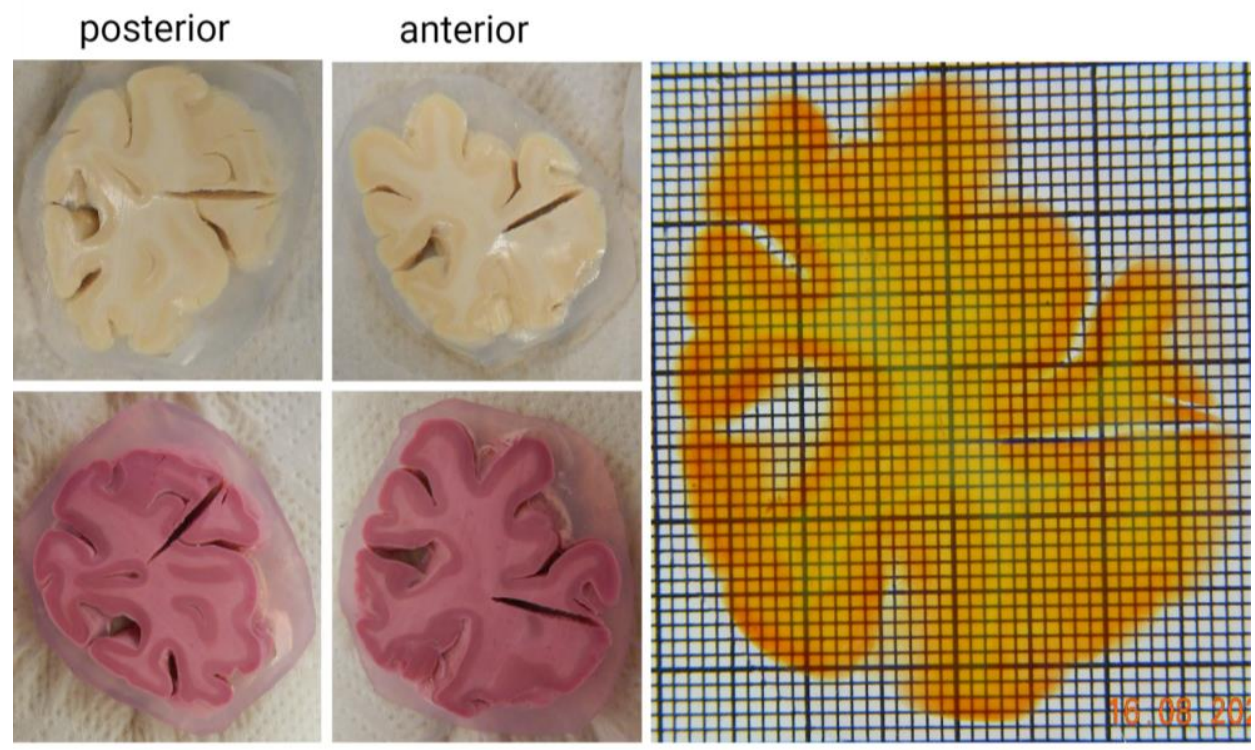

Supplementary Figure 5: Occipital lobe 1, slice 4 (posterior to anterior). Posterior views of the sample after bleaching (top) and staining (bottom) are shown in the left, anterior views on in the middle. The cleared and RI-matched sample is shown on the right (Grid: 1×1 mm smallest squares; 10x10 mm bold squares). 

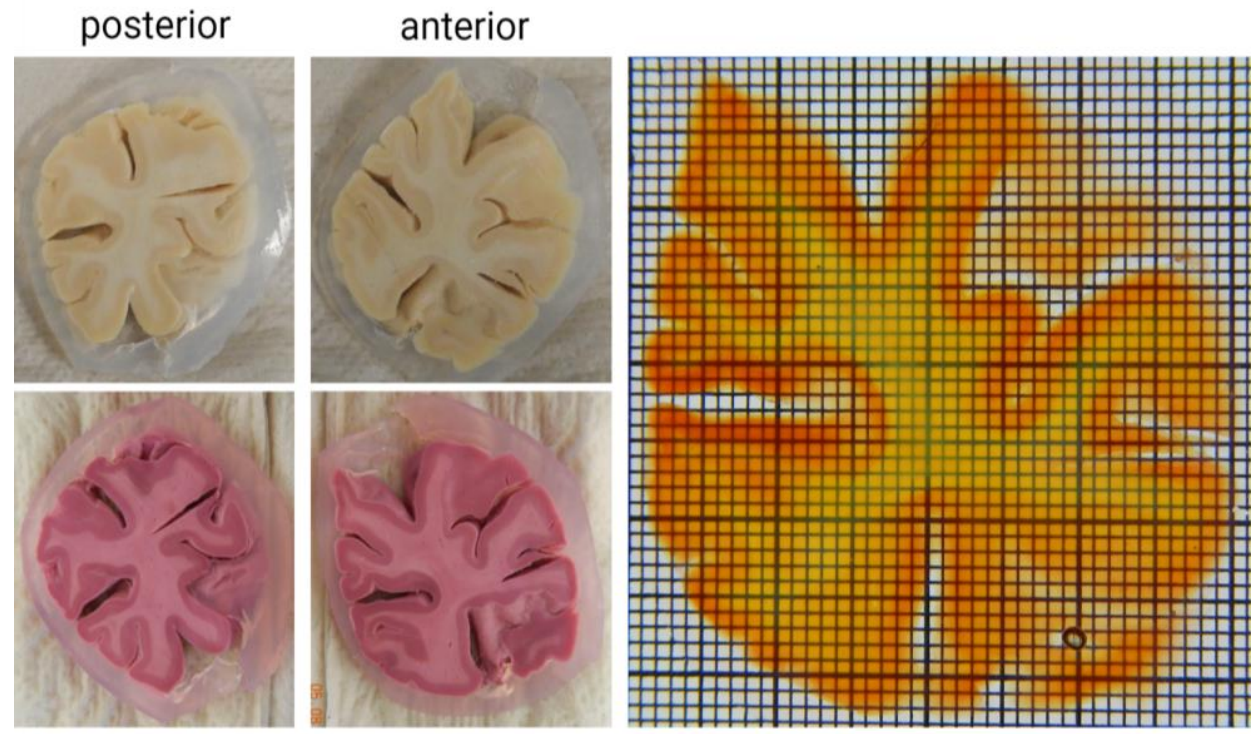

Supplementary Figure 6: Occipital lobe 1, slice 5 (posterior to anterior). Posterior views of the sample after bleaching (top) and staining (bottom) are shown in the left, anterior views on in the middle. The cleared and RI-matched sample is shown on the right (Grid: 1×1 mm smallest squares; $10 \times 10 \mathrm{~mm}$ bold squares). 


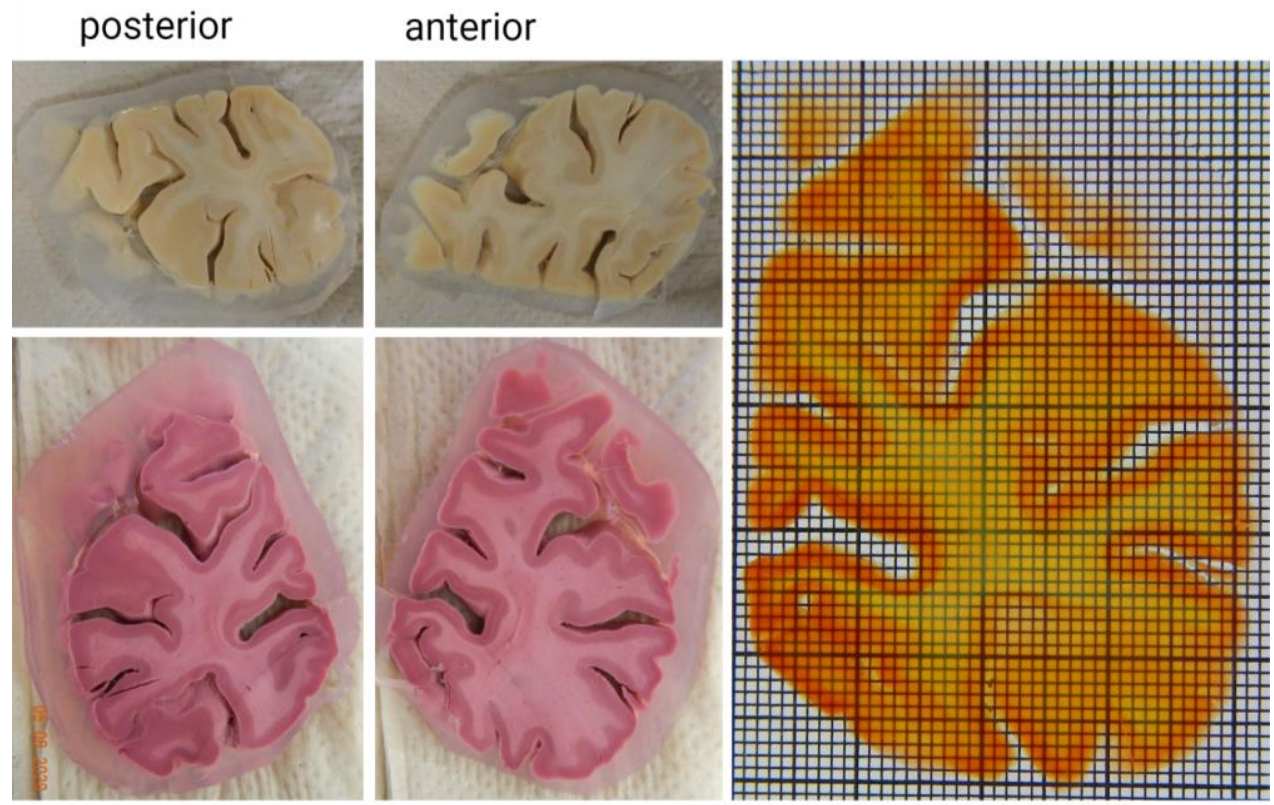

Supplementary Figure 7: Occipital lobe 1, slice 6 (posterior to anterior). Posterior views of the sample after bleaching (top) and staining (bottom) are shown in the left, anterior views on in the middle. The cleared and RI-matched sample is shown on the right (Grid: 1×1 mm smallest squares; 10×10 mm bold squares). 


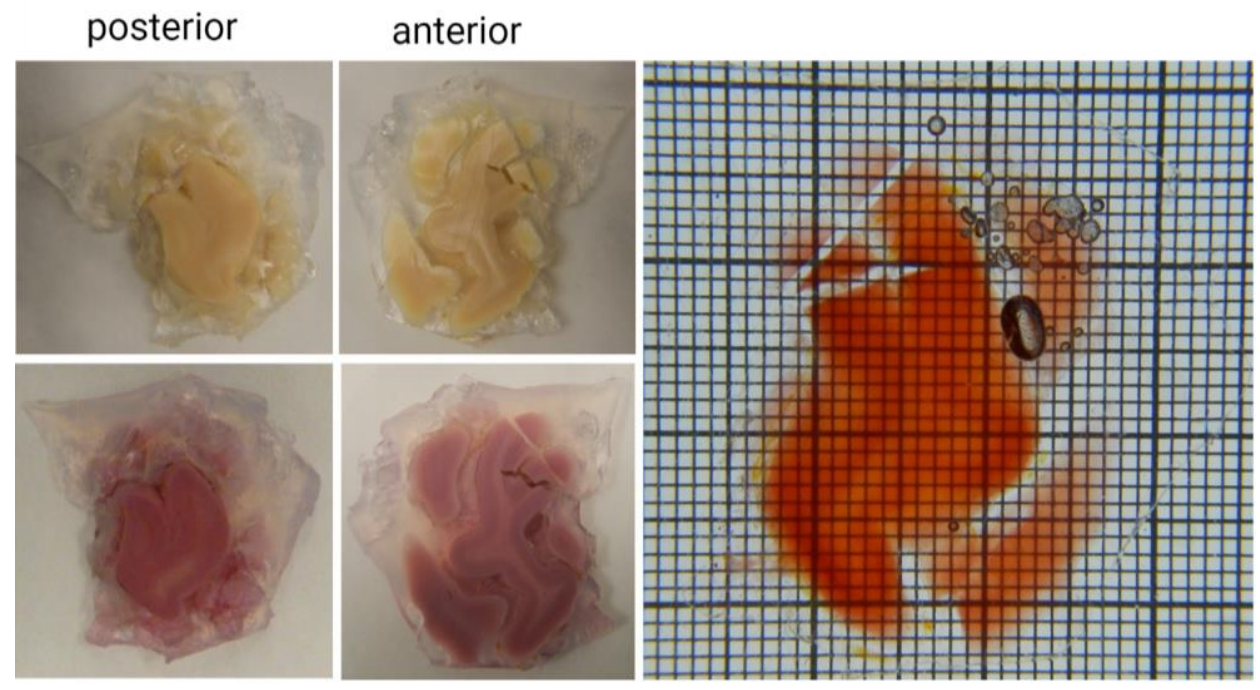

Supplementary Figure 8: Occipital lobe 2, slice 1 (posterior to anterior). Posterior views of the sample after bleaching (top) and staining (bottom) are shown in the left, anterior views on in the middle. The cleared and RI-matched sample is shown on the right (Grid: 1×1 mm smallest squares; $10 \times 10 \mathrm{~mm}$ bold squares). 


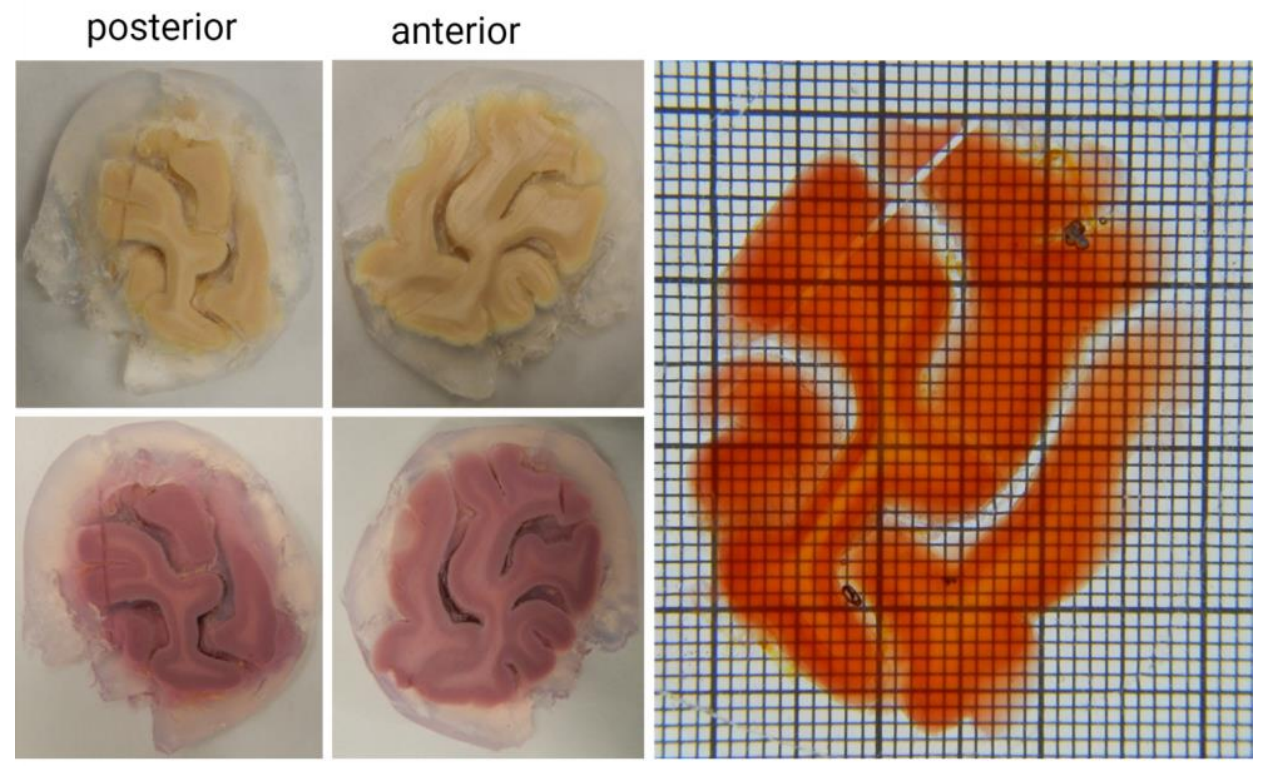

Supplementary Figure 9: Occipital lobe 2, slice 2 (posterior to anterior). Posterior views of the sample after bleaching (top) and staining (bottom) are shown in the left, anterior views on in the middle. The cleared and RI-matched sample is shown on the right (Grid: 1×1 mm smallest squares; $10 \times 10 \mathrm{~mm}$ bold squares). 


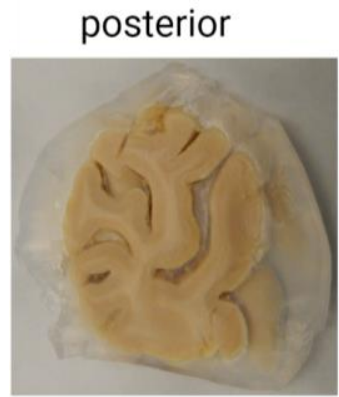

\section{anterior}
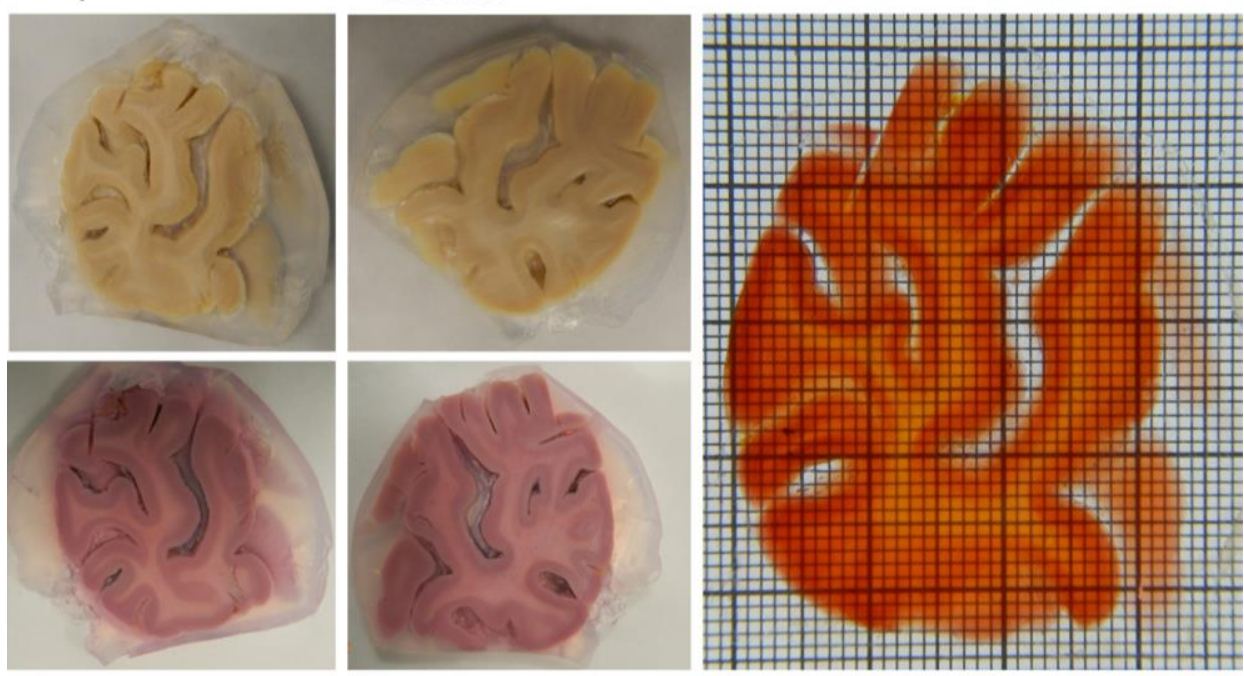

Supplementary Figure 10: Occipital lobe 2, slice 3 (posterior to anterior). Posterior views of the sample after bleaching (top) and staining (bottom) are shown in the left, anterior views on in the middle. The cleared and RI-matched sample is shown on the right (Grid: $1 \times 1 \mathrm{~mm}$ smallest squares; $10 \times 10 \mathrm{~mm}$ bold squares). 

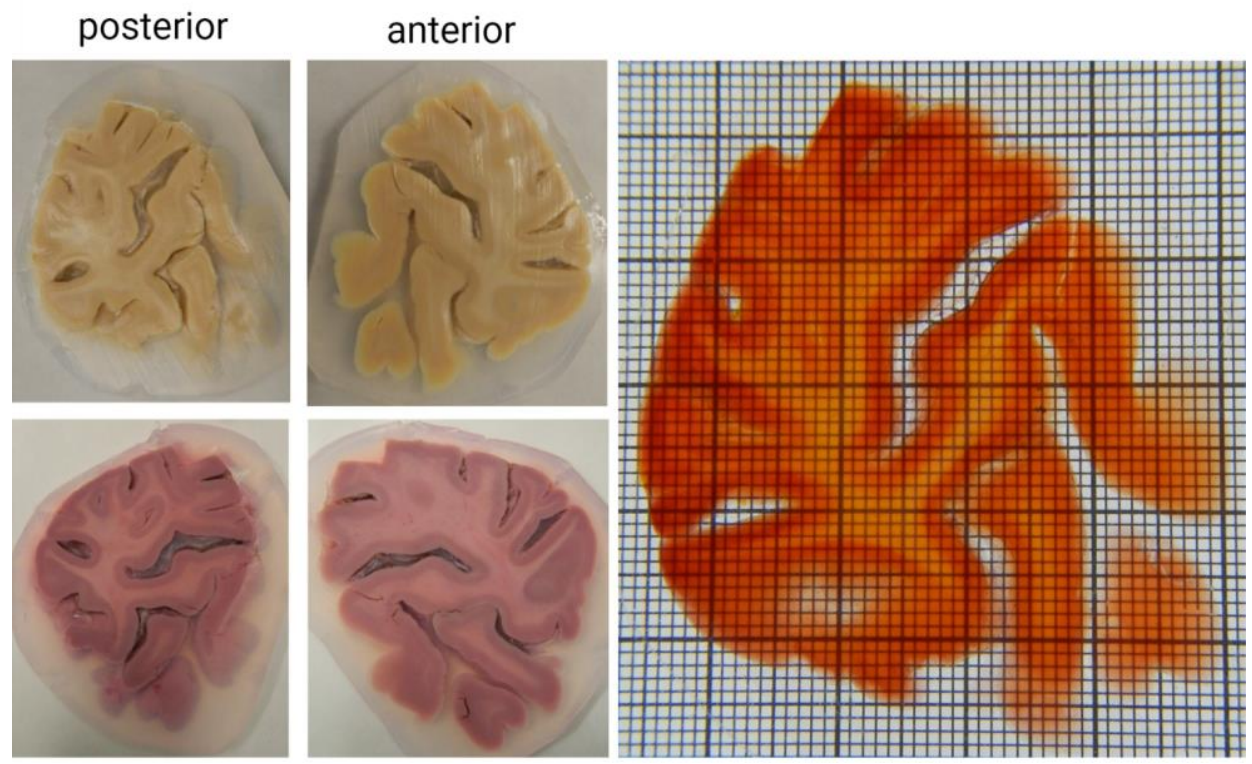

Supplementary Figure 11: Occipital lobe 2, slice 4 (posterior to anterior). Posterior views of the sample after bleaching (top) and staining (bottom) are shown in the left, anterior views on in the middle. The cleared and RI-matched sample is shown on the right (Grid: $1 \times 1 \mathrm{~mm}$ smallest squares; $10 \times 10 \mathrm{~mm}$ bold squares). 

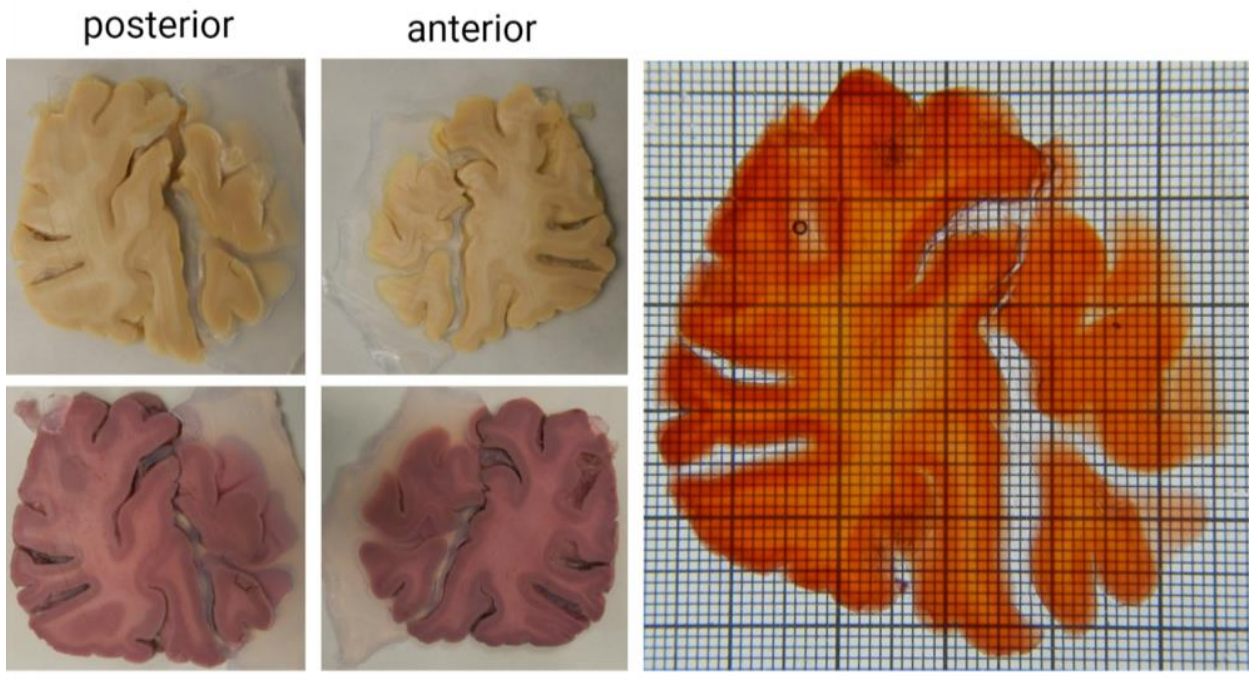

Supplementary Figure 12: Occipital lobe 2, slice 5 (posterior to anterior). Posterior views of the sample after bleaching (top) and staining (bottom) are shown in the left, anterior views on in the middle. The cleared and RI-matched sample is shown on the right (Grid: 1×1 mm smallest squares; $10 \times 10 \mathrm{~mm}$ bold squares). 


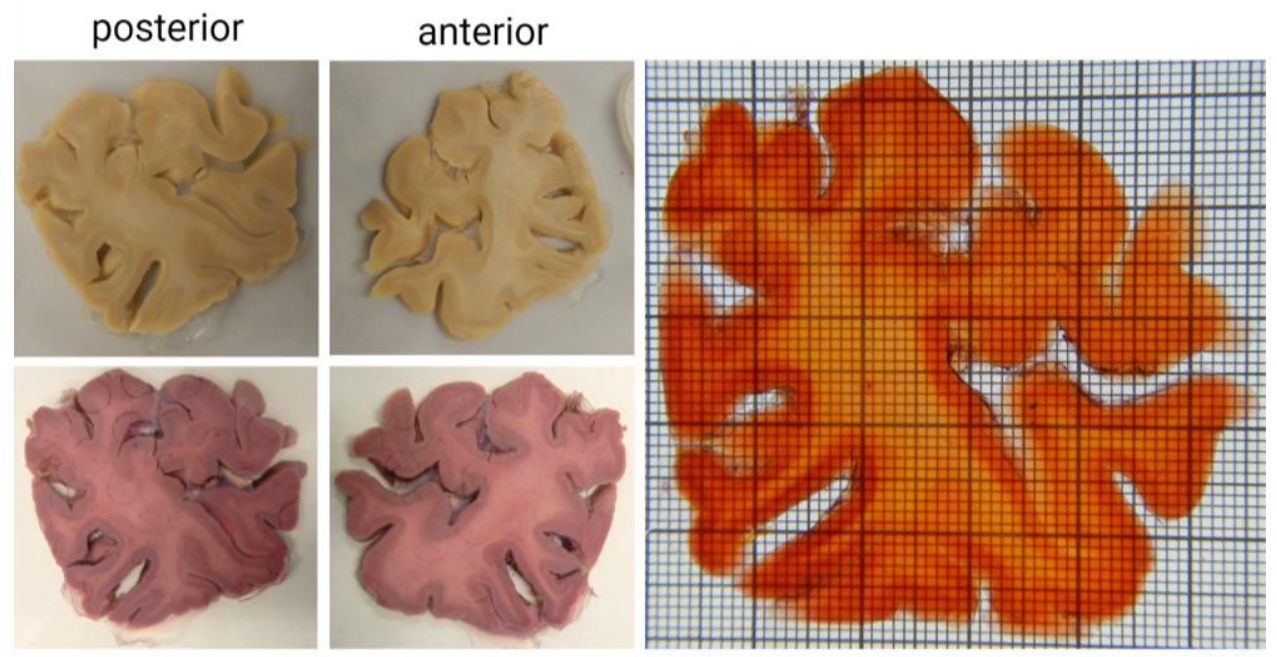

Supplementary Figure 13: Occipital lobe 2, slice 6 (posterior to anterior). Posterior views of the sample after bleaching (top) and staining (bottom) are shown in the left, anterior views on in the middle. The cleared and RI-matched sample is shown on the right (Grid: 1×1 mm smallest squares; 10×10 mm bold squares). 


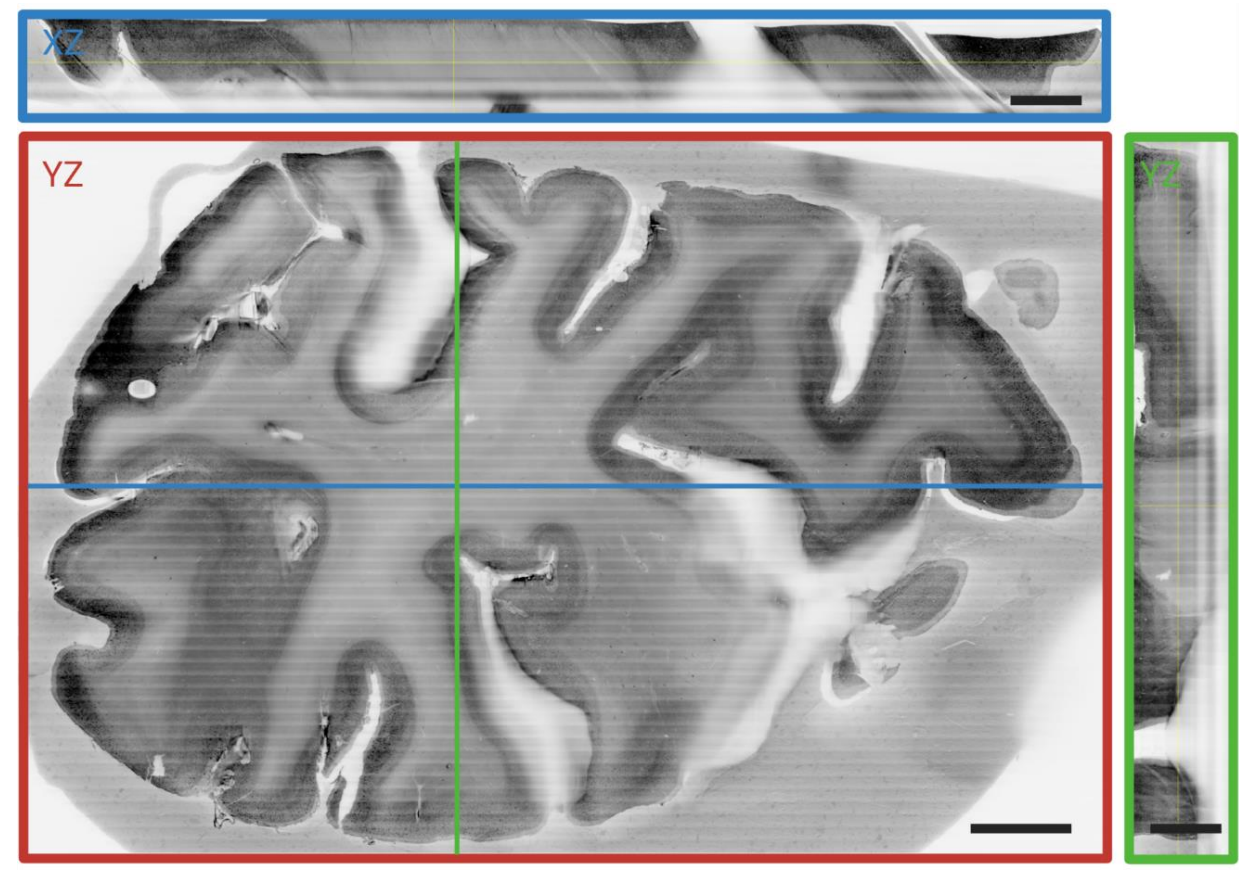

Supplementary Figure 14: Orthogonal views of the anterior most slice of occipital lobe 1. Single plane views across each axis to demonstrate the label penetration and quality. Scale bars: $Y Z: 5 \mathrm{~mm}$; $X Y$ and $X Z$ : $3.5 \mathrm{~mm}$. 


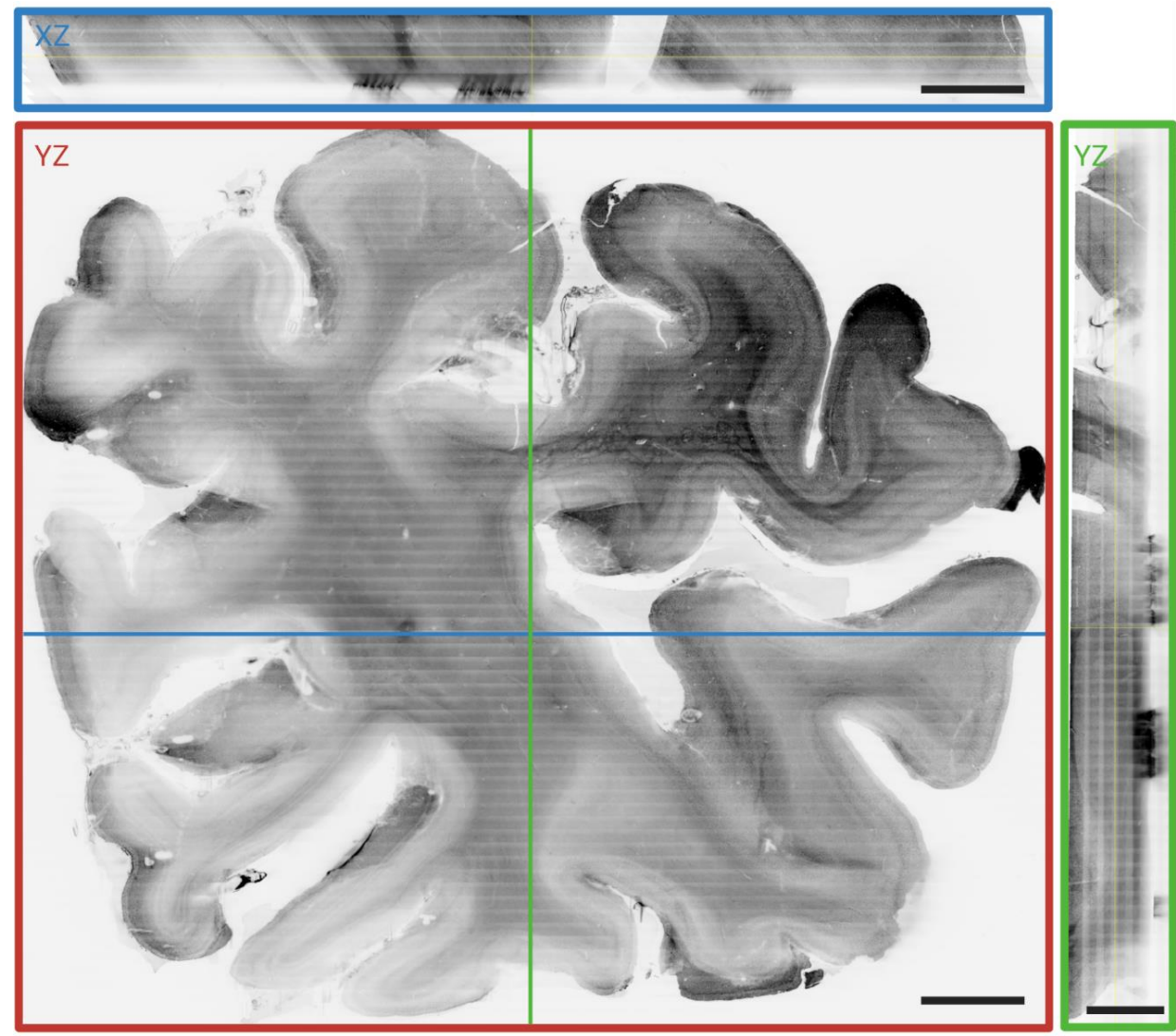

Supplementary Figure 15: Orthogonal views of the anterior most slice of occipital lobe 2 . Single plane views across each axis to demonstrate the label penetration and quality. Scale bars: $Y Z$ and $X Z: 5 \mathrm{~mm}$; $X Y$ : $3.5 \mathrm{~mm}$. 
Chapter 4 - High-throughput clearing 


\section{CHAPTER 5}

\section{cleared tissue dual Selective Plane Illumination Microscopy (ct-dSPIM) for fast mesoscopic imaging of large-scale} human tissues

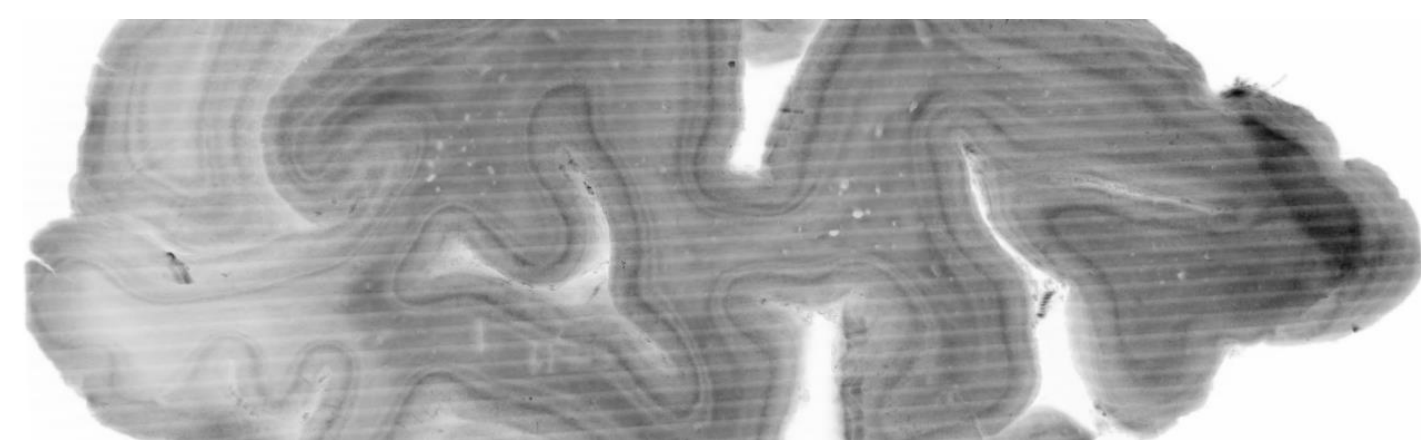

$3 \mathrm{D}$ rendering of an entire human occipital lobe slice imaged with the ct-dSPIM.

(in preparation)

Anna Schueth, Sven Hildebrand, Iryna Samarska, Shubharthi Sengupta, Annemarie Kiessling, Andreas Herrler, Axel zur Hausen, Alard Roebroeck 


\section{Abstract}

We present a novel light-sheet microscopy prototype, the clearedtissue dual view Selective Plane Illumination Microscope (ct-dSPIM). It is an implementation of Selective Plane Illumination Microscopy (SPIM) and allows for fast, high-resolution (nearly $1 \mu \mathrm{m}$ ), and largescale (many $\mathrm{cm}$ in lateral size and up to $5 \mathrm{~mm}$ thick) imaging of cleared tissue in 3D. We demonstrate the feasibility of ct-dSPIM imaging with $\sim 50 \times 35 \times 3 \mathrm{~mm}$ (i.e., $\sim 5 \mathrm{~cm}^{3}$ ) human brain and $\sim 40 \times 35 \times 5 \mathrm{~mm}$ (i.e. $\sim 7 \mathrm{~cm}^{3}$ ) prostate cancer samples, which have been processed with the MASH clearing and labelling protocol. Mesoscopic Fast Scans (MFSs) allow for relatively fast 3D overview scans of entire tissue blocks (MFS 16, $16.4 \mu \mathrm{m}$ isotropic resolution) or higher resolution overviews of large, selected ROIs (MFS 4, $4.1 \mu \mathrm{m}$ isotropic resolution). We were able to select and visualise ROIs around the border of human area V1 and V2, as well as image detailed prostate morphology in human prostate cancer biopsies. We show that ct-dSPIM imaging and MFS overviews are an excellent technique to assess entire MASH prepared large human tissue samples and enable multiscale investigations in large tissue volumes. The ct-dSPIM and multiscale MFS probing is suitable for future use with other clearing methods (with RI from 1.46 to 1.56) and a wide variety of other organs and tissues from humans and nonhuman mammals. 


\section{Introduction}

The visualisation and analysis of the microstructure of healthy and diseased organs and tissues (both ex vivo and post mortem) is crucial to investigate their function. Simultaneously, clinical studies and assessments based on these visualisations and analyses are essential for treatment decisions, for the development of drugs, and diagnostics, potentially influencing millions of lives. The ability to view human tissues in three dimensions (3D), with both microscopic resolution and large fields of view (FOVs), has the potential to considerably improve fundamental and clinical investigations. In the human brain, layered cortical cytoarchitecture stretches over the whole cortical sheet, blood vessels form large intricate networks ${ }^{1}$, and axons branch and extend over many centimetres and distribute across large areas ${ }^{2,3}$. Therefore, their structure is inherently multiscale, and their investigation requires both high-resolution imaging and large FOVs. In the prostate, tumours are characterised by multi-focality and a very heterogeneous morphology with diverse histo-morphological patterns in 3D throughout the entire sample. Therefore, a definitive diagnosis for prostate cancer requires histo-pathological verification of approx. $1 \mathrm{~mm}$ thick core-needle biopsies based on the Gleason Score grading ${ }^{4}$. This is very challenging as shown by the large interobserver variability, which in turn can lead to under- or overtreatment of patients ${ }^{5}$. It is difficult to identify low-grade prostate cancer and due to cost-inefficiency, complete cutups of prostate biopsy cores are rarely done. However, despite the clear advantages 
of large-scale microstructure visualisations, tissue samples in fundamental research and pathology are still mostly examined with conventional light-microscopes in paper-thin tissue sections $(5-100 \mu \mathrm{m})$, mounted on glass slides. This destroys most of the 3D structure of the organ, providing only limited 2D information over a small FOV and leads to real diagnostic problems e.g., a realistic risk of missing high-grade prostate tumours. Therefore, significant advances are needed towards high-speed and high-volume, 3D microscopy approaches with sufficient resolution to allow for the detection of crucial 3D features in their spatial context in large ( $\mathrm{mm}$ to $\mathrm{cm}$ size) tissue samples.

Optical tissue clearing, together with light-sheet fluorescence microscopy (LSFM) has started to be employed for 3D visualisation and examination of rodent and human tissue at micro- to mesoscale resolution $^{6-11}$. However, the application of cleared tissue light-sheet imaging to large archival (i.e., fixed with aldehyde fixatives) adult human brain samples in particular has been a major challenge because of the size of the samples and the difficulties of applying clearing, labelling and image processing protocols in the myelin-rich tissue. Previously, we published MASH (Multiscale Architectonic Staining of Human cortex), a novel clearing and labelling approach shown to be effective for up to $5 \mathrm{~mm}$ thick slabs of archival adult human brain tissue $^{12}$. Other research groups have demonstrated successful clearing of between $500 \mu \mathrm{m}$ and up to $1.5 \mathrm{~cm}$ think human brain samples ${ }^{13-17}$ and up to $1 \mathrm{~mm}$ thick human core-needle prostate 
samples ${ }^{18,19}$. A few investigations have shown the feasibility of the light-sheet microscopic application to optically cleared human brain samples ${ }^{13-16}$ with an actual imaged human brain sample size ranging from a $1 \mathrm{~mm}^{3}$ cube $^{7,16,20}$, to over $1.5 \mathrm{~mm}$ thickness $^{7}$ and up to $\sim 10 \mathrm{x} 14 \mathrm{x} 3 \mathrm{~mm}$ (i.e. $\left.0.44 \mathrm{~cm}^{3}\right)^{15}$. Although this shows that light-sheet technology and clearing protocols are evolving rapidly, 3D highspeed, large-scale cleared tissue imaging of prostate samples and human brain samples of many centimetres in lateral size and many $\mathrm{cm}^{3}$ in volume has not been reported yet.

Here, we present a novel light-sheet microscopy prototype, the cleared-tissue dual view Selective Plane Illumination Microscope (ctdSPIM). Our approach allows for 3D visualisation in large volumes, with high-speed and an adjustable speed-resolution trade-off, with the potential for single micrometre isotropic resolution. To show the feasibility of this method we describe ct-dSPIM imaging of human brain (occipital lobe) up to $\sim 50 \times 35 \times 3 \mathrm{~mm}\left(>5 \mathrm{~cm}^{3}\right)$ and prostate cancer (prostatectomy) samples up to $\sim 40 \times 35 \times 5 \mathrm{~mm}\left(\sim 7 \mathrm{~cm}^{3}\right)$ in size, processed with the MASH ${ }^{12}$ protocol. 


\section{Materials and Methods}

\section{Human brain samples}

Human occipital lobe samples were provided by Prof. Andreas Herrler of the Department of Anatomy at Maastricht University. The samples were taken from a human body donor (male; 98 years ; no known neuropathological diseases) of the body donation program of the Department of Anatomy and Embryology, Maastricht University. The tissue donors had given their informed and written consent to the donation of their body for teaching and research purposes as regulated by the Dutch law for the use of human remains for scientific research and education ("Wet op de Lijkbezorging"). Accordingly, a handwritten and signed codicil from the donor posed when still alive and well, is kept at the Department of Anatomy and Embryology Faculty of Health, Medicine and Life Sciences, Maastricht University, Maastricht, The Netherlands.

Brains were first fixed in situ by full body perfusion via the femoral artery. Under a pressure of 0.2 bar the body was perfused by 101 fixation fluid (1.8 vol \% formaldehyde, $20 \%$ ethanol, $8.4 \%$ glycerine in water) within 1.5-2 hours. Thereafter the body was preserved at least 4 weeks for post-fixation, submersed in the same fluid. Subsequently, brains were recovered by calvarial dissection and stored in $4 \%$ paraformaldehyde in $0.1 \mathrm{M}$ phosphate buffered saline (PBS) for 14-30 months. 


\section{Human prostate cancer biopsies}

All prostate resection and biopsy specimen were retrieved from the archive of the Department of Pathology Maastricht University Medical Centre (MUMC+) in the period between 2007 and 2015. For this publication 2 samples were derived from the same patient. The specimens were received for diagnostic purposes and processed according to the internal standard operating procedures. In short, the samples were initially fixated with $4 \%$ buffered formalin for 24 hours and further processed in the Vacuum Infiltrating Processor Tissue Tek VIP6 (Sakura Finetek USA, Inc, Torrance, CA, the USA), where the specimens were dehydrated by immersing in a series of ethanol solutions of increasing concentration until pure, water-free alcohol was reached. This step was followed by a clearing of the tissue in a Xylene solution, with consequent specimen infiltration paraffin. Finally, the specimens were embedded in paraffin according to the routine pathology diagnostic procedures in the HistoCore Arcadia Embedding Center (Leica Microsystems B.V., Amsterdam, the Netherlands).

Prostate samples had to be deparaffinised before clearing. For this purpose, samples were incubated for $3-7$ days in Xylene depending on sample size (for each sample a Xylene volume of about $200 \mathrm{ml}$ was used). Paraffin blocks were manually trimmed as much as possible before the incubation. After that, samples were rehydrated $1 \mathrm{~h}$ each in $100 \mathrm{ml}$ Xylene, 2x 100 \%, 70 \%, 50 \% ethanol (EtOH) and finally PBS. 
Rehydrated samples were kept in $4 \%$ buffered PFA solution until use. Clearing and labelling of the samples followed the same steps described below for the brain samples. During all these steps, prostate samples were kept in individual glass containers and incubated in at least $50 \mathrm{ml}$ of the respective solution. Containers were kept in a shaker at all times.

\section{Optical clearing and labelling}

All samples were MASH cleared and labelled as described in our previous publication $^{12}$. In short, samples were dehydrated for 1 h each in an aqueous mixture (v/v) of $20 \%, 40 \%, 60 \%, 80 \%$, and $100 \%$ methanol $(\mathrm{MeOH})$ at room temperature (RT) and $1 \mathrm{~h}$ in $100 \% \mathrm{MeOH}$ at $4{ }^{\circ} \mathrm{C}$. After that, samples were bleached overnight in a freshly prepared, chilled solution of $5 \% \mathrm{H}_{2} \mathrm{O}_{2}$ in $\mathrm{MeOH}$ at $4{ }^{\circ} \mathrm{C}$. Samples were then rehydrated for $1 \mathrm{~h}$ each in $80 \%, 60 \%, 40 \%, 20 \% \mathrm{MeOH}$ and permeabilised in phosphate buffered saline $+0.2 \%(\mathrm{v} / \mathrm{v})$ Triton X-100 $\mathrm{pH}$ 7.4. This was followed by an $1 \mathrm{~h}$ incubation in freshly filtered aqueous solution of $50 \%$ potassium disulfite $(\mathrm{w} / \mathrm{v})$ and 5 quick rinses followed by $1 \mathrm{~h}$ washing in distilled water. Labelling for cytoarchitecture was performed for 5 days in a solution of $0.001 \%$ neutral red in phosphate-citrate buffer (aka McIlvain buffer) ${ }^{21}$ at $\mathrm{pH} 4$. Samples were flipped after half the incubation time. After labelling, samples were washed 2x1h in McIlvain buffer $\mathrm{pH} 4$ and dehydrated for 1h each in $20 \%, 40 \%, 60 \%, 80 \%$, 2x $100 \% \mathrm{MeOH}$. Delipidation was performed overnight in $66 \%$ dichloromethane (DCM)/33\% $\mathrm{MeOH}$, 
followed by 2x 1 h 100 \% DCM. Finally, samples were incubated with ethyl cinnamate (ECi) as refractive index matching solution (RIMS). All steps were carried out at RT. For the incubation of multiple coronal slices of whole occipital lobes, a glass jar with a diameter of $8 \mathrm{~cm}$ was used, with spacers made out of polyethylene or polytetrafluorethylene, in order to provide compatibility with the organic solvents used during delipidation. To prevent the plastic spacers from leaving impressions on the tissue, pieces of filter paper were placed above and below the tissue. The glass jar was filled completely for each step described above (a volume of approx. $200 \mathrm{ml}$ ) and the solutions were constantly agitated with a magnetic stirrer during the entire processing.

\section{Imaging chambers}

For ct-dSPIM imaging, large customized ( $20 \times 15 \times 3 \mathrm{~cm}$, volume of approx. $900 \mathrm{~mL}$ ) imaging chambers (Fig. 1) were 3D printed in either ECi resistant watershed material (Somos®WaterShed XC 11122) or polypropylene (PP). The printing was performed either by SKM Rapid Modelling b.v. (Helmond, the Netherlands) via stereolithography (SLA) for the watershed prints or produced via Selective Laser Sintering (SLS) by Materialise NV (Leuven, Belgium) for the PP chambers. The imaging area of the chambers was equipped with a 178 x 127 x $1.2 \mathrm{~mm}$ glass slide (Ted Pella Inc., Redding, US) as a bottom to decrease light reflections and glued with pure silicone sealant. All samples were glued onto the glass slide in the 3D printed imaging chamber with hot 
glue (Rapid AB, Hestra, Sweden). Then, the imaging chamber was filled with approx. $500 \mathrm{ml}$ ECi solution (RI 1.56), depending on sample size.
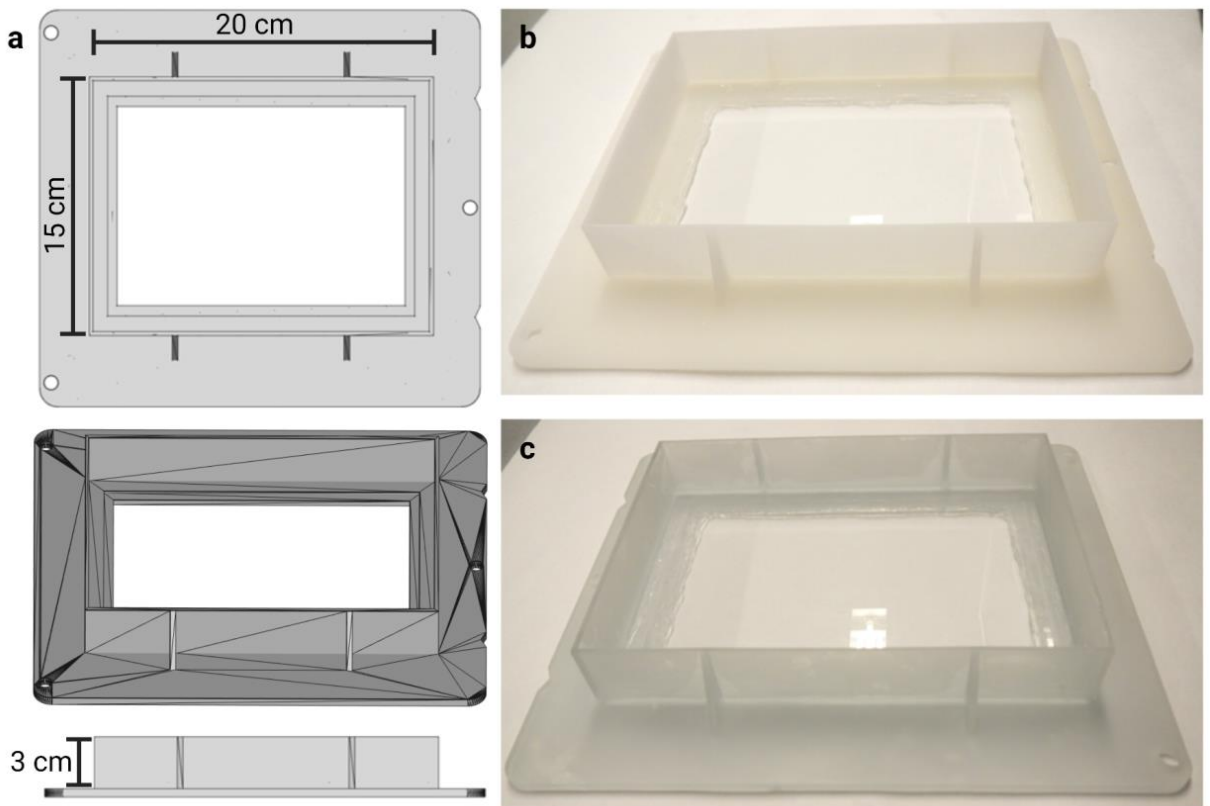

Figure 1: 3D printed imaging chambers for large cleared samples. a) 3D rendering of the larger imaging chamber used routinely on the ct-dSPIM set-up. The chambers have a volume on 0.9 I $(20 \mathrm{~cm} \times 15 \mathrm{~cm} \times 3 \mathrm{~cm})$. Chamber is shown in top view (top), oblique (middle), and side view (bottom). b) Chamber prototype printed with SLS in PP. c) Chamber prototype printed with SLA in Somos ${ }^{\circledR}$ WaterShed $X C$ 11122. Samples are mounted on top of glass slides, which are sealed with silicon to prevent the chamber from leaking RIMS.

\section{ct-dSPIM light-sheet microscope set-up}

The ct-dSPIM was co-development with Applied Scientific Instrumentation Inc. (ASI; Eugene, US), derived from the diSPIM (dual 
view inverted Selective Plane Illumination Microscopy) system ${ }^{22}$. An optical schematic of the ct-dSPIM system is shown in Figure 2. The laser light-source (Coherent obis, laser line 552nm LS 40mW LASER SYSTEM: FIBER PIGTAIL: FC) has a single-mode fibre output with a numerical aperture (NA) of 0.12 . The emitted laser light is collimated and passes through an electronically tuneable (ETL) lens (C 60TUNELENS-100, ASI) into the light-sheet "scanner" (MM-SCAN-1.2, ASI). The tuneable lens allows the axial position of the beam waist at the sample to be electronically controlled. The scanner contains a $2 \mathrm{D}$ micro-electro-mechanical mirror (MEMS) which sweeps the Gaussian beam across the sample once per camera image to form a "virtual" or "digital" light-sheet. The other axis of the MEMS mirror is used to adjust the light-sheet coincident with the detection objective's focal plane. The scanning beam is relayed to the back focal plane of the multi-immersion illumination objective (54-10-12, Special Optics/ Applied Scientific Instrumentation (ASI).

Fluorescence is collected by an identical multi-immersion detection objective (\#54-10-12, Special Optics/ ASI) and results in $\sim 1 \mu \mathrm{m}$ resolution laterally. The objectives have a refractive index range from 1.33 to 1.56 and a working distance (WD) of $12 \mathrm{~mm}$. Both, the numerical aperture (NA) and effective focal length (EFL) vary with refractive index, but for ECi the NA is $~ 0.43$, the EFL is $11.2 \mathrm{~mm}$, and the magnification is 17.9x. The maximum imaging depth is $5 \mathrm{~mm}$ and is limited by the physical clearance of the two objectives. The objectives 
are held by ASI-made mechanics including manual fine adjustments for co-aligning the two objectives (SPIM-DUAL-K2, ASI).

The excitation and detection paths are combined on a polychroic mirror (ZT488/543/635rpc-UF2, Chroma) and a motorized filter wheel (FW) (FW-1000-8, ASI) with three emission filters (ET519/26m; ET576/31m; ET655lp, Chroma). The filtered fluorescence is focused onto a 2048×2048 pixel scientific Complementary Metal-OxideSemiconductor (sCMOS) camera (ORCA-Flash4.0 V2, Hamamatsu) by a tube lens (C60-TUBE-B, ASI; $\mathrm{f}=200 \mathrm{~mm}$ ). The tube lens provides a Nyquist sampling of $\sim 0.38 \mu \mathrm{m} /$ pixel, with a horizontal field of view of $\sim 0.77 \mathrm{~mm}$ over the 2048 pixels of the camera.

Image strips are collected with a combination of stage-scanning and lateral/vertical tiling using a motorized XY stage (MS-8000, scanoptimized) and motorized $\mathrm{Z}$ actuators (Focusing Translation Platform (FTP-2050), ASI). The acquisition speed is $>10^{8}$ voxels $/ \mathrm{sec}$. The stagescanning firmware emits an internal (time-to-live) TTL trigger which ensures the reproducible start positioning $(<1 \mu \mathrm{m})$ of each image strip. An ASI tiger controller (TG-1000) controller contains control electronics for the motorized stages, MEMS mirror, tunable lens, and camera and laser triggers. It synchronizes all these elements with subms precision during each image strip based on the initial stagescanning trigger. 
a
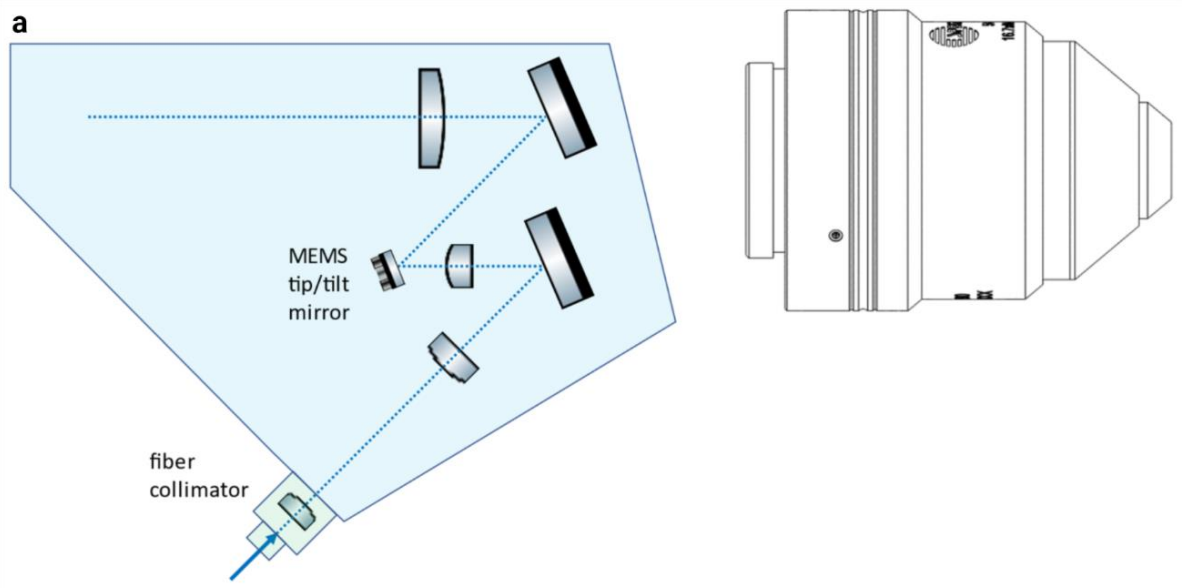

b

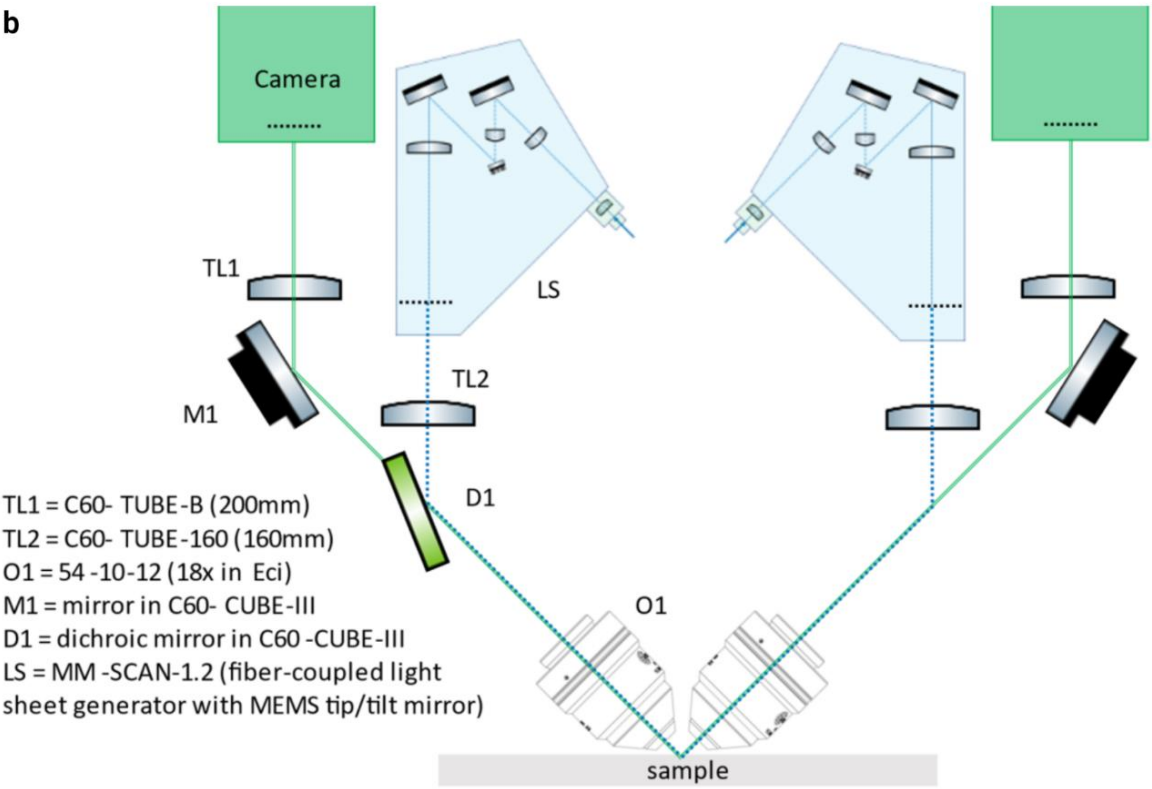

Figure 2: Schematic depiction of the ct-dSPIM setup. a) The emitted laser light is collimated and passes through an electronically tunable (ETL) lens (C60-TUNELENS-100, ASI) into the light-sheet "scanner" (MM-SCAN-1.2, ASI). The tunable lens allows the axial position of the beam waist at the sample to be electronically controlled. Legend continued on the next page. 
Figure 2: Continued. The scanner contains a 2D MEMS mirror, which sweeps the Gaussian beam across the sample once per camera image to form a "virtual" or "digital" light-sheet. The other axis of the MEMS mirror is used to adjust the light-sheet coincident with the detection objective's focal plane. b) The imaging paths are the two possible light paths (path $A$ and $B$ ). For each path, the scanner is on one side, and the imaging piezo and camera are on the opposite side. The light travels through the different components, such as the dicroic mirror and emission filter, as depicted (excitation paths: blue dotted line; emission paths: solid green line). The filtered fluorescence is focused onto a $2048 \times 2048$ pixel sCMOS camera (ORCA-Flash4.0 V3, Hamamatsu) by a tube lens (C60-TUBE-B, ASI; $f=200 \mathrm{~mm}$ ).

The microscope is controlled by $\mu$ Manager 1.4 , a free open-source microscope control software ${ }^{23}$. The ASI diSPIM plug in is used to align the microscope and to setup and perform acquisitions. The stage control plug in is used to make static ETL adjustments.

\section{Image acquisition}

All MASH-NR stained samples were imaged with the $552 \mathrm{~nm}$ laser line at $1 \mathrm{~mW}$ (OBIS) and with an exposure time of $10 \mathrm{~ms}$. We have performed Mesoscopic Fast Scans (MFS), which are 3D datasets of an entire tissue block at $16.4 \mu \mathrm{m}$ (MFS 16), or a region of interest at $4.1 \mu \mathrm{m}$ (MFS 4 ) or at near $1 \mu \mathrm{m}$ (MFS 1) isotropic mesoscopic sampled resolution after deskewing, limited by axial resolution and slice step length (not the lateral resolution limits) and acquired with a speed limited only by maximum stage scanning-speed (in the case of MFS 16). The slice steps 
are $11.60 \mu \mathrm{m}$ (MFS 16), $2.90 \mu \mathrm{m}$ (MFS 4) and $0.725 \mu \mathrm{m}$ (MFS 1), which lead to isotropic MFS resolutions after deskewing through the $\sqrt{2}$ scaling factor related to the 45 degree oblique sheet orientation with respect to the stage scanning axis.

\section{Data processing and visualisation}

The datasets were further downsampled as follows: For MFS16, 16x in plane (32x32 pixels), and for MFS 4 4x (128x128 pixels), to match the step size of the microscope and produce an isotropic dataset of $16.4 \mu \mathrm{m}$ (MFS 16) or $4.1 \mu \mathrm{m}$ (MFS 4) isotropic after deskewing. The downsampling as well as the stitching and deskewing of the downsampled data was performed with the Fiji ${ }^{24}$ PlugIn BigSticher ${ }^{25}$. $3 \mathrm{D}$ visualisation and cell count of volume stacks were done with arivis Vision4D software, version 3.4.0. For this purpose, a stack with the highest resolution (MFS1) acquired in human brain area V2 and covering layers I - V, was used. The deskewed stack was processed with an automated pipeline in Vision4D. The deskewed raw data was first filtered with the morphology filter option and cells were segmented with the blob finder segmentation method. To derive cell numbers per layer, 3 spherical regions of interest (ROIs) of $100 \mu \mathrm{m}$ diameter were manually placed into layers I, II-IIIa, IIIb, IV, and V respectively, by viewing the dataset in $3 \mathrm{D}$ and rotating it with the layers oriented perpendicular to the viewing angle. The volume was then clipped to a thin maximum intensity projection (MIP) to give a better impression of the layers. These ROIs where then used to create 
compartments in which only those segmented structures contained fully within the ROI borders were included. Features derived from these compartmentalised segments were then extracted into itemised .csv tables. To visualise the isotropic resolution of the lower resolution MFS 16 and MFS 4 datasets, orthogonal views were created in FIJI by reslicing the data and creating MIPs for each axis. Videos were created both with FIJI as well as with Vision4D. Figures were created with biorender (https://www.biorender.com). 


\section{Results}

\section{ct-dSPIM light-sheet microscope prototype}

The ct-dSPIM light-sheet imaging set-up is a flexible and easy-to-use implementation of Selective Plane Illumination Microscopy (SPIM) that allows for dual views (d) of large cleared tissue (ct) samples up to $5 \mathrm{~mm}$ thick (Fig. 2), derived from the diSPIM system ${ }^{22}$.

One key component of the ct-dSPIM are the two dipping multiimmersion objectives with a WD of $12 \mathrm{~mm}$ and a RI of 1,33-1,56, arranged in a $45^{\circ}$ angle above the sample mounted in the chamber. That is, two objectives are placed at right angles towards each other above the sample, mounted horizontally in the imaging chamber, with each objective having a $45^{\circ}$ inclination towards the sample. A light sheet is created from one objective and imaged using the other objective. A stack of images is collected by moving the light sheet through the sample; in the case of ct-dSPIM, the sample is normally moved through a stationary light sheet using the XY stage. For some applications, the 3D information from a single view or stack is sufficient. However, for the large sample imaging targeted here, extensive tiling is used into $\mathrm{y}-\mathrm{z}$ mosaic acquisitions, usually with long image stacks stage scanned in the $\mathrm{x}$ direction. As a dual view system, the ct-dSPIM has the further advantage that the role of the two objectives can be reversed to collect another stack from a perpendicular direction at the expense of twice the imaging time. The two datasets can be computationally merged to yield a 3D dataset with 
isotropic resolution; the usual problem of poor axial resolution is overcome by information from the other view ${ }^{22}$. However, since the emphasis here is on $>1 \mathrm{um}$ resolution fast large volume imaging, the dual view imaging mode is not employed in this work.

\section{Imaging of optically cleared large-scale human tissue samples}

We were able to demonstrate fast 3D imaging of large MASH cleared and labelled human brain (Fig. 3-5) and prostate cancer (Fig. 6) samples. Mesoscopic Fast Scans 16 (MFS 16) allowed for relatively high-speed (approx. 8-16 hrs, $\sim 1 \mathrm{~cm}^{3} / \mathrm{h}$ ) overview scans of an entire tissue block (up to $5 \mathrm{~cm} \times 3 \mathrm{~cm}$ in lateral size and $3 \mathrm{~mm}$ thick) and produced 3D data volumes with $16.4 \mu \mathrm{m}$ isotropic resolution (Fig. 3, 4, 6). Smaller ROIs were imaged with a resolution of $4.1 \mu \mathrm{m}$ isotropic for the human brain samples (Fig. 5). We present and discuss these results in turn.

\section{Human brain samples}

Human brain occipital lobe slices (Fig. 3-5) were taken approx. $6 \mathrm{~mm}$ anterior to the occipital pole (third $3 \mathrm{~mm}$ thick section in posterior to anterior direction; see Fig. 3 a and Suppl. Video 1) and the consecutive anterior slice ( $\sim 9 \mathrm{~mm}$ anterior to the pole) of same occipital lobe (Fig. $3 \mathrm{~b}$ and Suppl. Video 2). The dark discolourations in the top of the slice likely resulted from post mortem accumulation of blood at the back of the head. Stained slices clearly show the stripe of Gennari in V1 (Fig. 3, indicated by arrows). The morphology of the MASH cleared sample is 
well preserved after dehydration, delipidation and refractive index (RI) matching, and both grey as well as white matter become highly transparent.

We used the occipital lobe samples shown in Fig. 3 for ct-dSPIM MFS imaging. First, we performed an overview MFS 16 scan of the complete sample (see Fig. 3 and 4, and Suppl. Video 3). Figure 3 shows the 3D reconstruction of both slices with an MFS 16 data volume at $16.4 \mu \mathrm{m}$ isotropic resolution. The scanning direction can be recognized as the direction of the long imaging stacks or stripes (each $0.7 \times 0.7 \mathrm{~mm}$ deep and wide and many centimetres long), which are tiled in the remaining two directions to provide full 3D sample coverage. The densely stained layers can be easily distinguished even at low magnification in the large FOV overview scans. 


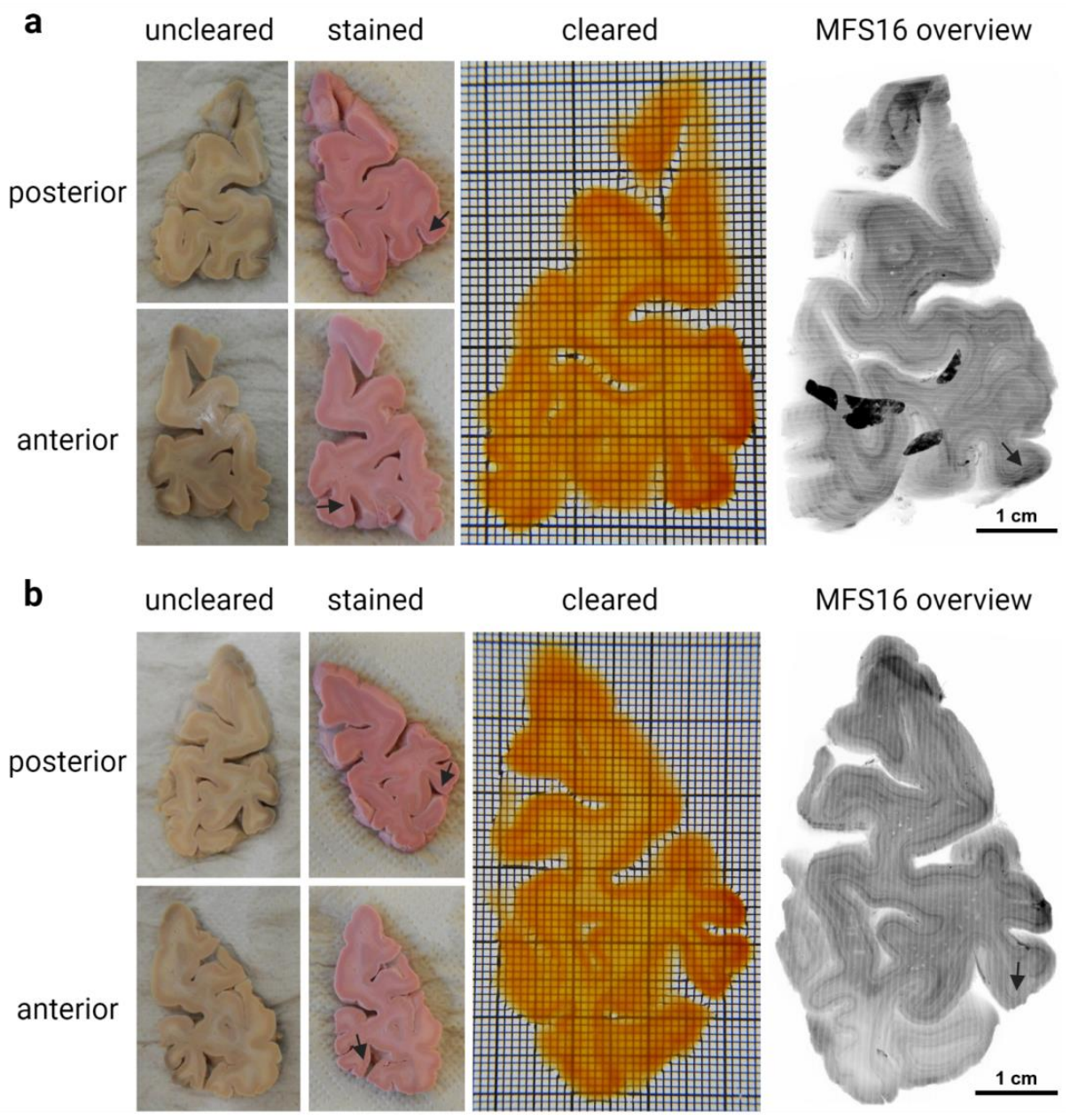

Figure 3: MASH processing of $3 \mathrm{~mm}$ thick human occipital lobe sections. a) Sample taken approx. $6 \mathrm{~mm}$ anterior to occipital pole (third $3 \mathrm{~mm}$ thick section in posterior to anterior direction). From left to right: Unstained sample from both sides. The dark discolourations in the top of the slice resulted from post mortem accumulation of blood in the back of the head. Stained slices clearly show the stripe of Gennari in V1 (arrows). Legend continued on the next page. 
Figure 3: Continued. a) Sample taken approx. $6 \mathrm{~mm}$ anterior to occipital pole (third $3 \mathrm{~mm}$ thick section in posterior to anterior direction). From left to right: Unstained sample from both sides. The dark discolourations in the top of the slice resulted from post mortem accumulation of blood in the back of the head. Stained slices clearly show the stripe of Gennari in V1 (arrows). The morphology of the cleared sample is well preserved after dehydration, delipidation and RI-matching, and both grey as well as white matter become highly transparent (grid: smallest squares 1x1 mm, bold squares 10x10 mm). $3 \mathrm{D}$ reconstruction of the entire slice at $16,4 \mu \mathrm{m}$ isotropic resolution with an MFS 16 acquisition. Densely stained layers are easily recognizable even at low magnification/large FOV overviews. b) Consecutive anterior slice of same occipital lobe. panels as described for a).

In figure 4 , both volume renderings and orthogonal views of $50 \mu \mathrm{m}$ MIPs are shown to better visualise the isotropic resolution obtained with MFS acquisitions. The different stage-aligned acquisition axes are indicated in green (XZ), red (YZ), and blue (XY) respectively. The $\mathrm{XY}$ view shows the tiling of the long acquisition stacks in the volume's $\mathrm{Z}$ direction (corresponding to the $\mathrm{X}$-axis of the stage during the acquisition). The orthogonal views also highlight the label penetration and quality throughout the entire $3 \mathrm{~mm}$ thick sample. 


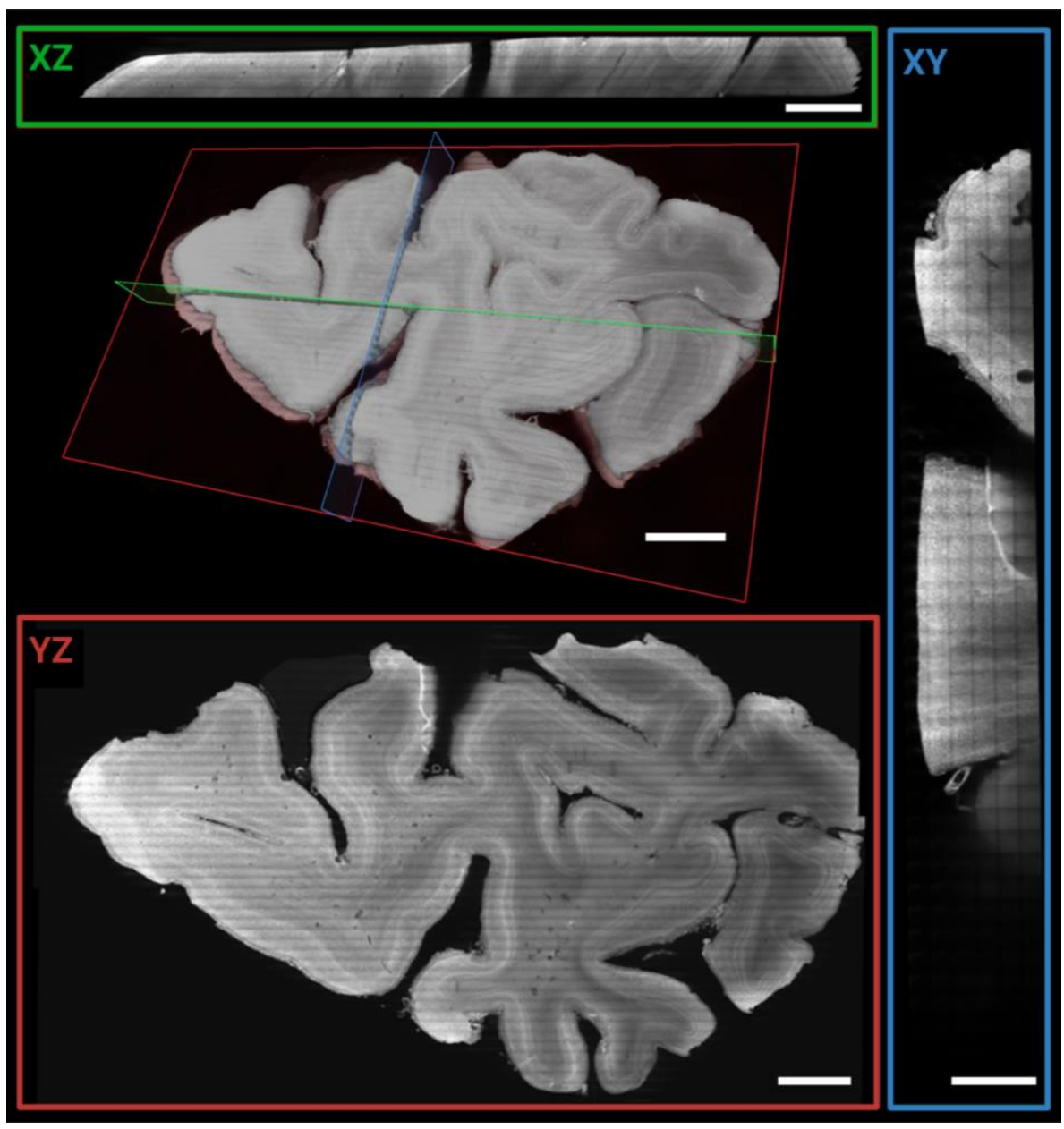

Figure 4: MFS 16 overview scan of entire occipital lobe sample. 3D rendering of $16.4 \mu \mathrm{m}$ isotropic data shows layered cytoarchitecture of the human visual cortex. Orthogonal views of $50 \mu \mathrm{m}$ MIP (XY: green, XZ: red, YZ: blue) highlight the labelling and imaging quality throughout the entire $3 \mathrm{~mm}$ thick sample. Scale bars: $5 \mathrm{~mm}$ for $\mathrm{XZ}, \mathrm{YZ}$ and volume rendering respectively and $2.5 \mathrm{~mm}$ for XY plane. 
After the overview MFS 16, a higher resolution $4.1 \mu \mathrm{m}$ isotropic resolution MFS 4 scan was performed in a ROI close to the V1/V2 border (Fig. 5). The orthogonal views of $50 \mu \mathrm{m}$ Maximum Intensity Projections (MIP) are indicated by green (XY), red (XZ), and blue (YZ) panels respectively and clearly show cortical layers independent of the orientation. The higher magnification insets (XY: yellow, XZ: magenta, YZ: cyan) are a ROI of the MIP and qualitatively demonstrate near isotropic resolution (isotropically sampled at $4.1 \mu \mathrm{m}$, but anisotropic optically through a $\sim 8 \mu \mathrm{m}$ thick lightsheet) and high image and labelling quality even deep within the sample (Fig. 5). 


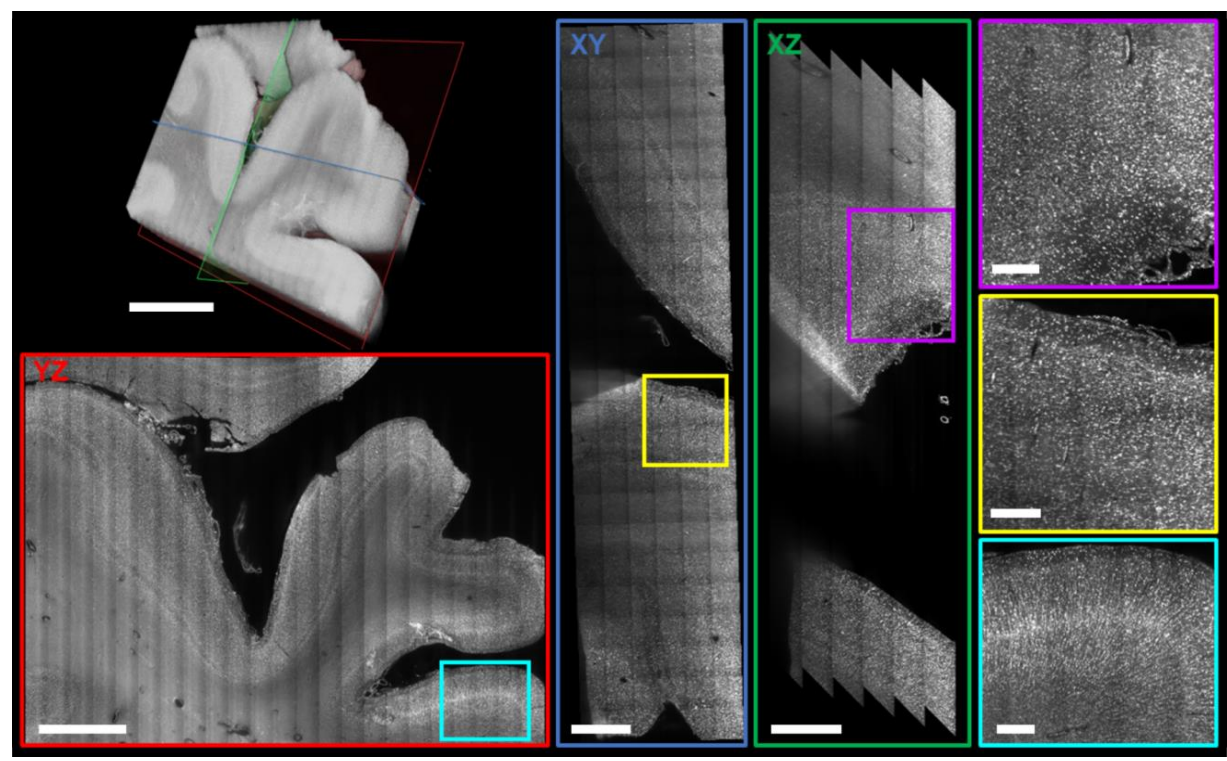

Figure 5: Higher resolution MFS 4 scan of human occipital lobe sample. 3D rendering of $4.1 \mu \mathrm{m}$ isotropic data of a ROI in the vicinity of the human primary visual cortex. Orthogonal views of $50 \mu \mathrm{m}$ MIPs (XY: blue, XZ: green, YZ: red) clearly shows cortical layers independent of the orientation. Jagged edges in XZ view result from deskewing when the edge of the acquisition is inside the tissue. Magnified ROls of the MIP (XY: yellow, XZ: magenta, YZ: cyan) demonstrate qualitatively the isotropically sampled resolution and high image and labelling quality even deep within the sample. Scale bars: Volume rendering, $X Y$, and $Y Z$ plane $3 \mathrm{~mm}$; XZ: $1.5 \mathrm{~mm}$; magnified ROIs $0.5 \mathrm{~mm}$, respectively.

\section{Prostate cancer biopsies}

For the first time the MASH protocol with MASH-NR labelling has been successfully applied to large prostate cancer samples (Fig. 6 a-c; 6 e-g). Prostate samples were formalin-fixed and paraffin-embedded (FFPE) samples. We therefore demonstrate the feasibility for the 
application of MASH not only on a different tissue type, but also on FFPE material. Moreover, we were able to show the feasibility of novel high-speed (imaging duration: approx. 5-7 hours, $\sim 1 \mathrm{~cm}^{3} / \mathrm{h}$ ) MFS 16 ctdSPIM imaging on large MASH cleared and MASH-NR labelled prostate cancer samples. MIPs over approx. $50 \mu \mathrm{m}$ from MFS $16 \mathrm{ct}-$ dSPIM overview scans are shown in an inverted grey scale (Fig. $6 \mathrm{~d}, \mathrm{~h}$ ). These MIPs show in plane views of the complete sample surface of the resliced data at $16.4 \mu \mathrm{m}$ isotropic resolution. The large mesoscopic overviews allow for the anatomical description of prostate tissue morphology and the indication of cancerous regions (indicated by red circles). Different layer zones and compartment of the prostate gland could be detected (Fig. $6 \mathrm{~d}, \mathrm{~h}$ ), namely the fibro muscular stroma (AFS), the central zone $(\mathrm{CZ})$, the peripheral zone $(\mathrm{PZ})$, the transition zone (TZ) and the urethra (PUV). 

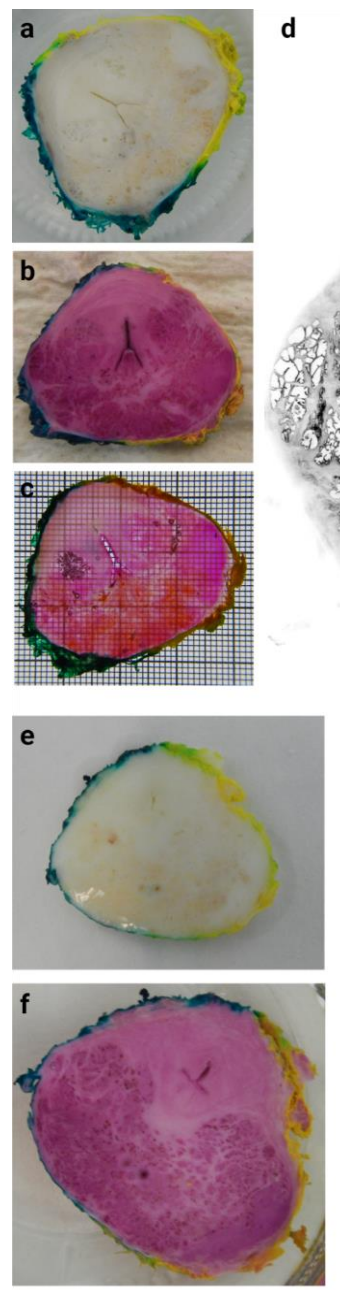

h
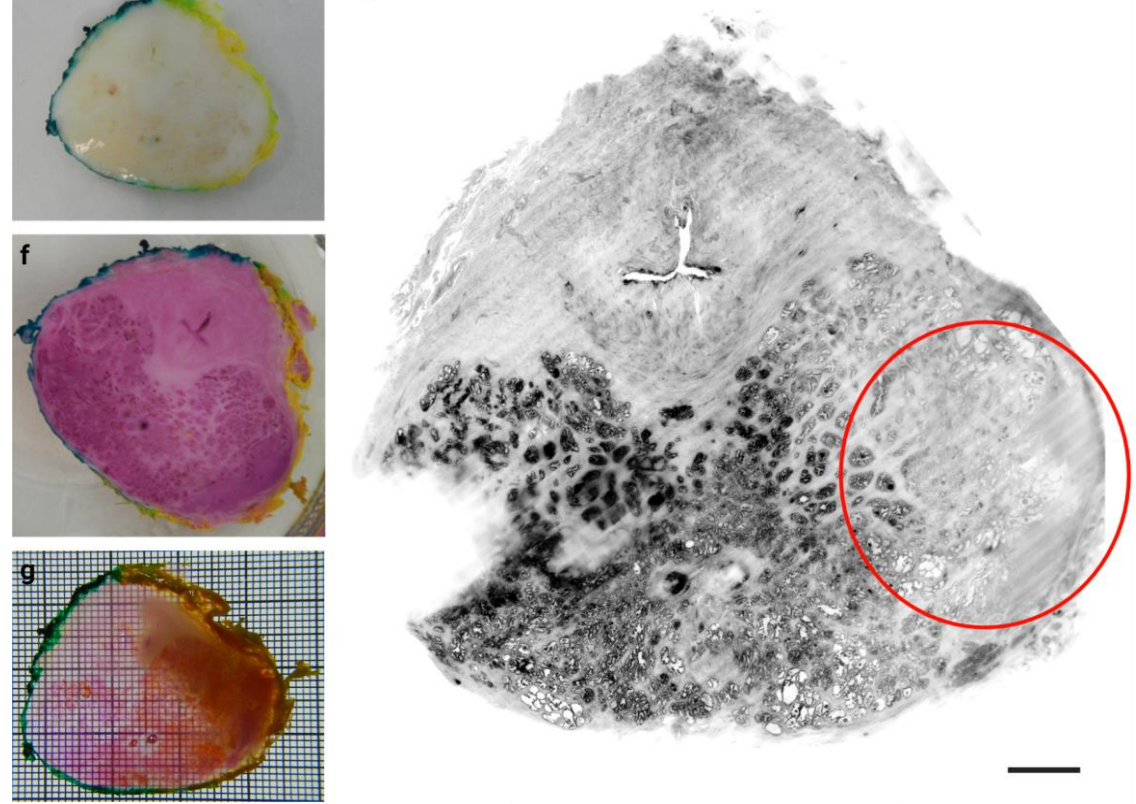

Fig. 6: Prostate cancer samples cleared and labelled with MASH, imaged with ct-dSPIM MFS 16. Two samples are shown (prostate sample $1 \mathrm{a}-\mathbf{d}$ and prostate sample $2 \mathrm{e}-\mathrm{h}$ ), which were both derived from the same patient. Legend continued on the next page. 
Fig. 6: Continued. Samples are shown at various stages during the processing pipeline on the left side: After deparaffinisation and bleaching ( $a$ and $\mathbf{e}$ ), after staining ( $b$ and $\mathbf{f}$ ), and after RI-matching ( $\mathbf{c}$ and $\mathbf{g}$ ). On the right side, MIPs (from MFS 16 data sets) over approx. $50 \mu \mathrm{m}$ are shown ( $\mathbf{d}$ and $\mathbf{h}$ ) in inverted greyscale. Red circles indicate regions identified as cancerous. Abbreviations: $\mathrm{AFS}=$ anterior fibromuscular stroma, $\mathrm{CZ}=$ central zone, $\mathrm{PUV}=$ prostatic urethra and verumontanum, $\mathrm{PZ}=$ peripheral zone, $\mathrm{TZ}=$ transition zone. Scale bars: $3 \mathrm{~mm}$.

\section{Quantitative 3D data analysis: Cell counting}

A small brain sample volume from a single imaging stack obtained with the highest resolution (0.3625 $\mu \mathrm{m}$ pixel size in plane), containing layers I-V of human area V2 was processed for cell counting (Fig. 7). To this end, the data was first deskewed and an automated pipeline was created to filter and segment the cell bodies (Fig. 7 a-d). To count the segmented objects, three spherical ROIs of $100 \mu \mathrm{m}$ diameter were placed manually within the different layers (Fig. 7 e and Suppl. Video 3). The separation of layer II and IIIa could not be guaranteed and the results for these layers where thus pooled. Layer VI was not contained within the data volume and could thus not be included in the analysis. Segments with a volume smaller than $125 \mu \mathrm{m}^{3}$ were excluded from the analysis as those were considered too small to represent cell bodies. The total number of all segments contained within each ROI is shown in figure $7 \mathrm{f}$. As expected, the number of objects in layer I is lowest and granular layer IV has the highest density of cells. Surprisingly, the ROIs 
placed within layers II/IIIa and layer IIIb contain a similar number of segments, contrary to the qualitative impression of a lower cell density in the lower region on layer III. The low cell numbers in the layer $\mathrm{V}$ ROIs agrees well with the general impression of the cell density of this layer in the data volume (compare also Suppl. Video 4). To assess whether the morphological differences of neurons can be extracted from the segmented results, we compared the sphericity of the segments of all the ROIs within a particular layer (Fig. 7 g). The sphericity is indicated as a value between 0 and 1 , where 1 represents a perfect sphere. Our expectations were, that the high number of pyramidal neurons in the inner and outer pyramidal layers would have a lower sphericity and that this would be consequently reflected in the distribution of the sphericity between the different segment populations. Although there seems to be a trend towards less spherical segments in the layer III and V ROIs, as expected, no clearly distinguished difference could be observed between the current segment population sizes of the different layers. 

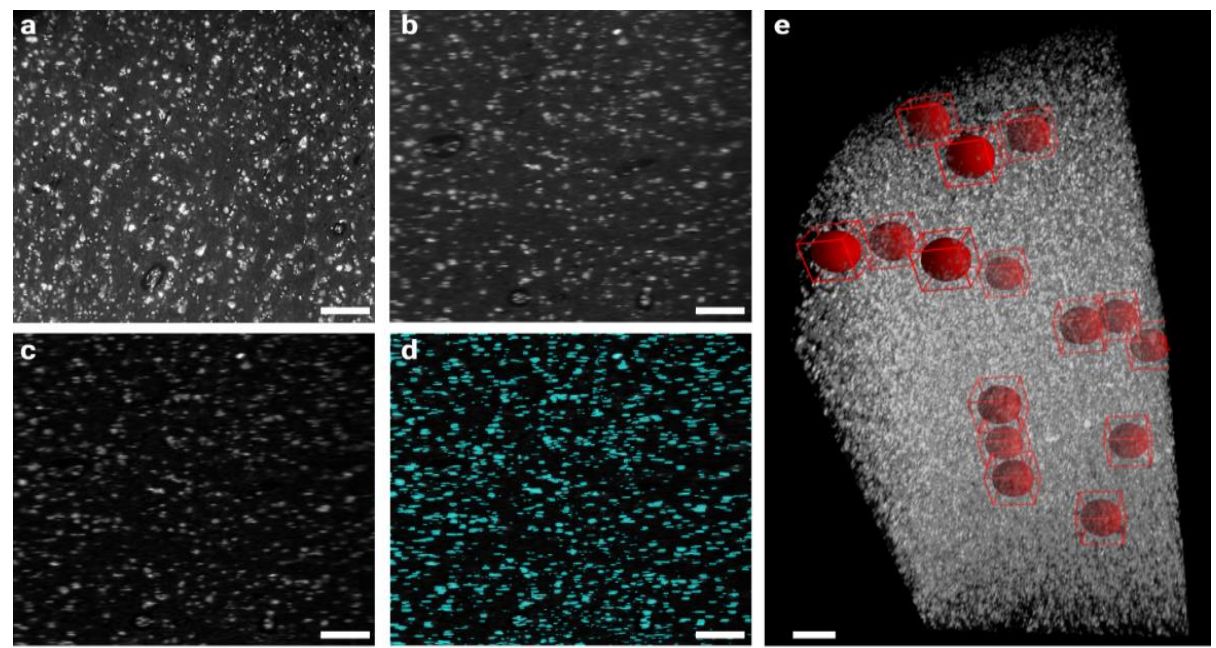

f
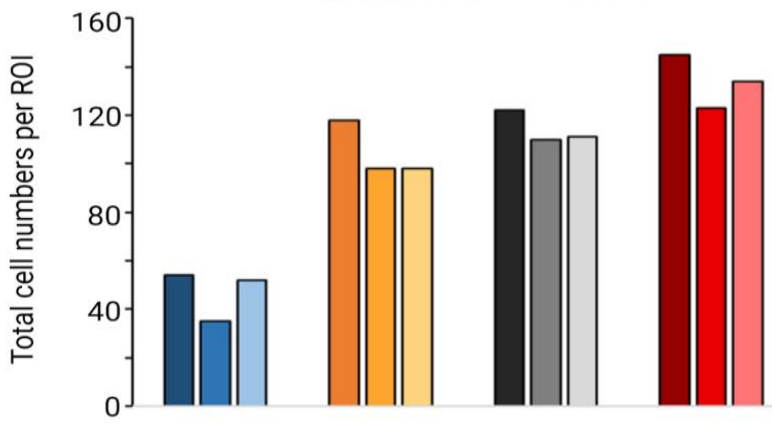

Layer I

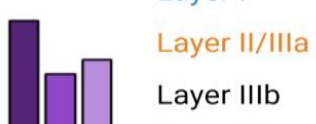

Layer IIIb

Layer IV

Layer $\mathrm{V}$

g

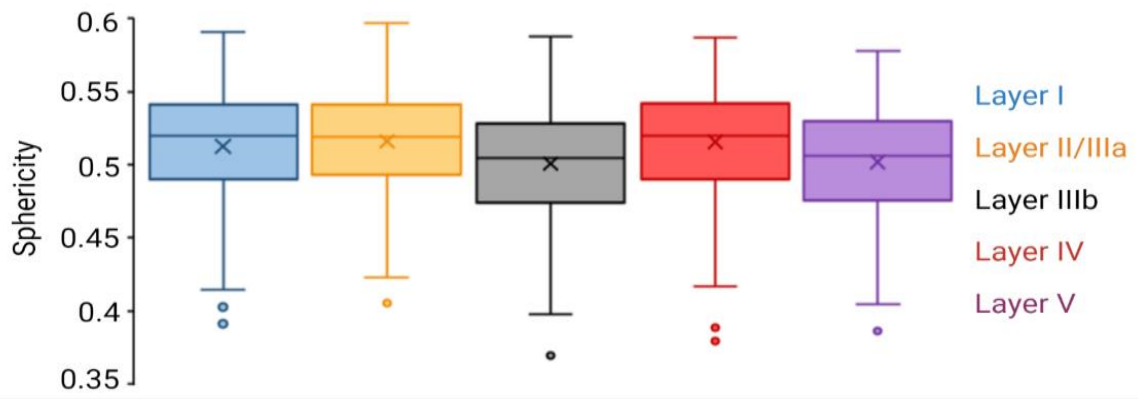

Figure 7: Segmentation and cell counting in a high resolution, single-view volume of human brain tissue. Single planes (XY plane of the image volume) are shown of the undeskewed raw data (a), the unprocessed data after deskewing (b) and preprocessing to remove noise (c). Legend continued on the next page 
Figure 7: Continued. The segmentation results for the same plane as in $\mathbf{b}$ and c are shown in d. For counting and analysis of the segmented objects, spherical ROls of $100 \mu \mathrm{m}$ diameter were placed in the volume (red spheres in e). The ROls were manually placed by viewing the clipped plane of the volume perpendicular to the cortical layers. Three ROls were positioned for each layer contained within the dataset. The total numbers of segments contained in each $\mathrm{ROI}$ is depicted in $\mathbf{f}$. The distribution of the sphericity of all segments within one layer is shown in $\mathbf{g}$ (line: median; $\mathrm{x}$ : mean sphericity). 


\section{Discussion}

We set-up and co-developed the worldwide first prototype of the cleared-tissue dual view Selective Plane Illumination Microscope (ctdSPIM), together with Applied Scientific Instrumentation (ASI). The ct-dSPIM allows novel fast $\left(>10^{8}\right.$ voxels/sec), 3D, high-resolution (near $1 \mu \mathrm{m}$ ) imaging of large-scale cleared tissue samples (several $\mathrm{cm}$ in lateral and up to $5 \mathrm{~mm}$ in depth). One key component of the ct-dSPIM are the two dipping multi-immersion objectives with a WD of $12 \mathrm{~mm}$ and a RI of 1,33-1,56, arranged in a $45^{\circ}$ angle above the sample mounted in the chamber. The entire system is a flexible and easy-touse light-sheet microscopy implementation.

\section{ct-dSPIM MFS imaging of MASH processed samples}

We were able to demonstrate the efficiency and feasibility of ctdSPIM imaging with both MASH prepared ${ }^{12}$ archival human occipital lobe (Fig. 3-5) and prostate cancer biopsies (Fig. 6). The MASH protoco ${ }^{12}$ with MASH-NR labelling was successfully applied to large FFPE prostate cancer samples for the first time (Fig. $6 \mathrm{a}-\mathrm{c} ; 6 \mathrm{e}-\mathrm{g}$ ). This does not only show the applicability of MASH to tissues other than brain, but equally important to FFPE samples. In the original publication, MASH has been applied to formalin-fixed archival human brain samples that were kept in $4 \%$ PFA until use. The use of MASH on FFPE material could massively increase its application in other domains, as FFPE tissue has been commonly used in research and therapeutic application for decades. Additionally, this material is more 
easily stored and widely available. The most important step in the modification of MASH towards FFPE tissue is the initial deparaffinisation in xylene, which must be sufficiently long to allow the complete dissolution of the paraffin in thick samples. Following our tissue processing, optical clearing and labelling pipeline, we have performed MFS scans of either an entire tissue block at $16.4 \mu \mathrm{m}$ isotropic resolution (MFS 16), or a region of interest at $4.1 \mu \mathrm{m}$ near isotropic resolution (MFS 4 ), limited only by the $\sim 8 \mu \mathrm{m}$ light sheet thickness in one direction. The acquisition speed of MFS scans is only limited by the maximum stage scanning- speed and currently reaches $\sim 1 \mathrm{~cm}^{3} / \mathrm{h}$. MFS 16 overview scans allowed for high-speed (approx. 8$16 \mathrm{hrs}$ ) visualisation of an entire prostate tissue block ( 40 × 30 × $5 \mathrm{~mm}$ ). MFS overview scans of human brain (occipital lobe) samples revealed that densely stained layers could be easily distinguished (Fig. 3) and MIPs demonstrated the labelling and imaging quality throughout the entire $3 \mathrm{~mm}$ thick sample (Fig. 4). The higher-resolution MFS 4 in a ROI in the vicinity of the human primary visual cortex make it possible to distinguish individual cells (Fig. 5, magnified panels).

Maximum Intensity Projections (MIPs) of prostate cancer MFS 16 scans allow for visualisation of 3D high-resolution prostate tissue morphology including cancerous regions (Fig. $6 \mathrm{~d}, \mathrm{~h}$ ). MFS 16 scans of prostate cancer biopsies has an average duration of 5-7 hours. Prostate cancer diagnostics, in particular of low-grade tumour is very challenging ${ }^{4}$ and the assessment of a complete cut-up would take 10 days, which is inefficient and expensive, and therefore almost never 
performed in standard-of-care clinical practice. Therefore, MFS ctdSPIM imaging opens up new avenues for future cancer diagnostics, in collaboration with oncologists and pathologists.

Although we used MASH prepared human and prostate samples as application cases for MFS acquisitions on the ct-dSPIM, this technique could potentially be extended to a variety of other human and non-human mammalian tissues as well.

\section{Cell segmentation and counting}

In general, our results seem to be in good agreement with numbers derived from earlier stereological work. Leuba and Garey ${ }^{26}$ found average cell numbers in supragranular layers of V2 of about 132 and 113 in infragranular layers respectively when converted to the ROI volumes used in this study of a $100 \mu \mathrm{m}$ diameter spherical volume. In comparison, the average cell number in all supragranular ROIs combined in the dataset presented here was 112 and the average cell number in layer $\mathrm{V}$ was 96. Unfortunately, the absence of layer VI prevents us from a better comparison with their infragranular numbers. Numbers in layer IV are lower in our case with 134 as compared to 227 for Leuba and Garey. This could possibly be explained by the relatively narrow width of this layer and its curvature in this particular dataset, leading to part of the ROI volumes being located in either layer III or V. However, in the dataset of Leuba and Garey, the granular layer showed the largest variance and the lowest count in one of their samples was 173 neurons. The fact that our 
numbers seem to be lower in general as compared to their estimates could be explained by their larger sample size and higher age range in the samples. Additionally, it should be noted that their method differs substantially, as their numbers were derived from stereological investigations of 2D sections, even when we use this as a gold standard reference. Furthermore, we applied very strict conditions in our cell counting pipeline, in which only segments contained fully within the borders of the spheres were taken into consideration. It remains to be seen whether the cell counts derived from our method within a 3D volume would show even better congruence, if a larger sample size were used. In this proof of principle, we analysed ROIs of a fixed size within a single volume of one tissue donor.

In future work, rather than analysing only sub-volumes of each layer, the entire layer within a dataset could be segmented and thereby a cell count of the whole layer generated. The cell counts and morphology analysis would be yet more robust when it could also be expanded to the analysis to include multiple samples from multiple subjects, which might give us insight into the source of the variance. Additionally, the implementation of a more sophisticated segmentation using machine learning ${ }^{27}$ could further improve the quality of the analysis. In our current work, the threshold-based segmentation included many smaller objects of only a few pixels in size, obviously too small to represent cells. These smaller objects were later excluded from the cell count and sphericity analysis, but the threshold for this is user dependent. In addition, objects of any shape 
were counted, whereas shape might reveal what type of cell it is, or whether it is a cell at all. A more advanced, deep-learning-based segmentation and categorisation would overcome these shortcomings. Here, we only compared absolute numbers and the sphericity. Future work could focus on extracting more features from the segmented structures. To disentangle the heterogeneous cell populations, multiple labels could be applied to differentiate e.g., excitatory and inhibitory neuronal populations in the different layers and derive a more detailed characterisation of the layer-specific ratios of excitation and inhibition in different brain areas. In principle, MASH is compatible with such antibody labels, which are specific to these neuronal subtypes. However, antibody labels will not penetrate as deeply into samples as the small molecule MASH labels with passive diffusion only and more sophisticated labelling approaches need to be developed for immunofluorescent staining of samples the size demonstrated here (for discussion, see Chapter 2, General Introduction and General Discussion, this thesis).

\section{Comparison to other approaches}

We demonstrated ct-dSPIM MFS imaging successfully and showed that we were able to overcome limitations of other existing light-sheet systems for very large human sample coverage ${ }^{18}$. The ct-dSPIM uses an oblique geometry with the objectives dipping directly into the imaging liquid, unlike other recently published set-ups ${ }^{18,28}$, in which the objectives are located beneath the sample. This geometry 
necessitates imaging through the glass bottom of the chamber, introducing potential aberration by RI-mismatches between sample, imaging liquid and glass slide. Additionally, the sample size is further limited by this geometry as WD is effectively lost by imaging through the glass. Other systems use a more standard (i.e. upright, not oblique) light-sheet geometry and use a novel way of generating the lightsheet itself to image very large samples ${ }^{11}$. But even in these systems, the lateral extent will ultimately be limited, as even in the most transparent samples, light scattering will occur to some degree and the image quality deteriorates. This can be avoided by an oblique setup such as the ct-dSPIM, leaving the lateral size of the sample limited only by the travel range of the microscope stage.

\section{Future perspectives}

Currently, we are establishing further MASH pipelines, such as the imaging and analysis of brain angio-architecture (see Chapter 3 of this thesis) which are also suitable for ct-dSPIM very large sample imaging. In the future, the entire pipeline from tissue processing (with MASH clearing and labelling) to ct-dSPIM MFS imaging and data analysis can be extended to larger parts of the brain and brain regions, such as the temporal lobe. As the lateral sample size can potentially be much larger in the current set-up, it would even be possible to image whole brain slices, provided a tissue-processing pipeline for samples of this size is established. Further hardware changes, such as a cylindrical lens (rather than digital laser-scanned) scanner, and stage tiling 
trajectory adjustments, such as a serpentine trajectory, could potentially further increase imaging speed 2- to 4-fold. This would open up a completely new scale in the investigation of healthy and diseased brain tissue, both ex vivo and post mortem. Other hardware modifications of the ct-dSPIM set-up, such as higher NA and magnification objectives, could potentially allow for an increase in resolution for imaging of e.g., dendritic trees and potentially dendritic spines. These future developments will allow us to image different structures and markers, in larger parts of the brain, which will in turn potentially provide us with novel insights into healthy and pathological human neuroanatomy. As we have shown in this study, other tissue types than brain can be processed with MASH, opening up the structural investigation of other human organs at the mesoscale. This could make the combination of MASH with ct-dSPIM imaging a powerful tool for anatomical and pathological studies in general. 


\section{References}

1 Duvernoy, H. M., Delon, S. \& Vannson, J. L. Cortical blood vessels of the human brain. Brain Research Bulletin 7, 519-579, doi:https://doi.org/10.1016/0361-9230(81)90007-1 (1981).

2 Rockland, K. S. What do we know about laminar connectivity? Neuroimage 197, 772-784, doi:10.1016/j.neuroimage.2017.07.032 (2019).

3 Rockland, K. S. \& Knutson, T. Axon collaterals of meynert cells diverge over large portions of area V1 in the macaque monkey. Journal of Comparative Neurology 441, 134-147, doi:https://doi.org/10.1002/cne.1402 (2001).

4 Epstein, J. I. et al. The 2014 International Society of Urological Pathology (ISUP) Consensus Conference on Gleason Grading of Prostatic Carcinoma: Definition of Grading Patterns and Proposal for a New Grading System. The American journal of surgical pathology 40, 244-252, doi:10.1097/PAS.0000000000000530 (2016).

$5 \quad$ Nir, G. et al. Automatic grading of prostate cancer in digitized histopathology images: Learning from multiple experts. Medical image analysis 50, 167-180, doi:10.1016/j.media.2018.09.005 (2018).

6 Chung, K. et al. Structural and molecular interrogation of intact biological systems. Nature 497, 332-337, doi:10.1038/nature12107 (2013).

7 Liebmann, T. et al. Three-Dimensional Study of Alzheimer's Disease Hallmarks Using the iDISCO Clearing Method. Cell reports 16, 1138-1152, doi:10.1016/j.celrep.2016.06.060 (2016).

8 Liu, A. K. et al. Bringing CLARITY to the human brain: visualisation of Lewy pathology in three dimensions. Neuropathology and applied neurobiology 42, 573-587, doi:10.1111/nan.12293 (2016).

9 Renier, N. et al. Mapping of Brain Activity by Automated Volume Analysis of Immediate Early Genes. Cell 165, 1789-1802, doi:10.1016/j.cell.2016.05.007 (2016). 
10 Renier, N. et al. iDISCO: a simple, rapid method to immunolabel large tissue samples for volume imaging. Cell 159, 896-910, doi:10.1016/j.cell.2014.10.010 (2014).

11 Sabdyusheva Litschauer, I. et al. 3D histopathology of human tumours by fast clearing and ultramicroscopy. Scientific reports 10, 17619, doi:10.1038/s41598-020-71737-w (2020).

12 Hildebrand, S., Schueth, A., Herrler, A., Galuske, R. \& Roebroeck, A. Scalable Labeling for Cytoarchitectonic Characterisation of Large Optically Cleared Human Neocortex Samples. Scientific reports 9, 10880, doi:10.1038/s41598-019-47336-9 (2019).

13 Zhao, S. et al. Cellular and Molecular Probing of Intact Human Organs. Cell 180, 796-812 e719, doi:10.1016/j.cell.2020.01.030 (2020).

14 Ueda, H. R. et al. Tissue clearing and its applications in neuroscience. Nature reviews. Neuroscience 21, 61-79, doi:10.1038/s41583-019-02501 (2020).

15 Migliori, B. et al. Light sheet theta microscopy for rapid high-resolution imaging of large biological samples. BMC biology 16, 57, doi:10.1186/s12915-018-0521-8 (2018).

16 Pesce, L. et al. Fast volumetric mapping of human brain slices. bioRxiv, 2020.2010.2027.357186, doi:10.1101/2020.10.27.357186 (2020).

17 Sabdyusheva Litschauer, I. et al. 3D histopathology of human tumours by fast clearing and ultramicroscopy. Scientific reports 10, 17619, doi:10.1038/s41598-020-71737-w (2020).

18 Glaser, A. K. et al. Light-sheet microscopy for slide-free non-destructive pathology of large clinical specimens. Nature biomedical engineering 1, doi:10.1038/s41551-017-0084 (2017).

19 Reder, N. P. et al. Open-Top Light-Sheet Microscopy Image Atlas of Prostate Core Needle Biopsies. Archives of pathology \& laboratory medicine 143, 1069-1075, doi:10.5858/arpa.2018-0466-OA (2019).

20 Costantini, I. et al. A versatile clearing agent for multi-modal brain imaging. Scientific reports 5, 9808, doi:10.1038/srep09808 (2015). 
21 Mcllvaine, T. C. A buffer solution for colourimetric comparison. Journal of Biological Chemistry 49, 183-186 (1921).

22 Kumar, A. et al. Dual-view plane illumination microscopy for rapid and spatially isotropic imaging. Nature protocols 9, 2555-2573, doi:10.1038/nprot.2014.172 (2014).

23 Edelstein, A. D. et al. Advanced methods of microscope control using $\mu$ Manager software. Journal of Biological Methods 1, e10, doi:10.14440/jbm.2014.36 (2014).

24 Schindelin, J. et al. Fiji: an open-source platform for biological-image analysis. Nature methods 9, 676-682, doi:10.1038/nmeth.2019 (2012).

25 Horl, D. et al. BigStitcher: reconstructing high-resolution image datasets of cleared and expanded samples. Nature methods 16, 870-874, doi:10.1038/s41592-019-0501-0 (2019).

26 Leuba, G. \& Garey, L. J. Comparison of neuronal and glial numerical density in primary and secondary visual cortex of man. Experimental brain research 77, 31-38, doi:10.1007/BF00250564 (1989).

27 Costantini, I. et al. Large-scale, cell-resolution volumetric mapping allows layer-specific investigation of human brain cytoarchitecture. Biomedical optics express 12, 3684-3699, doi:10.1364/BOE.415555 (2021).

28 Glaser, A. K. et al. Multi-immersion open-top light-sheet microscope for high-throughput imaging of cleared tissues. Nature communications 10, 2781, doi:10.1038/s41467-019-10534-0 (2019). 


\section{Appendix Chapter 5}

Supplementary Video 1. Plane-by-plane view of the resliced MFS 16 scan of occipital lobe slice 1 . The sample is viewed along the $Y Z$ plane, to visualise its full extend. The volume is represented in inverted greyscale.

Supplementary Video 2. Plane-by-plane view of the resliced MFS 16 scan of occipital lobe slice 2 . The sample is viewed along the $Y Z$ plane, to visualise its full extend. The volume is represented in inverted greyscale.

Supplementary Video 3. 3D rendering of an entire human occipital lobe slice 2 ( $3 \mathrm{~mm}$ thick). The slice was imaged with an MFS 16 scan. The mesoscopic and isotropic resolution of the dataset is sufficient to appreciate large anatomical landmarks and differences in the cytoarchitecture.

Supplementary Video 4. 3D rendering of the single view volume obtained the highest resolution possible with the ct-dSPIM. ROls (red spheres) were places manually along the volume, which was rotated and clipped to evaluate the placement of the ROIs within the layers. 
Chapter 5- ct-dSPIM 
CHAPTER 6

\section{hFRUIT: An optimized agent for optical}

\section{clearing of Dil-stained adult human}

\section{brain tissue}

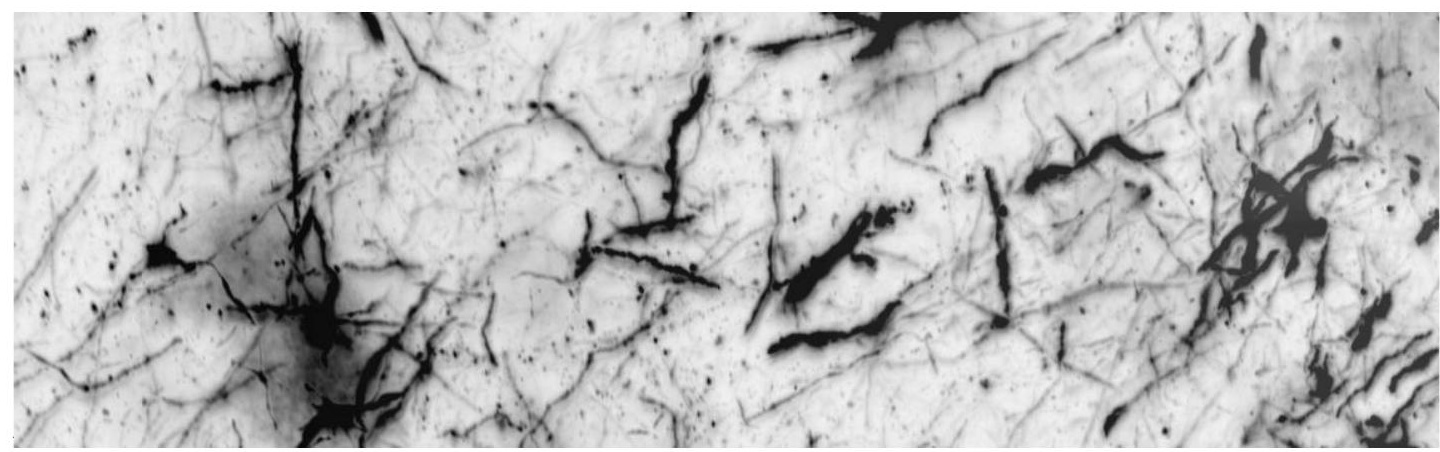

Dil-labelled intrinsic connections in the human amygdala.

Sci Rep 10, 9950 (2020).

https://doi.org/10.1038/s41598-020-66999-3

PMID: 32561795

Sven Hildebrand, Anna Schueth, Klaus von Wangenheim, Christian Mattheyer, Francesco Pampaloni, Hansjürgen Bratzke, Alard Roebroeck \& Ralf Galuske 


\section{Abstract}

Here, we describe a new immersion-based clearing method suitable for optical clearing of thick adult human brain samples while preserving its lipids and lipophilic labels such as 1,1'-dioctadecyl3,3,3',3'-tetramethylindocarbocyanine perchlorate (DiI). This clearing procedure is simple, easy to implement, and allowed for clearing of 5 $\mathrm{mm}$ thick human brain tissue samples within 12 days. Furthermore, we show for the first time the advantageous effect of the PeriodateLysine-Paraformaldehyde (PLP) fixation as compared to the more commonly used $4 \%$ paraformaldehyde (PFA) on clearing performance. 


\section{Introduction}

The rediscovery of optical tissue clearing has attracted a lot of attention in neurobiological research during the last decade. In particular, for studies of the microcircuitry within and between cortical areas, optical clearing in combination with fluorescence microscopy offers new approaches as the very tedious and timeconsuming procedure of axonal reconstruction over large numbers of thin serial sections can be avoided. Numerous clearing protocols have been described and they can be grouped into only a few basic classes of clearing approaches ${ }^{1,2}$. Most of these protocols aim at an everincreasing clearing capacity by removing the lipids from the tissue. To achieve this, they typically use either organic solvents, detergents or both $^{3}$. While such protocols can clear big specimens, they are generally incompatible with lipophilic tracers such as dialkylcarbocyanine and dialkylaminostyryl dyes ${ }^{4}$. However, when it comes to studies on human post mortem tissue probes, lipophilic dyes are currently the only fluorophores allowing investigating microcircuitry at the level of individual neurons and axons which innervate specific regions of interest ${ }^{5}$. While immunofluorescent stainings of e.g., myelin or dendritic fibres allow for comparison on entire fibre populations, insertion of lipophilic dye crystals is currently still the best method available for human tissue, in order to selectively label neuronal processes innervating a specific region of interest. Similarly, there is no immunofluorescent label, which can exclusively highlight neuronal cell bodies in the context of their 
connections to particular regions and layers. The ability of lipophilic dyes for characterizing cortical microcircuitry is therefore unique and a combination of these with optical clearing techniques in human tissue highly desirable. Generally, there are two different approaches to combine lipophilic dyes and optical clearing: The first one involves the use of fixable variants of commonly used lipophilic dyes and an additional fixation after staining which then preserves the staining after delipidation ${ }^{6}$. The second approach aims at clearing tissue samples without delipidation in an aqueous environment. This is a favourable procedure, because labelling with 1,1'-dioctadecyl-3,3,3',3'tetramethylindocarbocyanine perchlorate (DiI) and its derivatives is notoriously difficult in human post mortem material. Even chemically similar dyes like 3,3'-Dioctadecyloxacarbocyanine Perchlorate (DiO) show inferior diffusion properties in post mortem tissue ${ }^{7}$ and it is currently not known, if the fixable versions are suitable for human material at all. Only a few lipid-preserving protocols have been published so far and their clearing capacities are inferior to those of delipidating protocols. Among the first of the lipid preserving clearing protocols were the urea-based Scale $^{8}$ and approaches based on sucrose $^{9}$ or fructose ${ }^{10,11}$. The FRUIT protocol ${ }^{12}$, a combination of Scale ${ }^{8}$ and the fructose-based SeeDB ${ }^{10,11}$, is compatible with lipophilic tracers and exhibits reasonable clearing results in at least mouse and rabbit brains. However, one important disadvantage of SeeDB and FRUIT is the Maillard ${ }^{13}$ reaction between the reducing sugar and tissue proteins, leading to profound tissue browning. Partly due to the Maillard reaction and partly due to the higher lipid content of the 
tissue, the abovementioned protocols deliver poor results in adult human brain tissue and human results have not yet been reported. Newer generations of simple immersion-based clearing protocols use other chemicals with high refractive index (RI) for clearing and are compatible with Dil-staining. However, these protocols are so far either tested in mouse or are limited to depths of about $1 \mathrm{~mm}$ in their clearing capacity in human brain tissue $e^{4,14-17}$.

To overcome all these obstacles, we developed hFRUIT, an optimized version of the original FRUIT protocol, which we tested on thick DiIlabelled tissue samples from different species with high myelin content including human post mortem samples. 


\section{Materials and Methods}

\section{Human tissue}

For DiI-labelling, fresh tissue samples containing the amygdala were dissected after autopsy from the brain of a 39 year old male without any known neurological disorders who had died from a perforating neck wound. The tissue was obtained with a post mortem delay of about $12 \mathrm{~h}$. These samples were not fixed with formalin, but treated as described below (see "Tissue fixation and preparation for DiI application"). Tissue acquisition was approved by the local ethical committee of the Frankfurt University hospital.

Additionally, neocortical tissue samples were taken from human body donors (no known neuropathological diseases) of the body donation program of the Department of Anatomy and Embryology, Maastricht University. These samples were used for macroscopical investigation of the clearing efficacy of hFRUIT in standard formalin fixed brain samples. Donor tissue has also been used for Sudan Black B staining as described below (see "Comparison of lipid content between FRUIT and hFRUIT with Sudan Black B staining"). The tissue donors gave their informed and written consent to the donation of their body for teaching and research purposes as regulated by the Dutch law for the use of human remains for scientific research and education ("Wet op de Lijkbezorging"). Accordingly, a handwritten and signed codicil from the donor posed when still alive and well, is kept at the Department of 
Anatomy and Embryology Faculty of Health, Medicine and Life Sciences, Maastricht University, Maastricht, The Netherlands.

These brains were first fixed in situ by full body perfusion via the femoral artery. Under a pressure of 0.2 bar the body was perfused by 101 fixation fluid (1.8 vol \% formaldehyde, $20 \%$ ethanol, $8.4 \%$ glycerine in water) within 1.5 - 2 hours. Afterwards, the body was preserved for at least 4 weeks for post-fixation submersed in the same fluid. Subsequently, brains were recovered by calvarian dissection and stored in $4 \%$ paraformaldehyde (PFA) in $0.1 \mathrm{M}$ phosphate buffered saline (PBS) for 14 - 30 months.

All methods were carried out in accordance with the relevant guidelines and regulations and all experimental protocols were approved by be Ethics Review Committee Psychology and Neuroscience (ERCPN).

For an overview of the different tissue sources and fixation protocols used in every figure presented, see supplementary table 1.

\section{Porcine tissue}

Our initial experiments were conducted in fresh porcine brain tissue to ensure prudent use of scarce human brain tissue. The fresh tissue was received from a slaughterhouse immediately after sacrificing the animals. Samples were then immersion-fixed as described below. 


\section{Murine tissue}

For comparison of the clearing performance, brains of adult (4 - 5 months) B6.Cg-Tg(Thy1-YFP)HJrs/J mice (Jackson Laboratory) were used. The tissue was provided by the Department for Pharmacology and Toxicology of Maastricht University. The mice were bred under breeding license number B2015-003 and all brains provided were from mice from the breeding colonies, sacrificed as part of the breeding plan (surplus mice from breeding). Animals were sacrificed by $\mathrm{CO}_{2}$ inhalation in accordance with the EU guideline and the local animal regulations. Brains were collected directly and immersion fixated in $4 \%$ PFA in $0.1 \mathrm{M} \mathrm{PBS}$ at $\mathrm{pH} 7.4$ for $24 \mathrm{~h}$ before being transferred to $0.1 \mathrm{M}$ PBS containing $0.1 \%$ sodium azide. Brains were then cut into 3 $\mathrm{mm}$ thick coronal sections for clearing or processed without further dissection.

\section{Tissue fixation and preparation for Dil application}

For Dil-labelling, both fresh porcine and the fresh human amygdala samples which had been dissected after autopsy (see first paragraph of the method section), were immediately fixed in Periodate-LysineParaformaldehyde (PLP) fixative ${ }^{18}$. This fixative substantially facilitates the diffusion of DiI and is considered to be important for a highquality DiI-labelling in human post mortem tissue, as first shown by Burkhalter and Bernado ${ }^{19,20}$. The fixative consists of 0.1M PBS at pH 7.4, $2.6 \%$ PFA, $0.8 \%$ iodoacetic acid, $0.8 \%$ sodium periodate, and $0.1 \mathrm{M}$ D-L-lysine and the samples were fixed prior to labelling for 2-7 days. 
After labelling, samples were kept in $2 \%$ PFA in $0.1 \mathrm{M} \mathrm{PBS}$ at pH 7.4 for 3 months (porcine samples) or at least 6 months up to several years (human samples) at $37^{\circ} \mathrm{C}$ in the dark, with the fixative being replaced regularly.

\section{Post mortem axonal tracing with Dil}

After the initial PLP fixation for 2-7 days described above, samples were washed in 0.1 M PBS. For Dil labelling, a small incision was made in the grey matter of the tissue blocks and crystals of the dye (Thermo Fisher Scientific Inc., Waltham, Massachusetts, USA) were placed into the tissue using an ethanol filled glass pipette with a tip diameter of approx. $50 \mu \mathrm{m}$. Special care was taken to avoid direct contact of the dye crystals with the white matter.

\section{Optimisation of hFRUIT clearing for human brain tissue}

In our new clearing protocol, we increased 1-thioglycerol (M1753, Sigma-Aldrich Corp., St. Louis, Missouri, USA) concentrations to minimize the Maillard-reaction caused by high fructose concentrations. Moreover, 1-thioglycerol has a high refractive index (RI) and, therefore, a positive effect on the clearing performance. In contrast to previous protocols, which exclusively used fructose, the fructose concentration was reduced in hFRUIT and compensated with the non-reducing sugar sucrose (Merck KGaA, Darmstadt, Germany). This further minimizes the Maillard-reaction. In order to optimize the composition of the different components of the clearing solution, the 
content of the different components was systematically varied (see table 1). All clearing steps were carried out at room temperature on a shaker. For an accelerated dissolution of the $80 \%$ and $100 \%$ solutions, fructose, sucrose, and 1-thioglycerol should be mixed under stirring in a small volume of distilled water at $60-70{ }^{\circ} \mathrm{C}$ until completely dissolved. The solution must cool down before adding urea to avoid its decomposition and distilled water is added up to the final volume for the respective concentration. All samples were incubated in $50 \mathrm{ml}$ tubes filled with the solution. Before the immersion in the final 100\% hFRUIT solution, samples were carefully blotted on paper tissues and new $50 \mathrm{ml}$ tubes were used, to avoid contamination of the lower concentrated solution. The final RI of the hFRUIT solution was between $1.49-1.50$ and thus very close to that of immersion oil (1.52).

\section{FRUIT clearing of the brain tissue}

At first, FRUIT clearing was performed according to the original publication $^{12}$. Samples were incubated in ascending concentrations of FRUIT solution containing $20 \%, 40 \%, 60 \%, 80 \%$, and $100 \%$ (w/v) fructose respectively. All solutions contained $0.5 \%(\mathrm{w} / \mathrm{v})$ 1thioglycerol and $24 \%(\mathrm{w} / \mathrm{v})$ urea and were dissolved in distilled water. Incubation was carried out for $8 \mathrm{~h}$ (20-60\%), 12h (80 \%), and 24h $(100 \%)$ at $37^{\circ} \mathrm{C}$. Because of the intensive tissue-browning due to the Maillard reaction observed at these elevated temperatures, FRUIT was rather carried out at room temperature (RT). For better comparison with the hFRUIT protocol, the same incubation times were used on 
both murine and porcine brain samples cleared with either FRUIT or hFRUIT as described above: 1 day in $20 \%, 2$ days each in $40 \%$ and $60 \%$, and 3 days each in $80 \%$ and $100 \%$ (see also table 1). All samples were incubated on a shaker in $50 \mathrm{ml}$ tubes filled with the solution.

Comparison of lipid content between FRUIT and hFRUIT with Sudan Black B staining

Human neocortex samples obtained from body donors, which have been perfusion fixed in formalin and post-fixed in 4\% PFA, were taken from the same gyrus and either treated with hFRUIT as described above or stored in $0.1 \mathrm{M}$ PBS with $1 \%$ sodium azide as a control. After clearing, the samples were immersed again in 0.1 M PBS and cut into $50 \mu \mathrm{m}$ sections on a vibratome (VT1200 S, Leica Biosystems GmbH, Wetzlar, Germany). Sections were mounted on gelatinized glass slides and air dried overnight. Then, sections were washed in distilled water for $5 \mathrm{~min}$, dehydrated in $70 \%$ ethanol for $5 \mathrm{~min}$ and stained in $0.1 \%$ Sudan Black B (Merck KGaA, Darmstadt, Germany) in 70 \% ethanol for 10 min. Excess dye was washed out in $70 \%$ ethanol and distilled water for 5 min each before cover-slipping in Kaiser's glycerol gelatine (Carl Roth GmbH \& Co. KG, Karlsruhe, Germany).

\section{Confocal laser scanning microscopy of porcine brain samples}

Samples were imaged on a LSM 780 Axio Observer (Carl Zeiss AG, Jena, Germany) equipped with a Plan-Apochromat 10x/0.3 M27 objective. For imaging of autofluorescent vasculature, $488 \mathrm{~nm}$ excitation and a 
detection wavelength between 493 and $552 \mathrm{~nm}$ was chosen. For imaging of the Dil signal, $514 \mathrm{~nm}$ was used for excitation and a detection band of 546-672 $\mathrm{nm}$.

\section{Light-sheet microscopic imaging of human brain samples}

Before imaging, samples were taken out of the 100\% hFRUIT solution and carefully blotted dry with paper towels. After that the samples were immersed in a 1:1 mixture of mineral oil (M8410, Sigma-Aldrich Corp., St. Louis, Missouri, USA) and silicone oil (175633, Sigma-Aldrich Corp., St. Louis, Missouri, USA). This solution has the same RI as the clearing solution but is much less viscous and allows easier handling. Imaging was performed on a diSPIM set-up (Applied Scientific Instrumentation Inc., Eugene, Oregon, USA) with objectives tuneable to RIs of 1.33-1.56, resulting in a numerical aperture (NA) of 0.37-0.43 and $15.3 \mathrm{x}-17.9 \mathrm{x}$ magnification respectively (Special Optics Inc., Denville, New Jersey, USA). For excitation, a $561 \mathrm{~nm}$ laser-line was used.

\section{Comparison of imaging depth}

For the comparison of the clearing capacity of FRUIT and hFRUIT, 6 porcine brain samples for each clearing approach were imaged on a confocal microscope as described above and z-stacks with a step size of $35 \mu \mathrm{m}$ of the autofluorescent signal of blood vessels in 3 regions of interest (ROIs) per sample were acquired. Subsequently, the clearing capacity of each protocol was quantified on the unprocessed image 
files. To this end, the maximally achievable imaging depth was assessed by the contrast decay of the median pixel intensity value per plane in the acquired stacks for both clearing protocols as has been described earlier by Costantini et al. ${ }^{21}$. The distribution of measured imaging depths of FRUIT and hFRUIT cleared samples was tested for normal distribution with a Kolmogorov-Smirnov test as well as an Anderson-Darling test. For comparison between FRUIT and hFRUIT cleared samples, an unpaired t-test was used.

\section{Data processing}

The data sets acquired with the light-sheet microscope were processed with Huygens Professional (v19.10; Scientific Volume Imaging B.V., Hilversum, the Netherlands). The raw data was first deskewed using the light-sheet deskewing mode in the Huygens Object Stabilizer. The point spread function was extracted from the deskewed dataset using the PSF distiller and the data was subsequently deconvolved with the extracted PSF using the Huygens CMLE algorithm (40 iterations, SNR 40, manual background). Maximum intensity projections or 3D renderings were also generated with Huygens Professional. For all other processing steps of the images such as brightness and contrast adjustments, or denoising the open-source platform FIJI was used ${ }^{22}$. For the assessment of imaging depth as described above, a custom-made script in MATLAB R2015a (The MathWorks, Inc., Natick, Massachusetts, USA) was used. Statistical analysis was likewise performed in MATLAB. 


\section{Results}

\section{Optimisation of the hFRUIT protocol}

The optimal parameters for the hFRUIT protocol are given in table 1. Adding the non-reducing sugar sucrose to the solution increased the clearing capacity. Surprisingly, exchanging fructose entirely with sucrose did not produce markedly better clearing results as compared to the sucrose/fructose mixture, even though the Maillard reaction should have been minimized. This is probably due to the fact that in the mixture in total a higher amount of sugar can be dissolved, which further increases the RI. Nevertheless, the solution containing only sucrose as sugar displayed less tissue browning and improved transparency as compared to the original fructose based FRUIT protocol.

The biggest effect on the enhancement of clearing performance, however, was the increase of the 1-thioglycerol concentration from $0.5 \%(\mathrm{w} / \mathrm{v})$ as in the original protocol to $20 \%(\mathrm{w} / \mathrm{v})$ as a final concentration. This concentration was the highest possible for $\alpha-$ thioglycerol, because the dissolution of urea and sugars is no longer possible above the concentration of $20 \%(\mathrm{w} / \mathrm{v})$. 
Table 1: Concentrations and incubation times for the hFRUIT clearing protocol. The times indicated here have been used for all the samples used in this study. However, incubation times depend on samples size and have to be determined empirically and smaller samples are expected to clear faster.

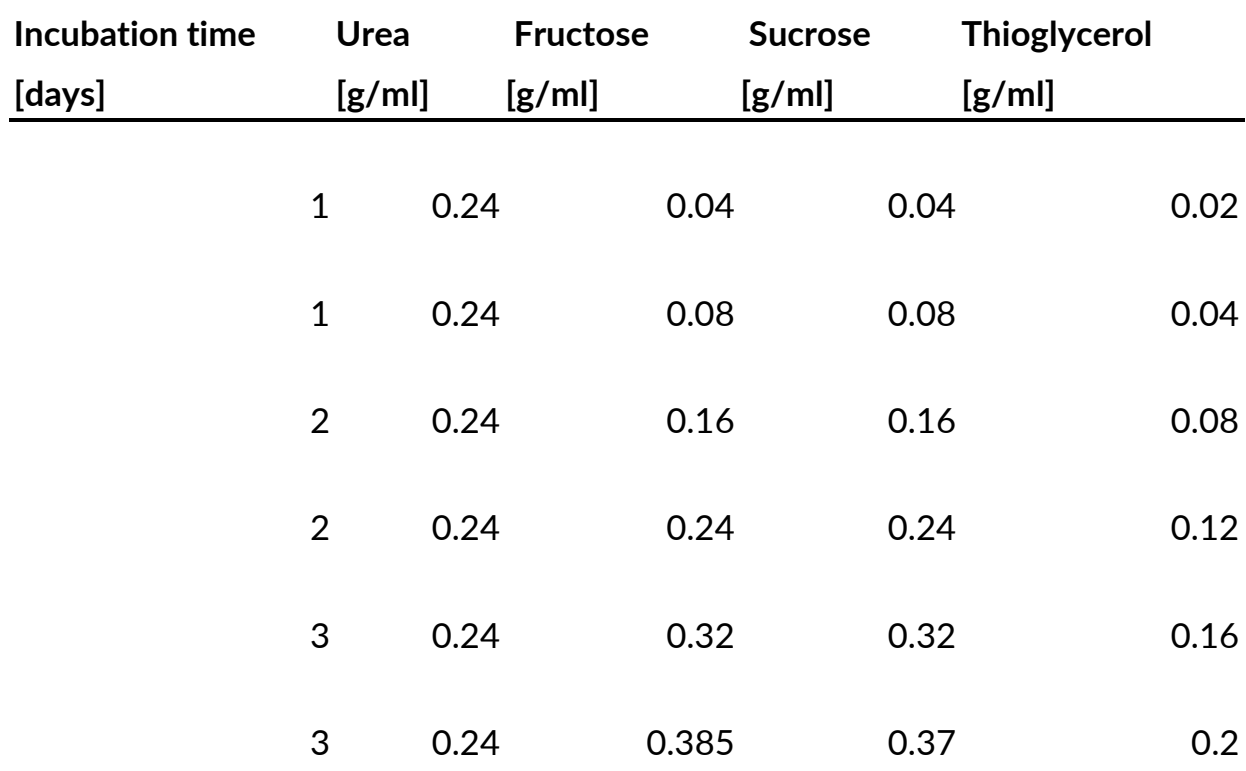

\section{Comparison of FRUIT and hFRUIT in murine and porcine brain tissue samples}

In order to compare the clearing capacity of our new solutions both, $3 \mathrm{~mm}$ thick coronal sections and entire mouse brains were cleared. Upon macroscopic inspection, samples treated with hFRUIT appeared much more transparent as compared to the original protocol (Fig. 1 a and b, Supplementary Fig. 1). This was mainly due to reduced tissue browning. 

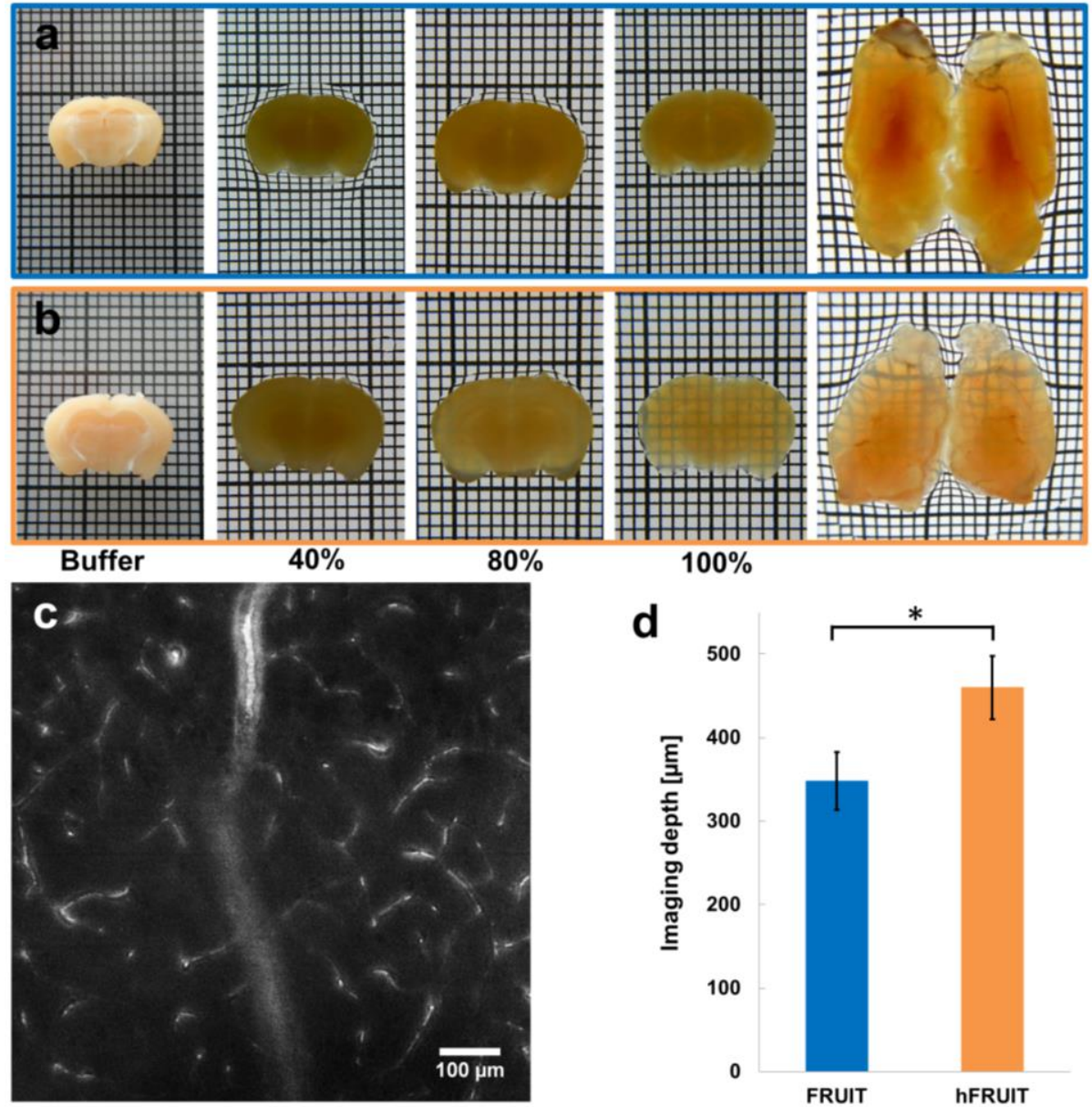

Figure 1: Comparison of the clearing capacity of FRUIT and hFRUIT on nonhuman brain samples. Comparison of murine brain samples cleared with FRUIT (a; blue) and hFRUIT (b; orange). The first four panels in $\mathbf{a}$ and $\mathbf{b}$ show $3 \mathrm{~mm}$ thick coronal brain sections at varying points during the clearing process. The last panels in $\mathbf{a}$ and $\mathbf{b}$ show two whole hemispheres after clearing. The heavily myelinated regions in the medial and posterior parts of the brain are generally more difficult to clear without delipidation. (grid: $1 \times 1 \mathrm{~mm}$ ). (c) typical example of autofluorescent background with $488 \mathrm{~nm}$ excitation in a $3 \mathrm{~mm}$ thick porcine brain sample cleared with hFRUIT at a depth of $315 \mu \mathrm{m}$ at 10x magnification. Legend continued on the next page. 
Figure 1: Continued. (d) contrast decay of autofluorescent signal as function of depth (Costatini et al., 2015) ${ }^{21}$ in porcine samples cleared with FRUIT (blue) or hFRUIT (orange; $\mathrm{n}=18$ respectively; error bars indicate SEM; unpaired $\mathrm{t}$-test: $p=0,0361)$.

In order to quantify this difference and to test the clearing capacity on larger and more myelinated tissue, $3 \mathrm{~mm}$ thick porcine brain sections were cleared with either method and the autofluorescent signal of cortical vasculature was imaged (Fig. 1 c). The maximally achievable imaging depth of both methods was assessed by comparing the contrast decay of the median pixel intensity value per plane in the acquired stacks as has been described earlier by Costatini et al. ${ }^{21}$. The average depth to which samples could be imaged was found to be greater in tissue cleared with hFRUIT (unpaired t-test: $\mathrm{p}=0.0361$ ), despite the relatively short excitation wavelength of $488 \mathrm{~nm}$, confirming our observations in the murine samples (Fig. $1 \mathrm{~d}$ ).

\section{Preservation of Dil label after clearing}

Before applying the hFRUIT protocol to human brain tissue samples, we tested whether the DiI label is preserved after our clearing procedure. To this end $3 \mathrm{~mm}$ thick porcine brain samples were incubated with DiI crystals for 3 months and then cleared and imaged using an inverted confocal microscope. As shown in figure 2 and supplementary figure 2 , the label was well preserved after clearing and the longer excitation wavelength allowed for even deeper imaging as 
compared to the wavelength used for the autofluorescence imaging. Individual cell bodies and fibres were clearly discernible at depths up to $1.5 \mathrm{~mm}$ and more.
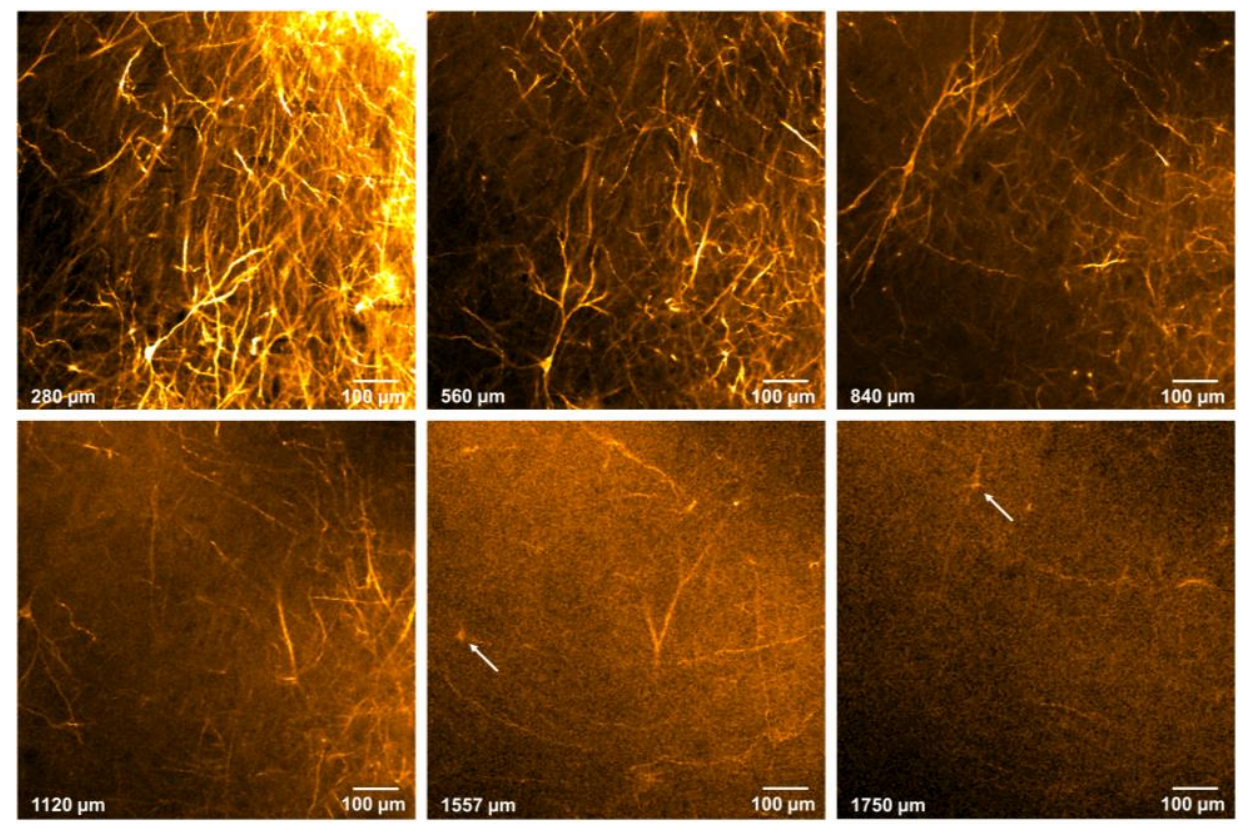

Figure 2: Dil stained neurons and neuronal processes in a porcine brain sample cleared with hFRUIT. Panels show different planes from the same z-stack imaged with CLSM (10x magnification). Despite the increasing background over depth, cell bodies and processes are visible up to at least $1500 \mu \mathrm{m}$ (white arrows).

\section{Capacity of hFRUIT for clearing human brain tissue}

First, the clearing capacity of hFRUIT was tested on unlabelled human neocortical samples of $5 \mathrm{~mm}$ thickness fixated with formalin. As expected, the clearing in grey matter was better as compared to white 
matter, as the difference in RI between white and grey matter cannot be alleviated without delipidation (Fig. 3 a). When the same clearing method was used on DiI-labelled amygdala samples treated with the PLP fixation, the transparency was superior for grey matter regions (Fig. 3 b).

a

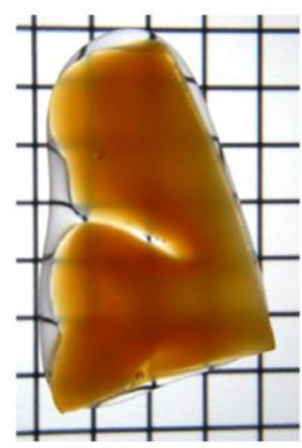

b
Formalin fixed

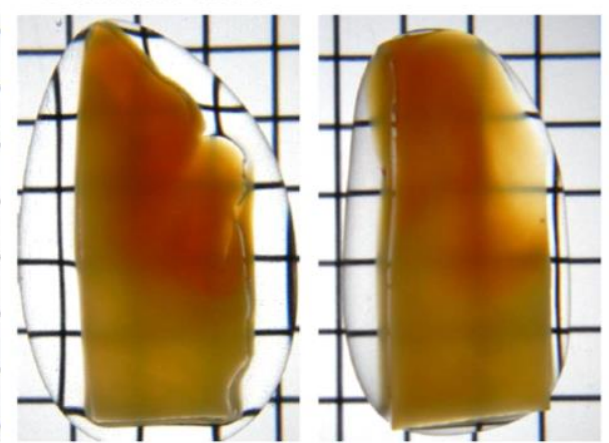

PLP fixed

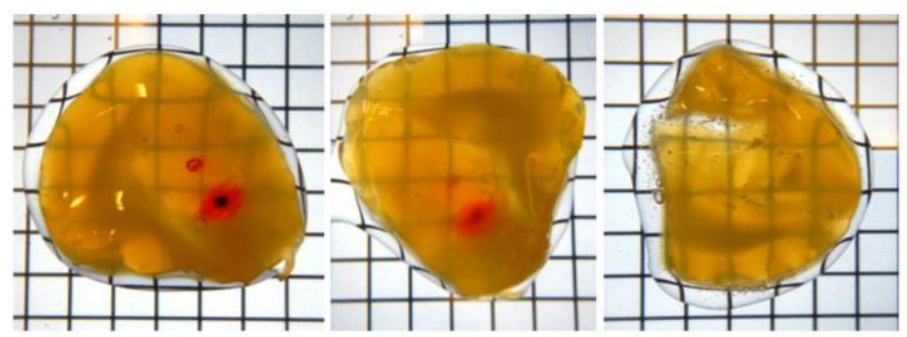

Figure 3: Effect of fixative on clearing capacity with hFRUIT. Transparency of $5 \mathrm{~mm}$ thick human neocortex samples fixed with formalin is limited (a). When samples are fixed with Burkhalter and Bernado's variation of the PLP-fixation (b; Burkhalter and Bernado, 1989) the transparency is considerably improved, as shown here on 5-7 mm thick human amygdala samples. Note that both injection sites are clearly visible from the backside through the entire thickness of the sample in the left and middle panel. Legend continued on the next page. 
Figure 3: Continued. In this sample the thickness varies from about $7 \mathrm{~mm}$ (on the left side in left panel) and $5 \mathrm{~mm}$ on the opposite side. The second sample has a more even thickness of about $5 \mathrm{~mm}$. Grid: $5 \times 5 \mathrm{~mm}$.

The first sample was wedge shaped and approximately $7 \mathrm{~mm}$ thick on one side (left side of the tissue in the first panel of Fig. $3 \mathrm{~b}$ ), and about $5 \mathrm{~mm}$ thick on the other side (right side in Fig. $3 \mathrm{~b}$ first panel). The second sample was more evenly cut at about $5 \mathrm{~mm}$ thickness and appears a bit lighter overall because of this. However, even on thinner tissue regions, the heavily myelinated white matter rendered parts containing a lot of fibre bundles more opaque, as the abundance of lipids changes the RI in the respective tissue regions.

The human amygdala samples were imaged on a diSPIM system (Fig. 4, Supplementary Video 1-2). Images were acquired in the vicinity of the injection site of the DiI crystal (bright region in upper part of Fig. $4 \mathrm{a}$ and $\mathrm{c}$ ). The preservation of the label in human tissue was excellent and provided a strong contrast of individual fibres to the background (Fig. 4 b). Whole cells could be observed, including their dendrites with protrusions which might be indicative of spines (Fig. $4 \mathrm{c}$ and d). 

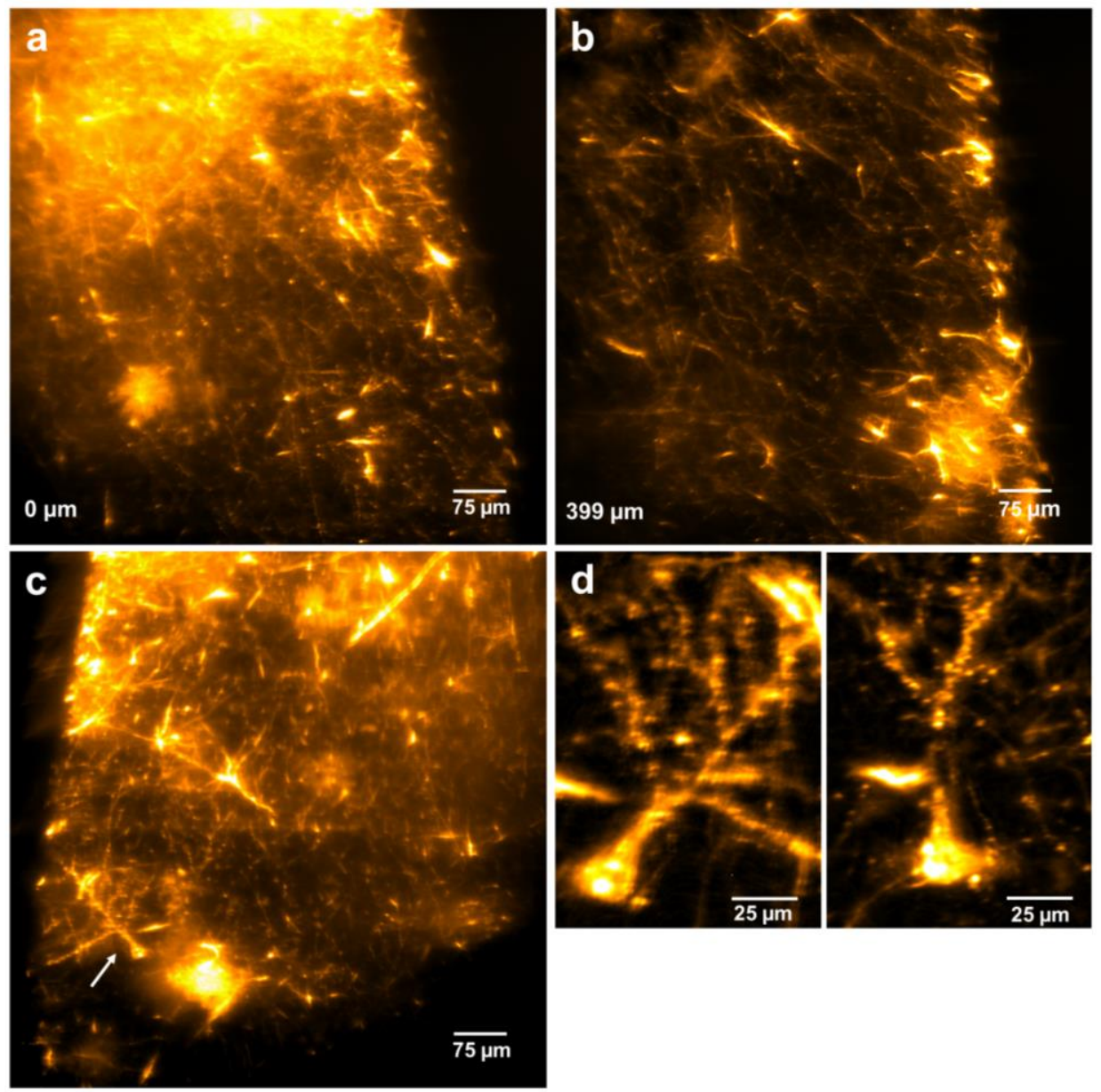

Figure 4: Light-sheet microscopy of Dil-stained human amygdala. (a) The Dil label in close proximity to the injection site of the crystal (upper part of the image). (b) Label of individual fibres $400 \mu \mathrm{m}$ lateral from the injection site was well preserved. (c) The staining quality after clearing was good enough to clearly distinguish labelled neurons (arrow). (d) Enlarged maximum intensity projections over $10 \mu \mathrm{m}$ of two labelled neurons with dendrites including spinelike protrusions clearly visible. 
The volume was acquired as an $\mathrm{x}$-scan over a region of $400 \times 772 \times 546 \mu \mathrm{m}$ starting in close proximity to the injection site. A single view dataset was taken for further processing with the Huygens software for deconvolution.

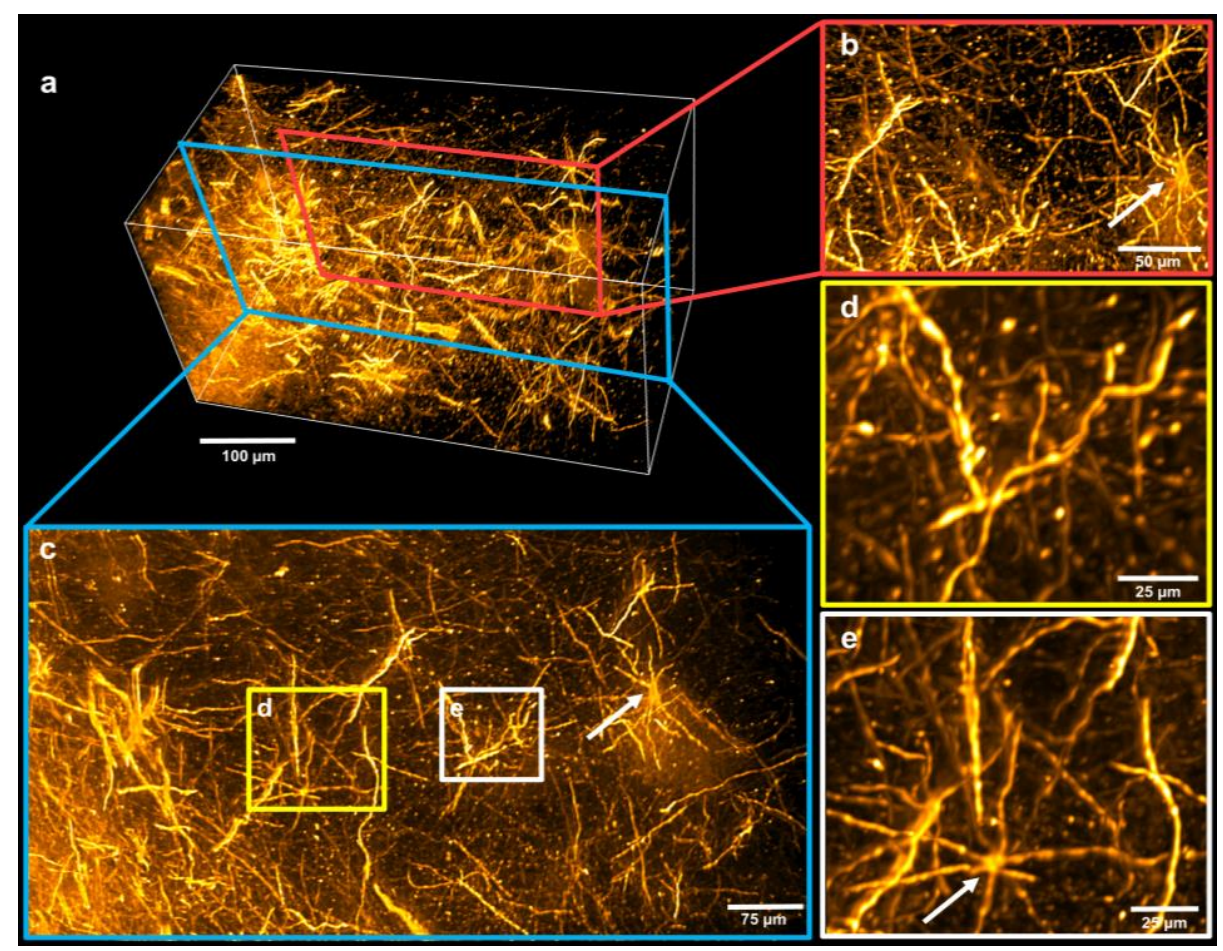

Figure 5: Deconvolved light-sheet data. (a) 3D rendering of a sub-volume of one view. (b) Magnified view of a part of the rendering showing cell bodies (arrow) and fibres throughout the field of view. (c) Maximum intensity projection over $60 \mu \mathrm{m}$ of the volume. (d) and (e) Enlarged regions of the projection showing neuron soma (arrow) and good contrast of even the smallest stained nerve fibres. 
After this steps, individual fibres could be easily identified in 3D renderings of a sub-volume from all sides (Fig. 5a and b, Supplementary Video 3) and stood out clearly in maximum intensity projections over a depth of $60 \mu \mathrm{m}$ (Fig. $5 \mathrm{c}-\mathrm{e}$ ).

In order to access the lipid-preserving properties of our clearing technique, additional hFRUIT treated samples were rehydrated and 50 $\mu \mathrm{m}$ thick sections were cut with a vibratome. These sections were stained with Sudan Black B as a general lipid marker and compared to uncleared controls. Both groups were intensely stained both in white and grey matter (Fig. 6). As seen already with DiI, individual fibres in the grey matter remained well discernible within the light blue neuropil (white arrows in Fig. $6 \mathrm{~b}$ and $\mathrm{d}$ and insets therein). The intensely stained spots visible throughout the grey matter of both groups are likely to be clusters of lipofuscin, a lipid containing pigment that accumulates over age as a product of lipid metabolism23,24. These findings demonstrate that our new clearing protocol leaves a substantial part of the brain lipid fraction intact and, thus, accessible for tracing techniques using lipophilic dyes. At the same time our new protocol is able to render highly myelinated tissues transparent over several millimetres, which makes it a highly promising approach for tracer studies in cleared adult human brain tissue. 

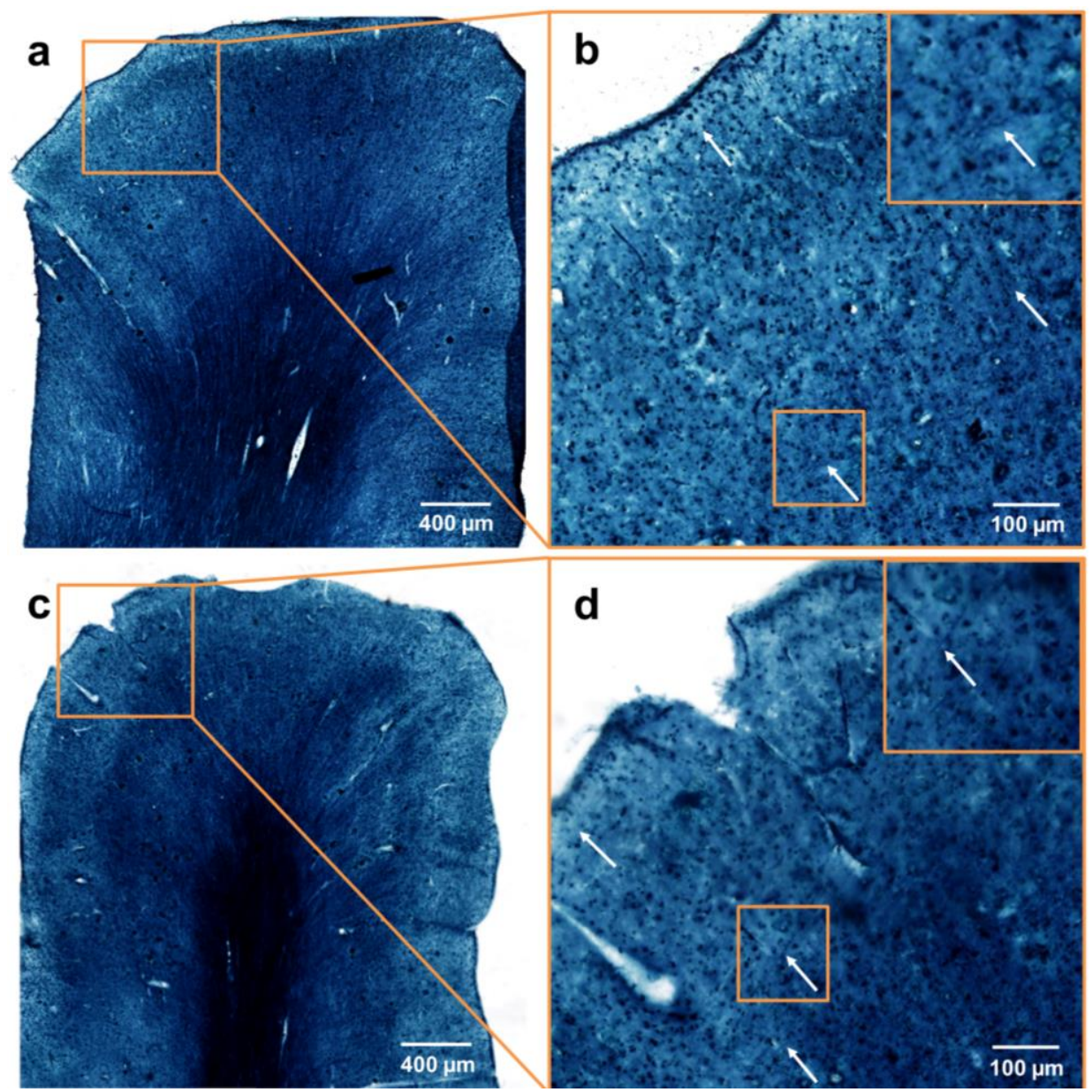

Figure 6: Sudan Black B staining for lipids on uncleared human brain sections ( $a$ and b) and sections of an hFRUIT cleared sample (c and d). For both conditions the abundance of lipids is evident by the dark blue-black staining. At lower magnification, the intensely stained white matter as well as radial fibre bundles are clearly distinguishable over a general light blue background ( $a$ and c). Likewise, in higher magnifications of ROIs in superficial cortical regions, individual tangential fibers can be detected in both cleared (b) and uncleared (d) sections (arrows in $\mathbf{b}$ and $\mathbf{d}$ and insets therein from indicated regions). The intensely stained small spots visible in the grey matter of both conditions are likely lipofuscin aggregates. 


\section{Discussion}

The hFRUIT protocol allows for the first time clearing of fixed adult human brain tissue of several millimetre thickness while leaving the majority of lipids intact. The original FRUIT protocol already provided reasonable performance in murine and rabbit brain tissue, but the clearing capacity in higher mammals and especially human tissue was very limited with this type of clearing approaches ${ }^{11,12,15,25,26}$. Clearly, the higher myelin content of human brain tissue is the main reason for this. Even though delipidating clearing approaches might lead to better results in human brain tissue, such approaches cannot be used in circumstances which require preservation of lipids. One of the most important instances in this context is the targeted study of microcircuits. So far the use of lipophilic tracers such as DiI is the only approach to study microcircuitry in a targeted manner in the human brain. Even though fixable lipophilic dyes are available6, it is currently unknown whether these dyes have the same staining capacity as the traditional dyes such as DiI and DiA. The labelling procedure in human post mortem material with DiI crystals is notoriously difficult and modified fixable derivatives might not have the same diffusion properties in human tissue as compared to other mammalian brains. In our experience the best staining results depend on the following factors: a post mortem interval of less than 24 hours $^{27-30}$, PLP fixation of the tissue ${ }^{19,20,30}$, and incubation at $37^{\circ} \mathrm{C}$ in the dark to enhance diffusion. 
Therefore, hFRUIT offers in principle a great advantage when aiming at tracing local circuits in the human brain on the level of individual axons. However, currently some aberrations can be observed in our data set which might limit the ability of accurate tracing. The aberrations occur mainly in the periphery of the image while in the centre, individual fibres are clearly visible (see Fig. 4, Supplementary Video 1-2). Thus, it is unlikely that these aberrations result from RI inhomogeneities between the tissue and the imaging solution, because these aberrations should be equally distributed throughout the imaging plane. It is more likely that aberrations result from small discrepancies in the calibration of the microscope set-up as the objectives used for imaging were prototypes (kindly provided for testing by Applied Scientific Instrumentation Inc., Eugene, Oregon, USA) and tested for the first time on tissue samples.

While the magnification of these objectives is enough to demonstrate single fibres, the clear visualisation of dendritic spines is currently not unequivocally possible. Even though the protrusions observed on dendrites (Fig. 4d) might be indicative of spines, it cannot be excluded that they are results of blebbing due to apoptosis or tissue degradation in the post mortem brains before fixation. Future studies using higher magnification objectives and multi-view deconvolution, will allow for complete 3D reconstruction over large volumes of the dendritic arbors of DiI-labelled neurons. This might enable circuitry visualisation at a quality level which is usually achieved using Golgi 
stains or intracellular fillings in thin sections of human post mortem brain tissue ${ }^{5}$.

Much to our surprise, modification of the fixation protocol from a simple formalin fixation to the PLP fixation greatly enhanced the efficacy of hFRUIT (Fig. 3). Even though the PLP fixation had been chosen, because it improves the Dil staining, it also improved the transparency obtained with hFRUIT. The reason for the enhanced clearing of samples fixed with the PLP fixation is currently unknown. However, it is unlikely that only the lower concentration of PFA is responsible for this finding. The long incubation times in fixative of at least three months should be sufficient to allow for complete fixation of the tissue, despite the lower PFA concentrations of $2.6 \%$ in the initial and $2 \%$ in the post-fixation ${ }^{7}$. Therefore, the beneficial effect must be due to either the different chemical composition of the tissue by preservation of other tissue components or by interactions between the compounds of the fixative and the clearing solution. We consider this observation to be of high interest. Future investigations should focus on testing alternative fixatives, such as the recently proposed glyoxal fixation ${ }^{31}$ or the PLP fixation used in this study in order to establish new standard fixatives for clearing and expansion microscopy ${ }^{32-34}$. Since these alternative solutions supposedly preserve the ultrastructure of the tissue much better than formalin or $4 \%$ PFA while retaining compatibility with e.g. immunohistochemistry they could be of particular interest for super-resolution microscopy on expanded samples ${ }^{18,31,35}$. 
For future investigations using the hFRUIT approach, a simple counterstaining procedure is desirable to provide the relevant cytoarchitectural framework for the interpretation of the connections visualised with DiI. The recently developed MASH approach ${ }^{36}$ provides options for a suitable counterstaining, if the staining method is combined with hFRUIT clearing. Likewise, the reconstruction of the local circuitry revealed with hFRUIT and the tracing of these connections in 3D will be the focus of future investigations.

The combination with the above mentioned improvements will help to advance the understanding of the intrinsic cortical circuitry in humans, where the available range of techniques is considerably more limited as compared to animal models. 


\section{Conclusions}

We successfully developed hFRUIT, an improved immersion-based protocol capable of clearing human brain tissue while preserving its lipids and lipophilic dyes such as DiI. This makes it a great tool to easily study brain microcircuits in large human tissue blocks in three dimensions. The protocol is simple and achieved clearing of $5 \mathrm{~mm}$ thick human brain samples in 12 days. Furthermore, it is important to note that different fixatives crucially influence the clearing performance as well. This is of great interest to the clearing community as it might allow for improving the performance of other clearing protocols. This holds not only for human tissue, but is also important for animal models especially as the choice of fixative may be more flexible as compared to human post mortem tissue. 


\section{References}

1. Richardson, D. S. \& Lichtman, J. W. SnapShot: Tissue Clearing. Cell 171, 496-496.e1 (2017).

2. Richardson, D. S. \& Lichtman, J. W. Clarifying Tissue Clearing. Cell 162, 246-257 (2015).

3. Tainaka, K., Kuno, A., Kubota, S. I., Murakami, T. \& Ueda, H. R. Chemical Principles in Tissue Clearing and Staining Protocols for Whole-Body Cell Profiling. Annu. Rev. Cell Dev. Biol. 32, 713-741 (2016).

4. Chen, L. et al. UbasM: An effective balanced optical clearing method for intact biomedical imaging. Sci. Rep. 7, 1-11 (2017).

5. Lanciego, J. L. \& Wouterlood, F. G. A half century of experimental neuroanatomical tracing. Journal of Chemical Neuroanatomy 42, 157183 (2011).

6. Jensen, K. H. R. \& Berg, R. W. CLARITY-compatible lipophilic dyes for electrode marking and neuronal tracing. Sci. Rep. 6, 32674 (2016).

7. Chen, B. K. et al. Optimizing conditions and avoiding pitfalls for prolonged axonal tracing with carbocyanine dyes in fixed rat spinal cords. J. Neurosci. Methods 154, 256-263 (2006).

8. Hama, H. et al. Scale: A chemical approach for fluorescence imaging and reconstruction of transparent mouse brain. Nat. Neurosci. 14, 14811488 (2011).

9. Tsai, P. S. et al. Correlations of Neuronal and Microvascular Densities in Murine Cortex Revealed by Direct Counting and Colocalization of Nuclei and Vessels. J. Neurosci. 29, 14553-14570 (2009).

10. Ke, M. T., Fujimoto, S. \& Imai, T. SeeDB: A simple and morphologypreserving optical clearing agent for neuronal circuit reconstruction. Nat. Neurosci. 16, 1154-1161 (2013). 
11. Ke, M. T. \& Imai, T. Optical clearing of fixed brain samples using SeeDB. Curr. Protoc. Neurosci. (2014). doi:10.1002/0471142301.ns0222s66

12. Hou, B. et al. Scalable and Dil-compatible optical clearance of the mammalian brain. Front. Neuroanat. 9, 1-11 (2015).

13. Dills W L, J. Protein fructosylation: fructose and the Maillard reaction. Am. J. Clin. Nutr. 58, 779S-787S (1993).

14. Aoyagi, Y., Kawakami, R., Osanai, H., Hibi, T. \& Nemoto, T. A rapid optical clearing protocol using 2,2' -thiodiethanol for microscopic observation of fixed mouse brain. PLoS One 10, 629-630 (2015).

15. Li, W., Germain, R. N. \& Gerner, M. Y. Multiplex, quantitative cellular analysis in large tissue volumes with clearing-enhanced 3D microscopy (C e 3D). Proc. Natl. Acad. Sci. 201708981 (2017). doi:10.1073/pnas.1708981114

16. Lai, H. M., Ng, W. L., Gentleman, S. M. \& Wu, W. Chemical Probes for Visualizing Intact Animal and Human Brain Tissue. Cell Chem. Biol. 24, 659-672 (2017).

17. $\mathrm{Yu}, \mathrm{T}$. et al. RTF: A rapid and versatile tissue optical clearing method. Sci. Rep. 8, 1-9 (2018).

18. McLean, I. W. \& Nakane, P. K. Periodate lysine paraformaldehyde fixative. A new fixative for immunoelectron microscopy. J. Histochem. Cytochem. 22, 1077-1083 (1974).

19. Burkhalter, A. \& Bernardo, K. L. Organisation of corticocortical connections in human visual cortex. Proc. Natl. Acad. Sci. 86, 1071-1075 (1989).

20. Burkhalter, A., Bernardo, K. L. \& Charles, V. Development of local circuits in human visual cortex. J. Neurosci. 13, 1916 LP - 1931 (1993).

21. Costantini, I. et al. A versatile clearing agent for multi-modal brain imaging. Sci. Rep. 5, 9808 (2015). 
22. Schindelin, J. et al. Fiji: An open-source platform for biological-image analysis. Nat. Methods 9, 676-682 (2012).

23. Schnell, S. A., Staines, W. A. \& Wessendorf, M. W. Reduction of lipofuscinlike autofluorescence in fluorescently labeled tissue. J. Histochem. Cytochem. 47, 719-730 (1999).

24. Gray, D. A. \& Woulfe, J. Lipofuscin and aging: a matter of toxic waste. Sci. Aging Knowledge Environ. 2005, re1-re1 (2005).

25. Ke, M. T. et al. Super-Resolution Mapping of Neuronal Circuitry With an Index-Optimized Clearing Agent. Cell Rep. 14, 2718-2732 (2016).

26. Lai, H. M. et al. Author Correction: Next generation histology methods for three-dimensional imaging of fresh and archival human brain tissues (Nature Communications (2018) 9 (1066) DOI: 10.1038/s41467-01803359-w). Nat. Commun. 9, (2018).

27. Ferrer, I., Martinez, A., Boluda, S., Parchi, P. \& Barrachina, M. Brain banks: Benefits, limitations and cautions concerning the use of post-mortem brain tissue for molecular studies. Cell Tissue Bank. 9, 181-194 (2008).

28. Ferrer, I. et al. Brain protein preservation largely depends on the postmortem storage temperature: Implications for study of proteins in human neurologic diseases and management of brain banks: A BrainNet Europe study. J. Neuropathol. Exp. Neurol. 66, 35-46 (2007).

29. Crecelius, A. et al. Assessing quantitative post-mortem changes in the gray matter of the human frontal cortex proteome by 2-D DIGE. Proteomics 8, 1276-1291 (2008).

30. Galuske, R. A. W., Schlote, W., Bratzke, H. \& Singer, W. Interhemispheric asymmetries of the modular structure in human temporal cortex. Science (80-. ). 289, 1946-1949 (2000).

31. Richter, K. N. et al. Glyoxal as an alternative fixative to formaldehyde in immunostaining and super-resolution microscopy. EMBO J. 37, e201695709 (2017). 
32. Chen, F., Tillberg, P. W. \& Boyden, E. S. Expansion microscopy. Science (80-. ). 347, 543 LP - 548 (2015).

33. Murakami, T. C. et al. A three-dimensional single-cell-resolution wholebrain atlas using CUBIC-X expansion microscopy and tissue clearing. Nat. Neurosci. 21, 625-637 (2018).

34. Tillberg, P. W. et al. Protein-retention expansion microscopy of cells and tissues labeled using standard fluorescent proteins and antibodies. Nat. Biotechnol. 34, 987-992 (2016).

35. HALL, P. A., STEARN, P. M., BUTLER, M. G. \& D'ARDENNE, A. J. Acetone/periodate-lysine-paraformaldehyde (PLP) fixation and improved morphology of cryostat sections for immunohistochemistry. Histopathology 11, (1987).

36. Hildebrand, S., Schueth, A., Herrler, A., Galuske, R. \& Roebroeck, A. Scalable cytoarchitectonic characterisation of large intact human neocortex samples. bioRxiv 274985 (2018). doi:10.1101/274985 


\section{Appendix Chapter 6}

Supplementary Table 1. List of the different tissue sources and fixation types for every figure and video presented.

1a,b; Supplementary figure 1

1c; 2 ; Supplementary figure $2+3$

3a; 5

3b; 4; Supplementary video 1+2 mouse $\quad 4 \%$ PFA

pig $\quad$ PLP

body donor formalin

autopsy

PLP

Supplementary Video 1. Single sided x-scan of Dil-labelled human amygdala, cleared with hFRUIT and acquired with the diSPIM set-up. The injection site of Dil crystals is located in the upper left corner. Volume dimensions: $400 \times 772 \times 546$ $\mu \mathrm{m}$.

Supplementary Video 2. X-scan from the opposite direction of the same sample. The injection site of Dil crystals is located in the upper right corner. Volume dimensions: $400 \times 772 \times 546 \mu \mathrm{m}$.

Supplementary Video 3. 3D rendering of a deconvolved sub-volume of the data set shown in Supplementary Video 1 and 2. 


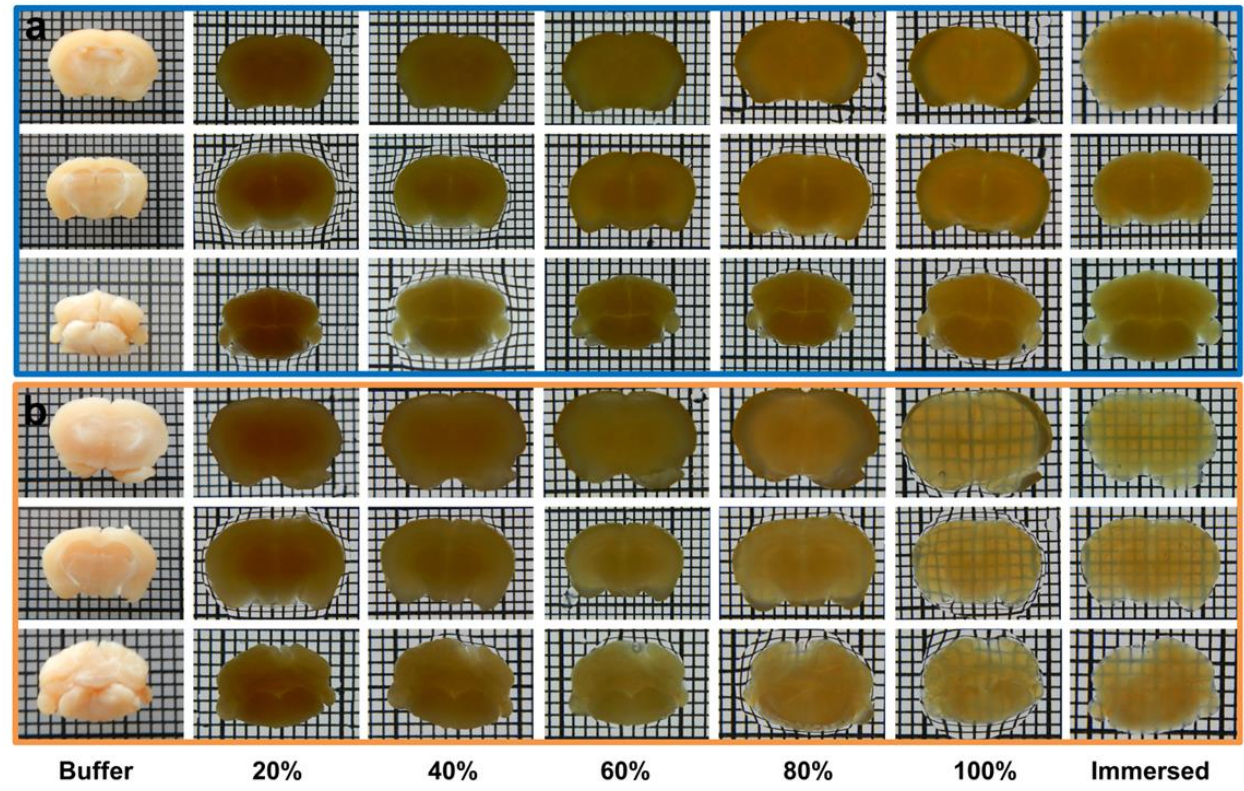

Supplementary Figure 1. (a) FRUIT clearing performance on $3 \mathrm{~mm}$ thick coronal slices of mouse brain and whole mouse cerebellum (bottom row) over ascending series of concentrations. (b) hFRUIT clearing performance on $3 \mathrm{~mm}$ thick coronal mouse brain sections and whole cerebellum (bottom row) for each concentration. Last column shows samples immersed in 1:1 mixture of silicon oil and mineral oil respectively. Grid size 1x1 mm respectively. 


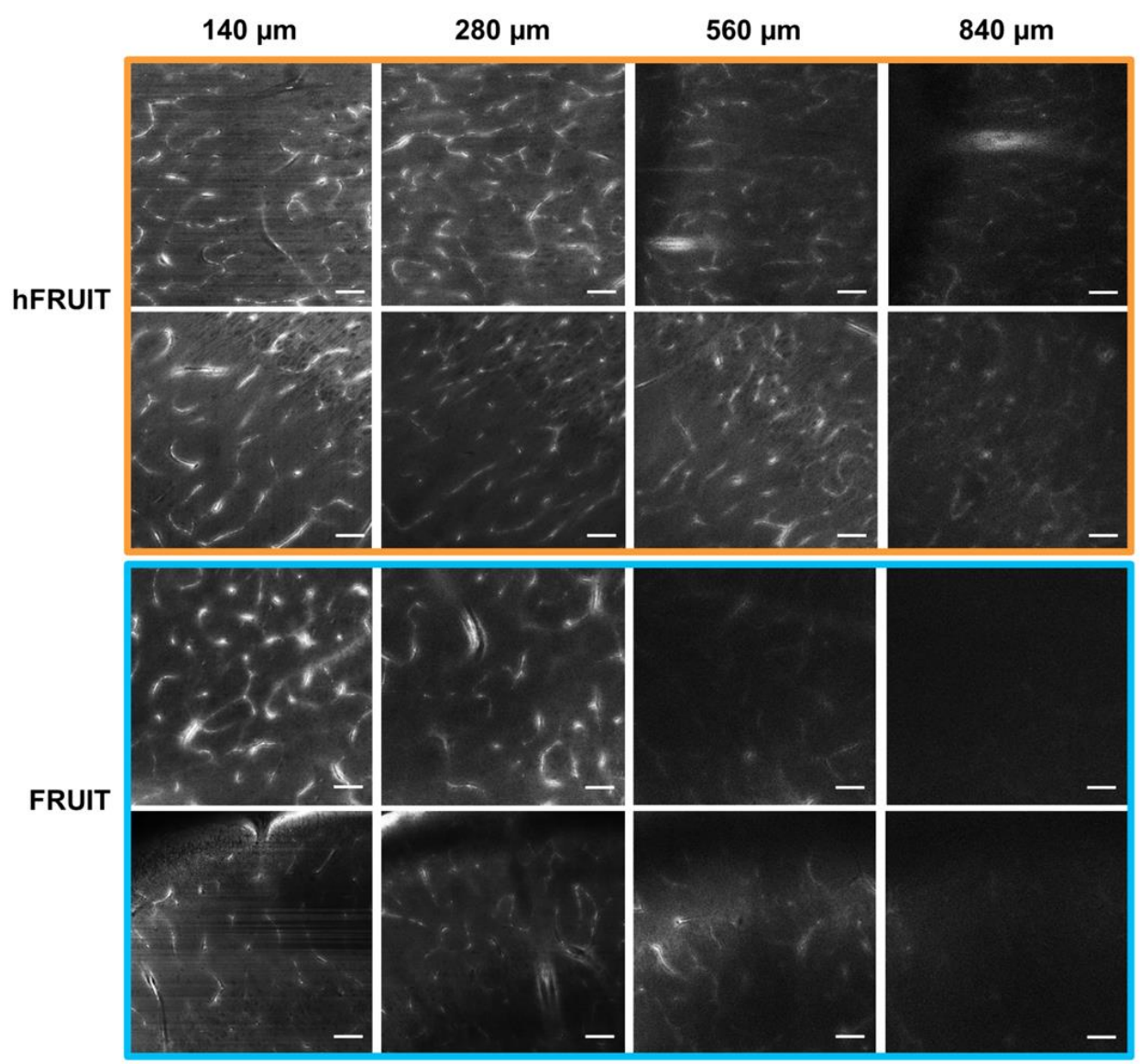

Supplementary Figure 2. Comparison of four representative porcine brain samples, two cleared with hFRUIT (orange) and two FRUIT (blue) respectively. Shown is a qualitative comparison of the autofluorescent blood vessel signal over increasing depths when excited with $488 \mathrm{~nm}$, with each panel adjusted for contrast and brightness (not the signal drop-off over depth). Scale bars: $100 \mu \mathrm{m}$ each. 

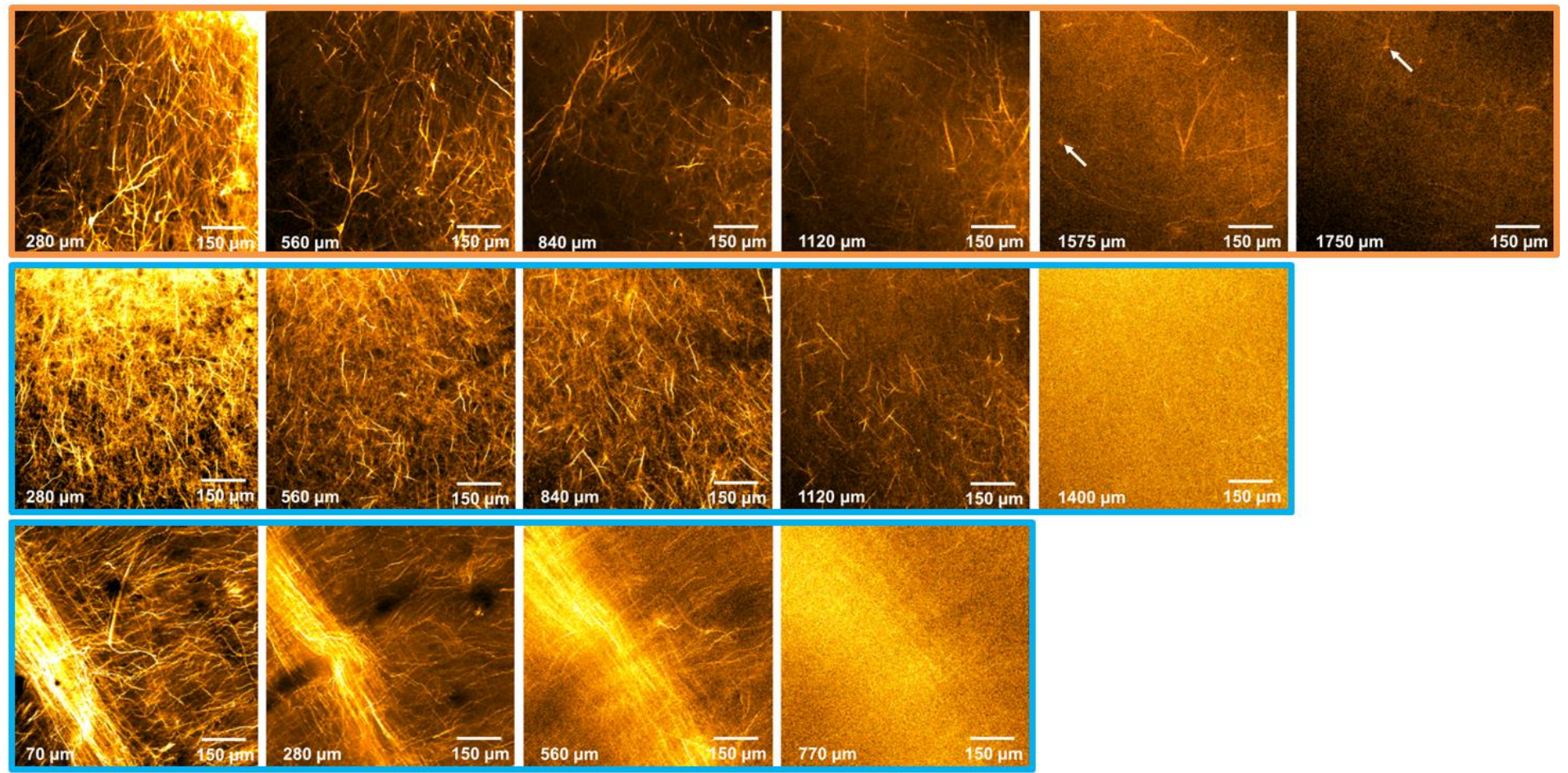

Supplementary Figure 3. Comparison of representative Dil-labelled porcine brain samples cleared with hFRUIT (orange box) or FRUIT (blue boxes) and imaged with CLSM. hFRUIT cleared specimen allowed for deeper imaging of structures in general, with cell bodies being visible up to at least $1500 \mu \mathrm{m}$. 
Chapter 6 - hFRUIT 
Chapter 7 - General Discussion

ChAPTER 7

General Discussion 


\section{Summary of findings}

The overall aim of this thesis was to develop optical clearing and labelling techniques for human brain tissue and 3D imaging of neocortical anatomy and architecture.

In Chapter 2, neocortical tissue of several centimetres in lateral extent and millimetres thick was cleared and labelled for cytoarchitecture with MASH. In this adaptation of the iDISCO+ clearing method, we optimised the protocol for large archival human brain samples up to five millimetres thick. An additional bleaching step was introduced, the delipidation was extended, and more benign solutions for RImatching replaced the original chemical: TDE/CA and WGO/CA. Furthermore, labelling protocols for several classical dyes (e.g. neutral red and methylene blue) were shown to work well as fluorescent cytoarchitecture labels in different spectral bands: MASH-AO (greenyellow), MASH-NR (orange-red), MASH-MB (far-red), MASH-MG (farred). These classic dyes are inexpensive, readily available through several manufacturers, and their small molecular weight enables the deep tissue penetration needed for 3D histology. Hence, this clearing and labelling approach is ideally suited for further scaling up the MASH approach, to even larger samples (Chapter 4). With MASH, we were able to show the typical laminar organisation of V1 and V2 over the entire cortical sheet.

Chapter 3 extended the MASH method by adding a protocol for labelling angioarchitecture resulting in the angioMASH protocol. In 
order to enable the homogenous distribution of the relatively large lectin molecules used as a vessel label, we combined the Harrison protocol $^{1}$ with the SWITCH labelling strategy ${ }^{2}$ and MASH. This new staining can be performed alone or in combination with the cytoarchitecture stainings described in Chapter 2, yielding a double labelling approach for cell bodies (or nuclei if desired) and vasculature. Since we have an insufficient understanding of how the vasculature varies within and between brain areas or individuals, angioMASH offers a great tool to investigate fundamental questions on the complex anatomy of human brain vasculature in future studies. Abnormalities in angioarchitecture can be observed in several neurological diseases and hence this new method has the potential to become widely adopted for studies on neuropathology as well.

At the time of its development, the work in Chapter 2 yielded the largest human brain samples to be cleared and homogeneously labelled (albeit representing only a small fraction of the $\sim 1.5$ litre of total human brain tissue). With the ultimate aim of imaging and reconstructing complete human lobes and eventually entire brains, the original MASH pipeline was further streamlined to process even larger samples than those in Chapter 2. With the new high-throughput version of MASH demonstrated in Chapter 4, it is now feasible to process whole organs in a matter of days. Its potential is demonstrated by simultaneously processing multiple whole coronal slices of human occipital lobes, thereby showcasing the scalability of this new approach. To enable this, we used both commercial SLS and 
conventional FDM/FFF 3D printing to create custom made PP sample holders (designs of which will be made publicly available). The affordability of the MASH method together with the economic rapid prototyping on FDM/FFF printers in particular, would enable smaller labs to engage in large-scale mapping of big mammalian brains or the $3 \mathrm{D}$ investigation of other large organs.

However, histological processing alone is not sufficient to facilitate this structural investigation and mapping of large mammalian brains. A microscope platform on which samples of this size can be mounted and imaged is also necessary. The oblique geometry and stagescanned imaging of the ct-dSPIM introduced in Chapter 5 is ideally suited for imaging extremely large organ slices up to a thickness of 5 $\mathrm{mm}$. We provide a proof of concept for the versatility of oblique lightsheet microscopy, using the ct-dSPIM to image both whole occipital lobe as well as prostate slices. For the latter, the high-throughput version of MASH shown in Chapter 4 was yet again adapted to deparaffinise and rehydrate the thick FFPE prostate samples, thus demonstrating the feasibility of MASH for FFPE tissue and organs other than the brain. The conventional light-sheet geometry used in Chapter 2 and 3, with the illumination directed towards the side of the tissue, limits the lateral size of the sample. Covering substantial parts of large mammalian brains and other organs is therefore not realistically feasible with a classical light-sheet set-up. Hence, for certain neuroscientific or histological questions it is indispensable to use oblique light-sheet microscopes. We expect more laboratories 
will adapt designs such as the ct-dSPIM or similar recently reported microscope set-ups for 3D investigations in healthy and pathological tissue.

In contrast to the other chapters in this thesis, Chapter 6 investigates aqueous clearing solutions for human brain tissue (hFRUIT) instead of the solvent-based MASH. Although the clearing capacity of the hFRUIT protocol is not as high as that of MASH, hFRUIT offers the important compatibility with lipophilic dyes, while delipidating clearing approaches do not. As of writing this, injections of lipophilic dye crystals are the only method available for human brain tissue to investigate intracortical connectivity, at least at the meso- to microscale interface and by using a tracing-like mechanism that ensures transport of label from injection site to connected sites in the vicinity (up to a few centimetres away from the injection site). hFRUIT could, therefore, prove highly valuable to investigate human intracortical circuitry.

\section{Current technical limitations and future directions}

The new developments in the nascent field of 3D histology (the entire process of optical clearing and labelling, volumetric imaging, and 3D image visualisation and analysis) are as rapid as they are exciting. The combination of tissue clearing with volumetric imaging methods, such as the light-sheet microscopy employed in these chapters, is extending into ever more disciplines of the biological sciences ${ }^{3-7}$. Even in neuroscience, where the combination was first introduced, the full 
potential of 3D histological approaches remains untapped. The work in this thesis shows only a part and just the beginning of a burgeoning set of techniques, which will allow the investigation of nervous systems, unprecedented in its coverage, scope, and throughput. In the following paragraphs, I will discuss current methodological limitations and possible future directions of the work presented in this thesis, both for technical improvements as well as neuroscientific applications, which could be ideally targeted with this kind of methodology.

\section{Technological limitations and possible roads to overcome them}

Certainly one of the most obvious limitations in tissue clearing today is the homogeneous labelling of desired structures deep inside the thick sample ${ }^{8,9}$. The actual process of rendering large samples, even whole organs, transparent is now achievable. Highly effective clearing has been demonstrated on a wide variety of tissues and organs ${ }^{6,10-17}$. Therefore, the clearing itself mainly depends on choosing and combining the right steps from the vast literature available. However, in most cases even the most transparent sample is of limited use without highlighting specific structures of interest, although in some instances, imaging the autofluorescence might be sufficient ${ }^{18}$. The easiest way to achieve deep labelling is the use of small organic compounds, which can diffuse quickly and relatively unhindered into the tissue ${ }^{12}$ (see Chapter 2). However, as described in Chapter 1, the use of these dyes can ultimately be limited, depending on the tissue 
type and the diversity of its components. As the labelling depends on rather general chemical interactions with tissue components, it might therefore not be highly specific in a given tissue. Here, old histological works could prove highly insightful, as early histologists were faced with the same problem and could not switch to the more specific, modern probes, such as antibody labels. For some tissue types and specific components therein, there are specific histochemical stainings available, such as elastic fibre stainings ${ }^{19,20}$. Whether or not these old techniques are clearing-compatible and use dyes with fluorescent properties needs to be thoroughly and systematically investigated. However, many questions, especially in very complex structures such as the brain, require highly specific probes for certain targets, e.g. against specific receptors, or neurotransmitters.

Antibodies are by far the most widely used solution to label specific tissue components. However, their application in cleared tissue is still problematic, especially in samples of the dimensions shown in Chapter 4 . The relatively large size of antibodies severely limits their penetration into dense, thick tissues. Although in some cases penetration of several millimetre-sized cubes have been reported ${ }^{21}$, in most instances the penetration is limited to $1 \mathrm{~mm}$ at most ${ }^{2,22-25}$. To overcome this problem, several solutions have been proposed. In order to avoid antibodies getting entangled in the periphery of the sample when initially diffusing into it, SWITCH offers a chemical way to block antibody-antigen binding and allows for a more homogenous label distribution ${ }^{2}$. For the same reason, some protocols suggest using 
very high dilutions of antibodies ${ }^{26}$ and iteratively increasing their concentration (personal communication with Nicolas Renier). However, neither of these methods address the penetration problem per se. To improve the penetration, it is reasonable to remove as many tissue components as possible, which could hinder the diffusion of the label molecules. Therefore, many protocols remove the lipids prior to the labelling step $\mathrm{s}^{2,17,23,27}$ or use chemical permeablisation to loosen up the dense protein network ${ }^{17,23,24,28}$. Furthermore, the softer, gel-like consistency of samples in aqueous and hydrogel-embedding techniques seems to be beneficial for antibody penetration as well compared to the solvent-based clearing methods ${ }^{29}$. Another logical step is the reduction of the molecule's size. Smaller antibody fragments, such as Fab fragments or nano-bodies, diffuse much further into larger specimens ${ }^{30-32}$. Nevertheless, for many of these smaller labels the cost quickly becomes prohibitive for very large samples, at least for now. Furthermore, since these smaller probes are not in common usage, they are available for a limited number of target structures only. In order to enhance the diffusion of large (standard) antibodies, several methods have been proposed. Using an electrical current to pull charged labels quickly through the tissue ${ }^{31,33,34}$, or applying various forms of pressure to push the antibodies through the samples $^{35-39}$ have both been demonstrated to increase label penetration. These methods have their own caveats, such as the maintenance of sample integrity, the ease of implementation of the technique, or the scalability beyond mouse brain sized samples. 
Therefore, despite all the proposed solutions to tackle the antibody problem in tissue clearing, no strategy has been universally adopted yet for samples of several millimetre thickness and most laboratories still struggle to achieve reliable labelling with sufficient quality even on thinner slices. Are there any alternatives to antibodies? Although RNA and peptide technologies such as aptamers offer an exciting avenue for small, specific (and electrically charged) labels, their application in histology is still very limited ${ }^{40,41}$. Once aptamers become widely commercially available at a feasible cost for large tissue volumes, they are a promising candidate to replace antibodies in the context of 3D histology.

Another area of investigation that is currently methodologically limited is the study of intrinsic cortical connectivity in the human brain. As described in Chapter 1 and 5, tracing studies with lipophilic dyes are the only available method today to study this aspect of human neuroanatomy at a microscopic resolution over micro- to mesoscale ranges ${ }^{42-46}$, at least when a tracing-like mechanism that ensures transport of label from injection site to remote connected sites is required. Here, the work on polarised light imaging ${ }^{47-49}$ and optical coherence tomography ${ }^{50-52}$ is duly noted as promising alternative approaches. However, both are local axonal orientation detection techniques, and therefore need computational tractography modelling to infer remote connections (mostly with similar challenges as tractography in macroscale diffusion MRI, unless resolution would allow single axon specificity). Although Chapter 5 and work by a few 
other groups show tentative steps towards 3D investigation of lipophilic tracers ${ }^{23,53,54}$, there are some ways to further improve this approach substantially. The most severe drawback of any published clearing protocol compatible with lipophilic tracers is the limited clearing capacity. Although Chapter 5 shows that fixation can have a significant influence on the clearing performance of a given protocol, changing the fixation routine will not alleviate the inherent RI inhomogeneities in non-delipidated tissue. Hence, the clearing capacity will always be limited and light scattering and aberrations will always be more severe in such samples. Nevertheless, there are at least three potential solutions to address this problem: 1) Using probes with more bathochromic spectra than DiI, the most commonly used tracer. Moving into the far-red to near-infrared spectrum would considerably increase the imaging depth without changing the available clearing protocols ${ }^{55,56}$. It is currently not known however, if dyes such as DiD and DiR would diffuse enough into human post mortem tissue to be suitable as tracers due to their higher molecular weights. 2) The use of fixable dyes would allow the combination with delipidating clearing techniques. This would allow for much deeper imaging as well, provided the label can be retained sufficiently throughout the procedure ${ }^{54}$. Currently there are only a few labels with these properties available and their suitability as tracers in human tissue has not been investigated. 3) The combination of 1) and 2) would obviously provide the ideal solution for this problem, but fixable DiIderivatives in the far-red to near-infrared spectrum are currently not commercially available. 
Despite the limitations with regards to labelling, constant progress is being made in this direction. There is no doubt that the more established 3D histology and optical clearing will become, the more tailor-made probes suitable for deep labelling will become commercially available. Nevertheless, with samples of the size presented in the preceding chapters (specifically in Chapter 4) of many centimetres laterally and millimetres in thickness, an additional limitation becomes the data itself. The data volumes acquired with light-sheet microscopes are well beyond those of conventional fluorescent microscopy. State-of-the-art systems such as the ctdSPIM can write terabytes of data at a rate exceeding the writing speeds of mechanical hard drives. These massive amounts of data do not only have to be saved and stored, but stitched, processed and analysed as well. This is a known challenge since the beginning of light-sheet microscopy ${ }^{57,58}$. This computational problem is only expected to get worse in the next few years, with efforts turning towards high-throughput imaging of ever-larger samples now that suitable imaging platforms, such as the one presented in Chapter 5, are set to become more ubiquitous. In the last few years, many image visualisation and analysis companies turned their attention to this particular issue and several freely available tools have been developed to address some of these issues. Recently solutions have been introduced for multi-view fusion ${ }^{59,60}$, stitching ${ }^{61,62}$, visualisation ${ }^{60,63}$, and compression ${ }^{59,64}$ of light-sheet data, often with the use of deep learning advances. Although the ImageJ environment ${ }^{65}$ combines some of these tools, there is no open-source tool available that 
encompasses every processing step. Since other areas of science have long confronted similar Big Data challenges - be it astronomy, genetics, or many of the "omics" disciplines - one can hope that inspiration can be drawn from these fields to quickly advance data handling in 3D histology. Particularly, more intuitive and end-user friendly solutions targeted at scientists with biomedical rather than computer/data science backgrounds would help to bring 3D histological acquisition and analysis within reach of more laboratories and researchers.

\section{Future applications of optical clearing}

In the field of neuroscience, the optical clearing and 3D investigation of murine brains is already rapidly becoming a standard technique. It seems highly likely that increasing use will be made of this technique in human neuroanatomy as well. Since tissue clearing can be combined with such a vast variety of other methods, such as in situ hybridisation $^{2,66,67}$, viral tracing ${ }^{68,69}$, or trans-cranial imaging ${ }^{5,7}$, I will focus on possible uses in human tissue only. Also, the examples given below are by no means exhaustive. The logical immediate targets for future 3D histological studies in the human brain are relatively small yet contain inherently 3D features, the aspects of which are difficult to study in 2D brain sections.

One such example in the neocortex are columnar structures, be they minicolumns comprising a few dozen neurons, or larger macrocolumns more obviously related to different functional aspects 
e.g. orientation selectivity in the visual cortex ${ }^{70-73}$. Distinguishing the different neuron types and fibre populations in these structures would greatly improve our fundamental understanding of human cortical neuroanatomy. The MASH method, ideally with the addition of antibody stainings directed at specific neuronal populations or components, provides a promising platform for the study of minicolumns. Tracer studies with lipophilic dyes on the other hand reveal a patchy cortical connectivity likely related to the larger functional macrocolumns ${ }^{42,44}$. Hence, when combined with hFRUIT these features could also be investigated in 3D. The potential methodological improvements discussed in Chapter 5 and those discussed above, could eventually allow the combination of MASH and hFRUIT in the future.

Another potential target are relatively small neuron populations, localised in subcortical structures ${ }^{28,36,74}$. Tissue clearing offers the possibility to study deep nuclei in their entirety, counting their whole cell populations without stereological bias and potentially stain multiple components simultaneously and/or iteratively to systematically characterise these systems.

Ultimately, these applications could be brought to much larger tissue samples, as the pilot study in Chapter 4 has shown. Once the necessary infrastructure is in place, mesoscopic datasets of various neuroanatomical features could be acquired at an unprecedented rate. This would open the door to the creation of brain maps 
complementary to ongoing large-scale initiatives such as the Jülich Brain Atlas $^{75}$ or the Allen Human Brain Atlas $^{76,77}$, which have been transformative as modern reference atlases. One advantage of the 3D histology pipelines presented in this thesis is that they are much less labour intensive to cover a certain volume of brain tissue, as compared to modern high-throughput 2D histology. For this reason, it is believed that the creation of these types of atlases could be achieved with less manpower and hence even in smaller laboratories with the aid of 3D histology. This is of course not to say that projects with the ambition of the above-mentioned large imaging initiatives can be simply replaced by small groups. It would rather empower smaller laboratories with very specific research questions in mind, to engage in brain mapping and anatomical investigations, which would not have been possible with such a work force or on a similar timescale before. In a complementary way, it might enable dedicated large institutes and labs to pursue higher- $\mathrm{N}$ brain mapping to characterise individual variation and large group or population level phenotyping.

Since optical clearing and light-sheet microscopy provide microscopic resolution at mesoscopic FOVs, these methods are also ideally suited for the generation of multi-modal data sets with techniques that overlap in terms of either FOV or resolution. Nondelipidating clearing approaches such as hFRUIT might be combined with high-throughput EM methods ${ }^{78}$ to cover the micro- and nanoscale end of the spectrum. The combination of expansion microscopy ${ }^{79-82}$, a rapidly evolving set of clearing techniques, with 
conventional tissue clearing methods could serve a similar purpose. In expansion microscopy, a fluorescent probe is kept in position by a chemical scaffold while the protein connections in the tissue are digested. Once these tissue hybrids are exposed to water, the samples will swell up to several times their original size while the relative positions of the fluorescent labels are retained. This way, the distance between the label molecules is increased and the effective resolution can be enhanced beyond the diffraction limit of conventional light microscopy. The diffraction limit would ultimately restrict the resolution when applied to the non-expanded sample (unless other super-resolution microscopy techniques are applied). Alternatively, there are many imaging techniques which operate at larger FOVs, such as UHF MRI (MRI $>=7 T)^{46,83-85}$ or Optical Coherence Tomography $(\mathrm{OCT})^{50,52,86}$. UHF MRI can approach the lower resolution end of the mesoscopic spectrum in vivo (still many hundreds of $\mu \mathrm{m}$ ) ${ }^{84,87}$, but in post mortem imaging, much higher resolutions are possible ${ }^{88-91}$. In the case of the human brain (or other very large mammalian brains) the non-destructive nature of post mortem UHF MRI offers the unique advantage of obtaining a non-dissected reference dataset for any 3D histological investigations. The different datasets could be related to one another because of their overlapping FOV and resolution and hence give a more macro-anatomical context to the microscopy data. Vice versa, the specificity of the applied labels in 3D histology offers a great way to validate structures observed in the other, more indirect methodology. 
Lastly, the study of brain pathologies could immensely benefit from these techniques. It has already been shown that the visualisation of plaques in Alzheimer's disease in 3D can highlight clustering of these structures which would be easily missed with conventional histology ${ }^{21}$. Investigations into such spatial aspects of brain pathologies could aid our understanding of the underlying mechanisms of those diseases and their progression. Pathologies, which are highly localised, are well suited for the combination with 3D histology in particular, for the same reasons as stated above: small, localised areas could be viewed in their entirety while revealing spatial features that might be lost or difficult to detect in 2D histology. One example for this would be focal cortical dysplasia (FCD), a leading cause of epilepsy in adults. As the name implies, patients with this disease show highly localized parts of cortical tissue with unusual vertical and/or horizontal architecture ${ }^{92-}$ 94. Specifically, FCD type Ia displays an abnormal columnar organisation, while type Ib shows an abnormal organisation of the 6 cortical layers. Type Ic exhibits both abnormal radial and laminar organisation. All type I forms typically produce lesions in the temporal lobe and the onset of the symptoms appears later in patients as compared to type $\mathrm{II}^{95}$. The visualisation of entire biopsies from FCD patients could have huge implications for revealing the precise structural changes and their causes in this disease. Outside the brain, tissue clearing is already used for 3D pathology of multiple organs, usually in the context of cancer biopsies ${ }^{10,12,18,96}$. In these cases, imaging the entirety of the sample, as explored for prostate tissue in Chapter 5 , can help to classify cancer stages, because 3D pathology 
overcomes the potential stereological bias. This could ultimately have huge implications for patients, whose treatment regimens change because of this.

\section{Concluding remarks}

The widespread use of optical clearing and light-sheet microscopy on human tissue as a standard technique is still some years away at least. Nevertheless, their disruptive potential for fields focusing on structural questions, such as anatomy and histology, is undeniable. Once the technical limitations of these still relatively new methods are properly addressed, ultimately many aspects of conventional histological studies could be either replaced or at least augmented, supplemented and enhanced with 3D histology. This would apply beyond the study of nervous tissue, to any tissue, be it animal or plant $^{6}$. Even large organs ${ }^{17}$ and entire bodies (albeit of small mammals) $)^{14,15,97}$ have already been cleared. With more innovative solutions to labelling, imaging and analysis, microscopic investigations of whole human organs might therefore become possible in the near future. Although not all necessary steps for a complete 3D histology pipeline are equally advanced as the optical clearing itself, I consider it only a matter of time until these last hurdles are overcome. Therefore, these methods have the capability to revitalize the interest in traditional fields of morphological studies in general. 


\section{References}

1 Harrison, C. H. et al. A novel method to visualise the three-dimensional organisation of the human cerebral cortical vasculature. Journal of anatomy 232, 1025-1030, doi:10.1111/joa.12805 (2018).

2 Murray, E. et al. Simple, Scalable Proteomic Imaging for HighDimensional Profiling of Intact Systems. Cell 163, 1500-1514, doi:10.1016/j.cell.2015.11.025 (2015).

3 Kagami, K., Shinmyo, Y., Ono, M., Kawasaki, H. \& Fujiwara, H. Threedimensional evaluation of murine ovarian follicles using a modified CUBIC tissue clearing method. Reproductive biology and endocrinology : RB\&E 16, 72, doi:10.1186/s12958-018-0381-7 (2018).

4 Lesage, M. et al. C-ECi: a CUBIC-ECi combined clearing method for three-dimensional follicular content analysis in the fish ovary†. Biology of Reproduction 103, 1099-1109, doi:10.1093/biolre/ioaa142 (2020).

5 Costantini, I., Cicchi, R., Silvestri, L., Vanzi, F. \& Pavone, F. S. In-vivo and ex-vivo optical clearing methods for biological tissues: review. Biomedical optics express 10, 5251-5267, doi:10.1364/BOE.10.005251 (2019).

6 Palmer, W. M. et al. PEA-CLARITY: 3D molecular imaging of whole plant organs. Scientific reports 5, 13492, doi:10.1038/srep13492 (2015).

7 Zhao, Y.-J. et al. Skull optical clearing window for in vivo imaging of the mouse cortex at synaptic resolution. Light: Science \& Applications 7, 17153-17153, doi:10.1038/lsa.2017.153 (2018).

8 Lai, H. M., Ng, W. L., Gentleman, S. M. \& Wu, W. Chemical Probes for Visualizing Intact Animal and Human Brain Tissue. Cell chemical biology 24, 659-672, doi:10.1016/j.chembiol.2017.05.015 (2017).

9 Tainaka, K., Kuno, A., Kubota, S. I., Murakami, T. \& Ueda, H. R. Chemical Principles in Tissue Clearing and Staining Protocols for WholeBody Cell Profiling. Annual review of cell and developmental biology 32, 713-741, doi:10.1146/annurev-cellbio-111315-125001 (2016).

10 Glaser, A. K. et al. Light-sheet microscopy for slide-free non-destructive pathology of large clinical specimens. Nature biomedical engineering $\mathbf{1}$, doi:10.1038/s41551-017-0084 (2017). 
11 Lesage, M. et al. C-Eci: A CUBIC-Eci COMBINED CLEARING METHOD FOR 3D FOLLICULAR CONTENT ANALYSIS IN THE FISH OVARY (bioRxiv, 2020).

12 Nojima, S. et al. CUBIC pathology: three-dimensional imaging for pathological diagnosis. Scientific reports 7, 9269, doi:10.1038/s41598017-09117-0 (2017).

13 Scott, G. D., Blum, E. D., Fryer, A. D. \& Jacoby, D. B. Tissue optical clearing, three-dimensional imaging, and computer morphometry in whole mouse lungs and human airways. American journal of respiratory cell and molecular biology 51, 43-55, doi:10.1165/rcmb.2013-0284OC (2014).

14 Tainaka, K. et al. Whole-body imaging with single-cell resolution by tissue decolourization. Cell 159, 911-924, doi:10.1016/j.cell.2014.10.034 (2014).

15 Treweek, J. B. et al. Whole-body tissue stabilization and selective extractions via tissue-hydrogel hybrids for high-resolution intact circuit mapping and phenotyping. Nature protocols 10, 1860-1896, doi:10.1038/nprot.2015.122 (2015).

16 Zhang, W. L. et al. Skeletal Muscle CLARITY: A Preliminary Study of Imaging The Three-Dimensional Architecture of Blood Vessels and Neurons. Cell journal 20, 132-137, doi:10.22074/cellj.2018.5266 (2018).

17 Zhao, S. et al. Cellular and Molecular Probing of Intact Human Organs. Cell 180, 796-812 e719, doi:10.1016/j.cell.2020.01.030 (2020).

18 Sabdyusheva Litschauer, I. et al. 3D histopathology of human tumours by fast clearing and ultramicroscopy. Scientific reports 10, 17619, doi:10.1038/s41598-020-71737-w (2020).

19 Kiernan, J. A. Histological and Histochemical Methods: Theory and Practice. (Scion, 2015).

20 Mulisch, M. \& Welsch, U. Romeis - Mikroskopische Technik. (Springer Berlin Heidelberg, 2015).

21 Liebmann, T. et al. Three-Dimensional Study of Alzheimer's Disease Hallmarks Using the iDISCO Clearing Method. Cell reports 16, 11381152, doi:10.1016/j.celrep.2016.06.060 (2016). 
22 Chung, K. et al. Structural and molecular interrogation of intact biological systems. Nature 497, 332-337, doi:10.1038/nature12107 (2013).

23 Lai, H. M. et al. Next generation histology methods for three-dimensional imaging of fresh and archival human brain tissues. Nature communications 9, 1066, doi:10.1038/s41467-018-03359-w (2018).

24 Liu, A. K. et al. Bringing CLARITY to the human brain: visualisation of Lewy pathology in three dimensions. Neuropathology and applied neurobiology 42, 573-587, doi:10.1111/nan.12293 (2016).

25 Liu, A. K. L., Lai, H. M., Chang, R. C. C. \& Gentleman, S. M. Free of acrylamide sodium dodecyl sulphate (SDS)-based tissue clearing (FASTClear): a novel protocol of tissue clearing for three-dimensional visualisation of human brain tissues. Neuropathology and applied neurobiology 43, 346-351, doi:https://doi.org/10.1111/nan.12361 (2017).

26 Susaki, E. A. et al. Versatile whole-organ/body staining and imaging based on electrolyte-gel properties of biological tissues. Nature communications 11, 1982, doi:10.1038/s41467-020-15906-5 (2020).

27 Tainaka, K. et al. Chemical Landscape for Tissue Clearing Based on Hydrophilic Reagents. Cell reports 24, 2196-2210 e2199, doi:10.1016/j.celrep.2018.07.056 (2018).

28 Renier, N. et al. iDISCO: a simple, rapid method to immunolabel large tissue samples for volume imaging. Cell 159, 896-910, doi:10.1016/j.cell.2014.10.010 (2014).

29 Morawski, M. et al. Developing 3D microscopy with CLARITY on human brain tissue: Towards a tool for informing and validating MRI-based histology. Neuroimage 182, 417-428, doi:10.1016/j.neuroimage.2017.11.060 (2018).

30 Cai, R. et al. Panoptic imaging of transparent mice reveals whole-body neuronal projections and skull-meninges connections. Nature neuroscience 22, 317-327, doi:10.1038/s41593-018-0301-3 (2019).

31 Li, J., Czajkowsky, D. M., Li, X. \& Shao, Z. Fast immuno-labeling by electrophoretically driven infiltration for intact tissue imaging. Scientific reports 5, 10640, doi:10.1038/srep10640 (2015). 
32 Pan, C. et al. Deep Learning Reveals Cancer Metastasis and Therapeutic Antibody Targeting in the Entire Body. Cell 179, 16611676.e1619, doi:https://doi.org/10.1016/j.cell.2019.11.013 (2019).

33 Kim, S.-Y. et al. Stochastic electrotransport selectively enhances the transport of highly electromobile molecules. Proceedings of the National Academy of Sciences 112, E6274, doi:10.1073/pnas.1510133112 (2015).

34 Yun, D. H. et al. Ultrafast immunostaining of organ-scale tissues for scalable proteomic phenotyping. bioRxiv, 660373, doi:10.1101/660373 (2019).

$35 \mathrm{Ku}, \mathrm{T}$. et al. Elasticizing tissues for reversible shape transformation and accelerated molecular labeling. Nature methods 17, 609-613, doi:10.1038/s41592-020-0823-y (2020).

36 Du, H., Hou, P., Wang, L., Wang, Z. \& Li, Q. Modified CLARITY Achieving Faster and Better Intact Mouse Brain Clearing and Immunostaining. Scientific reports 9, 10571, doi:10.1038/s41598-019-46814-4 (2019).

37 Fiorelli, R. et al. Enhanced tissue penetration of antibodies through pressurized immunohistochemistry. bioRxiv, 2020.2009.2025.311936, doi:10.1101/2020.09.25.311936 (2020).

38 Kim, D. H. et al. Tissue-Clearing Technique and Cutaneous Nerve Biopsies: Quantification of the Intraepidermal Nerve-Fiber Density Using Active Clarity Technique-Pressure Related Efficient and Stable Transfer of Macromolecules Into Organs. J Clin Neurol 15, 537-544 (2019).

39 Lee, E. et al. ACT-PRESTO: Rapid and consistent tissue clearing and labeling method for 3-dimensional (3D) imaging. Scientific reports 6 , 18631, doi:10.1038/srep18631 (2016).

40 Bauer, M., Macdonald, J., Henri, J., Duan, W. \& Shigdar, S. The Application of Aptamers for Immunohistochemistry. Nucleic Acid Therapeutics 26, 120-126, doi:10.1089/nat.2015.0569 (2016).

$41 \mathrm{Pu}, \mathrm{Y}$. et al. Using DNA Aptamer Probe for Immunostaining of Cancer Frozen Tissues. Analytical Chemistry 87, 1919-1924, doi:10.1021/ac504175h (2015). 
42 Burkhalter, A. \& Bernardo, K. L. Organisation of corticocortical connections in human visual cortex. Proceedings of the National Academy of Sciences 86, 1071, doi:10.1073/pnas.86.3.1071 (1989).

43 Burkhalter, A., Bernardo, K. L. \& Charles, V. Development of local circuits in human visual cortex. The Journal of Neuroscience 13, 1916, doi:10.1523/JNEUROSCI.13-05-01916.1993 (1993).

44 Galuske, R. A. W., Schlote, W., Bratzke, H. \& Singer, W. Interhemispheric Asymmetries of the Modular Structure in Human Temporal Cortex. Science 289, 1946, doi:10.1126/science.289.5486.1946 (2000).

45 Lanciego, J. L. \& Wouterlood, F. G. A half century of experimental neuroanatomical tracing. Journal of chemical neuroanatomy 42, 157183, doi:10.1016/j.jchemneu.2011.07.001 (2011).

46 Seehaus, A. K. et al. Histological validation of DW-MRI tractography in human postmortem tissue. Cerebral cortex 23, 442-450, doi:10.1093/cercor/bhs036 (2013).

47 Axer, $\mathrm{H}$. et al. Microstructural Analysis of Human White Matter Architecture Using Polarized Light Imaging: Views from Neuroanatomy. Frontiers in neuroinformatics 5, 28 (2011).

48 Axer, M. et al. High-Resolution Fiber Tract Reconstruction in the Human Brain by Means of Three-Dimensional Polarized Light Imaging. Frontiers in neuroinformatics 5, 34 (2011).

49 Axer, M. et al. Estimating Fiber Orientation Distribution Functions in 3DPolarized Light Imaging. Frontiers in neuroanatomy 10, 40 (2016).

50 Juliette Ben, A. et al. Single myelin fiber imaging in living rodents without labeling by deep optical coherence microscopy. Journal of biomedical optics 16, 1-10, doi:10.1117/1.3650770 (2011).

51 Magnain, C. et al. Blockface histology with optical coherence tomography: A comparison with Nissl staining. Neurolmage 84, 524-533, doi:https://doi.org/10.1016/j.neuroimage.2013.08.072 (2014).

52 Ren, J., Choi, H., Chung, K. \& Bouma, B. E. Label-free volumetric optical imaging of intact murine brains. Scientific reports 7, 46306, doi:10.1038/srep46306 (2017). 
$53 \mathrm{Hou}, \mathrm{B}$. et al. Scalable and Dil-compatible optical clearance of the mammalian brain. Frontiers in neuroanatomy 9, 19, doi:10.3389/fnana.2015.00019 (2015).

54 Jensen, K. H. \& Berg, R. W. CLARITY-compatible lipophilic dyes for electrode marking and neuronal tracing. Scientific reports 6, 32674, doi:10.1038/srep32674 (2016).

55 Smith, A. M., Mancini, M. C. \& Nie, S. Second window for in vivo imaging. Nature Nanotechnology 4, 710-711, doi:10.1038/nnano.2009.326 (2009).

56 Weissleder, R. A clearer vision for in vivo imaging. Nature biotechnology 19, 316-317, doi:10.1038/86684 (2001).

57 Reynaud, E. G., Krzic, U., Greger, K. \& Stelzer, E. H. Light sheet-based fluorescence microscopy: more dimensions, more photons, and less photodamage. HFSP journal 2, 266-275, doi:10.2976/1.2974980 (2008).

58 Reynaud, E. G., Peychl, J., Huisken, J. \& Tomancak, P. Guide to lightsheet microscopy for adventurous biologists. Nature methods 12, 30-34, doi:10.1038/nmeth.3222 (2015).

59 Amat, F. et al. Efficient processing and analysis of large-scale light-sheet microscopy data. Nature protocols 10, 1679-1696, doi:10.1038/nprot.2015.111 (2015).

60 Pietzsch, T., Saalfeld, S., Preibisch, S. \& Tomancak, P. BigDataViewer: visualisation and processing for large image data sets. Nature methods 12, 481-483, doi:10.1038/nmeth.3392 (2015).

61 Bria, A. \& lannello, G. TeraStitcher - A tool for fast automatic 3D-stitching of teravoxel-sized microscopy images. BMC Bioinformatics 13, 316, doi:10.1186/1471-2105-13-316 (2012).

62 Hörl, D. et al. BigStitcher: reconstructing high-resolution image datasets of cleared and expanded samples. Nature methods 16, 870-874, doi:10.1038/s41592-019-0501-0 (2019).

63 Royer, L. A. et al. ClearVolume: open-source live 3D visualisation for light-sheet microscopy. Nature methods 12, 480-481, doi:10.1038/nmeth.3372 (2015). 
64 Balázs, B., Deschamps, J., Albert, M., Ries, J. \& Hufnagel, L. A real-time compression library for microscopy images. bioRxiv, 164624, doi:10.1101/164624 (2017).

65 Schindelin, J. et al. Fiji: an open-source platform for biological-image analysis. Nature methods 9, 676-682, doi:10.1038/nmeth.2019 (2012).

66 Moffitt, J. R. et al. High-performance multiplexed fluorescence in situ hybridization in culture and tissue with matrix imprinting and clearing. Proceedings of the National Academy of Sciences 113, 14456, doi:10.1073/pnas.1617699113 (2016).

67 Shah, S. et al. Single-molecule RNA detection at depth by hybridization chain reaction and tissue hydrogel embedding and clearing. Development 143, 2862-2867, doi:10.1242/dev.138560 (2016).

68 Economo, M. N. et al. Distinct descending motor cortex pathways and their roles in movement. Nature 563, 79-84, doi:10.1038/s41586-0180642-9 (2018).

69 Economo, M. N., Winnubst, J., Bas, E., Ferreira, T. A. \& Chandrashekar, J. Single-neuron axonal reconstruction: The search for a wiring diagram of the brain. The Journal of comparative neurology 527, 2190-2199, doi:10.1002/cne.24674 (2019).

70 Molnár, Z. \& Rockland, K. S. Cortical columns. 103-126, doi:10.1016/b978-0-12-814411-4.00005-6 (2020).

71 Mountcastle, V. B. The columnar organisation of the neocortex. Brain : a journal of neurology 120, 701-722, doi:10.1093/brain/120.4.701 (1997).

72 Peters, A. \& Sethares, C. Myelinated axons and the pyramidal cell modules in monkey primary visual cortex. Journal of Comparative Neurology 365, 232-255, doi:https://doi.org/10.1002/(SICl)10969861(19960205)365:2<232::AID-CNE3>3.0.CO;2-6 (1996).

73 Rockland, K. S. Five points on columns. Frontiers in neuroanatomy 4, 22, doi:10.3389/fnana.2010.00022 (2010).

74 Renier, N. et al. Mapping of Brain Activity by Automated Volume Analysis of Immediate Early Genes. Cell 165, 1789-1802, doi:10.1016/j.cell.2016.05.007 (2016). 
75 Amunts, K., Mohlberg, H., Bludau, S. \& Zilles, K. Julich-Brain: A 3D probabilistic atlas of the human brain's cytoarchitecture. Science 369 , 988, doi:10.1126/science.abb4588 (2020).

76 Hawrylycz, M. J. et al. An anatomically comprehensive atlas of the adult human brain transcriptome. Nature 489, 391-399, doi:10.1038/nature11405 (2012).

77 Miller, J. A. et al. Transcriptional landscape of the prenatal human brain. Nature 508, 199-206, doi:10.1038/nature13185 (2014).

78 Shapson-Coe, A. et al. A connectomic study of a petascale fragment of human cerebral cortex. bioRxiv, 2021.2005.2029.446289, doi:10.1101/2021.05.29.446289 (2021).

79 Gao, R. et al. Cortical column and whole-brain imaging with molecular contrast and nanoscale resolution. Science $\mathbf{3 6 3}$, doi:10.1126/science.aau8302 (2019).

$80 \mathrm{Ku}, \mathrm{T}$. et al. Multiplexed and scalable super-resolution imaging of threedimensional protein localization in size-adjustable tissues. Nature biotechnology 34, 973-981, doi:10.1038/nbt.3641 (2016).

81 Murakami, T. C. et al. A three-dimensional single-cell-resolution wholebrain atlas using CUBIC-X expansion microscopy and tissue clearing. Nature neuroscience 21, 625-637, doi:10.1038/s41593-018-0109-1 (2018).

82 Tillberg, P. W. et al. Protein-retention expansion microscopy of cells and tissues labeled using standard fluorescent proteins and antibodies. Nature biotechnology 34, 987-992, doi:10.1038/nbt.3625 (2016).

83 Alkemade, A. et al. 7 Tesla MRI Followed by Histological 3D Reconstructions in Whole-Brain Specimens. Frontiers in neuroanatomy 14, 68 (2020).

84 Dumoulin, S. O., Fracasso, A., van der Zwaag, W., Siero, J. C. W. \& Petridou, N. Ultra-high field MRI: Advancing systems neuroscience towards mesoscopic human brain function. Neurolmage 168, 345-357, doi:https://doi.org/10.1016/j.neuroimage.2017.01.028 (2018).

85 Seehaus, A. et al. Histological validation of high-resolution DTI in human post mortem tissue. Frontiers in neuroanatomy 9, 98, doi:10.3389/fnana.2015.00098 (2015). 
86 Magnain, C. et al. Blockface histology with optical coherence tomography: a comparison with Nissl staining. Neuroimage 84, 524-533, doi:10.1016/j.neuroimage.2013.08.072 (2014).

87 Lüsebrink, F. et al. Comprehensive ultrahigh resolution whole brain in vivo MRI dataset as a human phantom. Scientific Data 8, 138, doi:10.1038/s41597-021-00923-w (2021).

88 Edlow, B. L. et al. 7 Tesla MRI of the ex vivo human brain at 100 micron resolution. Scientific Data 6, 244, doi:10.1038/s41597-019-0254-8 (2019).

89 Fritz, F. J. et al. Ultra-high resolution and multi-shell diffusion MRI of intact ex vivo human brains using kT-dSTEAM at 9.4T. Neurolmage 202, 116087, doi:https://doi.org/10.1016/j.neuroimage.2019.116087 (2019).

90 Sengupta, S. et al. High resolution anatomical and quantitative MRI of the entire human occipital lobe ex vivo at 9.4T. Neurolmage 168, 162171, doi:https://doi.org/10.1016/j.neuroimage.2017.03.039 (2018).

91 Roebroeck, A., Miller, K. L. \& Aggarwal, M. Ex vivo diffusion MRI of the human brain: Technical challenges and recent advances. NMR in Biomedicine 32, e3941, doi:https://doi.org/10.1002/nbm.3941 (2019).

92 Blümcke, I. et al. The clinicopathologic spectrum of focal cortical dysplasias: A consensus classification proposed by an ad hoc Task Force of the ILAE Diagnostic Methods Commission1. Epilepsia 52, 158174, doi:https://doi.org/10.1111/j.1528-1167.2010.02777.x (2011).

93 Garbelli, R. et al. Architectural (Type IA) Focal Cortical Dysplasia and Parvalbumin Immunostaining in Temporal Lobe Epilepsy. Epilepsia 47, 1074-1078, doi:https://doi.org/10.1111/j.1528-1167.2006.00577.x (2006).

94 Krsek, P. et al. Different features of histopathological subtypes of pediatric focal cortical dysplasia. Annals of neurology 63, 758-769, doi:https://doi.org/10.1002/ana.21398 (2008).

95 Kabat, J. \& Król, P. Focal cortical dysplasia - review. Pol J Radio/ 77, 3543, doi:10.12659/pjr.882968 (2012).

96 Glaser, A. K. et al. Multi-immersion open-top light-sheet microscope for high-throughput imaging of cleared tissues. Nature communications 10, 2781, doi:10.1038/s41467-019-10534-0 (2019). 
97 Pan, C. et al. Shrinkage-mediated imaging of entire organs and organisms using uDISCO. Nature methods 13, 859-867, doi:10.1038/nmeth.3964 (2016). 
Chapter 7 - General Discussion 


\section{Impact Paragraph}

The main objective of this thesis was the development of methods for 3D imaging in the field of histology (the investigation of tissues), with large intact tissue blocks of the human brain being the main investigative focus. In traditional histological methods, 2D images of thin slices are either individually interpreted, or retrospectively reconstructed in 3D. In contrast, the nascent field of 3D histology focuses on the immediate acquisition of 3D information over large fields of view. The aim is to slice the tissue as little as possible and in this way preserve the structures of interest within it. This thesis focusses mainly on the primary steps necessary for such an endeavour: 1) optical tissue clearing, i.e. the transformation of the opaque tissue into a transparent histological sample that can be microscopically imaged in 3D, 2) the reliable labelling of thick, large pieces of tissue, and 3) the volumetric microscopy necessary to image these transparent samples over large fields of views. This thesis provides several improvements towards such 3D histological investigations in the human brain. In this impact paragraph, the dissemination, scientific and commercial applications, and wider academic and clinical translation potential is briefly set out. This is split into MASH, ct-dSPIM, and hFRUIT, as these are the three central methodological developments of this thesis. 


\section{MASH}

The MASH (Multiscale Architectonic Staining of Human cortex) clearing and labelling method has been primarily deployed in this thesis to visualize cytoarchitecture (the organisation of cell bodies; Chapters 2, 4, and 5) as well as angioarchitecture (the organisation of blood vessels; Chapter 3) in the healthy human brain. As such, MASH is a useful tool for fundamental neuroscientific investigations of brain architecture and brain mapping. In particular, the combination of a high-throughput MASH pipeline for very large samples (Chapter 4) with the mesoscopic imaging capacities of the ct-dSPIM microscope set-up (Chapter 5) is well suited for human brain mapping (and the brains of other large mammals).

\section{Dissemination and current applications}

The original MASH protocol was first disseminated at the Society for Neuroscience (SfN) and Light Sheet Fluorescence Microscopy (LSFM) conferences in 2017 and is widely available as an open access publication $^{1}$. The high quality of tissue clearing achievable with this method has generated a lot of attention within the tissue clearing community and helped to initiate the ongoing collaboration with the Helmchen laboratory in Zürich (results of which are reported in Chapter 3 and in the recent publication on the mesoSPIM microscope system $^{2}$ ). Furthermore, it has caught the attention of both academic as well as industrial light-sheet microscope developers. Large MASHprocessed samples have been provided to Dr. Fabian Voigt (former 
member of the Helmchen laboratory) and Björn Eismann (Luxendo GmbH, Heidelberg, Germany) for tests on proof-of-principle lightsheet set-ups, optimized for very large cleared samples, demonstrating the commercial interest in such large-scale systems. The angioMASH protocol has been presented as a selected talk at the LSFM conference 2020 and the detailed protocol will be made available as an open access publication. Likewise, it is planned that all data obtained in this study will be made available on online repositories. Similarly, the high-throughput MASH protocol has been presented in poster form at the Swiss Light-Sheet Microscopy Workshop 2019 as well as at the SfN and LSFM conference of the preceding year. Protocols as well as files for the 3D pintable hardware will be made openly available both upon publication and in online repositories. Additionally, MASH has been presented repeatedly locally at Maastricht University and its clearing capacity was explored on highly myelinated, white matter rich samples. Specifically, rat and human spinal cord samples (in collaboration with the School for Mental Health and Neuroscience (MHeNs) and the Department of Urology, respectively) as well as peripheral human nerve samples (in collaboration with the Department of Anatomy and Embryology) have been successfully cleared with MASH in several pilots.

\section{Wider scientific and academic potential}

Beyond the reported cytoarchitecture and angioarchitecture mapping in the human brain and their further development, other 
areas of fundamental neuroscience could also benefit from MASH and 3D histology in general. Tracer studies, in which live animals (or more rarely post mortem human tissue, see Chapter 6) are injected with a tracer to label sparse axon populations, either originating or terminating at a specific site of interest, are one example. With classical 2D histology, the axon segments contained in each thin tissue slice have to be reconstructed in $3 \mathrm{D}$. This is a very labour intensive and error prone method, as individual axon segments have to be identified and the likelihood of losing traced axons over the consecutive sections is high, especially when they are very sparse. Optical clearing in general offers a way to visualise entire brains of smaller mammals such as mice ${ }^{3-5}$, $\operatorname{rats}^{6,7}$, and even marmoset monkeys ${ }^{8}$. Since there is no need for sectioning, the traced connections can be identified more robustly ${ }^{9,10}$. MASH offers the additional advantage of providing several dyes to counterstain the brain's cytoarchitecture and hence offers more anatomical context, given that the tracers can be retained in the tissue in an organicsolvent-compatible manner.

Volumetric imaging of large samples cleared with MASH provides a promising way for the validation of other imaging methodologies such as magnetic resonance imaging $(\mathrm{MRI})^{11}$ and near infra-red spectroscopy (NIRS) ${ }^{12,13}$. In the case of MRI, especially acquisitions at ultra-high field (UHF; field strengths of 7T and more) ${ }^{14}$ and applied to post mortem tissue ${ }^{15}$, provides good overlap with MASH cleared samples, with resolutions in the range of 10 s of micrometres and field 
of views in the centimetre range. Therefore, the comparison of structural information gained with post mortem UHF MRI, with more specific labels introduced in the cleared samples becomes feasible. In turn, post mortem MRI information can be translated to non-invasive in vivo imaging with the same structural MRI techniques. Studies using such non-invasive methods in animal models, particularly for functional imaging, such as fMRI and fNIRS, would provide an interesting opportunity to deepen our insights into the nature of the blood vessel dependent signals in these functional imaging techniques. These studies would offer the unique opportunity to compare the same specimen after functional imaging with higher resolution vasculature maps obtained with e.g. the angioMASH protocol (Chapter 3). Taken together, the MASH technique in its different variations has the potential to become a standard clearing method in many laboratories, both in the field of neurosciences as well as in other fields.

\section{Clinical translation potential}

Aside from fundamental neuroscientific applications, MASH has already shown its potential to clear FFPE (Formalin-Fixed ParaffinEmbedded) specimen on other tissue types and diseased samples, such as prostatectomy samples (Chapter 5). Hence, it could become relevant for the clinical investigation of pathological specimen, for which the FFPE procedure is a standard preservation protocol. In the field of pathology, the visualisation of whole biopsies or ectomy 
samples would be particularly useful, as it would provide a more sensitive and complete sampling of potential disease marker over the entire extent of the available tissue. In cancer biopsy samples for instance, the categorisation of different cancer stages depends on the visualisation of abnormal cells and structures. Especially at earlier stages, undercounting of these cells is more likely with classical 2D histology which samples only a few out the hundreds or thousands of (potential) slices of an entire biopsy or ectomy. This could lead to a (partial) misclassification and hence in a less suitable treatment plan for patients ${ }^{16}$. 3D histology (or more succinctly: 3D pathology) could therefore help patients receive a more accurate diagnosis and more suitable treatment, through the time- and cost-efficient sampling of entire biopsies. The histochemical dyes neutral red and methylene blue already provided a simple way for a general overview staining on prostate tissue and human endometrium biopsies (the latter processed in a pilot together with the Department for Anatomy and Embryology). For a staining pattern more similar to the traditional Haematoxylin and Eosin (H\&E) staining, the fluorescent properties of $\operatorname{eosin}^{17}$, in combination with methyl green as a marker for DNA could be exploited. One further aspect that has been addressed recently ${ }^{18}$, is the development of faster clearing for a timely diagnosis of biopsy samples. This new dehydration method is potentially compatible with MASH and therefore a promising method for a future pathoMASH pipeline. 
It has also been shown in samples with Alzheimer's disease that the visualisation of disease biomarkers in a $3 \mathrm{D}$ volume can reveal features of the disease that would be very difficult to detect in $2 \mathrm{D}$ slices ${ }^{19}$. For instance, iDISCO+-cleared human hippocampal samples revealed so called Three dimensional Amyloid Patterns (TAPs) forming layered, sheet-like structures in some patients. In other patients, the patterns formed gradients or complex 3D shapes such as ribbons. The high number of amyloid plaques sampled in these volumes also enabled a quantification of the large variability of plaques in terms of their morphology, volume, and inter-plaque distance. The investigation of other brain diseases, such as epilepsy, Parkinson's disease or vascular dementia, with 3D histology could lead to the discovery of similar spatial relations of biomarkers or pathological brain architecture, which have so far been overlooked.

\section{ct-dSPIM}

The ct-dSPIM (cleared-tissue dual view Selective Plane Illumination Microscopy) set-up introduced in Chapter 5 is an oblique light-sheet system optimized for very large cleared samples, which was codeveloped with Applied Scientific Instrumentation (ASI Inc., Eugene, US). Together with the MFS (Mesoscopic Fast Scan) imaging method, mesoscopic overview scans of large regions or entire samples can be acquired at a fast rate and with adjustable resolution without the need to change the optics of the microscope. 


\section{Dissemination and current applications}

The full details of the set-up including all components and the MFS imaging method will be made openly available upon publication. The design of this system has already been presented at SfN and LSFM in 2018 and has been well received within the light-sheet microscopy community. Furthermore, the group in which the work for this thesis has been conducted was recently awarded a VENI grant (awarded to Dr. Anna Schueth) to advance the hardware of the system, to further utilize its potential for neuroscientific and clinical 3D imaging. In this context, the set-up at Maastricht University is embedded at MHeNS and is already used in collaborative projects on prostate cancer imaging, together with the Department of Pathology. Further collaborative projects are already scheduled with both the Cardiovascular Research Institute Maastricht (CARIM) and Division 3 (Translational Neuroscience) of MHeNs at Maastricht University involving whole brain imaging of mouse and rat brains. The large imaging chambers of the ct-dSPIM (allowed by the oblique setup and large translation stage) in principle allow for the rapid highthroughput acquisitions of multiple rodent brains in parallel.

The imaging of more diverse tissue types from biopsy, ectomy, or post mortem samples is already planned together with both, the Department of Anatomy and Embryology and the Department of Pathology. Future adjustments to the ct-dSPIM system could allow for higher resolution imaging than currently possible and hence make it 
an even more attractive imaging solution for other groups at Maastricht University and outside. To achieve this, prospective hardware updates with higher magnification objectives and the installation of a static light-sheet generated by a cylindrical lens, in order to allow for axial scanning at near isotropic resolution without the need for multi view deconvolution, is necessary.

\section{Wider scientific and academic potential}

This system is not only relevant for neuroscience, but potentially for all areas of structural/anatomical investigations of human organs and those of other species, for questions in which a large field of view with relatively low microscopic resolution is required. Therefore, the current system has a wide applicability in many fields of fundamental and clinical research. To further expand the potential range of applications to even more diverse fields and research questions, the effective resolution of the system could be increased not only by the hardware updates indicated above, but also in combination with new histological methods such as expansion microscopy (ExM) ${ }^{20,21}$. ExM effectively increases the resolution, by physically separating structures of interest while maintaining their original spatial relationship ${ }^{22-25}$. This would allow for the investigation of structures which are impossible to resolve on the current system. 


\section{Clinical translation potential}

As mentioned above, the set-up at Maastricht University is already used in a collaborative project on prostate cancer imaging, together with the Department of Pathology. In this project, the potential of the ct-dSPIM in combination with MASH optimised for FFPE tissue is explored for the fast 3D visualisation and classification of these prostate cancer samples. The large imaging chamber of the ct-dSPIM allows for the parallel mounting and rapid imaging of many clinical specimen in parallel, which makes the ct-dSPIM particularly interesting for diagnostic high-throughput imaging. Instead of slicing multiple thin sections out of e.g. a core needle prostate biopsy, many such biopsies from the same patient or from several patients could be quickly imaged together in a single acquisition at a mesoscopic resolution. Diagnosis could either be performed on site during live imaging by the pathologist or at a later point after inspection of the $3 \mathrm{D}$ rendered dataset. In the latter case, the imaging would be decoupled from the diagnosis and, therefore, the microscopist would be able to focus exclusively on data acquisition, further increasing the throughput speed of the pipeline. This potential for massive parallelizing of the imaging could potentially outweigh the comparatively longer processing times of the current pipeline as compared to standard thin sections stained with the H\&E staining (however see comment above on potential decrease in processing time in the MASH section). It should also be considered that processing entire biopsies into hundreds to thousands of classical 
sections and the inspection of these would take much longer than MASH processing followed by 3D inspection. It is assumed, however, that while the $3 \mathrm{D}$ imaging of whole clinical specimens is ultimately superior to standard 2D histology, it will take some time for this method to become an accepted, let alone a standard tool in diagnostics. 3D histological pipelines need to be made highly robust and reliable to work in a variety of sample types while providing a similar visual appearance as H\&E stained sections, as discussed above in the MASH section. The coordination of the image acquisition and the diagnostic inspection of acquired data is another step in the pipeline which needs to become more standardized. As the widespread implementation of light-sheet systems on the pathologist's desk is considered unlikely, better ways to quickly share data or live stream acquisitions would be highly useful. Despite these significant current limitations, the long-term clinical impact of fast volumetric microscopy methods is assumed to be considerable and its routine implementation on pathology labs ultimately only a question of time.

\section{hFRUIT}

Chapter 6 presents a clearing approach fundamentally different from MASH. hFRUIT is an adapted version of the aqueous clearing protocol FRUIT, optimized for human brain tissue. Both the original FRUIT and hFRUIT are simple to perform and do not require any specialised equipment. Since hFRUIT does not remove the brain's lipids, it is 
uniquely suited for the combination with lipophilic dyes to trace intrinsic cortical connections in human brain tissue. This however, comes with the trade-off of a reduced clearing capacity.

\section{Dissemination and current applications}

The hFRUIT protocol is available as an open access publication ${ }^{26}$ and was presented at both the SfN and LSFM conferences in 2016. The hFRUT protocol is has an important niche application, as the injection of lipophilic dye crystals is the only method to study the interareal connectivity in the human brain by directly visualising specific axonal connections down to the single fibres and retrogradely labelled neuron populations. With hFRUIT it is now possible to view these labelled connections directly in $3 \mathrm{D}$, at a microscopic resolution and yet over mesoscopic distances (tracer diffusion is limited to about 1-2 $\mathrm{cm})$. Given the highly specialised character of this clearing protocol, the future application of this method is seen in fundamental neuroscientific research, with potential for further academic rather than clinical or commercial impact. For instance, the application of hFRUIT to existing as well as future material from the collection of the Systems Neurophysiology group of the Technische Universität Darmstadt, with injection sites in different brain areas, is planned in connection with our ongoing collaboration. 


\section{Wider scientific and academic potential}

The virtual dissection of cortical microcircuitry, in combination with the cytoarchitecture stainings developed in the MASH protocol, will hopefully broaden our understanding of the minute differences in that circuitry between different brain areas. The combination of labels for cell bodies used in MASH could provide further anatomical information regarding the exact location of intrinsic connections by revealing the layered cortical architecture. The unique compatibility of lipophilic dyes with 3D imaging makes aqueous non-delipidating clearing protocols such as hFRUIT highly interesting tools in the field of human brain connectomics. Aside from the knowledge on intrinsic connectivity gained directly by this method, it could be used in combination with post mortem diffusion-weighted MRI (dMRI) to validate the latter method ${ }^{27}$. The combination of lipophilic tracers with dMRI has been used by the lab hosting this thesis work in the past ${ }^{28}$, however the histological analysis in that dMRI validation work was limited on 2D sections. With hFRUIT, these validation experiments could now be performed in 3D to further optimize e.g. dMRI tractography and connectomics methods ${ }^{29,30}$ in order to derive a more precise representation of human brain connectivity. Additionally, the discovery of the positive effect of the PLP (periodate-lysineparaformaldehyde) fixation ${ }^{31}$ on the clearing capacity is of interest to the wider clearing community. The tissue fixation itself has not received much attention as a factor influencing the performance of clearing protocols and different fixation methods could further 
improve other clearing methods as well. It could therefore be possible to produce even more highly transparent samples and enabling even deeper imaging with less light scattering, when combining more potent clearing methods such as MASH with these beneficial fixation methods.

\section{Conclusion}

Taken together, the work presented in this thesis introduces a set of techniques and devices to bring human tissue clearing to a different scale as well as to more diverse fields of research. While this thesis does not address every aspect necessary for the 3D histological investigation of human brain tissue, it demonstrates substantial progress on the tissue processing and imaging aspects of such a pipeline. Scalable labelling strategies for brain angio- and cytoarchitecture have been developed together with a clearing pipeline that can process large parts of the human brain, or other organs such as the prostate. The further upscaling of these methods to whole human brain slices of up to $5 \mathrm{~mm}$ thickness is feasible and the microscope platform presented in this thesis would allow for the fast mesoscopic imaging of samples this size. A more specialised clearing protocol for the investigation of intrinsic human connectivity could greatly improve our insights into cortical circuitry and its variations between areas. The techniques presented in this thesis where primarily designed for their use in fundamental neuroscience. However, they are potentially interesting for many academic 
disciplines including clinical fields, such as pathology or clinical neuroscience, and the ct-dSPIM developments were performed in collaboration with a commercial company, showing further potential for commercial valorisation. Therefore, the developments reported in this thesis, have the long-term potential to benefit not only the fundamental understanding of the human brain, but also to improve diagnostics and hence eventually benefit clinicians and patients. 


\section{References}

1 Hildebrand, S., Schueth, A., Herrler, A., Galuske, R. \& Roebroeck, A. Scalable Labeling for Cytoarchitectonic Characterization of Large Optically Cleared Human Neocortex Samples. Scientific reports 9, 10880, doi:10.1038/s41598-019-47336-9 (2019).

2 Voigt, F. F. et al. The mesoSPIM initiative: open-source light-sheet microscopes for imaging cleared tissue. Nature methods 16, 1105-1108, doi:10.1038/s41592-019-0554-0 (2019).

3 Chung, K. et al. Structural and molecular interrogation of intact biological systems. Nature 497, 332-337, doi:10.1038/nature12107 (2013).

4 Dodt, H. U. et al. Ultramicroscopy: three-dimensional visualization of neuronal networks in the whole mouse brain. Nature methods 4, 331336, doi:10.1038/nmeth1036 (2007).

5 Renier, N. et al. iDISCO: a simple, rapid method to immunolabel large tissue samples for volume imaging. Cell 159, 896-910, doi:10.1016/j.cell.2014.10.010 (2014).

6 Zheng, H. \& Rinaman, L. Simplified CLARITY for visualizing immunofluorescence labeling in the developing rat brain. Brain Structure and Function 221, 2375-2383, doi:10.1007/s00429-015-10200 (2016).

7 Branch, A., Tward, D., Vogelstein, J. T., Wu, Z. \& Gallagher, M. An optimized protocol for iDISCO+ rat brain clearing, imaging, and analysis. bioRxiv, 639674, doi:10.1101/639674 (2019).

8 Tainaka, K. et al. Chemical Landscape for Tissue Clearing Based on Hydrophilic Reagents. Cell reports 24, 2196-2210 e2199, doi:10.1016/j.celrep.2018.07.056 (2018). 
9 Economo, M. N. et al. Distinct descending motor cortex pathways and their roles in movement. Nature 563, 79-84, doi:10.1038/s41586-0180642-9 (2018).

10 Economo, M. N., Winnubst, J., Bas, E., Ferreira, T. A. \& Chandrashekar, J. Single-neuron axonal reconstruction: The search for a wiring diagram of the brain. The Journal of comparative neurology 527, 2190-2199, doi:10.1002/cne.24674 (2019).

11 Morawski, M. et al. Developing 3D microscopy with CLARITY on human brain tissue: Towards a tool for informing and validating MRI-based histology. Neuroimage 182, 417-428, doi:10.1016/j.neuroimage.2017.11.060 (2018).

12 Jobsis, F. F. Noninvasive, infrared monitoring of cerebral and myocardial oxygen sufficiency and circulatory parameters. Science 198, 1264, doi:10.1126/science.929199 (1977).

13 Kandice, T. et al. Spectrally resolved neurophotonics: a case report of hemodynamics and vascular components in the mammalian brain. Journal of biomedical optics 10, 1-9, doi:10.1117/1.2137291 (2005).

14 Dumoulin, S. O., Fracasso, A., van der Zwaag, W., Siero, J. C. W. \& Petridou, N. Ultra-high field MRI: Advancing systems neuroscience towards mesoscopic human brain function. Neurolmage 168, 345-357, doi:https://doi.org/10.1016/j.neuroimage.2017.01.028 (2018).

15 Sengupta, S. et al. High resolution anatomical and quantitative MRI of the entire human occipital lobe ex vivo at 9.4T. Neurolmage 168, 162171, doi:https://doi.org/10.1016/j.neuroimage.2017.03.039 (2018).

16 Glaser, A. K. et al. Light-sheet microscopy for slide-free non-destructive pathology of large clinical specimens. Nature biomedical engineering 1, doi:10.1038/s41551-017-0084 (2017).

17 Serafin, R., Xie, W., Glaser, A. K. \& Liu, J. T. C. FalseColor-Python: A rapid intensity-leveling and digital-staining package for fluorescence-based slide-free digital pathology. Plos one 15, e0233198, doi:10.1371/journal.pone.0233198 (2020). 
18 Sabdyusheva Litschauer, I. et al. 3D histopathology of human tumours by fast clearing and ultramicroscopy. Scientific reports 10, 17619, doi:10.1038/s41598-020-71737-w (2020).

19 Liebmann, T. et al. Three-Dimensional Study of Alzheimer's Disease Hallmarks Using the iDISCO Clearing Method. Cell reports 16, 11381152, doi:10.1016/j.celrep.2016.06.060 (2016).

20 Karagiannis, E. D. \& Boyden, E. S. Expansion microscopy: development and neuroscience applications. Current opinion in neurobiology 50, 5663, doi:10.1016/j.conb.2017.12.012 (2018).

21 Wassie, A. T., Zhao, Y. \& Boyden, E. S. Expansion microscopy: principles and uses in biological research. Nature methods 16, 33-41, doi:10.1038/s41592-018-0219-4 (2019).

22 Gao, R. et al. Cortical column and whole-brain imaging with molecular contrast and nanoscale resolution. Science 363 , doi:10.1126/science.aau8302 (2019).

$23 \mathrm{Ku}, \mathrm{T}$. et al. Multiplexed and scalable super-resolution imaging of threedimensional protein localization in size-adjustable tissues. Nature biotechnology 34, 973-981, doi:10.1038/nbt.3641 (2016).

24 Murakami, T. C. et al. A three-dimensional single-cell-resolution wholebrain atlas using CUBIC-X expansion microscopy and tissue clearing. Nature neuroscience 21, 625-637, doi:10.1038/s41593-018-0109-1 (2018).

25 Tillberg, P. W. et al. Protein-retention expansion microscopy of cells and tissues labeled using standard fluorescent proteins and antibodies. Nature biotechnology 34, 987-992, doi:10.1038/nbt.3625 (2016).

26 Hildebrand, S. et al. hFRUIT: An optimized agent for optical clearing of Dil-stained adult human brain tissue. Scientific reports 10, 9950, doi:10.1038/s41598-020-66999-3 (2020). 
27 Roebroeck, A., Miller, K. L. \& Aggarwal, M. Ex vivo diffusion MRI of the human brain: Technical challenges and recent advances. NMR in Biomedicine 32, e3941, doi:https://doi.org/10.1002/nbm.3941 (2019).

28 Seehaus, A. K. et al. Histological validation of DW-MRI tractography in human postmortem tissue. Cerebral cortex 23, 442-450, doi:10.1093/cercor/bhs036 (2013).

29 Jeurissen, B., Descoteaux, M., Mori, S. \& Leemans, A. Diffusion MRI fiber tractography of the brain. NMR in Biomedicine 32, e3785, doi:https://doi.org/10.1002/nbm.3785 (2019).

30 Sotiropoulos, S. N. \& Zalesky, A. Building connectomes using diffusion MRI: why, how and but. NMR in Biomedicine 32, e3752, doi:https://doi.org/10.1002/nbm.3752 (2019).

31 McLean, I. W. \& Nakane, P. K. PERIODATE-LYSINE-PARAFORMALDEHYDE FIXATIVE A NEW FIXATIVE FOR IMMUNOELECTRON MICROSCOPY. Journal of Histochemistry \& Cytochemistry 22, 1077-1083, doi:10.1177/22.12.1077 (1974). 


\section{Abbreviations}

$\begin{array}{ll}\text { A } & \text { Anterior } \\ \text { AF } & \text { Autofluorescence } \\ \text { AO } & \text { Acridine orange } \\ \text { BA } & \text { Benzyl alcohol } \\ \text { BB } & \text { Benzyl benzoate } \\ \text { BF } & \text { brightfield } \\ \text { CA } & \text { Cinnamaldehyde } \\ \text { cm } & \text { Centimetre } \\ \text { CS } & \text { Calcarine sulcus } \\ \text { ct-dSPIM } & \text { cleared tissue dual-view Selective Illumination Microscopy } \\ \text { CV } & \text { Cresyl violet acetate } \\ \text { d } & \text { Dorsal } \\ \text { DAPI } & \text { 4',6-Diamidino-2-phenylindol } \\ \text { DBE } & \text { Dibenzyl ether } \\ \text { DCM } & \text { Dichloromethane } \\ \text { DiD } & \text { DilC18(3) (1,1'-Dioctadecyl-3,3,3',3'-Tetrameth } \\ & \text { ylindodicarbocyanine Perchlorate) } \\ \text { Dil } & \text { DilC18(5) (1,1'-Dioctadecyl-3,3,3',3'- } \\ \text { DiR } & \text { Tetramethylindocarbocyanine Perchlorate) } \\ \text { ECi } & \text { DilC18(7) (1,1"-dioctadecyl-3,3, 3",3"- } \\ \text { EFL } & \text { Tetramethylindotricarbocyanine lodide) } \\ \text { EtOH } & \text { Effective focal length } \\ \text { FDM } & \text { Ethanol } \\ \text { FFF } & \text { Fused deposition modeling } \\ \text { Fig. } & \text { Fused Filament Fabrication } \\ \text { FOV } & \text { Figure } \\ \text { fMRI } & \text { Field of view } \\ \text { gm } & \text { functional Magnetic Resonance Imaging } \\ \text { hFRUIT } & \text { Grey matter } \\ & \text { human FRUIT } \\ & \\ & \end{array}$




$\begin{array}{ll}\text { iDISCO } & \begin{array}{l}\text { immunolabeling-enabled three-dimensional imaging of solvent- } \\ \text { cleared organs }\end{array} \\ \text { L } & \text { Lateral } \\ \text { LEL } & \text { Lycopersicon esculentum lectin } \\ \text { LSFM } & \text { Light-sheet fluorescent microscopy } \\ \text { MASH } & \text { Multiscale Architectonic Staining of Human cortex } \\ \text { mm } & \text { Millimetre } \\ \text { mM } & \text { Millimolar } \\ \text { mW } & \text { Milliwatt } \\ \text { M } & \text { medial } \\ \text { MB } & \text { Methylene blue } \\ \text { MeOH } & \text { Methanol } \\ \text { MG } & \text { Methyl green } \\ \text { MRI } & \text { Magnetic Resonance Imaging } \\ \text { Na } & \text { Numerical apperture } \\ \text { NR } & \text { Neutral red } \\ \text { nm } & \text { Nanometer } \\ \text { OCT } & \text { Optical Coherence Tomography } \\ \text { OP } & \text { Occipital pole } \\ \text { P } & \text { Posterior } \\ \text { PBS } & \text { Phosphate buffered saline } \\ \text { PBST } & \text { Phosphate buffered saline }+ \text { O.2\% Triton X-100 } \\ \text { PLP } & \text { Periodate-lysine-paraformaldehyde fixative } \\ \text { PP } & \text { Polypropylene } \\ \text { PTFE } & \text { Polytetrafluoroethylene } \\ \text { ROI } & \text { Region of Interest } \\ \text { SCC } & \text { Splenium of the corpus callosum } \\ \text { SDS } & \text { Sodium dodecyl sulfate } \\ \text { SLA } & \text { Steroelithography } \\ \text { SLS } & \text { Selective Laser Sintering } \\ \text { SPIM } & \text { Selective Plane Illumination Microscopy } \\ \text { SWITCH } & \text { System-Wide control of Interaction Time and kinetics } \\ \text { suppl. } & \text { of CHemicals } \\ \text { TBS } & \text { Tripplemental information } \\ & \end{array}$




$\begin{array}{ll}\text { TDE } & \text { 2,2'-thiodiethanol } \\ \text { TPLSM } & \text { Two-photon laser scanning microscopy } \\ \text { UHF } & \text { ultra-high field } \\ v & \text { Ventral } \\ \text { V1 } & \text { primary Visual cortex } \\ \text { V2 } & \text { secondary Visual cortex } \\ \text { WD } & \text { Working distance } \\ \text { WGO } & \text { wintergreen oil } \\ \text { wm } & \text { White matter } \\ \mu l & \text { Microliter } \\ \mu m & \text { Micrometer } \\ \text { 3D } & \text { Three-dimensional } \\ \text { 2D } & \text { Two-dimensional }\end{array}$




\section{Acknowledgements}

"Well that took longer than expected", but even this thesis is coming to an end. Although it does not always feel like it right at the time, this $\mathrm{PhD}$ was very much a team effort and would not have been possible without almost constant help by one person or the other. So of course, these people will have to go through the ordeal of being called out and read how incredibly important and helpful they are. It's a drag I know, so brace for it:

The first person, I would like to thank is Alard, my first promotor and supervisor. Alard, you were always so engaged and yet patient in our discussions and meetings and always open for any crazy and weird idea I had. Whether it was something actually related to the topic or one of my many, many random interludes on the recent 3D printed gimmick, you were always enthusiastic about it. This helped me a lot to settle into the $\mathrm{PhD}$ role, especially in the beginning, when I was still very germanized (or rather even more so than now) and thought every casual hallways "how are things going" question is a prompt to report the recent lab progress. I still occasionally do it, but I think I am getting better... or at least more random. Thank you also for giving me so many opportunities to simply try things out in the lab, even though so many of them did not work in the end. I really enjoyed working on this project (most of the time, it was still a PhD after all) and all the nonwork-related talks with the group. Although I am one of the freak 
people who finds bikes rather dull pieces of hardware... sorry about that... and SciFi is still inferior to fantasy I'm afraid. But you got me to read the neuromancer books and they are rather ok I guess...

The next person in line whom I want to thank is Ralf. Without you, I would have never come to Maastricht in the first place. So thank you for enabling that connection and thereby making all of this possible. But you did more than that. It was in your lectures that I first got into contact with neuroanatomy and in which I got fascinated by the topic. And it was in your group where I did my first histological stainings as well as my first steps into tissue clearing. So for better or worse, you kind of dragged me into this and I fear there is no escape for me now. I also really enjoyed any opportunity to talk with you about neuroscience. Your expertise and experience in the field is very valuable to me and gave me new lines of thought so many times. I still remember when in one of our lab meetings I was musing with Miriam about a staining for both myelinated fibres and Nissl on the same sections and you remarking off-handedly "Oh das wäre dann die Klüver-Barrera-Färbung.", immediately destroying my hopes of just having thought a totally new and innovative staining idea. Well I guess you can't always have everything...

Rainer, of course this PhD would ultimately not have been possible without you as well, so thank you for that. Although we have not met very often in my time as a $\mathrm{PhD}$ student, on the occasion when we did, your enthusiasm for my project and all the long-term possibilities of 
it was always inspiring and it provided me with a more broad- and long-term perspective of things, so thank you especially for this as well.

And of course I want to thank the CBClab in general: I had a great time with all of you guys and cannot say enough how often and how much you have helped me out over the years.

Anna, you helped me a lot in the beginning of my $\mathrm{PhD}$ with getting used to conferences and were often my first address when I had any question related to academia. And of course without you being locked into a tiny dark room for long stretches of time, I would have much less cool data to show off with.

Arko, you were a central part of our group and I cannot begin to express how cool it was to have an engineer in the same room, whom I could always pester when I had any vague idea about hardware. Even more important, you were able to derive some meaning out of my toddler-level sketches that usually went along with those ideas and actually design something real out of it. Amazing... Last but not least you agreed to be my paranymph, which I am aware is quite the pain in the ass, so thank you big time for that as well.

Sri I know you are not a CBClab member, but I consider you an extended member of the lab. That's how it is now, no way out of it anymore. Your critical view on things really helped me when discussing science or basically any other topic. And of course, you and Arko went through the ordeal of proofreading my thesis for which I am VERY grateful. It was also always fascinating to hear both of you 
talk about current developments in India. This definitely widened my perspective on a lot of things and humbled me by showing in yet another way just how many things I do not know. Well, it did all of that when you were talking in a language with each other that I could actually understand.

Johannes, you should just drop this whole science stuff and just become a baker. Seriously, your cookies and bread are so good... I wonder if there is anything you cannot do. After any of our neurociency chats, I always have something new to think about. I really appreciate how you give me a completely different perspective on things. And of course you agreed to be my paranymph pretty much last minute for which I am very VERY grateful.

The same goes for you Michael. Your views on the topic are so different from mine that it sometimes is challenging for me to even get what you are talking about, but it is (almost) always worth to take the time and get your point of views. You also triggered me into enjoying teaching much more and I think those courses with you were always great fun... Although the students clearly did not like my exam questions about mouse mutants and fish with weird eyes on top of their head. However, even more important was your support in the last few months. Without you, this thesis definitely would not have been finished in time and your support to me and the whole group was invaluable. Thank you for that.

Of course, I also miss those evenings together with all of us at Robbert's or Arko's place. It was always great fun, although my 
memories about a few of those evenings are somewhat hazy for some weird reason.

I also want to thank Hellen Steinbusch and the technician team of the neuroscience department. Without all of you, this thesis definitely would not have happened. Thank you so much for all your support and help and the unnerving ability to always materialize chemicals out of nowhere and on short notice when needed. In the beginning of the corona pandemic, when working was very difficult for everyone, you even managed to provide me with my own private lab and boy how much I miss having that luxury!

Obviously there were many colleagues, at FPN and FHML and from my old group in Darmstadt, who helped me out in numerous ever so small and yet significant ways. I won't go through the trouble of naming you all, because then I will forgot and unintentionally insult someone while being blissfully unaware of it. So just imagine your name right here:

. I know I'm not the most social type and don't mingle that often, but the little chats either complaining about work or talking about completely unrelated things really helped me to keep my sanity even in the most stressful times (well at least I think it did, but that probably has to be judged by others).

To reduce my credit for this thesis yet a bit more, I want to thank all the smart and diligent student who helped me out over the years. You guys definitely were a great help with my experiments and I could not have done nearly as many of my crazy staining experiments without 
you. But some of you also became personal friends over time, so I want to thank specifically Annemarie, Jana and Jasmine for being such great company, not only in the lab but also after work. I really enjoyed our coffee (or cocktail) talks and will miss those a lot. And to our new students: Hanna, Jinthe and Maite, thanks for picking up the debris I left and driving the work further on. Ganbare!

Last but not least, I want to thank my family for their unwavering (financial) support over the years. I can imagine it must have been very frustrating sometimes, but see how all the time and work culminated in a very large and pretty piece of paper. 


\section{Curriculum Vitae}

Sven Hildebrand was born on Sunday the $25^{\text {th }}$ of February 1990 in Zeitz, Germany where he spent the first 8 years of his life. In his $3^{\text {rd }}$ elementary school year, he moved into the no man's land of the northwestern-most Franconian vestiges, politically and mentally Bavarian, but culturally and dialectically Hessian. It was here that he was first culture-shocked without even leaving his home country and where he obtained his first memories of confrontation with religious beliefs, which would be a continuous theme in his later life. Sven spent most of his formative years close to the city of Aschaffenburg, where he also frequented the Friedrich-Dessauer-Gymnasium, although especially in his later years in this school he did not frequent it as much as the schedule actually demanded. Nevertheless, he finished his Abitur in 2009. At this time it was already clear for him that he wanted to pursue a study program in biology, even though at that time his vision of a biologist was not very representative. For his bachelor studies in biology, he went back to his birth state of Sachsen-Anhalt, to the Martin-Luther-Universität Halle-Wittenberg. Here, he specialised in plant and animal physiology in his advanced classes. The latter course formed the basis of his bachelor thesis in the possible pheromonedriven origins of the aggregated egg-laying behaviour of the desert locust Schistocerca gregaria, under the supervision of Dr. Karsten Seidelmann and Prof. Dr. Hans-Jürgen Ferenz. This work initiated his interest in animal behaviour and its causes, and hence in 
neurobiology. He therefore enrolled in the Technical Biology program at the Technische Universität Darmstadt, where he gained more insights into neuroanatomy and histology. Not only were these two disciplines at the core of his master thesis on morphological changes in the brains of glycine-receptor-deficient mice, but the start of an ongoing interest. To further deepen his knowledge in these areas, he followed up on his thesis by working as a research assistant in the same group, in which he conducted his master thesis experiments, the group of Prof. Dr. Ralf Galuske. It was during this time that he came into first contact with the emerging techniques of tissue clearing and light-sheet microscopy when collaborating with Dr. Francesco Pampaloni, in the group of Prof. Dr. Ernst Stelzer at the GoetheUniversität in Frankfurt am Main, and he was immediately thrilled by them. For this reason he considered himself very lucky, when he was able to follow up on his work as a research assistant, with a $\mathrm{PhD}$ project in the group of Dr. Alard Roebroeck. He devoted the next few years to the development of optical clearing and labelling methods for human brain tissue and the imaging thereof. He also tried to further deepen his knowledge of neuroanatomy by acquiring as many books on the subject as he could, hoping that by surrounding himself with them, the knowledge would somehow seep through osmosis into his brain, although he also read a few of them. Following the completion of his $\mathrm{PhD}$, Sven hopes to be able to follow his passion in the investigation of human and non-human neuroanatomy with the newly emerging toolkit of 3D histology methods. 


\section{List of Publications}

\section{Published journal articles}

Hildebrand, S, Schueth, A, Wangenheim, K v, Mattheyer C, Pampaloni F, Bratzke H, Roebroeck A, Galuske R. hFRUIT: An optimized agent for optical clearing of DiI-stained adult human brain tissue. Scientific Reports 10, 9950 (2020). doi:10.1038/s41598-020-66999-3

Voigt F., Kirschenbaum D, Platonova E, Pagès S, Campbell R, Kästli R, Schaettin M, Egolf L, van der Bourg A, Bethge P, Haenraets K, Frézel N, Topilko T, Perin P, Hillier D, Hildebrand S, Schueth A, Roebroeck A, Roska B, Stoeckli E, Pizzala R, Renier N, Zeilhofer H, Karayannis T, Ziegler U, Batti L, Holtmaat A, Lüscher C, Aguzzi A, Helmchen F. The mesoSPIM initiative: opensource light-sheet mesoscopes for imaging in cleared tissue. Nature Methods 16, 1105-1108 (2019). doi:10.1038/s41592-019-0554-0

Hildebrand S, Schueth A, Herrler A, Galuske R, Roebroeck A. Scalable Labeling for Cytoarchitectonic Characterisation of Large Optically Cleared Human Neocortex Samples. Scientific Reports 9, 10880 (2019). doi:10.1038/s41598019-47336-9

Sengupta S, Fritz F J, Harms R L, Hildebrand S, Tse D H Y, Poser B A, Goebel R, Roebroeck A. High resolution anatomical and quantitative MRI of the entire human occipital lobe ex vivo at 9.4T. NeuroImage 168, 62-171 (2018). https://doi.org/10.1016/j.neuroimage.2017.03.039. 


\section{Journal articles in preparation}

Hildebrand S, Bethghe P, Capalbo M, Herrler A, Helmchen F, Roebroeck A. An investigation of angioarchitecture in the human visual cortex with angioMASH tissue clearing and labelling. In prep.

Schueth A, Hildebrand S, Samarska I, Sengupta S, Kiessling A, Herrler A, zur Hausen A, Roebroeck A. cleared tissue dual Selective Plane Illumination Microscopy (ct-dSPIM) for fast mesoscopic imaging of large-scale human tissues. In prep.

Hildebrand S, Sengupta S, Schueth S, Herrler A, Roebroeck A. Taking MASH to the next level: A cost-effective high-throughput pipeline for the investigation of cytoarchitecture in large human brain slices. In prep.

\section{Conference oral presentations}

Hildebrand S, Bethge P,Voigt F F, Helmchen F, Roebroeck A. Characterization of neocortical angioarchitecture across layers and areas in the human brain. Virtual $12^{\text {th }}$ Light Sheet Fluorescence Microscopy (LSFM) conference (2020) doi:10.22443/rms.lsfm2020.38

Schueth A, Hildebrand S, Roebroeck A. A platform for two-photon and lightsheet microscopic imaging of optically cleared and fluorescently labelled human brain tissue. Light Sheet Fluorescence Microscopy (LSFM) conference, Singapore (2017) 


\section{Conference poster presentation}

Hildebrand S, Schueth A, Sengupta S, Kiessling A, Herrler A, Galuske R, Roebroeck A. A scalable processing pipeline for high-throughput staining of cortical architecture in large archival human brain samples. Swiss Light-sheet Microscopy Workshop, Zurich, Swizerland (2019)

Hildebrand S, Schueth A, Sengupta S, Kiessling A, Herrler A, Galuske R, Roebroeck A. A scalable processing pipeline for high-throughput optical clearing and labelling of very large formalin-fixed human brain samples. Society for Neuroscience (SfN) conference, San Diego, US (2018)

Hildebrand S, Schueth A, Kiessling A, Herrler A, Galuske R, Roebroeck A.

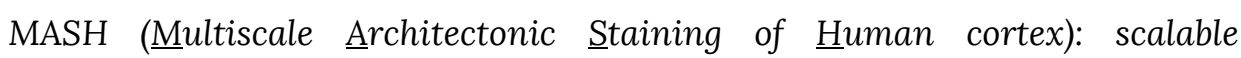
cytoarchitectonic characterization of large optically cleared human neocortex samples in 3D. Light Sheet Fluorescence Microscopy (LSFM) conference, Dresden, Germany (2018)

Hildebrand S, Schueth A, Roebroeck A. MASH (ㅆ ultiscale Architectonic Staining of Human cortex) enables optical clearing and labelling of large adult human brain samples for 3D cytoarchitecture. Society for Neuroscience (SfN) conference, Washington D. C., US (2017)

Hildebrand S, Schueth A, Roebroeck A. Clearing and cytoarchitecture labelling techniques compatible with large human cortex samples. Light Sheet Fluorescence Microscopy (LSFM) conference, Singapore (2017)

Hildebrand S, Schueth A, von Wangenheim K, Roebroeck A, Galuske R. DiIcompatible, immersion-based clearing of brain tissue: Comparison of different 
clearing and fixation protocols. Society for Neuroscience (SfN) conference, San Diego, US (2016)

Hildebrand S, von Wangenheim K, Roebroeck A, Galuske R. Comparison of fructose/urea immersion-based protocols for optical clearing of brain tissue. Light Sheet Fluorescence Microscopy (LSFM) conference, Sheffield, UK (2016) 